\title{
Regio- and Stereoselective Synthesis of Multi-Alkylated Allylic Boronates through Three-Component Coupling Reactions between Allenes, Alkyl Halides and a Diboron Reagent
}

\section{Yu Ozawa, ${ }^{\dagger}$ Kohei Endo, ${ }^{\dagger}$ Hajime Ito $^{\dagger+*}$}

†Division of Applied Chemistry, Graduate School of Engineering, Hokkaido University, Sapporo, Hokkaido 060-8628, Japan

Institute for Chemical Reaction Design and Discovery (WPI-ICReDD), Hokkaido University, Sapporo, Hokkaido 060-8628, Japan

e-mail: hajito@eng.hokudai.ac.jp

\section{Table of Contents}

1. Instrumentation and Chemicals

2. Substrate Preparation Procedure

3. Substrate Characterization

4. General Borylation Procedure

5. Borylation Product Characterization

6. Large Scale Reaction and Synthetic Applications of Borylation Products

7. Procedure of Allylboration Reactions

8. Evaluation of Accuracy of ${ }^{13} \mathrm{C}$ NMR Analysis for Stereoselectivity Determination

9. 2D NMR Study for Determination of Product Stereoselectivity

11. Stoichiometric Reaction

12. Single Crystal X-ray Structural Analysis

13. Computational Study 


\section{Instrumentation and Chemicals}

Materials were obtained from commercial suppliers and purified by standard procedures unless otherwise noted. Solvents were also purchased from commercial suppliers, degassed via three freezepump-thaw cycles, and further dried over molecular sieves (MS 4A). NMR spectra were recorded on JEOL JNM-ECX400P, JNM-ECS400, and JNM-ECA600 spectrometers $\left({ }^{1} \mathrm{H}: 400\right.$ or $600 \mathrm{MHz},{ }^{13} \mathrm{C}$ : $\left.100 \mathrm{MHz}{ }^{11} \mathrm{~B}: 126 \mathrm{MHz}\right)$. Tetramethylsilane $\left({ }^{1} \mathrm{H}, \delta 0.00\right), \mathrm{CDCl}_{3}\left({ }^{13} \mathrm{C}, \delta 77.0\right)$ and $\mathrm{BF}_{3} \cdot \mathrm{OEt}_{2}\left({ }^{11} \mathrm{~B}, \delta\right.$ $0.00)$ were employed as the external standards, respectively. $\mathrm{CuCl}(224332-25 \mathrm{G}, \geq 99 \%)$ and $\mathrm{K}(\mathrm{O}-t-$ $\mathrm{Bu})(659878-5 \mathrm{G}, 99.99 \%)$ were purchased from Sigma-Aldrich Co. and used as received. GLC analyses were conducted with a Shimadzu GC-2014 or GC-2025 equipped with a ULBON HR-1 glass capillary column (Shinwa Chemical Industries) and an FID detector. Recycle preparative gel chromatography (GPC) was conducted with JAILC-9101 using $\mathrm{CHCl}_{3}$ as an eluent. High-resolution mass spectra were recorded at the Global Facility Center, Hokkaido University and GC-MS \& NMR Lab., Faculty of Agriculture, Hokkaido University. Single crystal X-ray structural analysis was carried out on a Rigaku XtaLAB PRO MM007 diffractometer using graphite monochromated Mo- $\mathrm{K}_{a}$ and $\mathrm{Cu}$ $\mathrm{K}_{\alpha}$ radiation. The structure was solved by direct methods and expanded using Fourier techniques. Nonhydrogen atoms were refined anisotropically. Hydrogen atoms were refined using the riding model. All calculations were performed using the Olex2 crystallographic software package except for refinement, which was performed using SHELXL. ${ }^{1}$ 


\section{Substrate Preparation Procedure}

All 1,1-di-substituted allenes $(\mathbf{1} \mathbf{a}-\mathbf{1} \mathbf{m})$ and an alkyl iodide $\mathbf{2 h}$ were prepared according to the references and characterized as described below. Other alkyl iodides $(\mathbf{2 a}-\mathbf{2 g}$ and $\mathbf{2} \mathbf{i}-\mathbf{2 n})$ were purchased from commercial suppliers (Tokyo Chemical Industry Co. and Sigma-Aldrich Co.). Spectroscopic data of already known compounds $\left(1 \mathrm{a},{ }^{2} 1 \mathrm{~b},{ }^{3} 1 \mathrm{~g},{ }^{4} 1 \mathrm{l},{ }^{5} 1 \mathrm{~m},{ }^{4}\right.$ and $\left.2 \mathrm{~h}^{6}\right)$ were matched with those reported.

\subsection{General Procedure of gem-disubstituted allenes synthesis (GP1). ${ }^{7}$}

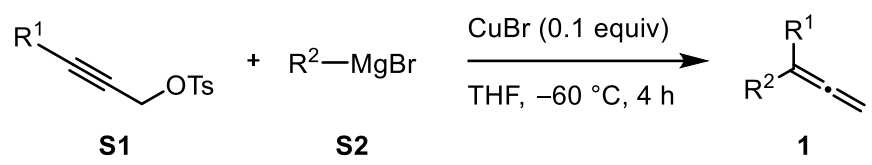

In a vacuum-dried, round-bottomed flask, $\mathrm{CuBr}$ ( 0.1 equiv) and the corresponding propargyl tosylate $\mathbf{S 1}$ (1.0 equiv) were dissolved in dry THF $(0.5 \mathrm{M})$. After the solution was cooled to $-60{ }^{\circ} \mathrm{C}$, the corresponding Grignard reagent $\mathbf{S 2}$ (1.1 equiv) was added dropwise over $1 \mathrm{~h}$ under a nitrogen atmosphere. After stirred for $4 \mathrm{~h}$ at the same temperature, the reaction mixture was warmed up to $0{ }^{\circ} \mathrm{C}$. Then, it was quenched by saturated $\mathrm{NH}_{4} \mathrm{Cl}$ aqueous solution and extracted with $\mathrm{Et}_{2} \mathrm{O}$ three times. The combined organic layer was then dried over $\mathrm{MgSO}_{4}$. After filtration, the solvent was removed by evaporation. The crude mixture was purified by silica gel column chromatography (typically, $\mathrm{Et}_{2} \mathrm{O} /$ hexane $\left.0: 100-5: 95\right)$ to obtain the corresponding 1,1-di-substituted allene product $\mathbf{1}$.

\subsection{General Procedure of gem-disubstituted allenes synthesis (GP2). ${ }^{4}$}

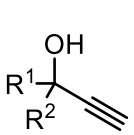

S3

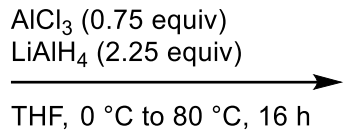

THF, $0{ }^{\circ} \mathrm{C}$ to $80^{\circ} \mathrm{C}, 16 \mathrm{~h}$

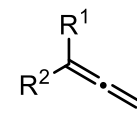

1

In a vacuum-dried, round-bottomed flask equipped with a reflux condenser, $\mathrm{AlCl}_{3}$ ( 0.75 equiv) was dissolved in dry THF $\left(0.4 \mathrm{M}\right.$ against $\left.\mathrm{AlCl}_{3}\right)$ under an argon atmosphere. After all, $\mathrm{AlCl}_{3}$ was suspended, the mixture was cooled to $0{ }^{\circ} \mathrm{C}$ followed by a slow addition of a suspension of $\mathrm{LiAlH}_{4}$ (2.25 equiv) in THF (1.8 M against $\left.\mathrm{LiAlH}_{4}\right)$. After stirring for 15 minutes, the corresponding propargylic alcohol $\mathbf{S 3}$ (1.0 equiv) was added as a solution in THF (1.6 M against propargylic alcohol). The reaction mixture was heated to $80^{\circ} \mathrm{C}$ and stirred for $16 \mathrm{~h}$. It was cooled to $0{ }^{\circ} \mathrm{C}$ and quenched by slow addition of $\mathrm{H}_{2} \mathrm{O}(1 \mathrm{~mL}), 1 \mathrm{M} \mathrm{NaOH}$ aqueous solution $(2 \mathrm{~mL})$, and additional $\mathrm{H}_{2} \mathrm{O}(1 \mathrm{~mL})$. The precipitate was filtered with $\mathrm{Et}_{2} \mathrm{O}$, and the crude material was purified by silica gel column chromatography (typically, $\mathrm{Et}_{2} \mathrm{O} / \mathrm{hexane}$ 0:100-5:95) to obtain the corresponding 1,1-di-substituted allene product $\mathbf{1}$. 


\subsection{Preparation of 1-iodo-3-methoxypropane (2h). ${ }^{6,8}$}

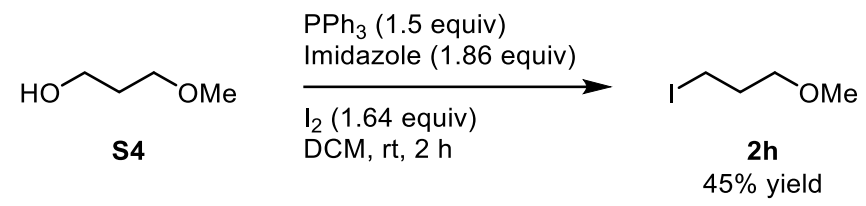

In a vacuum-dried, round-bottomed flask, $\mathrm{PPh}_{3}(3.93 \mathrm{~g}, 15.0 \mathrm{mmol}, 1.5$ equiv) and imidazole (1.27 g, $18.6 \mathrm{mmol}, 1.86$ equiv) were dissolved in dry DCM $(100 \mathrm{~mL}, 0.1 \mathrm{M})$ under a nitrogen atmosphere at rt. $\mathrm{I}_{2}$ (4.16 g, $16.4 \mathrm{mmol}, 1.64$ equiv) was then added to the reaction mixture, followed by addition of corresponding alcohol $\mathbf{S 4}$ (901 mg, $10 \mathrm{mmol}, 1.0$ equiv). After stirred for $2 \mathrm{~h}$ at rt, the reaction mixture was quenched by saturated $\mathrm{Na}_{2} \mathrm{~S}_{2} \mathrm{O}_{3}$ aqueous solution and extracted with DCM three times. The combined organic layer was washed with saturated $\mathrm{NaHCO}_{3}$ aqueous solution and brine, then dried over $\mathrm{MgSO}_{4}$. After filtration, the solvent was removed by evaporation. The crude mixture was purified by silica gel column chromatography (EtOAc/hexane $0: 100-5: 95$ ) to obtain the corresponding alkyl iodide $\mathbf{2 h}$ in $45 \%$ yield ( $902.8 \mathrm{mg}$, $4.5 \mathrm{mmol}$, colorless oil).

${ }^{1} \mathrm{H}$ NMR (392 MHz, $\left.\mathrm{CDCl}_{3}, \delta\right): 1.99-2.11$ (m, 2H), 3.21-3.31 (m, 2H), 3.31-3.38 (m, 3H), 3.39$3.49(\mathrm{~m}, 2 \mathrm{H}) .{ }^{13} \mathrm{C} \mathrm{NMR}\left(99 \mathrm{MHz}, \mathrm{CDCl}_{3}, \delta\right): 3.3\left(\mathrm{CH}_{2}\right), 33.3\left(\mathrm{CH}_{2}\right), 58.7\left(\mathrm{CH}_{3}\right), 71.9\left(\mathrm{CH}_{2}\right)$. HRMS$\mathrm{EI}(\mathrm{m} / \mathrm{z}):[\mathrm{M}]^{+}$calcd for $\mathrm{C}_{4} \mathrm{H}_{9} \mathrm{IO}, 199.9698$; found, 199.9701 . 


\section{Substrate Characterization}

Buta-2,3-dien-2-ylcyclohexane (1a). ${ }^{2}$<smiles>C=C=C(C)C1CCCCC1</smiles>

1a

1a was prepared from corresponding propargyl tosylate $(6.73 \mathrm{~g}, 30.0 \mathrm{mmol})$ according to the GP1. The product 1a was obtained in 67\% yield (2.73 g, $20.0 \mathrm{mmol}$, colorless oil).

${ }^{1} \mathrm{H}$ NMR $\left(392 \mathrm{MHz} \mathrm{CDCl}_{3}, \delta\right): 1.03-1.35(\mathrm{~m}, 5 \mathrm{H}), 1.60-1.86(\mathrm{~m}, 9 \mathrm{H}), 4.59$ (quintet, $J=2.9 \mathrm{~Hz}$, 2H). ${ }^{13} \mathrm{C} \mathrm{NMR}\left(99 \mathrm{MHz}, \mathrm{CDCl}_{3}, \delta\right): 17.1\left(\mathrm{CH}_{3}\right), 26.3\left(\mathrm{CH}_{2}\right), 26.5\left(\mathrm{CH}_{2}\right), 31.7\left(\mathrm{CH}_{2}\right), 40.9(\mathrm{CH}), 74.4$ $\left(C \mathrm{H}_{2}\right), 103.5(\mathrm{C}), 205.6(\mathrm{C})$. HRMS-EI $(\mathrm{m} / \mathrm{z})$ : $[\mathrm{M}]^{+}$calcd for $\mathrm{C}_{10} \mathrm{H}_{16}, 136.1252$; found, 136.1253 .

\section{3,4,4-Trimethylpenta-1,2-diene (1b). ${ }^{3}$}

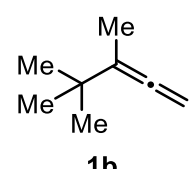

1b was prepared from corresponding propargyl tosylate $(6.73 \mathrm{~g}, 30.0 \mathrm{mmol})$ according to the GP1. The product $\mathbf{1 b}$ was obtained in $37 \%$ yield (1.21 g, $11.0 \mathrm{mmol}$, colorless oil).

${ }^{1} \mathrm{H}$ NMR $\left(392 \mathrm{MHz}, \mathrm{CDCl}_{3}, \delta\right): 1.05(\mathrm{~s}, 9 \mathrm{H}), 1.69(\mathrm{t}, J=3.1 \mathrm{~Hz}, 3 \mathrm{H}), 4.54-4.60(\mathrm{~m}, 2 \mathrm{H}) .{ }^{13} \mathrm{C}$ NMR (99 MHz, $\left.\mathrm{CDCl}_{3}, \delta\right): 14.8\left(\mathrm{CH}_{3}\right), 28.9\left(\mathrm{CH}_{3}\right), 32.6(C), 74.2\left(\mathrm{CH}_{2}\right), 107.2(C), 205.2(C)$. HRMS-EI $(\mathrm{m} / \mathrm{z}):[\mathrm{M}]^{+}$calcd for $\mathrm{C}_{8} \mathrm{H}_{14}, 110.1096$; found, 110.1092 .

1-(tert-Butyl)-4-(2,3-dimethylpenta-3,4-dien-1-yl)-benzene (1c).

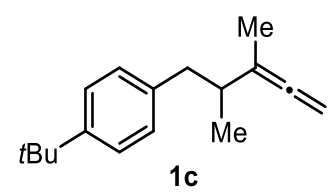

1c was prepared from corresponding propargyl tosylate (1.92 g, $5.00 \mathrm{mmol})$ according to the GP1. The product 1c was obtained in $81 \%$ yield (922 $\mathrm{mg}, 4.04 \mathrm{mmol}$, colorless oil).

${ }^{1} \mathrm{H}$ NMR (392 MHz, $\left.\mathrm{CDCl}_{3}, \delta\right): 0.98(\mathrm{~d}, J=6.7 \mathrm{~Hz}, 3 \mathrm{H}), 1.31(\mathrm{~s}, 9 \mathrm{H}), 1.71(\mathrm{t}, J=3.1 \mathrm{~Hz}, 3 \mathrm{H})$, 2.18-2.28 (m, 1H), 2.36-2.44 (m, 1H), 2.79-2.86 (m, 1H), 4.56-4.62 (m, 2H), 7.06-7.11 (m, 2H), 7.24-7.31 (m, 2H). ${ }^{13} \mathrm{C} \mathrm{NMR}\left(99 \mathrm{MHz}, \mathrm{CDCl}_{3}, \delta\right): 17.1\left(\mathrm{CH}_{3}\right), 18.7\left(\mathrm{CH}_{3}\right), 31.4\left(\mathrm{CH}_{3}\right), 34.3(C), 38.7$ $(\mathrm{CH}), 41.2\left(\mathrm{CH}_{2}\right), 74.8\left(\mathrm{CH}_{2}\right), 103.0(C), 124.9(\mathrm{CH}), 128.7(\mathrm{CH}), 138.0(C), 148.5(C), 205.7(C)$. HRMS-EI $(m / z):[\mathrm{M}-\mathrm{Me}]^{+}$calcd for $\mathrm{C}_{16} \mathrm{H}_{21}, 213.1643$; found, 213.1643. 
1-(tert-Butyl)-4-(2-methyl-3-vinylidenehexyl)-benzene (1d).

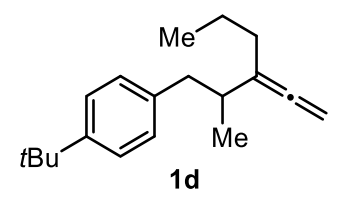

1d was prepared from corresponding propargyl tosylate $(1.92 \mathrm{~g}, 5.00 \mathrm{mmol})$ according to the GP1. The product 1d was obtained in 92\% yield (1.18 g, $4.62 \mathrm{mmol}$, colorless oil).

${ }^{1} \mathrm{H} \mathrm{NMR}\left(392 \mathrm{MHz}, \mathrm{CDCl}_{3}, \delta\right): 0.88$ (t, $\left.J=7.4 \mathrm{~Hz}, 3 \mathrm{H}\right), 0.98$ (d, $\left.J=6.7 \mathrm{~Hz}, 3 \mathrm{H}\right), 1.30$ (s, 9H), $1.37-1.49(\mathrm{~m}, 2 \mathrm{H}), 1.82-2.00(\mathrm{~m}, 2 \mathrm{H}), 2.09-2.26(\mathrm{~m}, 1 \mathrm{H}), 2.31-2.47(\mathrm{~m}, 1 \mathrm{H}), 2.75-2.90(\mathrm{~m}, 1 \mathrm{H})$, 4.63-4.71 (m, 2H), 7.04-7.14 (m, 2H), 7.23-7.31 (m, 2H). $\left.{ }^{13} \mathrm{C} \mathrm{NMR} \mathrm{(99} \mathrm{MHz,} \mathrm{CDCl}_{3}, \delta\right): 13.9\left(\mathrm{CH}_{3}\right)$, $19.1\left(\mathrm{CH}_{3}\right), 20.9\left(\mathrm{CH}_{2}\right), 31.4\left(\mathrm{CH}_{3}\right), 32.9\left(\mathrm{CH}_{2}\right), 34.3(\mathrm{C}), 38.0(\mathrm{CH}), 41.7\left(\mathrm{CH}_{2}\right), 76.8\left(\mathrm{CH}_{2}\right), 108.2$ (C), $124.9(\mathrm{CH}), 128.8(\mathrm{CH}), 138.2(\mathrm{C}), 148.4(\mathrm{C}), 205.3(\mathrm{C})$. HRMS-EI $(\mathrm{m} / \mathrm{z})$ : [M-Me] ${ }^{+}$calcd for $\mathrm{C}_{18} \mathrm{H}_{25}, 241.1956$; found, 241.1948 .

\section{1-(tert-Butyl)-4-(2-methyl-3-vinylideneundecyl)-benzene (1e).}

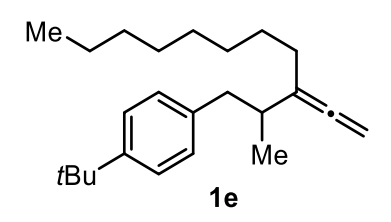

1e was prepared from corresponding propargyl tosylate $(1.15 \mathrm{~g}, 3.00 \mathrm{mmol})$ according to the GP1. The product 1e was obtained in $75 \%$ yield $(587 \mathrm{mg}, 1.79 \mathrm{mmol}$, colorless oil).

${ }^{1} \mathrm{H}$ NMR (392 MHz, $\mathrm{CDCl}_{3}, \delta$ ): 0.88 (t, $\left.J=6.9 \mathrm{~Hz}, 3 \mathrm{H}\right), 0.97$ (d, $\left.J=6.4 \mathrm{~Hz}, 3 \mathrm{H}\right), 1.19-1.48$ (m, $12 \mathrm{H}), 1.31$ (s, 9H), 1.86-1.99 (m, 2H), 2.12-2.24 (m, 1H), 2.39 (dd, $J=13.3,8.7 \mathrm{~Hz}, 1 \mathrm{H}), 2.82$ (dd, $J=13.3,5.5 \mathrm{~Hz}, 1 \mathrm{H}), 4.66-4.70(\mathrm{~m}, 2 \mathrm{H}), 7.05-7.11(\mathrm{~m}, 2 \mathrm{H}), 7.24-7.31$ (m, 2H). ${ }^{13} \mathrm{C}$ NMR $(99 \mathrm{MHz}$, $\left.\mathrm{CDCl}_{3}, \delta\right): 14.1\left(\mathrm{CH}_{3}\right), 19.1\left(\mathrm{CH}_{3}\right), 22.7\left(\mathrm{CH}_{2}\right), 27.6\left(\mathrm{CH}_{2}\right), 29.3\left(\mathrm{CH}_{3}\right), 29.4\left(\mathrm{CH}_{3}\right), 29.5\left(\mathrm{CH}_{3}\right), 30.7$ $\left(\mathrm{CH}_{2}\right), 31.4\left(\mathrm{CH}_{3}\right), 31.9\left(\mathrm{CH}_{2}\right), 34.3(\mathrm{C}), 37.9(\mathrm{CH}), 41.6\left(\mathrm{CH}_{2}\right), 76.8\left(\mathrm{CH}_{2}\right), 108.5(\mathrm{C}), 124.9(\mathrm{CH})$, $128.8(\mathrm{CH}), 138.2(\mathrm{C}), 148.4(\mathrm{C}), 205.2(\mathrm{C})$. HRMS-EI $(\mathrm{m} / \mathrm{z})$ : $[\mathrm{M}-\mathrm{Me}]^{+}$calcd for $\mathrm{C}_{23} \mathrm{H}_{35}, 311.2739$; found, 311.2732 . 
1-(tert-Butyl)-4-(2,5-dimethyl-3-vinylidenehexyl)-benzene (1f).

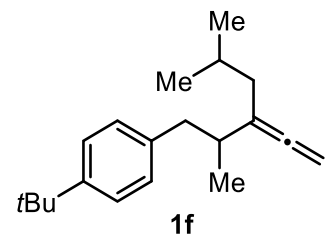

1f was prepared from corresponding propargyl tosylate $(1.15 \mathrm{~g}, 3.00 \mathrm{mmol})$ according to the GP1. The product $1 \mathbf{f}$ was obtained in $83 \%$ yield $(674 \mathrm{mg}, 2.49 \mathrm{mmol}$, colorless oil).

${ }^{1} \mathrm{H}$ NMR $\left(392 \mathrm{MHz}, \mathrm{CDCl}_{3}, \delta\right): 0.85(\mathrm{~d}, J=6.4 \mathrm{~Hz}, 3 \mathrm{H}), 0.88(\mathrm{~d}, J=6.0 \mathrm{~Hz}, 3 \mathrm{H}), 0.97$ (d, $J=6.9$ $\mathrm{Hz}, 3 \mathrm{H}), 1.31$ (s, 9H), 1.65-1.78 (m, 1H), 1.79-1.86 (m, 2H), 2.09-2.20 (m, 1H), 2.39 (dd, $J=13.8$, $8.7 \mathrm{~Hz}, 1 \mathrm{H}), 2.81(\mathrm{dd}, J=13.3,6.0 \mathrm{~Hz}, 1 \mathrm{H}), 4.65-4.71(\mathrm{~m}, 2 \mathrm{H}), 7.05-7.12(\mathrm{~m}, 2 \mathrm{H}), 7.24-7.31(\mathrm{~m}$, 2H). ${ }^{13} \mathrm{C} \mathrm{NMR}\left(99 \mathrm{MHz}, \mathrm{CDCl}_{3}, \delta\right): 19.1\left(\mathrm{CH}_{3}\right), 22.56\left(\mathrm{CH}_{3}\right), 22.63\left(\mathrm{CH}_{3}\right), 26.6(\mathrm{CH}), 31.4\left(\mathrm{CH}_{3}\right)$, $34.3(\mathrm{C}), 37.9(\mathrm{CH}), 40.5\left(\mathrm{CH}_{2}\right), 41.6\left(\mathrm{CH}_{2}\right), 76.4\left(\mathrm{CH}_{2}\right), 107.2(\mathrm{C}), 124.9(\mathrm{CH}), 128.8(\mathrm{CH}), 138.2$ (C), $148.4(C), 205.8(C)$. HRMS-EI $(m / z)$ : [M-Me] ${ }^{+}$calcd for $\mathrm{C}_{19} \mathrm{H}_{27}, 255.2113$; found, 255.2115.

1-(Buta-2,3-dien-2-yl)-adamantane (1g). ${ }^{4}$

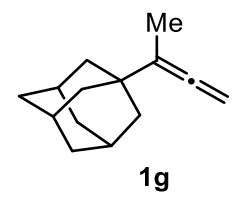

$1 \mathrm{~g}$ was prepared from corresponding propargylic $(1.28 \mathrm{~g}, 6.28 \mathrm{mmol})$ alcohol according to the GP2. The product $1 \mathrm{~g}$ was obtained in 39\% yield (456 mg, $2.42 \mathrm{mmol}$, colorless oil) via GPC purification.

${ }^{1} \mathrm{H}$ NMR (392 MHz, $\left.\mathrm{CDCl}_{3}, \delta\right): 1.55-1.77$ (m, 15H), 1.99 (s, 3H), 4.55-4.62 (m, 2H). ${ }^{13} \mathrm{C}$ NMR (99 MHz, $\left.\mathrm{CDCl}_{3}, \delta\right): 13.4\left(\mathrm{CH}_{3}\right), 28.8(\mathrm{CH}), 34.2(\mathrm{C}), 36.9\left(\mathrm{CH}_{2}\right), 41.1\left(\mathrm{CH}_{2}\right), 74.4\left(\mathrm{CH}_{2}\right), 107.6(C)$, $205.7(C)$. HRMS-EI $(m / z)$ : $[\mathrm{M}]^{+}$calcd for $\mathrm{C}_{14} \mathrm{H}_{20}, 188.1565$; found, 188.1565 .

3-(tert-Butyl)-hepta-1,2-diene (1h).

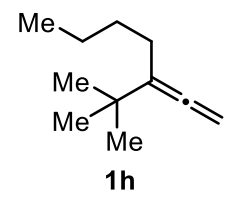

1h was prepared from corresponding propargyl tosylate $(7.13 \mathrm{~g}, 27.0 \mathrm{mmol})$ according to the GP1. The product $1 \mathrm{~h}$ was obtained in $69 \%$ yield $(2.83 \mathrm{~g}, 18.6 \mathrm{mmol}$, colorless oil).

${ }^{1} \mathrm{H}$ NMR (392 MHz, $\left.\mathrm{CDCl}_{3}, \delta\right): 0.91$ (t, $\left.J=7.3 \mathrm{~Hz}, 3 \mathrm{H}\right), 1.04$ (s, 9H), 1.29-1.44 (m, 4H), 1.87$1.95(\mathrm{~m}, 2 \mathrm{H}), 4.68(\mathrm{t}, J=5.9 \mathrm{~Hz}, 2 \mathrm{H}) .{ }^{13} \mathrm{C} \mathrm{NMR}\left(99 \mathrm{MHz}, \mathrm{CDCl}_{3}, \delta\right): 14.1\left(\mathrm{CH}_{3}\right), 22.6\left(\mathrm{CH}_{2}\right), 26.2$ $\left(\mathrm{CH}_{2}\right), 29.2\left(\mathrm{CH}_{3}\right), 30.5\left(\mathrm{CH}_{2}\right), 32.8(\mathrm{C}), 76.8\left(\mathrm{CH}_{2}\right), 112.6(\mathrm{C}), 204.5(\mathrm{C})$. HRMS-EI $(\mathrm{m} / \mathrm{z}):[\mathrm{M}-\mathrm{Me}]^{+}$ calcd for $\mathrm{C}_{10} \mathrm{H}_{17}, 137.1330$; found, 137.1329 . 
(4-Methylhexa-4,5-dien-1-yl)-benzene (1i).<smiles>C=C(C)CCCc1ccccc1</smiles>

$1 \mathrm{i}$

1i was prepared from corresponding propargyl tosylate $(6.36 \mathrm{~g}, 19.3 \mathrm{mmol})$ according to the GP1. The product $1 \mathrm{i}$ was obtained in $76 \%$ yield $(2.54 \mathrm{~g}, 14.7 \mathrm{mmol}$, colorless oil).

${ }^{1} \mathrm{H}$ NMR $\left(396 \mathrm{MHz}, \mathrm{CDCl}_{3}, \delta\right.$ ): 1.68 (t, $\left.J=3.2 \mathrm{~Hz}, 3 \mathrm{H}\right), 1.76$ (quintet, $J=7.4 \mathrm{~Hz}, 2 \mathrm{H}$ ), 1.91-2.02 (m, 2H), $2.63(\mathrm{t}, J=7.7 \mathrm{~Hz}, 2 \mathrm{H}), 4.61$ (sextet, $J=3.1 \mathrm{~Hz}, 2 \mathrm{H}), 7.13-7.22(\mathrm{~m}, 3 \mathrm{H}), 7.24-7.32(\mathrm{~m}, 2 \mathrm{H})$. ${ }^{13} \mathrm{C}$ NMR $\left(100 \mathrm{MHz}, \mathrm{CDCl}_{3}, \delta\right): 18.8\left(\mathrm{CH}_{3}\right), 29.2\left(\mathrm{CH}_{2}\right), 32.8\left(\mathrm{CH}_{2}\right), 35.4\left(\mathrm{CH}_{2}\right), 74.2\left(\mathrm{CH}_{2}\right), 98.1$ (C), $125.6(\mathrm{CH}), 128.2(\mathrm{CH}), 128.5(\mathrm{CH}), 142.5(\mathrm{C}), 206.1(\mathrm{C})$. HRMS-EI $(\mathrm{m} / \mathrm{z})$ : $[\mathrm{M}]^{+}$calcd for $\mathrm{C}_{13} \mathrm{H}_{16}$, 172.1252; found, 172.1252 .

\section{3-Methylundeca-1,2-diene (1j).}

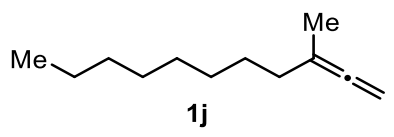

1j was prepared from corresponding propargyl tosylate $(3.36 \mathrm{~g}, 15.0 \mathrm{mmol})$ according to the GP1. The product $\mathbf{1} \mathbf{j}$ was obtained in $80 \%$ yield $(1.99 \mathrm{~g}, 12.0 \mathrm{mmol}$, colorless oil).

${ }^{1} \mathrm{H}$ NMR (392 MHz, $\left.\mathrm{CDCl}_{3}, \delta\right): 0.88(\mathrm{t}, J=6.7 \mathrm{~Hz}, 3 \mathrm{H}), 1.20-1.35(\mathrm{~m}, 10 \mathrm{H}), 1.36-1.47(\mathrm{~m}, 2 \mathrm{H})$, $1.67(\mathrm{t}, J=3.1 \mathrm{~Hz}, 3 \mathrm{H}), 1.87-1.96(\mathrm{~m}, 2 \mathrm{H}), 4.57$ (sextet, $J=3.1 \mathrm{~Hz}, 2 \mathrm{H}) .{ }^{13} \mathrm{C}$ NMR $\left(99 \mathrm{MHz}, \mathrm{CDCl}_{3}\right.$,

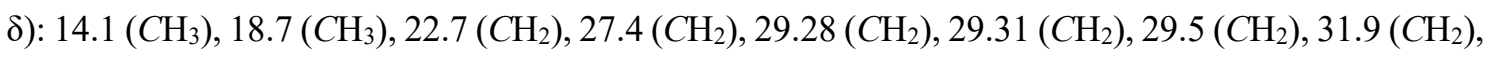
$33.5\left(\mathrm{CH}_{2}\right), 73.7\left(\mathrm{CH}_{2}\right), 98.5(\mathrm{C}), 206.1(\mathrm{C})$. HRMS-EI $(\mathrm{m} / \mathrm{z})$ : [M] ${ }^{+}$calcd for $\mathrm{C}_{12} \mathrm{H}_{22}, 166.1722$; found, 166.1723 .

(2-Ethylbuta-2,3-dien-1-yl)benzene (1k).

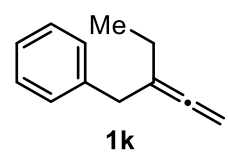

$1 \mathbf{k}$ was prepared from corresponding propargyl tosylate $(6.91 \mathrm{~g}, 29.0 \mathrm{mmol})$ according to the GP1. The product $1 \mathbf{k}$ was obtained in $9 \%$ yield ( $427 \mathrm{mg}, 2.70 \mathrm{mmol}$, colorless oil) via GPC purification.

${ }^{1} \mathrm{H}$ NMR (401 MHz, $\left.\mathrm{CDCl}_{3}, \delta\right): 0.99$ (t, $\left.J=7.2 \mathrm{~Hz}, 3 \mathrm{H}\right), 1.85-1.95$ (m, 2H), 3.31 (s, 2H), 4.66$4.73(\mathrm{~m}, 2 \mathrm{H}), 7.16-7.24(\mathrm{~m}, 3 \mathrm{H}), 7.24-7.32(\mathrm{~m}, 2 \mathrm{H}) .{ }^{13} \mathrm{C} \mathrm{NMR}\left(99 \mathrm{MHz}, \mathrm{CDCl}_{3}, \delta\right): 12.1\left(\mathrm{CH}_{3}\right), 24.1$ $\left(\mathrm{CH}_{2}\right), 39.6\left(\mathrm{CH}_{2}\right), 75.7\left(\mathrm{CH}_{2}\right), 104.4(\mathrm{C}), 126.1(\mathrm{CH}), 128.2(\mathrm{CH}), 128.9(\mathrm{CH}), 139.8(\mathrm{C}), 206.4(\mathrm{C})$. HRMS-EI $(m / z):[\mathrm{M}]^{+}$calcd for $\mathrm{C}_{12} \mathrm{H}_{14}, 158.1096$; found, 158.1091 . 
(4-Vinylidenecyclohexyl)benzene (11). ${ }^{5}$

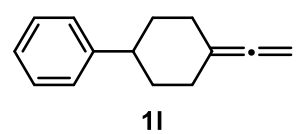

11 was prepared from corresponding propargylic alcohol $(1.60 \mathrm{~g}, 8.00 \mathrm{mmol})$ according to the GP2. The product 11 was obtained in $19 \%$ yield ( $274 \mathrm{mg}, 1.49 \mathrm{mmol}$, colorless oil) via GPC purification.

${ }^{1} \mathrm{H}$ NMR (392 MHz, $\mathrm{CDCl}_{3}, \delta$ ): 1.52-1.70 (m, 2H), 1.93-2.03 (m, 2H), 2.11-2.25 (m, 2H), 2.39$2.49(\mathrm{~m}, 2 \mathrm{H}), 2.53-2.64(\mathrm{~m}, 1 \mathrm{H}), 4.54-4.66(\mathrm{~m}, 2 \mathrm{H}), 7.15-7.24(\mathrm{~m}, 3 \mathrm{H}), 7.26-7.34(\mathrm{~m}, 2 \mathrm{H}) .{ }^{13} \mathrm{C} \mathrm{NMR}$ (100 MHz, $\left.\mathrm{CDCl}_{3}, \delta\right): 31.0\left(\mathrm{CH}_{2}\right), 34.4\left(\mathrm{CH}_{2}\right), 43.9(\mathrm{CH}), 72.9\left(\mathrm{CH}_{2}\right), 100.0(\mathrm{C}), 126.0(\mathrm{CH}), 126.8$ $(\mathrm{CH}), 128.4(\mathrm{CH}), 146.8(\mathrm{C}), 203.5(\mathrm{C})$. HRMS-EI $(\mathrm{m} / \mathrm{z})$ : [M] ${ }^{+}$calcd for $\mathrm{C}_{14} \mathrm{H}_{16}, 184.1252$; found, 184.1251 .

$(8 S, 9 S, 13 S, 14 S)$-3-Methoxy-13-methyl-17-vinylidene-7,8,9,11,12,13,14,15,16,17-decahydro-6Hcyclopenta $[a]$ phenanthrene $(1 \mathrm{~m}){ }^{4}$

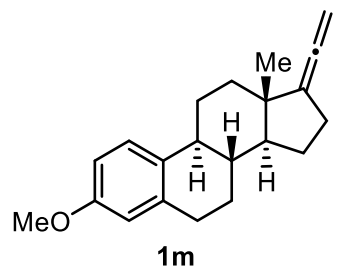

$1 \mathrm{~m}$ was prepared from corresponding propargylic alcohol $(1.55 \mathrm{~g}, 5.00 \mathrm{mmol})$ according to the GP2. The product $1 \mathrm{~m}$ was obtained in $34 \%$ yield $(505 \mathrm{mg}, 1.72 \mathrm{mmol}$, white solid).

${ }^{1} \mathrm{H}$ NMR $\left(392 \mathrm{MHz}, \mathrm{CDCl}_{3}, \delta\right): 0.90$ (s, 3H), 1.18-1.65 (m, 6H), 1.74-2.01 (m, 3H), 2.14-2.51 (m, $3 \mathrm{H}), 2.53-2.68(\mathrm{~m}, 1 \mathrm{H}), 2.78-2.97(\mathrm{~m}, 2 \mathrm{H}), 3.78(\mathrm{~s}, 3 \mathrm{H}), 4.66-4.79(\mathrm{~m}, 2 \mathrm{H}), 6.63(\mathrm{~d}, J=2.7 \mathrm{~Hz}, 1 \mathrm{H})$, $6.71(\mathrm{dd}, J=8.4,2.5 \mathrm{~Hz}, 1 \mathrm{H}), 7.22(\mathrm{~d}, J=8.6 \mathrm{~Hz}, 1 \mathrm{H}) .{ }^{13} \mathrm{C} \mathrm{NMR}\left(99 \mathrm{MHz}, \mathrm{CDCl}_{3}, \delta\right): 18.4\left(\mathrm{CH}_{3}\right)$, $24.5\left(\mathrm{CH}_{2}\right), 26.6\left(\mathrm{CH}_{2}\right), 27.3\left(\mathrm{CH}_{2}\right), 27.7\left(\mathrm{CH}_{2}\right), 29.8\left(\mathrm{CH}_{2}\right), 36.0\left(\mathrm{CH}_{2}\right), 38.7(\mathrm{CH}), 43.9(\mathrm{CH}), 44.4$ $(C), 54.4(\mathrm{CH}), 55.2\left(\mathrm{CH}_{3}\right), 76.8\left(\mathrm{CH}_{2}\right), 111.4(\mathrm{CH}), 111.9(\mathrm{C}), 113.7(\mathrm{CH}), 126.3(\mathrm{CH}), 132.7(\mathrm{C})$, $137.9(C), 157.4(C), 200.4(C)$. HRMS-EI $(m / z)$ : $[\mathrm{M}]^{+}$calcd for $\mathrm{C}_{21} \mathrm{H}_{26} \mathrm{O}, 294.1984$; found, 294.1982. 


\section{General Borylation Procedure}

\subsection{Procedure for the copper(I)-catalyzed alkyl borylation of 1a (Optimized Conditions $A=$ GP3).}

SIMesCuCl was prepared according to the literature. ${ }^{9}$ SIMesCuCl $(4.1 \mathrm{mg}, 0.010 \mathrm{mmol})$, bis(pinacolato)diboron (3) (152.4 mg, $0.60 \mathrm{mmol})$, and $\mathrm{K}(\mathrm{O}-\mathrm{t}$ - $\mathrm{Bu})(67.3 \mathrm{mg}, 0.60 \mathrm{mmol})$ were placed in an oven-dried reaction vial in an argon-filled glove box. After the vial was sealed with a screw cap containing a Teflon ${ }^{\mathrm{TM}}$-coated rubber septum and taken from the glove box, dry DMF $(1.0 \mathrm{~mL}) \mathrm{was}$ added to the vial through the rubber septum using a syringe. After stirred for $10 \mathrm{~min}$, the reaction mixture was cooled to $-5{ }^{\circ} \mathrm{C}$ and stirred for $10 \mathrm{~min}$. Then $1 \mathbf{a}(0.50 \mathrm{mmol})$ and $\mathbf{2 a}(1.0 \mathrm{mmol})$ were added to the mixture. The reaction mixture was stirred at $-5{ }^{\circ} \mathrm{C}$ for $24 \mathrm{~h}$. The reaction monitoring and regioselectivity determination were done by GC analysis. After the reaction was completed, the reaction mixture was passed through a short silica gel column $(\Phi: 10 \mathrm{~mm}$, the height of the silica-gel column: $30 \mathrm{~mm}$ ) eluting with $\mathrm{Et}_{2} \mathrm{O} /$ hexane $(5 / 95)$. The crude material was purified by flash column chromatography $\left(\mathrm{SiO}_{2}, \mathrm{Et}_{2} \mathrm{O} /\right.$ hexane, 0:100-3:97) to give the corresponding allyl boronate $(E)-4 a a$. In order to remove an undesired boryl substitution product of the alkyl halide and protoboration product of the allene for the further purification and the determination of the regio- and stereoselectivities, $(E)$ 4aa was treated with $\mathrm{NaBO}_{3} \bullet 4 \mathrm{H}_{2} \mathrm{O}$ as an oxidant for the boryl group in $\mathrm{THF} / \mathrm{H}_{2} \mathrm{O}(1: 1)$. The mixture was stirred at room temperature overnight. After the reaction complete, the reaction mixture was extracted with $\mathrm{Et}_{2} \mathrm{O}$ and dried over $\mathrm{MgSO}_{4}$. The solvents were removed under reduced pressure. The crude material was purified by flash column chromatography $\left(\mathrm{SiO}_{2}\right.$, EtOAc/hexane, 0:100-12:88) to give the corresponding alcohol product (Z)-6aa as a colorless oil. Then, the regioselectivity and stereoselectivity were determined by ${ }^{1} \mathrm{H}$ and ${ }^{13} \mathrm{C}$ NMR analysis.

\subsection{Procedure for the copper(I)-catalyzed alkyl borylation of 1 b (Optimized Conditions B = GP4).}

$\mathrm{CuCl}$ (2.5 mg, $0.025 \mathrm{mmol})$, Xantphos (14.5 mg, $0.025 \mathrm{mmol})$, bis(pinacolato)diboron (3) (152.4 $\mathrm{mg}, 0.60 \mathrm{mmol})$, and $\mathrm{K}(\mathrm{O}-\mathrm{t}-\mathrm{Bu})(67.3 \mathrm{mg}, 0.60 \mathrm{mmol})$ were placed in an oven-dried reaction vial in an argon-filled glove box. After the vial was sealed with a screw cap containing a Teflon ${ }^{\mathrm{TM}}$-coated rubber septum and taken from the glove box, dry DMF $(1.0 \mathrm{~mL})$ was added to the vial through the rubber septum using a syringe. After stirring for $10 \mathrm{~min}, \mathbf{1 b}(0.50 \mathrm{mmol})$ and $\mathbf{2 a}(1.0 \mathrm{mmol})$ were added to the mixture. The reaction mixture was stirred at $30^{\circ} \mathrm{C}$ for $24 \mathrm{~h}$. The reaction monitoring was done by GC analysis. After the reaction was completed, the reaction mixture was passed through a short silica gel column ( $\Phi: 10 \mathrm{~mm}$, the height of the silica-gel column: $30 \mathrm{~mm}$ ) eluting with $\mathrm{Et}_{2} \mathrm{O} /$ hexane (5/95). The crude material was purified by flash column chromatography $\left(\mathrm{SiO}_{2}\right.$, $\mathrm{Et}_{2} \mathrm{O} /$ hexane, 0:100-3:97) to give the corresponding allyl boronate $(E)-\mathbf{4 b a}$. Then, the regioselectivity and stereoselectivity were determined by ${ }^{1} \mathrm{H}$ NMR analysis. 


\section{Borylation Product Characterization}

(Z)-2-(1-Cyclohexylethylidene)-6,6,6-trifluorohexan-1-ol [(Z)-6aa].<smiles>CC(CCCC(F)(F)F)=C(CB1OC(C)(C)C(C)(C)O1)C1CCCCC1</smiles>

(E)-4aa<smiles>CC(=C(CO)CCCC(F)(F)F)C1CCCCC1</smiles>

(Z)-6aa

The reaction was conducted with $68.0 \mathrm{mg}(0.499 \mathrm{mmol})$ of 1 a according to the GP3. The borylation product $(E)$-4aa was obtained in 77\% yield with $E / Z=<5: 95,4: 5=>95: 5$ (determined by GC and ${ }^{13} \mathrm{C}$ NMR analysis). In order to remove an undesired boryl substitution product of the alkyl halide and protoboration product of the allene for the further purification, the oxidation of the boryl groups was performed. The product (Z)-6aa was obtained in 66\% yield with $E / Z=<5: 95,4: 5=>95: 5(86.4 \mathrm{mg}$, $0.327 \mathrm{mmol}$, colorless oil).

${ }^{1} \mathrm{H}$ NMR (392 MHz, $\left.\mathrm{CDCl}_{3}, \delta\right): 1.07-1.52(\mathrm{~m}, 8 \mathrm{H}), 1.55-1.85(\mathrm{~m}, 5 \mathrm{H}), 1.60(\mathrm{~s}, 3 \mathrm{H}), 1.99-2.16(\mathrm{~m}$, 2H), $2.20(\mathrm{t}, J=8.0 \mathrm{~Hz}, 2 \mathrm{H}), 2.46-2.63(\mathrm{~m}, 1 \mathrm{H}), 4.14(\mathrm{~s}, 2 \mathrm{H}) .{ }^{13} \mathrm{C} \mathrm{NMR}\left(99 \mathrm{MHz}, \mathrm{CDCl}_{3}, \delta\right): 13.5$ $\left(\mathrm{CH}_{3}\right), 20.8-21.2\left(\mathrm{~m}, \mathrm{CH}_{2}\right), 26.0\left(\mathrm{CH}_{2}\right), 26.4\left(\mathrm{CH}_{2}\right), 30.1\left(\mathrm{CH}_{2}\right), 31.4\left(\mathrm{CH}_{2}\right), 33.5(\mathrm{q}, J=28.6 \mathrm{~Hz}$, $\left.\mathrm{CH}_{2}\right), 41.1(\mathrm{CH}), 61.1\left(\mathrm{CH}_{2}\right), 127.2(\mathrm{q}, J=277.6 \mathrm{~Hz}, C), 130.2(C), 140.6(C)$. HRMS-EI $(m / z)$ : $[\mathrm{M}]^{+}$ calcd for $\mathrm{C}_{14} \mathrm{H}_{23} \mathrm{~F}_{3} \mathrm{O}$, 264.1701; found, 264.1700.

\section{(E)-2-[2-(3,3-Dimethylbutan-2-ylidene)-6,6,6-trifluorohexyl]-4,4,5,5-tetramethyl-1,3,2-} dioxaborolane $[(E)-4 b a]$.

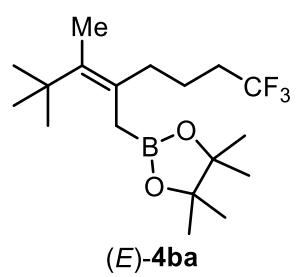

The reaction was conducted with $55.1 \mathrm{mg}(0.500 \mathrm{mmol})$ of $\mathbf{1 b}$ according to the GP4. The product (E)-4ba was obtained in 83\% yield with $E / Z=>95: 5,4: 5=>95: 5$ (144.0 $\mathrm{mg}, 0.413 \mathrm{mmol}$, colorless oil).

${ }^{1} \mathrm{H}$ NMR (396 MHz, $\left.\mathrm{CDCl}_{3}, \delta\right): 1.14(\mathrm{~s}, 9 \mathrm{H}), 1.23(\mathrm{~s}, 12 \mathrm{H}), 1.57-1.68(\mathrm{~m}, 5 \mathrm{H}), 1.84(\mathrm{~s}, 2 \mathrm{H}), 1.98-$ $2.12(\mathrm{~m}, 4 \mathrm{H}) .{ }^{13} \mathrm{C}$ NMR (100 MHz, $\left.\mathrm{CDCl}_{3}, \delta\right): 17.0\left(\mathrm{CH}_{3}\right), 19.3-20.9\left(\mathrm{~m}, \mathrm{CH}_{2}\right.$ and br, B-CH$), 24.6$ $\left(\mathrm{CH}_{3}\right), 30.7\left(\mathrm{CH}_{3}\right), 33.7\left(\mathrm{q}, J=28.4 \mathrm{~Hz}, \mathrm{CH}_{2}\right), 35.7(C), 36.5\left(C \mathrm{H}_{2}\right), 83.0(C), 127.3(\mathrm{q}, J=277.5 \mathrm{~Hz}$, C), $128.1(C), 135.3$ (C). ${ }^{11} \mathrm{~B}$ NMR (126 MHz, $\mathrm{CDCl}_{3}, \delta$ ): 33.0 (br, s). HRMS-EI (m/z): [M] ${ }^{+}$calcd for $\mathrm{C}_{18} \mathrm{H}_{32}{ }^{11} \mathrm{BF}_{3} \mathrm{O}_{2}, 348.2451$; found, 348.2445. 
(Z)-3-Cyclohexyl-2-ethylbut-2-en-1-ol [(Z)-6ab].

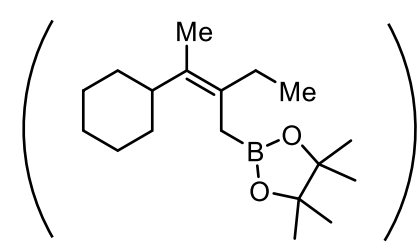

(E)-4ab<smiles>CCC(CO)=C(CO)C1CCCCC1</smiles>

(Z)-6ab

The reaction was conducted with $68.0 \mathrm{mg}(0.499 \mathrm{mmol})$ of $\mathbf{1 a}$ according to the GP3. In order to remove an undesired boryl substitution product of the alkyl halide and protoboration product of the allene for the further purification and the determination of the regio- and stereoselectivities, the oxidation of the boryl groups was performed. The product (Z)-6ab was obtained in $62 \%$ yield with $E / Z=<5: 95,4: 5=>95: 5(56.5 \mathrm{mg}, 0.310 \mathrm{mmol}$, colorless oil $)$.

${ }^{1} \mathrm{H}$ NMR (392 MHz, $\left.\mathrm{CDCl}_{3}, \delta\right): 0.97$ (t, $\left.J=7.6 \mathrm{~Hz}, 3 \mathrm{H}\right), 1.07-1.22(\mathrm{~m}, 2 \mathrm{H}), 1.23-1.48(\mathrm{~m}, 6 \mathrm{H})$, $1.56(\mathrm{~s}, 3 \mathrm{H}), 1.62-1.86(\mathrm{~m}, 3 \mathrm{H}), 2.14(\mathrm{q}, J=7.6 \mathrm{~Hz}, 2 \mathrm{H}), 2.48-2.61(\mathrm{~m}, 1 \mathrm{H}), 4.13(\mathrm{~s}, 2 \mathrm{H}) .{ }^{13} \mathrm{C} \mathrm{NMR}$ (99 MHz, $\left.\mathrm{CDCl}_{3}, \delta\right): 13.21\left(\mathrm{CH}_{3}\right), 13.24\left(\mathrm{CH}_{3}\right), 24.3\left(\mathrm{CH}_{2}\right), 26.1\left(\mathrm{CH}_{2}\right), 26.5\left(\mathrm{CH}_{2}\right), 31.5\left(\mathrm{CH}_{2}\right), 41.0$ $(\mathrm{CH}), 61.2\left(\mathrm{CH}_{2}\right), 133.2(\mathrm{C}), 138.9(\mathrm{C})$. HRMS-EI $(\mathrm{m} / \mathrm{z})$ : [M] ${ }^{+}$calcd for $\mathrm{C}_{12} \mathrm{H}_{22} \mathrm{O}, 182.1671$; found, 182.1673 .

\section{(Z)-2-(1-Cyclohexylethylidene)-heptan-1-ol [(Z)-6ac].}<smiles>CCCCC(CB1OC(C)(C)C(C)(C)O1)=C(C)C1CCCCC1</smiles>

$(E)-4 a c$<smiles>CCCCC/C(CO)=C(\C)C1CCCCC1</smiles>

(Z)-6ac

The reaction was conducted with $68.0 \mathrm{mg}(0.499 \mathrm{mmol})$ of 1 a according to the GP3. In order to remove an undesired boryl substitution product of the alkyl halide and protoboration product of the allene for the further purification and the determination of the regio- and stereoselectivities, the oxidation of the boryl groups was performed. The product $(Z)-6$ ac was obtained in $49 \%$ yield with $E / Z$ $=<5: 95,4: 5=>95: 5(55.2 \mathrm{mg}, 0.246 \mathrm{mmol}$, colorless oil $)$.

${ }^{1} \mathrm{H}$ NMR (396 MHz, $\left.\mathrm{CDCl}_{3}, \delta\right): 0.89(\mathrm{t}, J=7.0 \mathrm{~Hz}, 3 \mathrm{H}), 1.06-1.20(\mathrm{~m}, 2 \mathrm{H}), 1.23-1.48(\mathrm{~m}, 12 \mathrm{H})$, 1.59 (s, 3H), 1.63-1.84 (m, 3H), 2.05-2.15 (m, 2H), 2.49-2.60 (m, 1H), 4.12 (s, 2H). ${ }^{13} \mathrm{C}$ NMR (100 $\left.\mathrm{MHz}, \mathrm{CDCl}_{3}, \delta\right): 13.5\left(\mathrm{CH}_{3}\right), 14.1\left(\mathrm{CH}_{3}\right), 22.6\left(\mathrm{CH}_{2}\right), 26.1\left(\mathrm{CH}_{2}\right), 26.5\left(\mathrm{CH}_{2}\right), 28.6\left(\mathrm{CH}_{2}\right), 31.4\left(\mathrm{CH}_{2}\right)$, $31.5\left(\mathrm{CH}_{2}\right), 32.0\left(\mathrm{CH}_{2}\right), 41.1(\mathrm{CH}), 61.6\left(\mathrm{CH}_{2}\right), 131.9(\mathrm{C}), 139.4(\mathrm{C})$. HRMS-EI $(\mathrm{m} / \mathrm{z})$ : $[\mathrm{M}]^{+}$calcd for $\mathrm{C}_{15} \mathrm{H}_{28} \mathrm{O}, 224.2140$; found, 224.2138 . 
(Z)-2-(1-Cyclohexylethylidene)-5-phenylpentan-1-ol [(Z)-6ad].

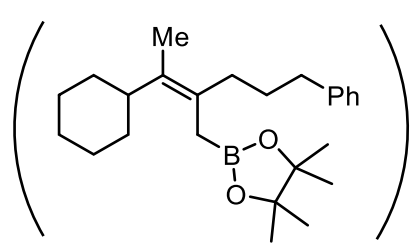

$(E)-\mathbf{4 a d}$<smiles>C[C@@H](/C(=C\CCc1ccccc1)CO)C1CCCCC1</smiles>

(Z)-6ad

The reaction was conducted with $68.1 \mathrm{mg}(0.500 \mathrm{mmol})$ of $\mathbf{1 a}$ according to the GP3. In order to remove an undesired boryl substitution product of the alkyl halide and protoboration product of the allene for the further purification and the determination of the regio- and stereoselectivities, the oxidation of the boryl groups was performed. The product (Z)-6ad was obtained in $63 \%$ yield with $E / Z=<5: 95,4: 5=>95: 5(86.3 \mathrm{mg}, 0.317 \mathrm{mmol}$, colorless oil).

${ }^{1} \mathrm{H}$ NMR $\left(392 \mathrm{MHz}, \mathrm{CDCl}_{3}, \delta\right): 1.06-1.47$ (m, 8H), 1.55 (s, 3H), 1.61-1.80 (m, 5H), 2.12-2.20 (m, $2 \mathrm{H}), 2.48-2.57(\mathrm{~m}, 1 \mathrm{H}), 2.58-2.66(\mathrm{~m}, 2 \mathrm{H}), 4.11(\mathrm{~s}, 2 \mathrm{H}), 7.12-7.20(\mathrm{~m}, 3 \mathrm{H}), 7.22-7.29(\mathrm{~m}, 2 \mathrm{H}) .{ }^{13} \mathrm{C}$ NMR (99 MHz, $\left.\mathrm{CDCl}_{3}, \delta\right)$ : $13.5\left(\mathrm{CH}_{3}\right), 26.1\left(\mathrm{CH}_{2}\right), 26.5\left(\mathrm{CH}_{2}\right), 30.7\left(\mathrm{CH}_{2}\right), 31.1\left(\mathrm{CH}_{2}\right), 31.5\left(\mathrm{CH}_{2}\right)$, $36.0\left(\mathrm{CH}_{2}\right), 41.1(\mathrm{CH}), 61.4\left(\mathrm{CH}_{2}\right), 125.6(\mathrm{CH}), 128.2(\mathrm{CH}), 128.3(\mathrm{CH}), 131.4(\mathrm{C}), 139.7(\mathrm{C}), 142.4$ (C). HRMS-EI $(\mathrm{m} / \mathrm{z})$ : [M] ${ }^{+}$calcd for $\mathrm{C}_{19} \mathrm{H}_{28} \mathrm{O}, 272.2140$; found, 272.2139 .

(E)-2-\{5-[4-(tert-Butyl)phenyl]-2-ethyl-3,4-dimethylpent-2-en-1-yl\}-4,4,5,5-tetramethyl-1,3,2dioxaborolane $[(E)-4 \mathrm{cb}]$.<smiles>CC/C(CB1OC(C)(C)C(C)(C)O1)=C(\C)C(C)Cc1ccc(C(C)(C)C)cc1</smiles>

$(E)-\mathbf{4 c b}$

The reaction was conducted with $114.0 \mathrm{mg}(0.499 \mathrm{mmol})$ of $1 \mathrm{c}$ according to the GP4. The product (E)-4cb was obtained in 88\% yield with $E / Z=88: 12,4: 5=>95: 5(169.3 \mathrm{mg}, 0.440 \mathrm{mmol}$, colorless oil).

${ }^{1} \mathrm{H}$ NMR $\left(392 \mathrm{MHz}, \mathrm{CDCl}_{3}, \delta\right): 0.83-0.94(\mathrm{~m}, 6 \mathrm{H}), 1.22(\mathrm{~s}, 12 \mathrm{H}), 1.30(\mathrm{~s}, 9 \mathrm{H}), 1.45-1.62(\mathrm{~m}, 5 \mathrm{H})$, $1.88-2.01(\mathrm{~m}, 1 \mathrm{H}), 2.02-2.14(\mathrm{~m}, 1 \mathrm{H}), 2.45(\mathrm{dd}, J=13.2,8.8 \mathrm{~Hz}, 1 \mathrm{H}), 2.60(\mathrm{dd}, J=13.0,6.3 \mathrm{~Hz}, 1 \mathrm{H})$, 2.79-2.88 (m, 1H), 7.06-7.14 (m, 2H), 7.22-7.28 (m, 2H). $\left.{ }^{13} \mathrm{C} \mathrm{NMR} \mathrm{(99} \mathrm{MHz,} \mathrm{CDCl}_{3}, \delta\right): 11.9\left(\mathrm{CH}_{3}\right)$, $12.2\left(\mathrm{CH}_{3}\right), 16.3\left(\mathrm{br}, \mathrm{B}-\mathrm{CH}_{2}\right), 17.7\left(\mathrm{CH}_{3}\right), 24.66\left(\mathrm{CH}_{3}\right), 24.74\left(\mathrm{CH}_{3}\right), 27.7\left(\mathrm{CH}_{2}\right), 31.4\left(\mathrm{CH}_{3}\right), 34.2(\mathrm{C})$, $37.8(\mathrm{CH}), 40.7\left(\mathrm{CH}_{2}\right), 82.8(\mathrm{C}), 124.6(\mathrm{CH}), 128.7(\mathrm{CH}), 129.6(\mathrm{C}), 129.9(\mathrm{C}), 138.6(\mathrm{C}), 148.0(\mathrm{C})$. ${ }^{11} \mathrm{~B}$ NMR (126 MHz, $\mathrm{CDCl}_{3}, \delta$ ): 32.7 (br, s). HRMS-ESI $(\mathrm{m} / \mathrm{z})$ : $[\mathrm{M}+\mathrm{Na}]^{+}$calcd for $\mathrm{C}_{25} \mathrm{H}_{41}{ }^{11} \mathrm{BO}_{2} \mathrm{Na}$, 407.3096; found, 407.3095. 
(E)-2-(2-\{4-[4-(tert-Butyl)phenyl]-3-methylbutan-2-ylidene $\}$ heptyl)-4,4,5,5-tetramethyl-1,3,2dioxaborolane $[(E)-4 \mathrm{cc}]$.

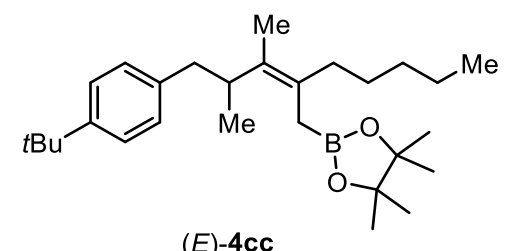

The reaction was conducted with $114.0 \mathrm{mg}(0.499 \mathrm{mmol})$ of $1 \mathrm{c}$ according to the GP4. The product (E)-4cc was obtained in 89\% yield with $E / Z=88: 12,4: 5=>95: 5(189.8 \mathrm{mg}, 0.445 \mathrm{mmol}$, colorless oil).

${ }^{1} \mathrm{H}$ NMR (392 MHz, $\left.\mathrm{CDCl}_{3}, \delta\right): 0.84-0.93$ (m, 6H), 1.16-1.33 (m, 6H), 1.22 (s, $\left.12 \mathrm{H}\right), 1.29(\mathrm{~s}, 9 \mathrm{H})$, $1.45-1.62(\mathrm{~m}, 5 \mathrm{H}), 1.86-1.97(\mathrm{~m}, 1 \mathrm{H}), 2.00-2.09(\mathrm{~m}, 1 \mathrm{H}), 2.46(\mathrm{dd}, J=13.5,8.5 \mathrm{~Hz}, 1 \mathrm{H}), 2.60(\mathrm{dd}$, $J=13.0,6.3 \mathrm{~Hz}, 1 \mathrm{H}), 2.81-2.90(\mathrm{~m}, 1 \mathrm{H}), 7.06-7.14(\mathrm{~m}, 2 \mathrm{H}), 7.22-7.29(\mathrm{~m}, 2 \mathrm{H}) .{ }^{13} \mathrm{C}$ NMR $(99 \mathrm{MHz}$, $\left.\mathrm{CDCl}_{3}, \delta\right): 12.1\left(\mathrm{CH}_{3}\right), 14.1\left(\mathrm{CH}_{3}\right), 16.8\left(\mathrm{br}, \mathrm{B}-\mathrm{CH}_{2}\right), 17.8\left(\mathrm{CH}_{3}\right), 22.7\left(\mathrm{CH}_{2}\right), 24.68\left(\mathrm{CH}_{3}\right), 24.74$ $\left(\mathrm{CH}_{3}\right), 27.6\left(\mathrm{CH}_{2}\right), 31.4\left(\mathrm{CH}_{3}\right), 31.9\left(\mathrm{CH}_{2}\right), 34.2(\mathrm{C}), 34.8\left(\mathrm{CH}_{2}\right), 37.8(\mathrm{CH}), 40.8\left(\mathrm{CH}_{2}\right), 82.8(\mathrm{C})$, 124.6 (CH), $128.6(C), 128.7(\mathrm{CH}), 130.1(C), 138.6(C), 148.0(C) .{ }^{11} \mathrm{~B} \mathrm{NMR}\left(126 \mathrm{MHz}, \mathrm{CDCl}_{3}, \delta\right)$ : 32.9 (br, s). HRMS-ESI $(\mathrm{m} / \mathrm{z})$ : $[\mathrm{M}+\mathrm{Na}]^{+}$calcd for $\mathrm{C}_{28} \mathrm{H}_{47}{ }^{11} \mathrm{BO}_{2} \mathrm{Na}, 449.3566$; found, 449.3565 .

(E)-2-(3-\{1-[4-(tert-Butyl)phenyl]propan-2-yl\{-2-ethylhex-2-en-1-yl)-4,4,5,5-tetramethyl-1,3,2dioxaborolane $[(E)-4 \mathrm{db}]$.

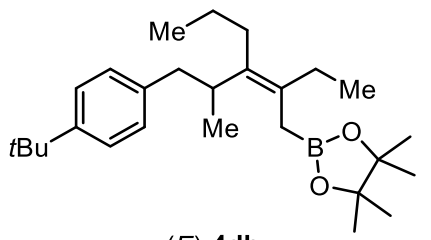

$(E)-\mathbf{4 d b}$

The reaction was conducted with $128.3 \mathrm{mg}(0.500 \mathrm{mmol})$ of $\mathbf{1 d}$ according to the GP4. The product (E)-4db was obtained in 87\% yield with $E / Z=89: 11, \mathbf{4 : 5}=>95: 5(179.9 \mathrm{mg}, 0.436 \mathrm{mmol}$, colorless oil).

${ }^{1} \mathrm{H}$ NMR (396 MHz, $\left.\mathrm{CDCl}_{3}, \delta\right): 0.82-0.97(\mathrm{~m}, 9 \mathrm{H}), 1.21(\mathrm{~s}, 12 \mathrm{H}), 1.30(\mathrm{~s}, 9 \mathrm{H}), 1.33-1.44(\mathrm{~m}, 2 \mathrm{H})$, $1.48-1.61(\mathrm{~m}, 2 \mathrm{H}), 1.85-2.11(\mathrm{~m}, 4 \mathrm{H}), 2.42(\mathrm{dd}, J=14.7,7.9 \mathrm{~Hz}, 1 \mathrm{H}), 2.65(\mathrm{dd}, J=12.9,5.7 \mathrm{~Hz}, 1 \mathrm{H})$, 2.80-2.90 (m, 1H), 7.08-7.15 (m, 2H), 7.21-7.29 (m, 2H). ${ }^{13} \mathrm{C} \mathrm{NMR}\left(100 \mathrm{MHz}, \mathrm{CDCl}_{3}, \delta\right): 12.9\left(\mathrm{CH}_{3}\right)$, $14.9\left(\mathrm{CH}_{3}\right), 15.6\left(\mathrm{br}, \mathrm{B}-\mathrm{CH}_{2}\right), 18.0\left(\mathrm{CH}_{3}\right), 24.66\left(\mathrm{CH}_{3}\right.$ and $\left.\mathrm{CH}_{2}\right), 24.70\left(\mathrm{CH}_{3}\right), 27.1\left(\mathrm{CH}_{2}\right), 30.2\left(\mathrm{CH}_{2}\right)$, $31.4\left(\mathrm{CH}_{3}\right), 34.2(\mathrm{C}), 38.2(\mathrm{CH}), 41.4\left(\mathrm{CH}_{2}\right), 82.7(\mathrm{C}), 124.6(\mathrm{CH}), 128.9(\mathrm{CH}), 130.9(C), 135.0(C)$, $138.7(C), 148.0(C) .{ }^{11} \mathrm{~B}$ NMR (126 MHz, $\left.\mathrm{CDCl}_{3}, \delta\right): 32.7$ (br, s). HRMS-ESI $(\mathrm{m} / \mathrm{z})$ : $[\mathrm{M}+\mathrm{Na}]^{+}$calcd for $\mathrm{C}_{27} \mathrm{H}_{45}{ }^{11} \mathrm{BO}_{2} \mathrm{Na}$, 435.3410; found, 435.3409 . 
(E)-2-(2-\{1-[4-(tert-Butyl)phenyl]-2-methylhexan-3-ylidene $\}$ heptyl)-4,4,5,5-tetramethyl-1,3,2dioxaborolane $[(E)-4 d c]$.

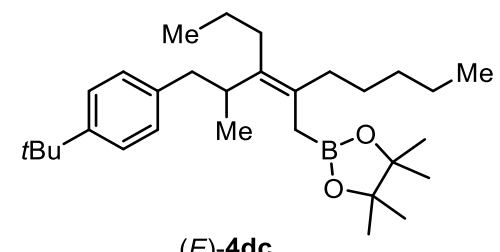

$(E)-4 d c$

The reaction was conducted with $128.4 \mathrm{mg}(0.501 \mathrm{mmol})$ of $\mathbf{1 d}$ according to the GP4. The product (E)-4dc was obtained in 87\% yield with $E / Z=89: 11, \mathbf{4 : 5}=>95: 5(197.4 \mathrm{mg}, 0.434 \mathrm{mmol}$, colorless oil).

${ }^{1} \mathrm{H}$ NMR (396 MHz, $\left.\mathrm{CDCl}_{3}, \delta\right): 0.85-0.96$ (m, 9H), 1.17-1.43 (m, 8H), 1.21 (s, 9H), $1.30(\mathrm{~s}, 12 \mathrm{H})$, $1.47-1.62(\mathrm{~m}, 2 \mathrm{H}), 1.84-2.08(\mathrm{~m}, 4 \mathrm{H}), 2.42(\mathrm{dd}, J=12.9,9.3 \mathrm{~Hz}, 1 \mathrm{H}), 2.65(\mathrm{dd}, J=13.1,5.4 \mathrm{~Hz}, 1 \mathrm{H})$, 2.80-2.94 (m, 1H), 7.07-7.15 (m, 2H), 7.22-7.31 (m, 2H). ${ }^{13} \mathrm{C} \mathrm{NMR}\left(100 \mathrm{MHz}, \mathrm{CDCl}_{3}, \delta\right): 14.1\left(\mathrm{CH}_{3}\right)$, $14.9\left(\mathrm{CH}_{3}\right), 16.2$ (br, B-CH$), 18.1\left(\mathrm{CH}_{3}\right), 22.7\left(\mathrm{CH}_{2}\right), 24.6\left(\mathrm{CH}_{2}\right), 24.68\left(\mathrm{CH}_{3}\right), 24.70\left(\mathrm{CH}_{3}\right), 28.1$ $\left(\mathrm{CH}_{2}\right), 30.3\left(\mathrm{CH}_{2}\right), 31.4\left(\mathrm{CH}_{3}\right), 32.2\left(\mathrm{CH}_{2}\right), 34.2(\mathrm{C}), 34.4\left(\mathrm{CH}_{2}\right), 38.2(\mathrm{CH}), 41.4\left(\mathrm{CH}_{2}\right), 82.7(\mathrm{C})$, 124.6 (CH), $128.9(\mathrm{CH}), 129.8(C), 135.4(C), 138.7(C), 148.0(C) .{ }^{11} \mathrm{~B} \mathrm{NMR}\left(126 \mathrm{MHz}, \mathrm{CDCl}_{3}, \delta\right)$ : 33.3 (br, s). HRMS-ESI $(\mathrm{m} / \mathrm{z})$ : $[\mathrm{M}+\mathrm{Na}]^{+}$calcd for $\mathrm{C}_{30} \mathrm{H}_{51}{ }^{11} \mathrm{BO}_{2} \mathrm{Na}$, 477.3880; found, 477.3877 .

\section{(E)-2-(3-\{1-[4-(tert-Butyl)phenyl]propan-2-yl\}-2-ethylundec-2-en-1-yl)-4,4,5,5-tetramethyl-}

\section{1,3,2-dioxaborolane $[(E)-4 \mathrm{eb}]$.}

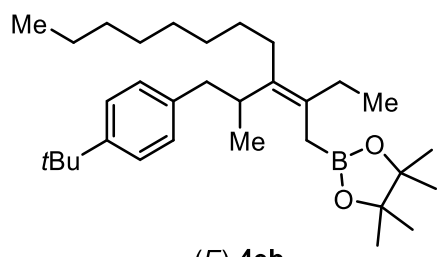

(E)-4eb

The reaction was conducted with $212.0 \mathrm{mg}(0.497 \mathrm{mmol})$ of $\mathbf{1 e}$ according to the GP4. The product (E)-4eb was obtained in 82\% yield with $E / Z=89: 11,4: 5=>95: 5(197.5 \mathrm{mg}, 0.409 \mathrm{mmol}$, colorless oil).

${ }^{1} \mathrm{H}$ NMR (396 MHz, $\left.\mathrm{CDCl}_{3}, \delta\right): 0.82-0.96(\mathrm{~m}, 9 \mathrm{H}), 1.16-1.41(\mathrm{~m}, 12 \mathrm{H}), 1.21(\mathrm{~s}, 12 \mathrm{H}), 1.30$ (s, 9H), 1.49-1.60 (m, 2H), 1.85-2.12 (m, 4H), 2.42 (dd, $J=13.1,9.5 \mathrm{~Hz}, 1 \mathrm{H}), 2.65$ (dd, $J=12.9,5.7$ $\mathrm{Hz}, 1 \mathrm{H}), 2.79-2.92(\mathrm{~m}, 1 \mathrm{H}), 7.08-7.15(\mathrm{~m}, 2 \mathrm{H}), 7.23-7.29$ (m, 2H). ${ }^{13} \mathrm{C} \mathrm{NMR}\left(100 \mathrm{MHz}, \mathrm{CDCl}_{3}, \delta\right)$ : $12.9\left(\mathrm{CH}_{3}\right), 14.1\left(\mathrm{CH}_{3}\right), 15.7\left(\mathrm{br}, \mathrm{B}-\mathrm{CH}_{2}\right), 18.0\left(\mathrm{CH}_{3}\right), 22.7\left(\mathrm{CH}_{2}\right), 24.67\left(\mathrm{CH}_{3}\right.$ and $\left.\mathrm{CH}_{2}\right), 24.70\left(\mathrm{CH}_{3}\right)$, $27.1\left(\mathrm{CH}_{2}\right), 27.9\left(\mathrm{CH}_{2}\right), 29.4\left(\mathrm{CH}_{2}\right), 29.5\left(\mathrm{CH}_{2}\right), 30.6\left(\mathrm{CH}_{2}\right), 31.5\left(\mathrm{CH}_{3}\right), 31.9\left(\mathrm{CH}_{2}\right), 34.2(C), 38.2$ $(\mathrm{CH}), 41.4\left(\mathrm{CH}_{2}\right), 82.7(\mathrm{C}), 124.6(\mathrm{CH}), 128.9(\mathrm{CH}), 130.8(C), 135.1(\mathrm{C}), 138.7(C), 148.0(C) .{ }^{11} \mathrm{~B}$ NMR (126 MHz, $\left.\mathrm{CDCl}_{3}, \delta\right): 32.6$ (br, s). HRMS-EI $(\mathrm{m} / \mathrm{z})$ : $[\mathrm{M}-\mathrm{Me}]^{+}$calcd for $\mathrm{C}_{31} \mathrm{H}_{52}{ }^{11} \mathrm{BO}_{2}, 467.4066$; found, 467.4063 . 
(Z)-3-\{1-[4-(tert-Butyl)phenyl]propan-2-yl\}-2-pentylundec-2-en-1-ol [(Z)-6ec].

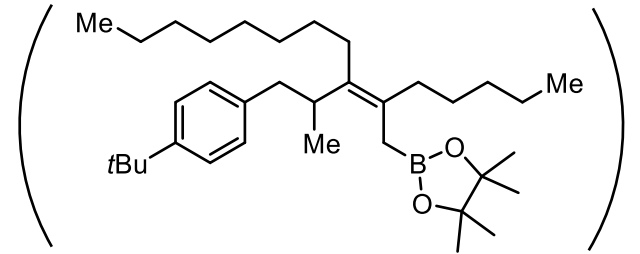

(E)-4ec

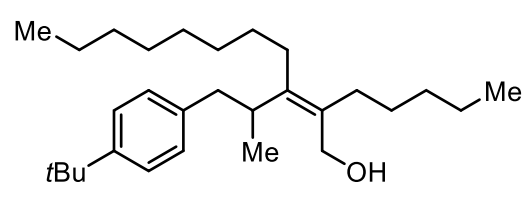

(Z)-6ec

The reaction was conducted with $213.2 \mathrm{mg}(0.500 \mathrm{mmol})$ of $1 \mathbf{e}$ according to the GP4. In order to remove an undesired boryl substitution product of the alkyl halide and protoboration product of the allene for the further purification and the determination of the regio- and stereoselectivities, the oxidation of the boryl groups was performed. The corresponding alcohol (Z)-6ec was obtained in $50 \%$ yield with $E / Z=87: 13,4: 5=>95: 5(103.8 \mathrm{mg}, 0.250 \mathrm{mmol}$, colorless oil $)$.

${ }^{1} \mathrm{H}$ NMR $\left(392 \mathrm{MHz}, \mathrm{CDCl}_{3}, \delta\right):-0.13(\mathrm{dd}, J=8.3,3.8 \mathrm{~Hz}, 1 \mathrm{H}), 0.83-0.98(\mathrm{~m}, 6 \mathrm{H}), 1.07$ (d, $J=$ $7.2 \mathrm{~Hz}, 3 \mathrm{H}), 1.17-1.46(\mathrm{~m}, 18 \mathrm{H}), 1.28(\mathrm{~s}, 9 \mathrm{H}), 1.86-2.15$ (m, 4H), 2.47-2.64 (m, 2H), 2.85-3.03 (m, $1 \mathrm{H}), 3.66(\mathrm{dd}, J=11.7,8.5 \mathrm{~Hz}, 1 \mathrm{H}), 3.85(\mathrm{dd}, J=11.4,3.8 \mathrm{~Hz}, 1 \mathrm{H}), 6.98-7.08$ (m, 2H), 7.24-7.31 (m, 2H). ${ }^{13} \mathrm{C} \mathrm{NMR}\left(99 \mathrm{MHz}, \mathrm{CDCl}_{3}, \delta\right): 14.06\left(\mathrm{CH}_{3}\right), 14.11\left(\mathrm{CH}_{3}\right), 19.8\left(\mathrm{CH}_{3}\right), 22.6\left(\mathrm{CH}_{2}\right), 22.7\left(\mathrm{CH}_{2}\right)$, $28.0\left(\mathrm{CH}_{2}\right), 28.6\left(\mathrm{CH}_{2}\right), 29.31\left(\mathrm{CH}_{2}\right), 29.33\left(\mathrm{CH}_{2}\right), 30.6\left(\mathrm{CH}_{2}\right), 31.0\left(\mathrm{CH}_{2}\right), 31.3\left(\mathrm{CH}_{3}\right), 31.4\left(\mathrm{CH}_{2}\right)$, $31.9\left(\mathrm{CH}_{2}\right), 32.3\left(\mathrm{CH}_{2}\right), 34.3(\mathrm{C}), 38.7(\mathrm{CH}), 41.7\left(\mathrm{CH}_{2}\right), 61.1\left(\mathrm{CH}_{2}\right), 124.9(\mathrm{CH}), 128.8(\mathrm{CH}), 134.7$ (C), $138.2(C), 140.3(C), 148.8(C)$. HRMS-EI $(m / z)$ : $[\mathrm{M}]^{+}$calcd for $\mathrm{C}_{29} \mathrm{H}_{50} \mathrm{O}, 414.3862$; found, 414.3853 .

(E)-2-(3-\{1-[4-(tert-Butyl)phenyl]propan-2-yl\}-2-ethyl-5-methylhex-2-en-1-yl)-4,4,5,5tetramethyl-1,3,2-dioxaborolane $[(E)-4 \mathrm{fb}]$.

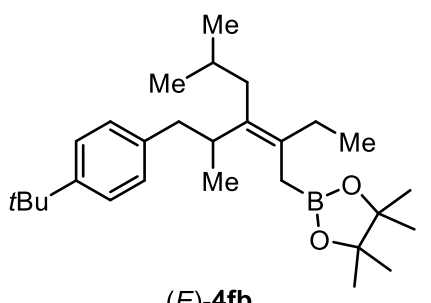

(E)-4fb

The reaction was conducted with $135.3 \mathrm{mg}(0.500 \mathrm{mmol})$ of $\mathbf{1 f}$ according to the GP4. The product (E)-4fb was obtained in 79\% yield with $E / Z=89: 11, \mathbf{4 : 5}=>95: 5$ (169.6 mg, $0.398 \mathrm{mmol}$, colorless oil).

${ }^{1} \mathrm{H}$ NMR (392 MHz, $\left.\mathrm{CDCl}_{3}, \delta\right): 0.85-0.98$ (m, 12H), 1.22 (s, 12H), 1.30 (s, 9H), 1.63-1.72 (m, 2H), 1.76-1.88 (m, 1H), 1.89-2.19 (m, 4H), 2.44 (dd, $J=13.0,9.9 \mathrm{~Hz}, 1 \mathrm{H}), 2.72(\mathrm{dd}, J=13.0,5.4$ $\mathrm{Hz}, 1 \mathrm{H}), 2.76-2.88$ (m, 1H), 7.10-7.15 (m, 2H), 7.23-7.30 (m, 2H). ${ }^{13} \mathrm{C} \mathrm{NMR} \mathrm{(99} \mathrm{MHz,} \mathrm{CDCl}_{3}, \delta$ ): $12.6\left(\mathrm{CH}_{3}\right), 16.3\left(\mathrm{br}, \mathrm{B}-\mathrm{CH}_{2}\right), 18.3\left(\mathrm{CH}_{3}\right), 22.7\left(\mathrm{CH}_{3}\right), 22.8\left(\mathrm{CH}_{3}\right), 24.7\left(\mathrm{CH}_{3}\right), 24.8\left(\mathrm{CH}_{3}\right), 27.8\left(\mathrm{CH}_{2}\right)$, $28.6(\mathrm{CH}), 31.4\left(\mathrm{CH}_{3}\right), 34.2(\mathrm{C}), 37.3\left(\mathrm{CH}_{2}\right), 38.5(\mathrm{CH}), 41.7\left(\mathrm{CH}_{2}\right), 82.8(\mathrm{C}), 124.7(\mathrm{CH}), 128.8(\mathrm{CH})$, $132.6(C), 133.2(C), 138.9(C), 148.0(C) .{ }^{11} \mathrm{~B}$ NMR (126 MHz, $\left.\mathrm{CDCl}_{3}, \delta\right): 32.9$ (br, s). HRMS-ESI $(m / z):[\mathrm{M}+\mathrm{Na}]^{+}$calcd for $\mathrm{C}_{28} \mathrm{H}_{47}{ }^{11} \mathrm{BO}_{2} \mathrm{Na}, 449.3566$; found, 449.3565 . 


\section{1,3,2-dioxaborolane $[(E)-4 f c]$.}<smiles>CCCCCC(CC(C)C)=C(Cc1ccc(Br)cc1)CC(C)C</smiles>

$(E)-\mathbf{4 f c}$

The reaction was conducted with $135.0 \mathrm{mg}(0.499 \mathrm{mmol})$ of $\mathbf{1 f}$ according to the GP4. The product (E)-4fe was obtained in 76\% yield with $E / Z=91: 9,4: 5=>95: 5(177.4 \mathrm{mg}, 0.379 \mathrm{mmol}$, colorless oil).

${ }^{1} \mathrm{H}$ NMR (392 MHz, $\mathrm{CDCl}_{3}, \delta$ ): 0.83-0.95 (m, 12H), 1.14-1.36 (m, 6H), $1.22(\mathrm{~s}, 12 \mathrm{H}), 1.30$ (s, 9H), 1.63-1.73 (m, 2H), 1.76-1.86 (m, 1H), 1.89-2.15 (m, 4H), 2.44 (dd, $J=13.2,9.6 \mathrm{~Hz}, 1 \mathrm{H}), 2.73$ (dd, $J=13.2,5.2 \mathrm{~Hz}, 1 \mathrm{H}), 2.77-2.88(\mathrm{~m}, 1 \mathrm{H}), 7.09-7.15(\mathrm{~m}, 2 \mathrm{H}), 7.23-7.28(\mathrm{~m}, 2 \mathrm{H}) .{ }^{13} \mathrm{C}$ NMR $(99$ $\left.\mathrm{MHz}, \mathrm{CDCl}_{3}, \delta\right): 14.1\left(\mathrm{CH}_{3}\right), 16.8$ (br, B-CH$), 18.4\left(\mathrm{CH}_{3}\right), 22.6\left(\mathrm{CH}_{3}\right), 22.7\left(\mathrm{CH}_{2}\right), 22.8\left(\mathrm{CH}_{3}\right), 24.7$ $\left(\mathrm{CH}_{3}\right), 24.8\left(\mathrm{CH}_{3}\right), 27.9\left(\mathrm{CH}_{2}\right), 28.7(\mathrm{CH}), 31.4\left(\mathrm{CH}_{3}\right), 32.2\left(\mathrm{CH}_{2}\right), 34.2(\mathrm{C}), 35.1\left(\mathrm{CH}_{2}\right), 37.4\left(\mathrm{CH}_{2}\right)$, $38.5(\mathrm{CH}), 41.7\left(\mathrm{CH}_{2}\right), 82.8(\mathrm{C}), 124.7(\mathrm{CH}), 128.8(\mathrm{CH}), 131.4(\mathrm{C}), 133.6(\mathrm{C}), 138.9(\mathrm{C}), 148.0(\mathrm{C})$. ${ }^{11} \mathrm{~B}$ NMR (126 MHz, $\mathrm{CDCl}_{3}, \delta$ ): 33.7 (br, s). HRMS-ESI $(\mathrm{m} / \mathrm{z})$ : $[\mathrm{M}+\mathrm{Na}]^{+}$calcd for $\mathrm{C}_{31} \mathrm{H}_{53}{ }^{11} \mathrm{BO}_{2} \mathrm{Na}$, 491.4036; found, 491.4036.

\section{2-\{(E)-2-[1-(Adamantan-1-yl)ethylidene]-6,6,6-trifluorohexyl\}-4,4,5,5-tetramethyl-1,3,2-} dioxaborolane $[(E)-4 \mathrm{ga}]$.

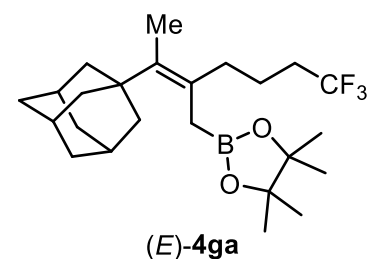

The reaction was conducted with $94.1 \mathrm{mg}(0.500 \mathrm{mmol})$ of $1 \mathrm{~g}$ according to the GP4. Then, the mixture of the product and byproducts was exposed to $\mathrm{NaBO}_{3} \cdot 4 \mathrm{H}_{2} \mathrm{O}(300 \mathrm{mg}, 1.94 \mathrm{mmol})$ in THF $(2.0 \mathrm{~mL})$ and $\mathrm{H}_{2} \mathrm{O}(2.0 \mathrm{~mL})$ at room temperature for $4 \mathrm{~h}$. (E)-4ga was not oxidized under the conditions, and the pure product $(E)$-4ga was obtained in 64\% yield with $E / Z=>95: 5, \mathbf{4 : 5}=>95: 5(135.6 \mathrm{mg}$, $0.318 \mathrm{mmol}$, white solid).

${ }^{1} \mathrm{H}$ NMR $\left(396 \mathrm{MHz}, \mathrm{CDCl}_{3}, \delta\right): 1.23$ (s, 12H), 1.55-1.73 (m, 11H), 1.83-1.98 (m, 11H), 2.00-2.16 (m, 4H). ${ }^{13} \mathrm{C} \mathrm{NMR}\left(100 \mathrm{MHz}, \mathrm{CDCl}_{3}, \delta\right): 16.0\left(\mathrm{CH}_{3}\right), 20.2\left(\mathrm{~B}-\mathrm{CH}_{2}\right.$ and $\left.\mathrm{CH}_{2}\right), 24.6\left(\mathrm{CH}_{3}\right), 29.2(\mathrm{CH})$, 33.6 (q, $\left.J=28.4 \mathrm{~Hz}, \mathrm{CH}_{2}\right), 37.0\left(\mathrm{CH}_{2}\right), 37.3\left(\mathrm{CH}_{2}\right), 38.5(C), 41.0\left(\mathrm{CH}_{2}\right), 83.0(C), 127.3(\mathrm{q}, J=277.5$ $\mathrm{Hz}, C), 128.1(C), 135.1(C) .{ }^{11} \mathrm{~B}$ NMR $\left(126 \mathrm{MHz}, \mathrm{CDCl}_{3}, \delta\right): 32.9$ (br, s). HRMS-EI $(\mathrm{m} / \mathrm{z}):[\mathrm{M}]^{+} \mathrm{calcd}$ for $\mathrm{C}_{24} \mathrm{H}_{38}{ }^{11} \mathrm{BF}_{3} \mathrm{O}_{2}, 426.2921$; found, 426.2931 . 
(E)-2-[3-(tert-Butyl)-2-(4,4,4-trifluorobutyl)hept-2-en-1-yl]-4,4,5,5-tetramethyl-1,3,2dioxaborolane $[(E)-4 h a]$.

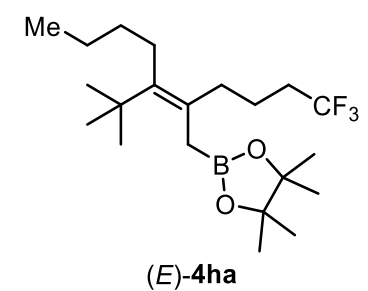

The reaction was conducted with $76.1 \mathrm{mg}(0.500 \mathrm{mmol})$ of $\mathbf{1 h}$ according to the GP4. The product (E)-4ha was obtained in 69\% yield with $E / Z=>95: 5, \mathbf{4 : 5}=>95: 5(134.1 \mathrm{mg}, 0.344 \mathrm{mmol}$, colorless oil).

${ }^{1} \mathrm{H} \mathrm{NMR}\left(396 \mathrm{MHz}, \mathrm{CDCl}_{3}, \delta\right): 0.91(\mathrm{t}, J=7.1 \mathrm{~Hz}, 3 \mathrm{H}), 1.16(\mathrm{~s}, 9 \mathrm{H}), 1.22(\mathrm{~s}, 12 \mathrm{H}), 1.27-1.39(\mathrm{~m}$, $4 \mathrm{H}), 1.60-1.71(\mathrm{~m}, 2 \mathrm{H}), 1.85(\mathrm{~s}, 2 \mathrm{H}), 1.96-2.14(\mathrm{~m}, 6 \mathrm{H}) .{ }^{13} \mathrm{C} \mathrm{NMR}\left(100 \mathrm{MHz}, \mathrm{CDCl}_{3}, \delta\right): 13.9\left(\mathrm{CH}_{3}\right)$, 19.6 (br, B-CH $\left.\mathrm{CH}_{2}\right), 21.0\left(\mathrm{CH}_{2}\right), 23.3\left(\mathrm{CH}_{2}\right), 24.6\left(\mathrm{CH}_{3}\right), 31.0\left(\mathrm{CH}_{2}\right), 31.4\left(\mathrm{CH}_{3}\right), 33.7\left(\mathrm{CH}_{2}\right), 33.8$ (q, $J$ $\left.=28.4 \mathrm{~Hz}, \mathrm{CH}_{2}\right), 35.5\left(\mathrm{CH}_{2}\right), 35.7(C), 83.0(C), 127.3$ (q, $\left.J=277.5 \mathrm{~Hz}, C\right), 129.1(C), 140.1(C) .{ }^{11} \mathrm{~B}$ NMR (126 MHz, $\mathrm{CDCl}_{3}, \delta$ ): 33.0 (br, s). HRMS-EI $(\mathrm{m} / \mathrm{z})$ : $[\mathrm{M}]^{+}$calcd for $\mathrm{C}_{21} \mathrm{H}_{38}{ }^{11} \mathrm{BF}_{3} \mathrm{O}_{2}, 390.2921$; found, 390.2911 .

(E)-2-[3-(tert-Butyl)-2-pentylhept-2-en-1-yl]-4,4,5,5-tetramethyl-1,3,2-dioxaborolane [(E)-4hc].<smiles>CCCCCC(CCCC)=C(CCCC)C(C)(C)C</smiles>

$(E)-4 h c$

The reaction was conducted with $76.1 \mathrm{mg}(0.500 \mathrm{mmol})$ of $\mathbf{1 h}$ according to the GP4. The product (E)-4he was obtained in 92\% yield with $E / Z=>95: 5,4: 5=>95: 5(160.8 \mathrm{mg}, 0.459 \mathrm{mmol}$, colorless oil).

${ }^{1} \mathrm{H}$ NMR (392 MHz, $\mathrm{CDCl}_{3}, \delta$ ): 0.83-0.96 (m, 6H), 1.09-1.44 (m, 10H), 1.16 (s, 9H), $1.22(\mathrm{~s}, 12 \mathrm{H})$, $1.86(\mathrm{~s}, 2 \mathrm{H}), 1.92-2.08(\mathrm{~m}, 4 \mathrm{H}) .{ }^{13} \mathrm{C} \mathrm{NMR}\left(99 \mathrm{MHz}, \mathrm{CDCl}_{3}, \delta\right): 14.0\left(\mathrm{CH}_{3}\right), 14.1\left(\mathrm{CH}_{3}\right), 19.7$ (br, B$\left.\mathrm{CH}_{2}\right), 22.7\left(\mathrm{CH}_{2}\right), 23.3\left(\mathrm{CH}_{2}\right), 24.7\left(\mathrm{CH}_{3}\right), 28.6\left(\mathrm{CH}_{2}\right), 30.9\left(\mathrm{CH}_{2}\right), 31.4\left(\mathrm{CH}_{3}\right), 32.5\left(\mathrm{CH}_{2}\right), 33.9\left(\mathrm{CH}_{2}\right)$, $35.4(C), 36.8\left(\mathrm{CH}_{2}\right), 82.8(C), 130.9(C), 138.4(C) .{ }^{11} \mathrm{~B}$ NMR (126 MHz, $\left.\mathrm{CDCl}_{3}, \delta\right): 33.1$ (br, s). HRMS-EI $(m / z)$ : [M] $]^{+}$calcd for $\mathrm{C}_{22} \mathrm{H}_{43}{ }^{11} \mathrm{BO}_{2}, 350.3360$; found, 350.3358 . 
(Z)-3-Methyl-6-phenyl-2-(4,4,4-trifluorobutyl)hex-2-en-1-ol [(Z)-6ia].<smiles>CCCCC/C(C)=C(/CCCC)C[BH-]1OC(C)(C)C(C)(C)O1</smiles>

$(E)-4$ ia<smiles>CC(CCCc1ccccc1)=C(CO)CCCC(F)(F)F</smiles>

(Z)-6ia

The reaction was conducted with $86.1 \mathrm{mg}(0.500 \mathrm{mmol})$ of $\mathbf{1} \mathbf{i}$ according to the GP3. In order to remove an undesired boryl substitution product of the alkyl halide and protoboration product of the allene for the further purification and the determination of the regio- and stereoselectivities, the oxidation of the boryl groups was performed. The product (Z)-6ia was obtained in $59 \%$ yield with $E / Z$ $=11: 89,4: 5=>95: 5(87.9 \mathrm{mg}, 0.293 \mathrm{mmol}$, colorless oil $)$.

${ }^{1} \mathrm{H}$ NMR $\left(396 \mathrm{MHz}, \mathrm{CDCl}_{3}, \delta\right): 1.18-1.35$ (br-m, 1H), 1.52-1.81 (m, 7H), 1.91-2.32 (m, 6H), 2.59 (t, $J=7.7 \mathrm{~Hz}, 2 \mathrm{H}), 4.05$ (s, 2H), 7.12-7.22 (m, 3H), 7.24-7.32 (m, 2H). ${ }^{13} \mathrm{C} \mathrm{NMR}\left(100 \mathrm{MHz}, \mathrm{CDCl}_{3}\right.$,

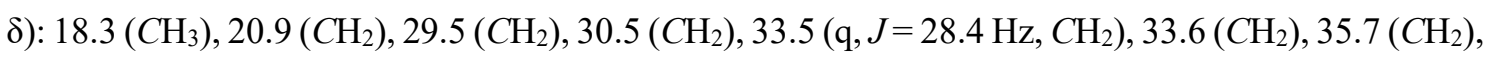
$61.3\left(\mathrm{CH}_{2}\right), 125.8(\mathrm{CH}), 128.26(\mathrm{CH}), 128.28(\mathrm{CH}), 129.5(\mathrm{q}, J=274.7 \mathrm{~Hz}, C), 131.5(C), 135.2(C)$, $142.1(C)$. HRMS-EI $(m / z)$ : $[\mathrm{M}]^{+}$calcd for $\mathrm{C}_{17} \mathrm{H}_{23} \mathrm{~F}_{3} \mathrm{O}, 300.1701$; found, 300.1702 .

(Z)-2-(5-Phenylpentan-2-ylidene)heptan-1-ol [(Z)-6ic].<smiles>CCCCCC(CB1OC(C)(C)C(C)(C)O1)=C(C)CCCc1ccccc1</smiles>

$(E)-4$ ic<smiles>CCCCCC(CO)=C(C)CCCc1ccccc1</smiles>

(Z)-6ic

The reaction was conducted with $86.2 \mathrm{mg}(0.500 \mathrm{mmol})$ of $1 \mathbf{i}$ according to the GP3. In order to remove an undesired boryl substitution product of the alkyl halide and protoboration product of the allene for the further purification and the determination of the regio- and stereoselectivities, the oxidation of the boryl groups was performed. The product (Z)-6ic was obtained in $52 \%$ yield with $E / Z$ $=10: 90,4: 5=>95: 5(67.5 \mathrm{mg}, 0.259 \mathrm{mmol}$, colorless oil $)$.

${ }^{1} \mathrm{H}$ NMR $\left(396 \mathrm{MHz}, \mathrm{CDCl}_{3}, \delta\right): 0.89(\mathrm{t}, J=6.9 \mathrm{~Hz}, 3 \mathrm{H}), 1.13-1.45(\mathrm{~m}, 7 \mathrm{H}), 1.61-1.78(\mathrm{~m}, 2 \mathrm{H})$, 1.69 (s, 3H), 2.01-2.23 (m, 4H), $2.58(\mathrm{t}, J=7.7 \mathrm{~Hz}, 2 \mathrm{H}), 4.05$ (s, 2H), 7.12-7.21 (m, 3H), 7.22-7.32 (m, 2H). ${ }^{13} \mathrm{C} \mathrm{NMR}\left(100 \mathrm{MHz}, \mathrm{CDCl}_{3}, \delta\right): 14.1\left(\mathrm{CH}_{3}\right), 18.2\left(\mathrm{CH}_{3}\right), 22.6\left(\mathrm{CH}_{2}\right), 28.5\left(\mathrm{CH}_{2}\right), 30.6\left(\mathrm{CH}_{2}\right)$, $30.7\left(\mathrm{CH}_{2}\right), 32.0\left(\mathrm{CH}_{2}\right), 33.7\left(\mathrm{CH}_{2}\right), 35.7\left(\mathrm{CH}_{2}\right), 61.7\left(\mathrm{CH}_{2}\right), 125.7(\mathrm{CH}), 128.2(\mathrm{CH}), 133.3(\mathrm{C}), 133.8$ (C), $142.3(C)$. HRMS-EI $(m / z)$ : [M] calcd for $\mathrm{C}_{18} \mathrm{H}_{28} \mathrm{O}, 260.2140$; found, 260.2141 . 
(Z)-3-Methyl-2-(4,4,4-trifluorobutyl)undec-2-en-1-ol [(Z)-6ja].<smiles>CCCCCCCC/C(C)=C(\CCCC(F)(F)F)CB1OC(C)(C)C(C)(C)O1</smiles>

$(E)-4 \mathbf{j a}$<smiles>CCCCCCCC/C(C)=C(\CO)CCCC(F)(F)F</smiles>

(Z)-6ja

The reaction was conducted with $83.1 \mathrm{mg}(0.500 \mathrm{mmol})$ of $\mathbf{1 j}$ according to the GP3. In order to remove an undesired boryl substitution product of the alkyl halide and protoboration product of the allene for the further purification and the determination of the regio- and stereoselectivities, the oxidation of the boryl groups was performed. The product (Z)-6ja was obtained in $64 \%$ yield with $E / Z$ $=8: 92,4: 5=>95: 5(94.7 \mathrm{mg}, 0.322 \mathrm{mmol}$, colorless oil $)$.

${ }^{1} \mathrm{H}$ NMR $\left(392 \mathrm{MHz}, \mathrm{CDCl}_{3}, \delta\right): 0.88(\mathrm{t}, J=6.7 \mathrm{~Hz}, 3 \mathrm{H}), 1.13-1.50(\mathrm{~m}, 13 \mathrm{H}), 1.56-1.78(\mathrm{~m}, 5 \mathrm{H})$, 1.97-2.16 (m, 4H), $2.22(\mathrm{t}, J=8.0 \mathrm{~Hz}, 2 \mathrm{H}), 4.12(\mathrm{~s}, 2 \mathrm{H}) .{ }^{13} \mathrm{C} \mathrm{NMR}\left(99 \mathrm{MHz}, \mathrm{CDCl}_{3}, \delta\right): 14.0\left(\mathrm{CH}_{3}\right)$, $18.4\left(\mathrm{CH}_{3}\right), 21.0\left(\mathrm{CH}_{2}\right), 22.6\left(\mathrm{CH}_{2}\right), 29.0\left(\mathrm{CH}_{2}\right), 29.2\left(\mathrm{CH}_{2}\right), 29.5\left(\mathrm{CH}_{2}\right), 29.6\left(\mathrm{CH}_{2}\right), 31.8\left(\mathrm{CH}_{2}\right), 33.5$ (q, $\left.J=28.6 \mathrm{~Hz}, \mathrm{CH}_{2}\right), 34.1\left(\mathrm{CH}_{2}\right), 61.4\left(\mathrm{CH}_{2}\right), 127.2$ (q, $\left.J=277.6 \mathrm{~Hz}, C\right), 131.1(C), 135.9(C)$. HRMS-EI $(m / z):[M]^{+}$calcd for $\mathrm{C}_{16} \mathrm{H}_{29} \mathrm{~F}_{3} \mathrm{O}, 294.2171$; found, 294.2168 .

\section{(Z)-3-Methyl-2-pentylundec-2-en-1-ol [(Z)-6jc].}<smiles>CCCCCCCC/C(C)=C(\CCCCC)CB1OC(C)(C)C(C)(C)O1</smiles>

$(E)-4 \mathrm{jc}$

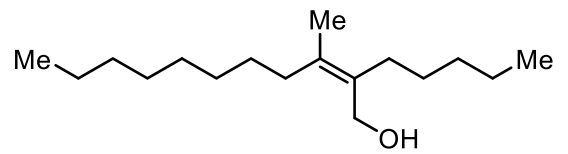

(Z)-6jc

The reaction was conducted with $83.3 \mathrm{mg}(0.501 \mathrm{mmol})$ of $\mathbf{1} \mathbf{j}$ according to the GP3. In order to remove an undesired boryl substitution product of the alkyl halide and protoboration product of the allene for the further purification and the determination of the regio- and stereoselectivities, the oxidation of the boryl groups was performed. The product (Z)-6je was obtained in $46 \%$ yield with $E / Z$ $=6: 94,4: 5=>95: 5(58.9 \mathrm{mg}, 0.231 \mathrm{mmol}$, colorless oil $)$.

${ }^{1} \mathrm{H}$ NMR (392 MHz, $\mathrm{CDCl}_{3}, \delta$ ): 0.79-0.98 (m, 6H), 1.11-1.50 (m, 19H), 1.68 (s, 3H), 1.99-2.19 (m, 4H), 4.10 (s, 2H). ${ }^{13} \mathrm{C}$ NMR (99 MHz, $\left.\mathrm{CDCl}_{3}, \delta\right): 14.1\left(\mathrm{CH}_{3}\right), 18.2\left(\mathrm{CH}_{3}\right), 22.59\left(\mathrm{CH}_{2}\right), 22.63$ $\left(\mathrm{CH}_{2}\right), 28.6\left(\mathrm{CH}_{2}\right), 29.0\left(\mathrm{CH}_{2}\right), 29.3\left(\mathrm{CH}_{2}\right), 29.5\left(\mathrm{CH}_{2}\right), 29.6\left(\mathrm{CH}_{2}\right), 30.8\left(\mathrm{CH}_{2}\right), 31.8\left(\mathrm{CH}_{2}\right), 32.0$ $\left(\mathrm{CH}_{2}\right), 34.1\left(\mathrm{CH}_{2}\right), 61.8\left(\mathrm{CH}_{2}\right), 132.8(\mathrm{C}), 134.4(\mathrm{C})$. HRMS-EI $(\mathrm{m} / \mathrm{z})$ : $[\mathrm{M}]^{+}$calcd for $\mathrm{C}_{17} \mathrm{H}_{34} \mathrm{O}$, 254.2610; found, 254.2605 . 
2-(1-Phenylbutan-2-ylidene)heptan-1-ol [6kc].<smiles>CCCCCC(CCCC)=C(CC)CCCC</smiles>

$4 \mathrm{kc}$<smiles>CCCCCC(CO)=C(CO)Cc1ccccc1</smiles>

$6 \mathrm{kc}$

The reaction was conducted with $79.1 \mathrm{mg}(0.500 \mathrm{mmol})$ of $\mathbf{1 k}$ according to the GP4. In order to remove an undesired boryl substitution product of the alkyl halide and protoboration product of the allene for the further purification and the determination of the regio- and stereoselectivities, the oxidation of the boryl groups was performed. The corresponding alcohol product $\mathbf{6 k \mathbf { c }}$ was obtained in $67 \%$ yield with $E / Z=50: 50,4: 5=>95: 5(82.5 \mathrm{mg}, 0.335 \mathrm{mmol}$, colorless oil).

${ }^{1} \mathrm{H}$ NMR (392 MHz, $\mathrm{CDCl}_{3}, \delta$ ): 0.80-1.04 (m, 6H), 1.17-1.63 (m, 7H), 1.96-2.10 (m, 2H), 2.14$2.31(\mathrm{~m}, 2 \mathrm{H}), 3.44$ and 3.50 (s, 2H, isomers), $4.18(\mathrm{~d}, J=5.9 \mathrm{~Hz}, 2 \mathrm{H}), 7.09-7.21(\mathrm{~m}, 3 \mathrm{H}), 7.21-7.30$ (m, 2H). ${ }^{13} \mathrm{C} \mathrm{NMR}\left(99 \mathrm{MHz}, \mathrm{CDCl}_{3}, \delta\right): 13.2$ and $14.0\left(\mathrm{CH}_{3}\right.$, isomers), 14.1 and $14.2\left(\mathrm{CH}_{3}\right.$, isomers), 22.5 and $22.6\left(\mathrm{CH}_{2}\right.$, isomers $), 24.3$ and $24.8\left(\mathrm{CH}_{2}\right.$, isomers $), 28.9$ and $29.0\left(\mathrm{CH}_{2}\right.$, isomers $), 30.3$ and $30.7\left(\mathrm{CH}_{2}\right.$, isomers), 32.1 and $32.2\left(\mathrm{CH}_{2}\right.$, isomers $), 36.4$ and $36.8\left(\mathrm{CH}_{2}\right.$, isomers $), 61.5$ and $62.0\left(\mathrm{CH}_{2}\right.$, isomers), $125.8(\mathrm{CH}), 128.2$ and $128.3(\mathrm{CH}$, isomers), 128.3 and $128.5(\mathrm{CH}$, isomers $), 134.6$ and 134.7 ( $C$, isomers), 137.8 and 138.1 ( $C$, isomers), 140.2 and $140.6\left(C\right.$, isomers). HRMS-EI $(m / z):[\mathrm{M}]^{+}$calcd for $\mathrm{C}_{17} \mathrm{H}_{26} \mathrm{O}$, 246.1984; found, 246.1983 .

(E)-2-(2-Ethyl-3,4,4-trimethylpent-2-en-1-yl)-4,4,5,5-tetramethyl-1,3,2-dioxaborolane $[(E)-4 \mathrm{bb}]$.<smiles>CC(C)=C(CB1OC(C)(C)C(C)(C)O1)C(C)(C)C</smiles>

The reaction was conducted with $55.1 \mathrm{mg}(0.500 \mathrm{mmol})$ of $\mathbf{1 b}$ according to the GP4. The product (E)-4bb was obtained in 75\% yield with $E / Z=>95: 5, \mathbf{4 : 5}=>95: 5(99.5 \mathrm{mg}, 0.374 \mathrm{mmol}$, colorless oil).

${ }^{1} \mathrm{H}$ NMR $\left(392 \mathrm{MHz}, \mathrm{CDCl}_{3}, \delta\right): 0.93(\mathrm{t}, J=7.4 \mathrm{~Hz}, 3 \mathrm{H}), 1.13(\mathrm{~s}, 9 \mathrm{H}), 1.23(\mathrm{~s}, 12 \mathrm{H}), 1.63(\mathrm{~s}, 3 \mathrm{H})$, 1.83 (s, 2H), 2.01 (q, $J=7.5 \mathrm{~Hz}, 2 \mathrm{H}) .{ }^{13} \mathrm{C}$ NMR $\left(99 \mathrm{MHz}, \mathrm{CDCl}_{3}, \delta\right): 12.3\left(\mathrm{CH}_{3}\right), 16.6\left(\mathrm{CH}_{3}\right), 19.4$ (br, B- $\left.\mathrm{CH}_{2}\right), 24.7\left(\mathrm{CH}_{3}\right), 30.4\left(\mathrm{CH}_{2}\right), 30.7\left(\mathrm{CH}_{3}\right), 35.6(\mathrm{C}), 82.9(\mathrm{C}), 130.8(\mathrm{C}), 133.3(\mathrm{C}) .{ }^{11} \mathrm{~B}$ NMR (126 MHz, $\left.\mathrm{CDCl}_{3}, \delta\right): 33.1$ (br, s). HRMS-EI $(m / z)$ : [M] ${ }^{+}$calcd for $\mathrm{C}_{16} \mathrm{H}_{31}{ }^{11} \mathrm{BO}_{2}, 266.2420$; found, 266.2419 . 
(E)-2-[2-(3,3-Dimethylbutan-2-ylidene)heptyl]-4,4,5,5-tetramethyl-1,3,2-dioxaborolane $[(E)-4 b c]$.

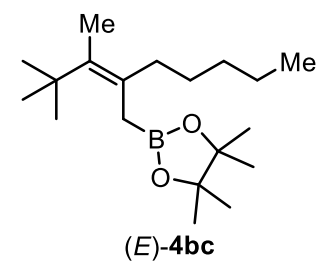

The reaction was conducted with $55.3 \mathrm{mg}(0.502 \mathrm{mmol})$ of $\mathbf{1 b}$ according to the GP4. The product (E)-4bc was obtained in 83\% yield with $E / Z=>95: 5, \mathbf{4 : 5}=>95: 5(128.3 \mathrm{mg}, 0.416 \mathrm{mmol}$, colorless oil).

${ }^{1} \mathrm{H} \mathrm{NMR}\left(396 \mathrm{MHz}, \mathrm{CDCl}_{3}, \delta\right): 0.89(\mathrm{t}, J=6.9 \mathrm{~Hz}, 3 \mathrm{H}), 1.13(\mathrm{~s}, 9 \mathrm{H}), 1.19-1.41(\mathrm{~m}, 6 \mathrm{H}), 1.23(\mathrm{~s}$, $12 \mathrm{H}), 1.62(\mathrm{~s}, 3 \mathrm{H}), 1.84(\mathrm{~s}, 2 \mathrm{H}), 1.93-2.01(\mathrm{~m}, 2 \mathrm{H}) .{ }^{13} \mathrm{C} \mathrm{NMR}\left(100 \mathrm{MHz}, \mathrm{CDCl}_{3}, \delta\right): 14.1\left(\mathrm{CH}_{3}\right), 16.9$ $\left(\mathrm{CH}_{3}\right), 20.0\left(\mathrm{br}, \mathrm{B}-\mathrm{CH}_{2}\right), 22.7\left(\mathrm{CH}_{2}\right), 24.7\left(\mathrm{CH}_{3}\right), 27.8\left(\mathrm{CH}_{2}\right), 30.7\left(\mathrm{CH}_{3}\right), 32.3\left(\mathrm{CH}_{2}\right), 35.6(\mathrm{C}), 37.7$ $\left(\mathrm{CH}_{2}\right), 82.9(\mathrm{C}), 129.7(\mathrm{C}), 133.6(\mathrm{C}) .{ }^{11} \mathrm{~B}$ NMR $\left(126 \mathrm{MHz}, \mathrm{CDCl}_{3}, \delta\right): 33.1$ (br, s). HRMS-EI $(\mathrm{m} / \mathrm{z})$ : $[\mathrm{M}]^{+}$calcd for $\mathrm{C}_{19} \mathrm{H}_{37}{ }^{11} \mathrm{BO}_{2}, 308.2890$; found, 308.2886.

(E)-2-[2-(3,3-Dimethylbutan-2-ylidene)dodecyl]-4,4,5,5-tetramethyl-1,3,2-dioxaborolane [(E)-4be].

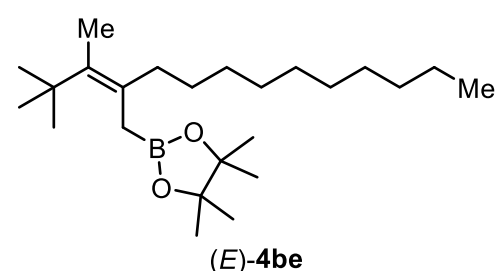

The reaction was conducted with $55.0 \mathrm{mg}(0.499 \mathrm{mmol})$ of $\mathbf{1 b}$ according to the GP4. The product (E)-4be was obtained in 88\% yield with $E / Z=>95: 5,4: 5=>95: 5(166.9 \mathrm{mg}, 0.441 \mathrm{mmol}$, colorless oil).

${ }^{1} \mathrm{H} \mathrm{NMR}\left(392 \mathrm{MHz}, \mathrm{CDCl}_{3}, \delta\right): 0.88$ (t, $\left.J=7.1 \mathrm{~Hz}, 3 \mathrm{H}\right), 1.13$ (s, 9H), 1.18-1.44 (m, 16H), 1.23 (s, $12 \mathrm{H}), 1.62$ (s, 3H), 1.83 (s, 2H), 1.96 (t, $J=7.8 \mathrm{~Hz}, 2 \mathrm{H}) .{ }^{13} \mathrm{C} \mathrm{NMR}\left(99 \mathrm{MHz}, \mathrm{CDCl}_{3}, \delta\right): 14.1\left(\mathrm{CH}_{3}\right)$, $16.9\left(\mathrm{CH}_{3}\right), 20.0\left(\mathrm{br}, \mathrm{B}-\mathrm{CH}_{2}\right), 22.7\left(\mathrm{CH}_{2}\right), 24.7\left(\mathrm{CH}_{3}\right), 28.1\left(\mathrm{CH}_{2}\right), 29.3\left(\mathrm{CH}_{2}\right), 29.6\left(\mathrm{CH}_{2}\right), 29.7\left(\mathrm{CH}_{2}\right)$, $30.0\left(\mathrm{CH}_{2}\right), 30.7\left(\mathrm{CH}_{3}\right), 31.9\left(\mathrm{CH}_{2}\right), 35.6(\mathrm{C}), 37.7(\mathrm{C}), 82.9(\mathrm{C}), 129.7(\mathrm{C}), 133.6(\mathrm{C}) .{ }^{11} \mathrm{~B}$ NMR (126 $\left.\mathrm{MHz}, \mathrm{CDCl}_{3}, \delta\right): 33.3$ (br, s). HRMS-EI $(\mathrm{m} / \mathrm{z})$ : [M] ${ }^{+}$calcd for $\mathrm{C}_{24} \mathrm{H}_{47}{ }^{11} \mathrm{BO}_{2}, 378.3674$; found, 378.3666 . 
(E)-2-(2-Isobutyl-3,4,4-trimethylpent-2-en-1-yl)-4,4,5,5-tetramethyl-1,3,2-dioxaborolane $[(E)-4 b f]$.

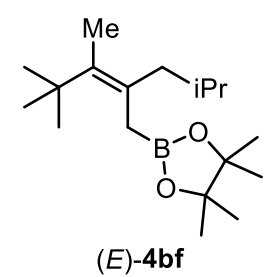

The reaction was conducted with $55.2 \mathrm{mg}(0.501 \mathrm{mmol})$ of $\mathbf{1 b}$ according to the GP4. The product (E)-4bf was obtained in 81\% yield with $E / Z=91: 9, \mathbf{4 : 5}=>95: 5$ (119.7 mg, $0.406 \mathrm{mmol}$, colorless oil).

${ }^{1} \mathrm{H}$ NMR $\left(392 \mathrm{MHz}, \mathrm{CDCl}_{3}, \delta\right): 0.86$ (d, $\left.J=6.3 \mathrm{~Hz}, 6 \mathrm{H}\right), 1.15$ (s, 9H), 1.23 (s, 12H), 1.64 (s, 3H), $1.69-1.83(\mathrm{~m}, 1 \mathrm{H}), 1.87$ (s, 2H), 1.95 (d, $J=7.4 \mathrm{~Hz}, 2 \mathrm{H}) .{ }^{13} \mathrm{C} \mathrm{NMR}\left(99 \mathrm{MHz}, \mathrm{CDCl}_{3}, \delta\right): 17.6\left(\mathrm{CH}_{3}\right)$, 20.3 (br, B- $\left.\mathrm{CH}_{2}\right), 22.5\left(\mathrm{CH}_{3}\right), 24.7\left(\mathrm{CH}_{3}\right), 27.6(\mathrm{CH}), 30.9\left(\mathrm{CH}_{3}\right), 35.8(\mathrm{C}), 45.8\left(\mathrm{CH}_{2}\right), 82.8(\mathrm{C}), 128.8$ (C), $134.8(C) .{ }^{11} \mathrm{~B}$ NMR $\left(126 \mathrm{MHz}, \mathrm{CDCl}_{3}, \delta\right): 33.1(\mathrm{br}, \mathrm{s})$. HRMS-EI $(\mathrm{m} / \mathrm{z})$ : [M] ${ }^{+}$calcd for $\mathrm{C}_{18} \mathrm{H}_{35}{ }^{11} \mathrm{BO}_{2}, 294.2733$; found, 294.2732 .

\section{(E)-2-(2-Benzyl-3,4,4-trimethylpent-2-en-1-yl)-4,4,5,5-tetramethyl-1,3,2-dioxaborolane} $[(E)-4 b g]$.

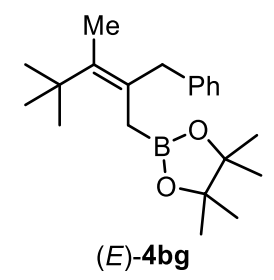

The reaction was conducted with $55.0 \mathrm{mg}(0.500 \mathrm{mmol})$ of $\mathbf{1 b}$ according to the GP4. The product (E)-4bg was obtained in 55\% yield with $E / Z=>95: 5, \mathbf{4 : 5}=>95: 5(90.3 \mathrm{mg}, 0.275 \mathrm{mmol}$, colorless oil).

${ }^{1} \mathrm{H}$ NMR (399 MHz, $\left.\mathrm{CDCl}_{3}, \delta\right): 1.21$ (s, 9H), $1.22(\mathrm{~s}, 12 \mathrm{H}), 1.69$ (s, 3H), $1.81(\mathrm{~s}, 2 \mathrm{H}), 3.46(\mathrm{~s}, 2 \mathrm{H})$, 7.10-7.18 (m, 3H), 7.23-7.28 (m, 2H). $\left.{ }^{13} \mathrm{C} \mathrm{NMR} \mathrm{(100} \mathrm{MHz,} \mathrm{CDCl}_{3}, \delta\right): 17.7\left(\mathrm{CH}_{3}\right), 20.2\left(\mathrm{br}, \mathrm{B}-\mathrm{CH}_{2}\right)$, $24.7\left(\mathrm{CH}_{3}\right), 30.7\left(\mathrm{CH}_{3}\right), 35.8(\mathrm{C}), 42.2\left(\mathrm{CH}_{2}\right), 82.9(\mathrm{C}), 125.5(\mathrm{CH}), 127.5(\mathrm{C}), 128.2(\mathrm{CH}), 128.2$ $(\mathrm{CH}), 136.6(\mathrm{C}), 140.7(\mathrm{C}) .{ }^{11} \mathrm{~B}$ NMR (126 MHz, $\left.\mathrm{CDCl}_{3}, \delta\right): 32.9$ (br, s). HRMS-EI $(\mathrm{m} / \mathrm{z}):[\mathrm{M}]^{+}$calcd for $\mathrm{C}_{21} \mathrm{H}_{33}{ }^{11} \mathrm{BO}_{2}, 328.2577$; found, 328.2578 . 
(E)-2-[2-(3-Methoxypropyl)-3,4,4-trimethylpent-2-en-1-yl]-4,4,5,5-tetramethyl-1,3,2dioxaborolane $[(E)-4 b h]$.

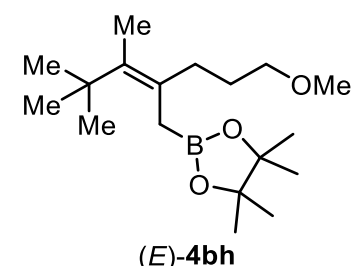

The reaction was conducted with $55.1 \mathrm{mg}(0.500 \mathrm{mmol})$ of $\mathbf{1 b}$ according to the GP4. The product (E)-4bh was obtained in 74\% yield with $E / Z=>95: 5, \mathbf{4 : 5}=>95: 5(114.8 \mathrm{mg}, 0.370 \mathrm{mmol}$, colorless oil).

${ }^{1} \mathrm{H}$ NMR (392 MHz, $\left.\mathrm{CDCl}_{3}, \delta\right): 1.13$ (s, 9H), 1.23 (s, 12H), 1.57-1.71 (m, 2H), 1.63 (s, 3H), 1.83 (s, 2H), $2.05(\mathrm{t}, J=7.8 \mathrm{~Hz}, 2 \mathrm{H}), 3.32(\mathrm{~s}, 3 \mathrm{H}), 3.35(\mathrm{t}, J=6.5 \mathrm{~Hz}, 2 \mathrm{H}) .{ }^{13} \mathrm{C} \mathrm{NMR}\left(99 \mathrm{MHz}, \mathrm{CDCl}_{3}, \delta\right)$ : $16.8\left(\mathrm{CH}_{3}\right), 19.9\left(\mathrm{br}, \mathrm{B}-\mathrm{CH}_{2}\right), 24.6\left(\mathrm{CH}_{3}\right), 27.9\left(\mathrm{CH}_{2}\right), 30.7\left(\mathrm{CH}_{3}\right), 33.7\left(\mathrm{CH}_{2}\right), 35.6(\mathrm{C}), 58.3\left(\mathrm{CH}_{3}\right)$, $72.7\left(\mathrm{CH}_{2}\right), 82.9(\mathrm{C}), 128.7(\mathrm{C}), 134.3(\mathrm{C}) .{ }^{11} \mathrm{~B}$ NMR (126 MHz, $\left.\mathrm{CDCl}_{3}, \delta\right): 33.1$ (br, s). HRMS-ESI $(\mathrm{m} / \mathrm{z}):[\mathrm{M}+\mathrm{Na}]^{+}$calcd for $\mathrm{C}_{18} \mathrm{H}_{35}{ }^{11} \mathrm{BO}_{3} \mathrm{Na}, 333.2575$; found, 333.2569 .

(Z)-6-[(tert-Butyldimethylsilyl)oxy]-2-(3,3-dimethylbutan-2-ylidene)hexan-1-ol [(Z)-6bi].

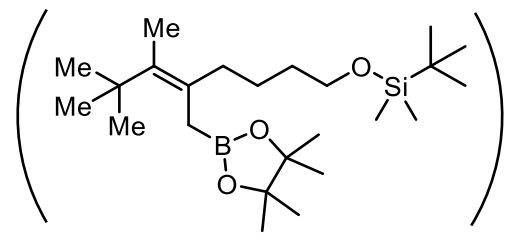

$(E)-4 \mathbf{b i}$<smiles>CC(CCCCO[Si](C)(C)C(C)(C)C)=C(CO)C(C)(C)C</smiles>

(Z)-6bi

The reaction was conducted with $55.3 \mathrm{mg}(0.502 \mathrm{mmol})$ of $\mathbf{1 b}$ according to the GP4. In order to remove an undesired boryl substitution product of the alkyl halide and protoboration product of the allene for the further purification and the determination of the regio- and stereoselectivities, the oxidation of the boryl groups was performed. The corresponding alcohol product (Z)-6bi was obtained in $44 \%$ yield with $E / Z=<5: 95,4: 5=>95: 5(69.6 \mathrm{mg}, 0.221 \mathrm{mmol}$, colorless oil $)$.

${ }^{1} \mathrm{H}$ NMR (396 MHz, $\left.\mathrm{CDCl}_{3}, \delta\right): 0.05$ (s, 6H), 0.89 (s, 9H), 1.18 (s, 9H), 1.35-1.47 (m, 2H), 1.48$1.62(\mathrm{~m}, 3 \mathrm{H}), 1.68(\mathrm{~s}, 3 \mathrm{H}), 2.13(\mathrm{t}, J=8.1 \mathrm{~Hz}, 2 \mathrm{H}), 3.62(\mathrm{t}, J=6.3 \mathrm{~Hz}, 2 \mathrm{H}), 4.23(\mathrm{~d}, J=5.9 \mathrm{~Hz}, 2 \mathrm{H})$. ${ }^{13} \mathrm{C} \mathrm{NMR}\left(99 \mathrm{MHz}, \mathrm{CDCl}_{3}, \delta\right):-5.3\left(\mathrm{CH}_{3}\right), 17.1\left(\mathrm{CH}_{3}\right), 18.3(\mathrm{C}), 25.0\left(\mathrm{CH}_{2}\right), 25.9\left(\mathrm{CH}_{3}\right), 31.7\left(\mathrm{CH}_{3}\right)$, $32.88\left(\mathrm{CH}_{2}\right), 32.95\left(\mathrm{CH}_{2}\right), 36.0(\mathrm{C}), 62.5\left(\mathrm{CH}_{2}\right), 63.0\left(\mathrm{CH}_{2}\right), 133.1(\mathrm{C}), 142.1(\mathrm{C})$. HRMS-EI $(\mathrm{m} / z)$ : $[\mathrm{M}-t \mathrm{Bu}]^{+}$calcd for $\mathrm{C}_{14} \mathrm{H}_{29} \mathrm{O}_{2} \mathrm{Si}$, 257.1937; found, 257.1938 . 
(Z)-2-(3-Chloropropyl)-3,4,4-trimethylpent-2-en-1-ol [(Z)-6bj].

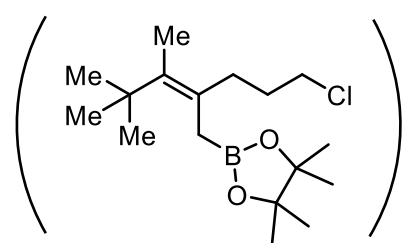

$(E)-\mathbf{4 b j}$<smiles>CC(C)(C)C(CCO)CCCCl</smiles>

(Z)-6bj

The reaction was conducted with $55.1 \mathrm{mg}(0.500 \mathrm{mmol})$ of $\mathbf{1 b}$ according to the GP4. In order to remove an undesired boryl substitution product of the alkyl halide and protoboration product of the allene for the further purification and the determination of the regio- and stereoselectivities, the oxidation of the boryl groups was performed. The corresponding alcohol product (Z)-6bj was obtained in $51 \%$ yield with $E / Z=<5: 95,4: 5=>95: 5(52.3 \mathrm{mg}, 0.255 \mathrm{mmol}$, colorless oil). The selectivity of $\mathrm{C}-\mathrm{C}$ bond formation at chloride and iodate was $86: 14$ (determined by ${ }^{1} \mathrm{H}$ NMR analysis).

${ }^{1} \mathrm{H}$ NMR (396 MHz, $\mathrm{CDCl}_{3}, \delta$ ): 1.19 (s, 9H), 1.30 (br-s, 1H), 1.71 (s, 3H), 1.79-1.98 (m, 2H), 2.19-2.35 (m, 2H), 3.54 (t, $J=6.5 \mathrm{~Hz}, 2 \mathrm{H}), 4.25$ (s, $2 \mathrm{H}) .{ }^{13} \mathrm{C} \mathrm{NMR}\left(100 \mathrm{MHz}, \mathrm{CDCl}_{3}, \delta\right): 17.2\left(\mathrm{CH}_{3}\right)$, $30.5\left(\mathrm{CH}_{2}\right), 31.7\left(\mathrm{CH}_{3}\right), 31.9\left(\mathrm{CH}_{2}\right), 36.1(\mathrm{C}), 45.2\left(\mathrm{CH}_{2}\right), 62.6\left(\mathrm{CH}_{2}\right), 131.6(C), 143.2(C)$. HRMS$\mathrm{EI}(\mathrm{m} / \mathrm{z}):[\mathrm{M}]^{+}$calcd for $\mathrm{C}_{11} \mathrm{H}_{21} \mathrm{ClO}, 204.1281$; found, 204.1281.

\section{6,6,6-Trifluoro-2-(4-phenylcyclohexylidene)hexan-1-ol (6la).}

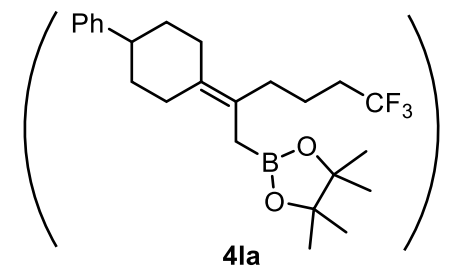

4la<smiles>OCC(CCCC(F)(F)F)=C1CCC(c2ccccc2)CC1</smiles>

6la

The reaction was conducted with $92.1 \mathrm{mg}(0.500 \mathrm{mmol})$ of 11 according to the GP4. In order to remove an undesired boryl substitution product of the alkyl halide and protoboration product of the allene for the further purification and the determination of the regio- and stereoselectivities, the oxidation of the boryl groups was performed. The corresponding alcohol product 6la was obtained in $69 \%$ yield with 4:5 = >95:5 (107.3 mg, $0.343 \mathrm{mmol}$, colorless oil $)$.

${ }^{1} \mathrm{H}$ NMR (392 MHz, $\left.\mathrm{CDCl}_{3}, \delta\right): 1.05-1.20$ (m, 1H), 1.41-1.60 (m, 2H), 1.62-1.74 (m, 2H), 1.91$2.18(\mathrm{~m}, 6 \mathrm{H}), 2.28(\mathrm{t}, J=8.1 \mathrm{~Hz}, 2 \mathrm{H}), 2.68-2.80(\mathrm{~m}, 2 \mathrm{H}), 2.84-2.93(\mathrm{~m}, 1 \mathrm{H}), 4.14-4.24(\mathrm{~m}, 2 \mathrm{H})$, 7.16-7.23 (m, 3H), 7.25-7.33 (m, 2H). ${ }^{13} \mathrm{C}$ NMR (99 MHz, $\left.\mathrm{CDCl}_{3}, \delta\right): 21.7\left(\mathrm{CH}_{2}\right), 29.5\left(\mathrm{CH}_{2}\right), 30.1$ $\left(\mathrm{CH}_{2}\right), 30.3\left(\mathrm{CH}_{2}\right), 33.5\left(\mathrm{q}, J=28.6 \mathrm{~Hz}, \mathrm{CH}_{2}\right), 35.4\left(\mathrm{CH}_{2}\right), 35.6\left(\mathrm{CH}_{2}\right), 44.5(\mathrm{CH}), 61.6\left(\mathrm{CH}_{2}\right), 126.0$ $(\mathrm{CH}), 126.7(\mathrm{CH}), 127.2$ (q, $J=277.9 \mathrm{~Hz}, C), 128.3(\mathrm{CH}), 128.6(C), 138.7(C), 146.4(C)$. HRMS-EI $(\mathrm{m} / \mathrm{z}):[\mathrm{M}]^{+}$calcd for $\mathrm{C}_{18} \mathrm{H}_{23} \mathrm{~F}_{3} \mathrm{O}, 312.1701$; found, 312.1703 . 
4,4,5,5-Tetramethyl-2-[(E)-6,6,6-trifluoro-2-\{(8S,9S,13S,14S)-3-methoxy-13-methyl$6,7,8,9,11,12,13,14,15,16-d e c a h y d r o-17 H$-cyclopenta[a]phenanthren-17-ylidene\}hexyl]-1,3,2dioxaborolane $[(E)-4 \mathrm{ma}]$.

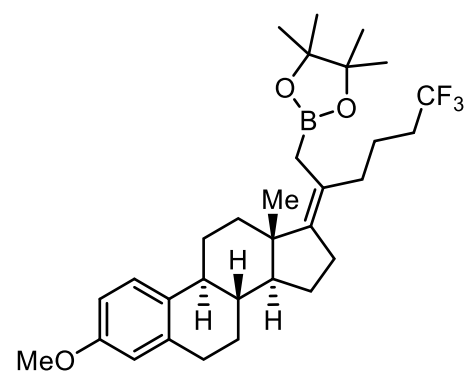

(E)-4ma

The reaction was conducted with $147.2 \mathrm{mg}(0.500 \mathrm{mmol})$ of $1 \mathrm{~m}$ according to the GP4. The product (E)-4ma was obtained in $90 \%$ yield with $E / Z=>95: 5,4: 5=>95: 5(240.6 \mathrm{mg}, 0.452 \mathrm{mmol}$, colorless oil).

${ }^{1} \mathrm{H}$ NMR (396 MHz, $\left.\mathrm{CDCl}_{3}, \delta\right): 0.87$ (s, 3H), 1.02-1.82 (m, 10H), $1.24(\mathrm{~s}, 12 \mathrm{H}), 1.83-2.39$ (m, $11 \mathrm{H}), 2.78-2.93(\mathrm{~m}, 2 \mathrm{H}), 3.76(\mathrm{~s}, 3 \mathrm{H}), 6.62(\mathrm{~d}, J=2.8 \mathrm{~Hz}, 1 \mathrm{H}), 6.70(\mathrm{dd}, J=8.7 \mathrm{~Hz}, 2.8 \mathrm{~Hz}, 1 \mathrm{H})$, $7.20(\mathrm{~d}, J=8.3 \mathrm{~Hz}, 1 \mathrm{H}) .{ }^{13} \mathrm{C} \mathrm{NMR}\left(100 \mathrm{MHz}, \mathrm{CDCl}_{3}, \delta\right): 16.1$ (br, B-CH$), 16.4\left(\mathrm{CH}_{3}\right), 19.6\left(\mathrm{CH}_{2}\right)$, $24.0\left(\mathrm{CH}_{2}\right), 24.6\left(\mathrm{CH}_{3}\right), 24.7\left(\mathrm{CH}_{3}\right), 27.0\left(\mathrm{CH}_{2}\right), 27.5\left(\mathrm{CH}_{2}\right), 29.5\left(\mathrm{CH}_{2}\right), 29.8\left(\mathrm{CH}_{2}\right), 33.4(\mathrm{q}, J=28.1$ $\left.\mathrm{Hz}, \mathrm{CH}_{2}\right), 35.3\left(\mathrm{CH}_{2}\right), 37.7\left(\mathrm{CH}_{2}\right), 38.2(\mathrm{CH}), 43.5(\mathrm{CH}), 44.5(\mathrm{C}), 55.0(\mathrm{CH}), 55.1(\mathrm{CH}), 83.0(\mathrm{C})$, $111.3(\mathrm{CH}), 113.6(\mathrm{CH}), 123.6(C), 126.1(\mathrm{CH}), 127.4(\mathrm{q}, J=277.5 \mathrm{~Hz}, C), 132.8(C), 137.9(C), 143.3$ (C), 157.3 (C). ${ }^{11} \mathrm{~B}$ NMR (126 MHz, $\mathrm{CDCl}_{3}, \delta$ ): 33.0 (br, s). HRMS-EI $(\mathrm{m} / \mathrm{z})$ : [M] $]^{+}$calcd for $\mathrm{C}_{31} \mathrm{H}_{44}{ }^{11} \mathrm{BF}_{3} \mathrm{O}_{3}, 532.3341$; found, 532.3322 . 
2-\{(E)-2-[(8S,9S,13S,14S)-3-Methoxy-13-methyl-6,7,8,9,11,12,13,14,15,16-decahydro-17Hcyclopenta[a]phenanthren-17-ylidene]butyl\}-4,4,5,5-tetramethyl-1,3,2-dioxaborolane $[(E)-4 m b]$.

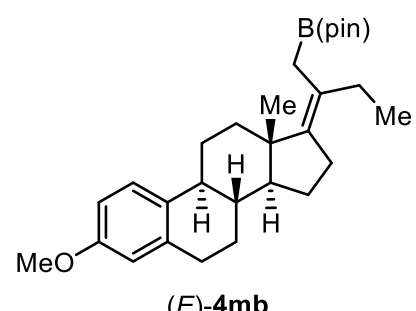

The reaction was conducted with $147.2 \mathrm{mg}(0.500 \mathrm{mmol})$ of $\mathbf{1 m}$ according to the GP4. The product (E)-4mb was obtained in 78\% yield with $E / Z=>95: 5,4: 5=>95: 5(176.2 \mathrm{mg}, 0.391 \mathrm{mmol}$, colorless oil).

${ }^{1} \mathrm{H}$ NMR (392 MHz, $\left.\mathrm{CDCl}_{3}, \delta\right): 0.86(\mathrm{~s}, 3 \mathrm{H}), 0.94(\mathrm{t}, J=7.4 \mathrm{~Hz}, 3 \mathrm{H}), 1.16-1.59(\mathrm{~m}, 5 \mathrm{H}), 1.25(\mathrm{~s}$, 12H), 1.62-1.80 (m, 3H), 1.84-2.05 (m, 4H), 2.14-2.39 (m, 5H), 2.77-2.97 (m, 2H), 3.77 (s, 3H), $6.63(\mathrm{~d}, J=2.7 \mathrm{~Hz}, 1 \mathrm{H}), 6.70(\mathrm{dd}, J=8.2,2.7 \mathrm{~Hz}, 1 \mathrm{H}), 7.21(\mathrm{~d}, J=9.0 \mathrm{~Hz}, 1 \mathrm{H}) .{ }^{13} \mathrm{C}$ NMR $(99 \mathrm{MHz}$, $\left.\mathrm{CDCl}_{3}, \delta\right): 11.8\left(\mathrm{CH}_{3}\right), 15.8\left(\mathrm{br}, \mathrm{B}-\mathrm{CH}_{2}\right), 16.5\left(\mathrm{CH}_{3}\right), 24.1\left(\mathrm{CH}_{2}\right), 24.6\left(\mathrm{CH}_{3}\right), 24.8\left(\mathrm{CH}_{3}\right), 27.1\left(\mathrm{CH}_{2}\right)$, $27.6\left(\mathrm{CH}_{2}\right), 29.06\left(\mathrm{CH}_{2}\right), 29.09\left(\mathrm{CH}_{2}\right), 29.9\left(\mathrm{CH}_{2}\right), 37.8\left(\mathrm{CH}_{2}\right), 38.2(\mathrm{CH}), 43.6(\mathrm{CH}), 44.2(C), 55.1$ $(\mathrm{CH}), 55.2\left(\mathrm{CH}_{3}\right), 82.9(\mathrm{C}), 111.4(\mathrm{CH}), 113.6(\mathrm{CH}), 126.2(\mathrm{CH}), 126.7(\mathrm{C}), 133.0(\mathrm{C}), 138.0(\mathrm{C})$, 141.1 (C), 157.3 (C). ${ }^{11} \mathrm{~B}$ NMR (126 MHz, $\left.\mathrm{CDCl}_{3}, \delta\right): 33.0$ (br, s). HRMS-EI $(\mathrm{m} / \mathrm{z})$ : [M] ${ }^{+}$calcd for $\mathrm{C}_{29} \mathrm{H}_{43}{ }^{11} \mathrm{BO}_{3}, 450.3310$; found, 450.3306 . 


\section{Large Scale Reaction and Synthetic Applications of Borylation Products}

\subsection{Gram-scale reaction.}

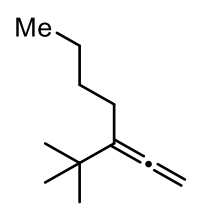

1h $(6.0 \mathrm{mmol})$

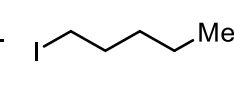

2c (2.0 equiv)
$5 \mathrm{~mol} \% \mathrm{CuCl} / \mathrm{Xantphos}$

(pin)B-B(pin) (3) (1.2 equiv)

$\mathrm{K}(\mathrm{O}-t-\mathrm{Bu})$ (1.2 equiv) DMF $(0.5 \mathrm{M}), 30^{\circ} \mathrm{C}, 48 \mathrm{~h}$

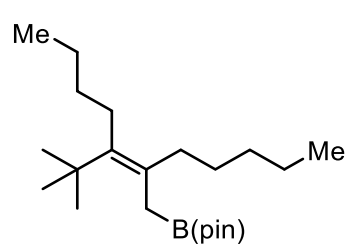

(E)-4hc

$88 \%$ yield $(1.86 \mathrm{~g})$

$\mathrm{CuCl}$ (29.7 mg, $0.30 \mathrm{mmol})$, bis(pinacolato)diboron (3) (1.83 g, $7.20 \mathrm{mmol})$, Xantphos (174 mg, $0.300 \mathrm{mmol})$ and $\mathrm{K}(\mathrm{O}-\mathrm{t}-\mathrm{Bu})(808 \mathrm{mg}, 7.20 \mathrm{mmol})$ were placed in a round-bottomed flask under a nitrogen atmosphere. Dry DMF $(12.0 \mathrm{~mL})$ was added to the flask through the rubber septum using a syringe. After stirring for $30 \mathrm{~min}$ at room temperature, $\mathbf{1 h}(914 \mathrm{mg}, 6.00 \mathrm{mmol})$ and $\mathbf{2 c}(2.38 \mathrm{~g}, 12.0$ mmol) were added to the mixture. Then, the reaction mixture was heated to $30{ }^{\circ} \mathrm{C}$. After the reaction was completed, the reaction mixture was quenched with water and extracted with EtOAc three times. The combined organic layer was dried over $\mathrm{MgSO}_{4}$. After filtration, the solvents were removed under reduced pressure. The crude material was purified by flash column chromatography $\left(\mathrm{SiO}_{2}\right.$, $\mathrm{Et}_{2} \mathrm{O} /$ hexane, 0:100-3:97) to give the corresponding borylation product $(E)-4$ he in $88 \%$ yield with $E / Z$ $=>95: 5,4: 5=>95: 5(1.86 \mathrm{~g}, 5.29 \mathrm{mmol}$, colorless oil $)$. 


\subsection{Synthesis of (Z)-3-(tert-butyl)-2-pentylhept-2-en-1-ol [(Z)-6hc].}

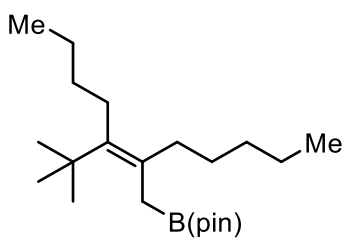

(E)-4hc

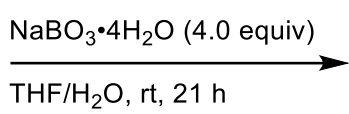

$\mathrm{THF} / \mathrm{H}_{2} \mathrm{O}, \mathrm{rt}, 21 \mathrm{~h}$

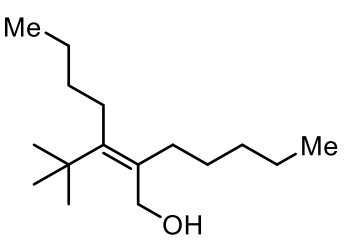

(Z)-6hc

$89 \%$ yield

In a reaction vial, $(E)-4 h \mathbf{h}(70.1 \mathrm{mg}, 0.200 \mathrm{mmol})$ was dissolved in THF $(2.0 \mathrm{~mL})$, then $\mathrm{H}_{2} \mathrm{O}(2.0$ $\mathrm{mL})$ and $\mathrm{NaBO}_{3} \bullet 4 \mathrm{H}_{2} \mathrm{O}(300 \mathrm{mg}, 0.80 \mathrm{mmol})$ were subsequently added to the reaction mixture at $\mathrm{rt}$ under air. The reaction mixture was stirred at $\mathrm{rt}$ for $21 \mathrm{~h}$. The reaction mixture was then quenched by $\mathrm{H}_{2} \mathrm{O}$ and extracted three times with EtOAc. The combined organic layer was dried over $\mathrm{MgSO}_{4}$ followed by filtration. The crude material was purified by silica gel chromatography (EtOAc/hexane, 0:100-8:92) to give the corresponding alcohol (Z)-6hc in 89\% yield with $E / Z=<5: 95$ (42.9 mg, 0.178 mmol, colorless oil).

${ }^{1} \mathrm{H}$ NMR $\left(392 \mathrm{MHz}, \mathrm{CDCl}_{3}, \delta\right): 0.86-0.97$ (m, 6H), 1.20 (s, 9H), 1.24-1.45 (m, 11H), 2.01-2.12 (m, 4H), 4.26 (s, 2H). ${ }^{13} \mathrm{C}$ NMR (99 MHz, $\left.\mathrm{CDCl}_{3}, \delta\right): 13.9\left(\mathrm{CH}_{3}\right), 14.1\left(\mathrm{CH}_{3}\right), 22.6\left(\mathrm{CH}_{2}\right), 23.4\left(\mathrm{CH}_{2}\right)$, 29.2 $\left(\mathrm{CH}_{2}\right), 30.8\left(\mathrm{CH}_{2}\right), 32.1\left(\mathrm{CH}_{2}\right), 32.4\left(\mathrm{CH}_{3}\right.$ and $\left.\mathrm{CH}_{2}\right), 33.4\left(\mathrm{CH}_{2}\right), 35.8(\mathrm{C}), 62.3\left(\mathrm{CH}_{2}\right), 134.2(\mathrm{C})$, $146.2(\mathrm{C})$. HRMS-EI $(\mathrm{m} / \mathrm{z})$ : $[\mathrm{M}]^{+}$calcd for $\mathrm{C}_{16} \mathrm{H}_{32} \mathrm{O}, 240.2453$; found, 240.2454. 


\subsection{Synthesis of tert-butyl (Z)-(3-(tert-butyl)-2-pentylhept-2-en-1-yl)carbamate $[(Z)-7$ hc $]{ }^{10}$}

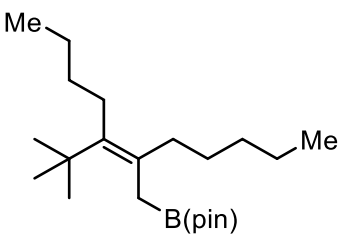

(E)-4hc

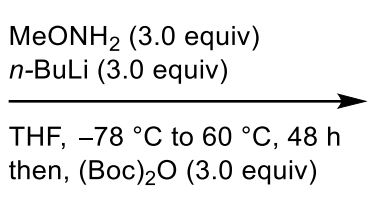

then, $(\mathrm{Boc})_{2} \mathrm{O}$ (3.0 equiv)

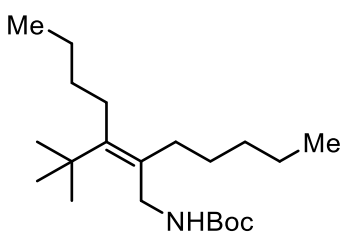

(Z)-7hc

$46 \%$ yield

According to the literature procedure, the amination of $(E)-\mathbf{4 h c}$ and preparation of $\mathrm{MeONH}_{2}$ solution in THF $(0.80 \mathrm{M})$ were conducted. ${ }^{10}$ In an oven-dried reaction vial, $\mathrm{MeONH}_{2}(0.80 \mathrm{M}$ in THF, $751 \mu \mathrm{L}, 0.60 \mathrm{mmol})$ was diluted with THF $(600 \mu \mathrm{L})$. After the mixture was cooled to $-78{ }^{\circ} \mathrm{C}, n$-BuLi $(1.57 \mathrm{M}$ in hexane, $375 \mu \mathrm{L}, 0.60 \mathrm{mmol}$ ) was added dropwise under a nitrogen atmosphere. Then $(E)$ 4he $(70.1 \mathrm{mg}, 0.20 \mathrm{mmol})$ in THF $(400 \mu \mathrm{L})$ was added dropwise to the solution and stirred at $60^{\circ} \mathrm{C}$. After $48 \mathrm{~h},(\mathrm{Boc})_{2} \mathrm{O}(138 \mu \mathrm{L}, 0.60 \mathrm{mmol})$ was added to the mixture and stirred for $2 \mathrm{~h}$ at rt. The mixture was then quenched by addition of $\mathrm{H}_{2} \mathrm{O}$ and extracted three times with EtOAc. The combined organic layer was dried over $\mathrm{MgSO}_{4}$ followed by filtration and evaporation. After purification by flash silica gel column chromatography ( $\mathrm{Et}_{2} \mathrm{O} /$ hexane, :100-4:96), (Z)-7he was further purified by oxidation reaction with $\mathrm{NaBO}_{3} \cdot 4 \mathrm{H}_{2} \mathrm{O}(300 \mathrm{mg}, 0.80 \mathrm{mmol})$ in THF $(2.0 \mathrm{~mL})$ and $\mathrm{H}_{2} \mathrm{O}(2.0 \mathrm{~mL})$ to remove unreacted starting material. After the oxidation reaction, the crude material was purified by silica gel chromatography ( $\mathrm{Et}_{2} \mathrm{O} /$ hexane, 0:100-4:96) to give the pure (Z)-7hc in $46 \%$ yield with $E / Z=<5: 95$ (30.8 mg, $0.091 \mathrm{mmol}$, colorless oil).

${ }^{1} \mathrm{H}$ NMR (392 MHz, $\left.\mathrm{CDCl}_{3}, \delta\right): 0.84-0.95(\mathrm{~m}, 6 \mathrm{H}), 1.17(\mathrm{~s}, 9 \mathrm{H}), 1.22-1.41(\mathrm{~m}, 10 \mathrm{H}), 1.45(\mathrm{~s}, 9 \mathrm{H})$, 1.89-1.97 (m, 2H), 1.98-2.08 (m, 2H), 3.88 (d, $J=5.4 \mathrm{~Hz}, 2 \mathrm{H}), 4.22-4.34$ (m, 1H). ${ }^{13} \mathrm{C}$ NMR (99 $\left.\mathrm{MHz}, \mathrm{CDCl}_{3}, \delta\right): 13.9\left(\mathrm{CH}_{3}\right), 14.1\left(\mathrm{CH}_{3}\right), 22.5\left(\mathrm{CH}_{2}\right), 23.4\left(\mathrm{CH}_{2}\right), 28.4\left(\mathrm{CH}_{3}\right), 29.1\left(\mathrm{CH}_{2}\right), 30.8\left(\mathrm{CH}_{2}\right)$, $32.1\left(\mathrm{CH}_{3}\right), 32.3\left(\mathrm{CH}_{2}\right), 32.4\left(\mathrm{CH}_{2}\right), 33.5\left(\mathrm{CH}_{2}\right), 35.6(C), 42.1\left(\mathrm{CH}_{2}\right), 79.1(C), 131.3(C), 146.1(C)$, $156.0(C)$. HRMS-ESI $(m / z)$ : [M+Na] $]^{+}$calcd for $\mathrm{C}_{21} \mathrm{H}_{41} \mathrm{O}_{2} \mathrm{NNa}$, 362.3030; found, 362.3029 . 
6.4. Synthesis of (E)-2-(4-(tert-butyl)-3-pentyloct-3-en-1-yl)-4,4,5,5-tetramethyl-1,3,2dioxaborolane $[(E)-8 h c] .{ }^{11}$

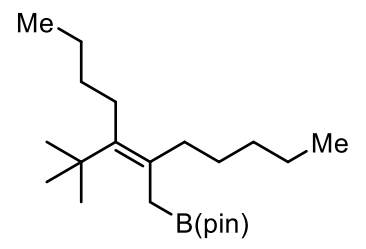

$(E)-4 h c$
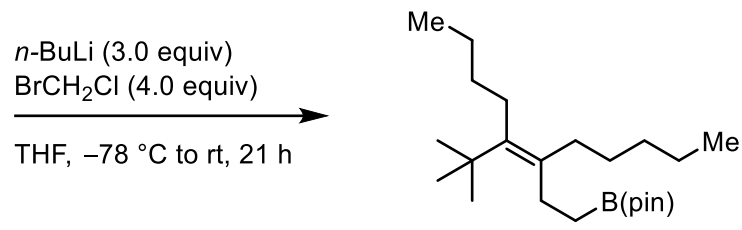

(E)-8hc $73 \%$ yield

Homologation reaction of $(E)$-4he was performed according to the literature procedure. In an ovendried reaction vial, $(E)-4 \mathrm{he}(70.1 \mathrm{mg}, 0.200 \mathrm{mmol})$ and $\mathrm{BrCH}_{2} \mathrm{Cl}(53.6 \mu \mathrm{L}, 0.800 \mathrm{mmol})$ were dissolved in THF $(1.2 \mathrm{~mL})$ in a nitrogen atmosphere, and the mixture was cooled to $-78{ }^{\circ} \mathrm{C} . n$-BuLi (1.6 $\mathrm{M}$ in hexane, $375 \mu \mathrm{L}, 0.600 \mathrm{mmol}$ ) was then added dropwise to the reaction mixture, and the mixture was stirred at $\mathrm{rt}$ for $21 \mathrm{~h}$. The mixture was then quenched by the addition of a saturated aqueous $\mathrm{NH}_{4} \mathrm{Cl}$ solution and extracted three times with $\mathrm{Et}_{2} \mathrm{O}$. Next, the combined organic layer was washed with brine, dried over $\mathrm{MgSO}_{4}$ followed by filtration. After evaporation, the crude material was purified by silica gel chromatography $\left(\mathrm{Et}_{2} \mathrm{O} /\right.$ hexane, $\left.0: 100-3: 97\right)$ to give the corresponding boronate $(E)-8 \mathrm{hc}$ in $73 \%$ yield with $E / Z=>95: 5$ (52.8 $\mathrm{mg}, 0.145 \mathrm{mmol}$, colorless oil).

${ }^{1} \mathrm{H}$ NMR (392 MHz, $\left.\mathrm{CDCl}_{3}, \delta\right): 0.81-0.94(\mathrm{~m}, 8 \mathrm{H}), 1.16(\mathrm{~s}, 9 \mathrm{H}), 1.20-1.42(\mathrm{~m}, 10 \mathrm{H}), 1.25$ (s, 12H), 1.87-1.94 (m, 2H), 1.94-2.02 (m, 2H), 2.19-2.27 (m, 2H). $\left.{ }^{13} \mathrm{C} \mathrm{NMR} \mathrm{(99} \mathrm{MHz,} \mathrm{CDCl}_{3}, \delta\right): 11.1$ (br, B- $\left.\mathrm{CH}_{2}\right), 14.0\left(\mathrm{CH}_{3}\right), 14.1\left(\mathrm{CH}_{3}\right), 22.7\left(\mathrm{CH}_{2}\right), 23.4\left(\mathrm{CH}_{2}\right), 24.8\left(\mathrm{CH}_{3}\right), 26.9\left(\mathrm{CH}_{2}\right), 29.2\left(\mathrm{CH}_{2}\right), 30.7$ $\left(\mathrm{CH}_{2}\right), 31.9\left(\mathrm{CH}_{3}\right), 32.5\left(\mathrm{CH}_{2}\right), 32.7\left(\mathrm{CH}_{2}\right), 33.8\left(\mathrm{CH}_{2}\right), 35.5(\mathrm{C}), 82.8(\mathrm{C}), 137.1(\mathrm{C}), 139.4(\mathrm{C}) .{ }^{11} \mathrm{~B}$ NMR (126 MHz, $\mathrm{CDCl}_{3}, \delta$ ): 33.7 (br, s). HRMS-EI (m/z): [M] ${ }^{+}$calcd for $\mathrm{C}_{23} \mathrm{H}_{45}{ }^{11} \mathrm{BO}_{2}, 364.3517$; found, 364.3516 . 


\section{Procedure of Allylboration Reactions}

\subsection{Synthesis of 2-(tert-butyl)-2-butyl-3-methyleneoctan-1-ol (9a). ${ }^{12}$}

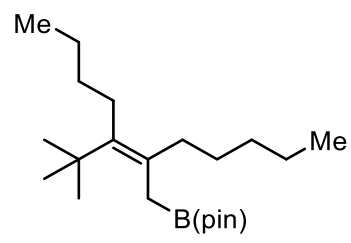

(E)-4hc

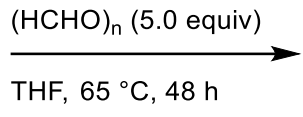

THF, $65^{\circ} \mathrm{C}, 48 \mathrm{~h}$

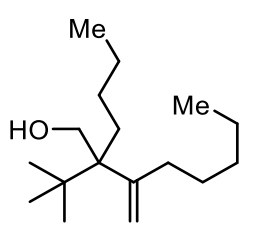

9a

Allylboration reaction of $(E)-4 h \mathbf{h e}$ was conducted according to the slightly modified literature procedure. In an oven-dried reaction vial, $(E)-4 \mathbf{h c}(70.1 \mathrm{mg}, 0.200 \mathrm{mmol})$ and paraformaldehyde $(30.0$ $\mathrm{mg}, 1.00 \mathrm{mmol})$ were dissolved in THF $(1.0 \mathrm{~mL})$ under a nitrogen atmosphere. The reaction mixture was stirred at $65{ }^{\circ} \mathrm{C}$ for $48 \mathrm{~h}$. The mixture was then quenched by the addition of triethanolamine $(10 \%$ solution in DCM) and extracted three times with DCM. The combined organic layer was washed with brine, dried over $\mathrm{MgSO}_{4}$ followed by filtration. After evaporation, the crude material was purified by silica gel chromatography $\left(\mathrm{Et}_{2} \mathrm{O} /\right.$ hexane, $\left.0: 100-5: 95\right)$ to give the corresponding homo-allyl alcohol 9a in $90 \%$ yield ( $45.8 \mathrm{mg}, 0.180 \mathrm{mmol}$, colorless oil).

${ }^{1} \mathrm{H}$ NMR (392 MHz, $\left.\mathrm{CDCl}_{3}, \delta\right): 0.92$ (s, 9H), 0.80-0.98 (m, 6H), 1.15-1.40 (m, 9H), 1.41-1.56 (m, 2H), $1.60-1.72(\mathrm{~m}, 2 \mathrm{H}), 1.98(\mathrm{t}, J=7.8 \mathrm{~Hz}, 2 \mathrm{H}), 3.76-3.93(\mathrm{~m}, 2 \mathrm{H}), 4.83(\mathrm{~s}, 1 \mathrm{H}), 5.16(\mathrm{~s}, 1 \mathrm{H}) .{ }^{13} \mathrm{C}$ NMR (99 MHz, $\left.\mathrm{CDCl}_{3}, \delta\right): 14.1\left(\mathrm{CH}_{3}\right), 14.2\left(\mathrm{CH}_{3}\right), 22.7\left(\mathrm{CH}_{2}\right), 24.1\left(\mathrm{CH}_{2}\right), 27.5\left(\mathrm{CH}_{3}\right), 27.9\left(\mathrm{CH}_{2}\right)$, $29.2\left(\mathrm{CH}_{2}\right), 30.3\left(\mathrm{CH}_{2}\right), 32.2\left(\mathrm{CH}_{2}\right), 33.5\left(\mathrm{CH}_{2}\right), 36.4(\mathrm{C}), 51.7(\mathrm{C}), 64.8\left(\mathrm{CH}_{2}\right), 112.5\left(\mathrm{CH}_{2}\right), 150.9$ (C). HRMS-FD $(\mathrm{m} / \mathrm{z})$ : [M] $]^{+}$calcd for $\mathrm{C}_{17} \mathrm{H}_{34} \mathrm{O}, 254.2610$; found, 254.2605 . 
7.2. Synthesis of 2-cyclohexyl-7,7,7-trifluoro-2-methyl-3-methylene-1-phenylheptan-1-ol (9b). ${ }^{13}$<smiles>CC(=C(CBr)CCCC(F)(F)F)C1CCCCC1</smiles>

(E)-4aa<smiles>O=Cc1ccccc1</smiles>

(1.5 equiv)<smiles>C=C(CCCC(F)(F)F)[C@](C)(Cl)[C@@H](O)c1ccccc1</smiles>

9b $75 \%$ yield

Allylboration reaction of $(E)$-4aa was conducted according to the slightly modified literature procedure. In an oven-dried reaction vial, $(E)-4 a a(38.3 \mathrm{mg}, 0.102 \mathrm{mmol})$ was dissolved in dry DCM $(200 \mu \mathrm{L})$ under a nitrogen atmosphere, and the solution was cooled to $-78{ }^{\circ} \mathrm{C}$. Then, corresponding aldehyde $(15.5 \mu \mathrm{L}, 0.15 \mathrm{mmol})$ was added to the reaction mixture, followed by the addition of $\mathrm{BF}_{3} \cdot \mathrm{OEt}_{2}(12.8 \mu \mathrm{L}, 0.10 \mathrm{mmol})$. After stirring for $3 \mathrm{~h}$ at $-78^{\circ} \mathrm{C}$, the reaction mixture was quenched by the addition of triethanolamine (10\% solution in DCM) and extracted three times with DCM. Next, the combined organic layer was washed with brine, dried over $\mathrm{MgSO}_{4}$ followed by filtration. After evaporation, the crude material was purified by silica gel chromatography (EtOAc/hexane, 0:100 3:97) to give the corresponding homo-allyl alcohol 9b in 75\% yield with $\mathrm{dr}=>95: 5(27.1 \mathrm{mg}, 0.0765$ mmol, colorless oil).

${ }^{1} \mathrm{H}$ NMR (600 MHz, $\left.\mathrm{CDCl}_{3}, \delta\right): 1.08-1.44(\mathrm{~m}, 6 \mathrm{H}), 1.16(\mathrm{~s}, 3 \mathrm{H}), 1.46-1.61(\mathrm{~m}, 3 \mathrm{H}), 1.62-1.92(\mathrm{~m}$, $8 \mathrm{H}), 2.02-2.10(\mathrm{~m}, 1 \mathrm{H}), 4.88-4.98(\mathrm{~m}, 3 \mathrm{H}), 7.19-7.31$ (m, 5H). ${ }^{13} \mathrm{C} \mathrm{NMR}\left(99 \mathrm{MHz}, \mathrm{CDCl}_{3}, \delta\right): 15.4$ $\left(\mathrm{CH}_{3}\right), 20.1\left(\mathrm{CH}_{2}\right), 26.9\left(\mathrm{CH}_{2}\right), 27.19\left(\mathrm{CH}_{2}\right), 27.22\left(\mathrm{CH}_{2}\right), 28.7\left(\mathrm{CH}_{2}\right), 29.0\left(\mathrm{CH}_{2}\right), 32.2\left(\mathrm{CH}_{2}\right), 33.5$ (q, $\left.J=28.6 \mathrm{~Hz}, \mathrm{CH}_{2}\right), 44.3(\mathrm{CH}), 51.3(\mathrm{C}), 77.0(\mathrm{CH}), 112.6\left(\mathrm{CH}_{2}\right), 127.1$ (q, $\left.J=277.6 \mathrm{~Hz}, C\right), 127.2$ $(\mathrm{CH}), 127.4(\mathrm{CH}), 127.5(\mathrm{CH}), 142.6(\mathrm{C}), 149.8(\mathrm{C})$. HRMS-ESI $(\mathrm{m} / \mathrm{z})$ : $[\mathrm{M}-\mathrm{H}]^{-}$calcd for $\mathrm{C}_{21} \mathrm{H}_{28} \mathrm{OF}_{3}$, 353.2098; found, 353.2099. 


\subsection{Synthesis of 1-(2-bromo-4-fluorophenyl)-2-cyclohexyl-2-methyl-3-methylenepentan-1-ol}

(9c).

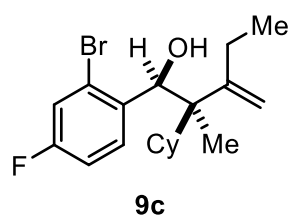

The allylboration reaction was conducted with $58.6 \mathrm{mg}(0.200 \mathrm{mmol})$ of $(E)-4 \mathbf{a b}$ according to the procedure described above. The product $9 \mathbf{c}$ was obtained in 60\% yield with $\mathrm{dr}=>95: 5(44.5 \mathrm{mg}, 0.120$ mmol, colorless oil).

${ }^{1} \mathrm{H}$ NMR (399 MHz, $\left.\mathrm{CDCl}_{3}, \delta\right): 0.67(\mathrm{t}, J=7.3 \mathrm{~Hz}, 3 \mathrm{H}), 1.01(\mathrm{qd}, J=12.3,3.3 \mathrm{~Hz}, 1 \mathrm{H}), 1.11-1.40$ (m, 4H), $1.28(\mathrm{~s}, 3 \mathrm{H}), 1.53-1.60(\mathrm{~m}, 1 \mathrm{H}), 1.63-1.78(\mathrm{~m}, 6 \mathrm{H}), 1.82-1.89(\mathrm{~m}, 1 \mathrm{H}), 2.08-2.16(\mathrm{~m}, 1 \mathrm{H})$, $4.75(\mathrm{~s}, 1 \mathrm{H}), 4.91(\mathrm{~s}, 1 \mathrm{H}), 5.26$ (d, $J=4.1 \mathrm{~Hz}, 1 \mathrm{H}), 6.95$ (ddd, $J=9.6,6.9,1.8 \mathrm{~Hz}, 1 \mathrm{H}), 7.22$ (dd, $J=$ 8.2, $2.7 \mathrm{~Hz}, 1 \mathrm{H}), 7.56(\mathrm{dd}, J=8.7,6.4 \mathrm{~Hz}, 1 \mathrm{H}) .{ }^{13} \mathrm{C} \mathrm{NMR}\left(100 \mathrm{MHz}, \mathrm{CDCl}_{3}, \delta\right): 12.2\left(\mathrm{CH}_{3}\right), 13.7$ $\left(\mathrm{CH}_{3}\right), 25.0\left(\mathrm{CH}_{2}\right), 26.8\left(\mathrm{CH}_{2}\right), 27.3\left(\mathrm{CH}_{2}\right), 27.5\left(\mathrm{CH}_{2}\right), 28.4\left(\mathrm{CH}_{2}\right), 28.8\left(\mathrm{CH}_{2}\right), 46.8(\mathrm{CH}), 51.5(\mathrm{C})$, $76.6(\mathrm{CH}), 110.3\left(\mathrm{CH}_{2}\right), 114.3(\mathrm{~d}, J=20.1 \mathrm{~Hz}, \mathrm{CH}), 119.5(\mathrm{~d}, J=24.0 \mathrm{~Hz}, C \mathrm{H}), 124.1(\mathrm{~d}, J=8.6 \mathrm{~Hz}$, C), 130.9 (d, $J=8.6 \mathrm{~Hz}, C \mathrm{H}), 138.6$ (d, $J=3.8 \mathrm{~Hz}, C$ ), $153.2(C), 161.1$ (d, $J=251.1 \mathrm{~Hz}, C$ ). HRMSESI $(m / z)$ : $[\mathrm{M}-\mathrm{H}]^{-}$calcd for $\mathrm{C}_{19} \mathrm{H}_{25} \mathrm{OBrF}, 367.1078$; found, 367.1082 .

\subsection{Synthesis of 2-cyclohexyl-2-methyl-3-methylene-1-(naphthalen-2-yl)pentan-1-ol (9d).}

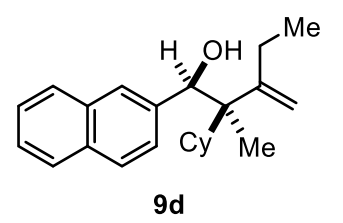

The allylboration reaction was conducted with $120.8 \mathrm{mg}(0.413 \mathrm{mmol})$ of $(E)-\mathbf{4 a b}$ according to the procedure described above. The product 9d was obtained in 52\% yield with $\mathrm{dr}=>95: 5(69.7 \mathrm{mg}, 0.216$ mmol, white solid).

${ }^{1} \mathrm{H}$ NMR $\left(392 \mathrm{MHz}, \mathrm{CDCl}_{3}, \delta\right): 0.81$ (t, $\left.J=7.1 \mathrm{~Hz}, 3 \mathrm{H}\right), 1.10-1.42(\mathrm{~m}, 5 \mathrm{H}), 1.19$ (s, 3H), 1.51$1.81(\mathrm{~m}, 6 \mathrm{H}), 1.82-1.93(\mathrm{~m}, 1 \mathrm{H}), 2.01-2.21(\mathrm{~m}, 2 \mathrm{H}), 4.88(\mathrm{~s}, 1 \mathrm{H}), 4.96(\mathrm{~s}, 1 \mathrm{H}), 5.12(\mathrm{~s}, 1 \mathrm{H}), 7.39-$ 7.49 (m, 3H), 7.69-7.76 (m, 2H), 7.76-7.84 (m, 2H). $\left.{ }^{13} \mathrm{C} \mathrm{NMR} \mathrm{(99} \mathrm{MHz,} \mathrm{CDCl}_{3}, \delta\right): 12.1\left(\mathrm{CH}_{3}\right), 15.8$ $\left(\mathrm{CH}_{3}\right), 25.8\left(\mathrm{CH}_{2}\right), 27.0\left(\mathrm{CH}_{2}\right), 27.2\left(\mathrm{CH}_{2}\right), 27.3\left(\mathrm{CH}_{2}\right), 28.8\left(\mathrm{CH}_{2}\right), 29.0\left(\mathrm{CH}_{2}\right), 44.2(\mathrm{CH}), 51.6(\mathrm{C})$, $76.7(\mathrm{CH}), 111.4\left(\mathrm{CH}_{2}\right), 125.5(\mathrm{CH}), 125.7(\mathrm{CH}), 125.9(\mathrm{CH}), 126.1(\mathrm{CH}), 126.5(\mathrm{CH}), 127.4(\mathrm{CH})$, $128.0(\mathrm{CH}), 132.5(\mathrm{C}), 132.6(\mathrm{C}), 140.4(\mathrm{C}), 152.5(\mathrm{C})$. HRMS-ESI $(\mathrm{m} / \mathrm{z})$ : $[\mathrm{M}+\mathrm{Na}]^{+}$calcd for $\mathrm{C}_{23} \mathrm{H}_{30} \mathrm{ONa}$, 345.2189; found, 345.2191 . 
7.5. Synthesis of $\{(8 S, 9 S, 13 S, 14 S, 17 S)-3$-methoxy-13-methyl-17-(6,6,6-trifluorohex-1-en-2-yl)7,8,9,11,12,13,14,15,16,17-decahydro-6H-cyclopenta[a]phenanthren-17-yl\}methanol (9e).

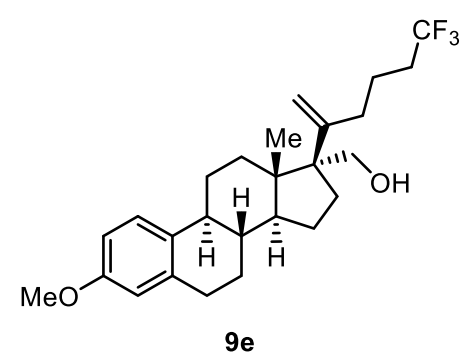

The allylboration reaction was conducted with $240.6 \mathrm{mg}(0.452 \mathrm{mmol})$ of $(E)-4 \mathrm{ma}$ according to the procedure described in section 7.1. The product 9e was obtained in $84 \%$ yield with $\mathrm{dr}=>95: 5$ (165.1 mg, $0.378 \mathrm{mmol}$, white solid).

${ }^{1} \mathrm{H}$ NMR $\left(392 \mathrm{MHz}, \mathrm{CDCl}_{3}, \delta\right): 0.72$ (s, 3H), 1.18-1.58 (m, 5H), 1.59-2.03 (m, 9H), 2.04-2.40 (m, $6 \mathrm{H}), 2.76-2.95(\mathrm{~m}, 2 \mathrm{H}), 3.45(\mathrm{~d}, J=10.2 \mathrm{~Hz}, 1 \mathrm{H}), 3.71(\mathrm{~s}, 3 \mathrm{H}), 3.71-3.85(\mathrm{~m}, 1 \mathrm{H}), 5.09(\mathrm{~d}, J=18.8$ $\mathrm{Hz}, 2 \mathrm{H}), 6.62(\mathrm{~d}, J=2.7 \mathrm{~Hz}, 1 \mathrm{H}), 6.69(\mathrm{dd}, J=8.6 \mathrm{~Hz}, 2.7 \mathrm{~Hz}, 1 \mathrm{H}), 7.16(\mathrm{~d}, J=8.6 \mathrm{~Hz}, 1 \mathrm{H}) .{ }^{13} \mathrm{C}$ NMR (99 MHz, $\left.\mathrm{CDCl}_{3}, \delta\right): 15.7\left(\mathrm{CH}_{3}\right), 20.5\left(\mathrm{CH}_{2}\right), 23.4\left(\mathrm{CH}_{2}\right), 26.3\left(\mathrm{CH}_{2}\right), 27.7\left(\mathrm{CH}_{2}\right), 27.8\left(\mathrm{CH}_{2}\right)$, $29.7\left(\mathrm{CH}_{2}\right), 32.4\left(\mathrm{br}, \mathrm{CH}_{2}\right), 33.1\left(\mathrm{CH}_{2}\right), 33.4\left(\mathrm{q}, J=28.6 \mathrm{~Hz}, \mathrm{CH}_{2}\right), 39.2(\mathrm{CH}), 43.3(\mathrm{CH}), 45.9(\mathrm{C})$, $50.9(\mathrm{CH}), 55.0\left(\mathrm{CH}_{3}\right), 59.5(\mathrm{C}), 63.7\left(\mathrm{CH}_{2}\right), 111.3(\mathrm{CH}), 112.9\left(\mathrm{CH}_{2}\right), 113.6(\mathrm{CH}), 126.0(\mathrm{CH}), 127.0$ (q, $J=277.6 \mathrm{~Hz}, C), 132.3(C), 137.8(C), 150.6(C), 157.3(C)$. HRMS-EI $(m / z)$ : [M] $]^{+}$calcd for $\mathrm{C}_{26} \mathrm{H}_{35} \mathrm{~F}_{3} \mathrm{O}_{2}, 436.2589$; found, 436.2582 . 


\section{Evaluation of Accuracy of ${ }^{13}$ C NMR Analysis for Stereoselectivity Determination}

During the optimization of reaction conditions (Table 1), the stereoselectivity of allyl boron compound 4aa was determined by ${ }^{13} \mathrm{C}$ NMR analysis because the other analyses, including a quantitative ${ }^{1} \mathrm{H}$ and ${ }^{19} \mathrm{~F}$ NMR, GC, HPLC analysis, were unfortunately not effective for determining the stereoselectivity of $4 \mathbf{a a}$ due to lack of the peak separation between the regio- and stereoisomers. As the accuracy of ${ }^{13} \mathrm{C}$ NMR analysis is not thoroughly investigated, ${ }^{14}$ we evaluated the accuracy of ${ }^{13} \mathrm{C}$ NMR for determining the stereoselectivity of $4 \mathbf{a a}$ by comparing the selectivity determined by ${ }^{13} \mathrm{C}$ NMR analysis of 4aa against that determined by quantitative- ${ }^{1} \mathrm{H}$ NMR (q- ${ }^{1} \mathrm{H}$ NMR) analysis of corresponding oxidation product 6aa obtained via stereospecific oxidation reaction of 4aa (Figure S1). For this purpose, we chose Xantphos, IMes, and IAd ligand (Table 1, entries 1, 6, and 7, respectively), because the reactions with those ligands produced 4aa with high regioselectivity $(\mathbf{4 a a} / \mathbf{5 a a}=>94: 6)$. Then, we found a perfect linear correlation between $(E)$-selectivity of 4aa and $(Z)$-selectivity of $6 \mathbf{a a}$ (Figure S2). Thus, we could confirm that the accuracy of ${ }^{13} \mathrm{C}$ NMR analysis is the same as that of quantitative- ${ }^{1} \mathrm{H}$ NMR analysis, and we assured the accuracy of the stereoselectivity of 4aa described in Table1.

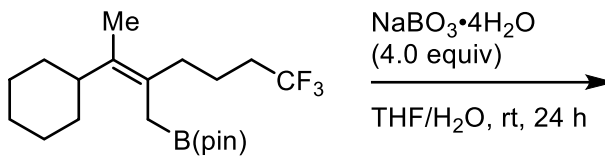

$(E)-4 a a$

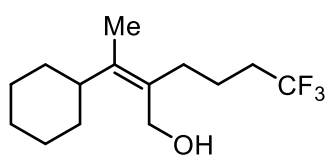

(Z)-6aa

Figure S1. Method for evaluation of accuracy of ${ }^{13} \mathrm{C}$ NMR analysis. 


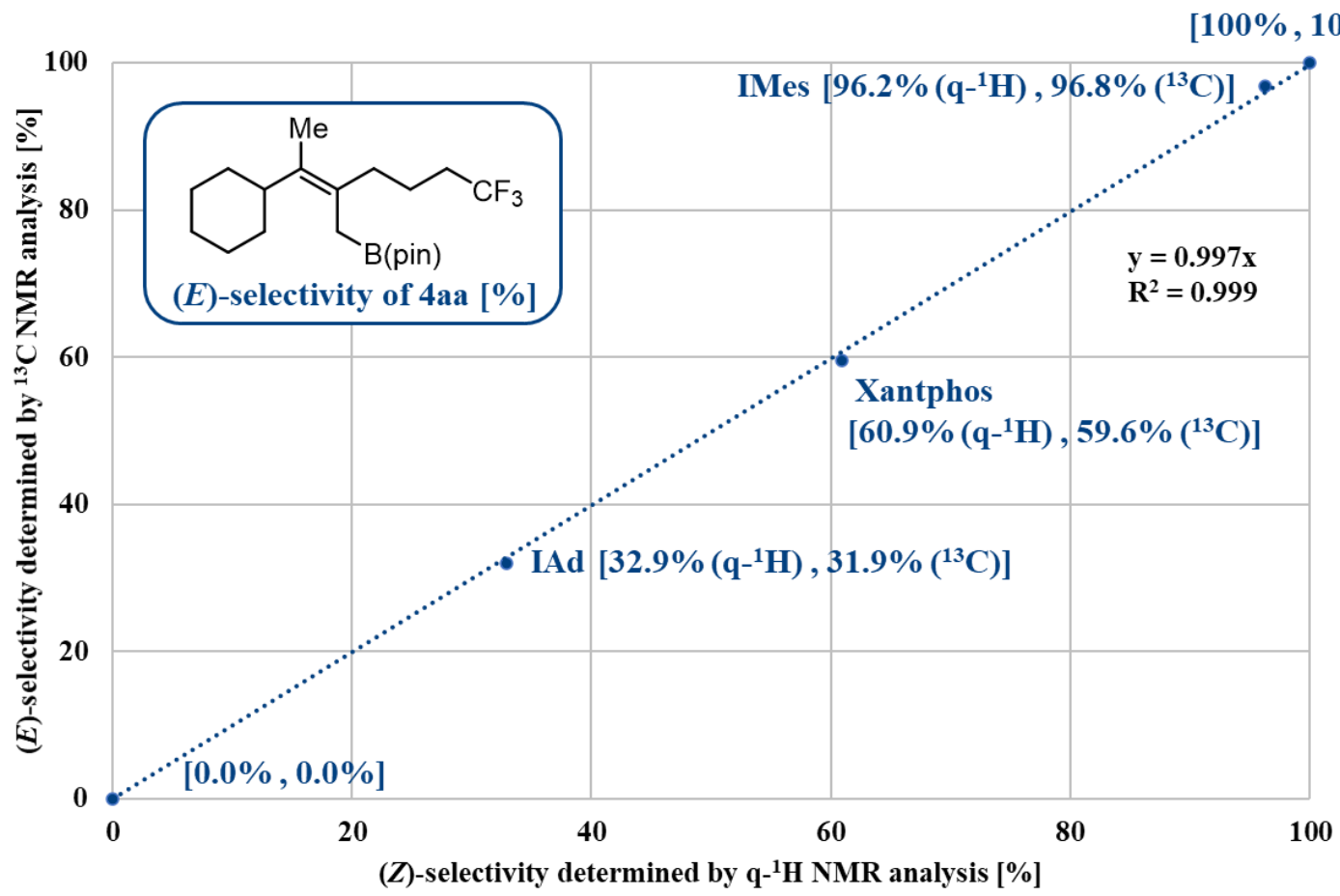

Figure S2. Relationship between $(E)$-selectivity of 4aa and (Z)-selectivity of 6aa. 


\section{2D NMR Study for Determination of Product Stereochemistry}

\subsection{Stereochemistry of (E)-2-(2-(1-cyclohexylethylidene)-6,6,6-trifluorohexyl)-}

4,4,5,5-tetramethyl-1,3,2-dioxaborolane $[(E)-4 a a]$.

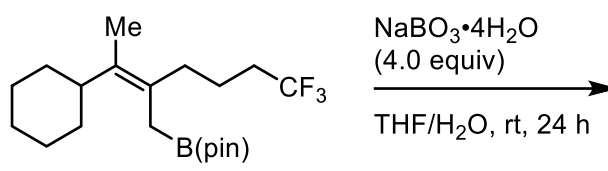

(E)-4aa<smiles>CC(=C(CO)CCCC(F)(F)F)C1CCCCC1</smiles>

(Z)-6aa

The stereochemistry of (E)-4aa was determined by ${ }^{1} \mathrm{H}$ NMR NOESY experiment after the stereospecific oxidation of the boryl group in $(E)$-4aa. After the oxidation reaction of $(E)-\mathbf{4 a a}$, the crude material was purified by silica gel column chromatography to give the (Z)-6aa as a single isomer $(E / Z=<5: 95)$. The results were summarized as the following schemes (Figures S3 and S4). A solid curved arrow indicates the selected nOe around the alkene moiety.

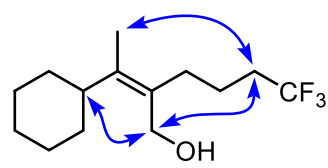

Figure S3. Summary of NOESY analysis of the alcohol product (Z)-6aa. 


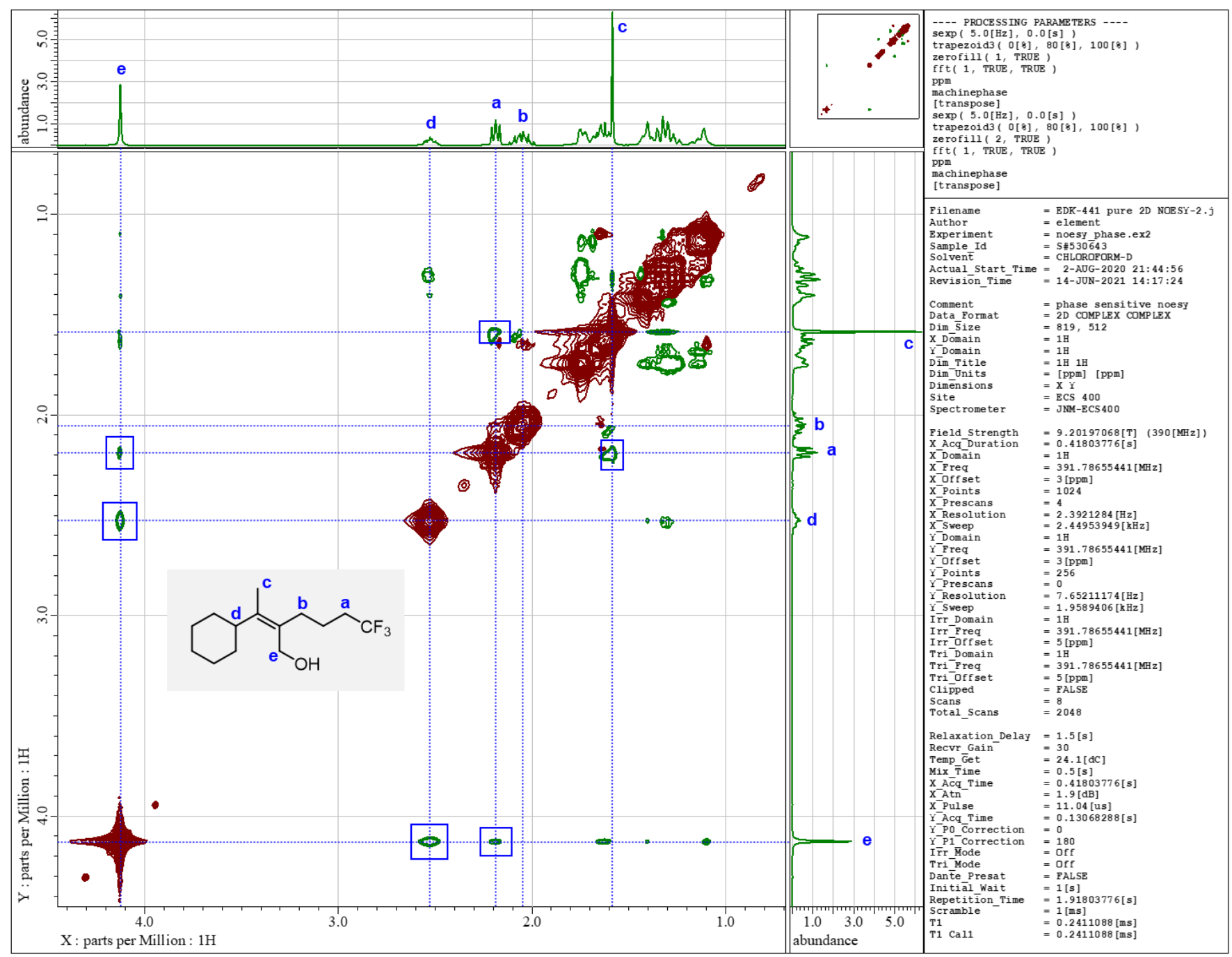

Figure S4. NOESY spectrum of the alcohol product (Z)-6aa and the selected correlation peaks 


\subsection{Stereochemistry of (Z)-2-(2-(1-cyclohexylethylidene)-6,6,6-trifluorohexyl)-}

\section{4,4,5,5-tetramethyl-1,3,2-dioxaborolane $[(Z)-4 a a]$.}<smiles>CC(=C(CBr)CCCC(F)(F)F)C1CCCCC1</smiles>

(Z)-4aa

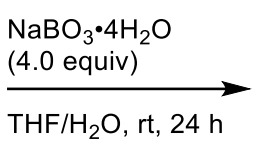

$\mathrm{THF} / \mathrm{H}_{2} \mathrm{O}, \mathrm{rt}, 24 \mathrm{~h}$<smiles>CC(CO)=C(CCCC(F)(F)F)C1CCCCC1</smiles>

(E)-6aa

The stereochemistry of (Z)-4aa was determined by a ${ }^{1} \mathrm{H}$ NMR NOESY experiment after the stereospecific oxidation of the boryl group in (Z)-4aa. (Z)-4aa was prepared via the borylation reaction using IAdCuCl as catalyst (Table 1, entry 7). After the oxidation reaction of $(Z)-\mathbf{4 a a}$, the crude material was purified by silica gel column chromatography to give the $(E)$-6aa as a single isomer $(E / Z=>95: 5)$. The results were summarized as the following schemes (Figures S5 and S6). A solid curved arrow indicates the selected nOe around the alkene moiety.

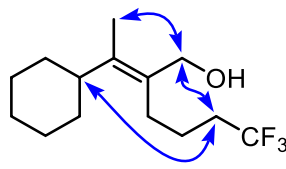

Figure S5. Summary of NOESY analysis of the alcohol product (E)-6aa. 


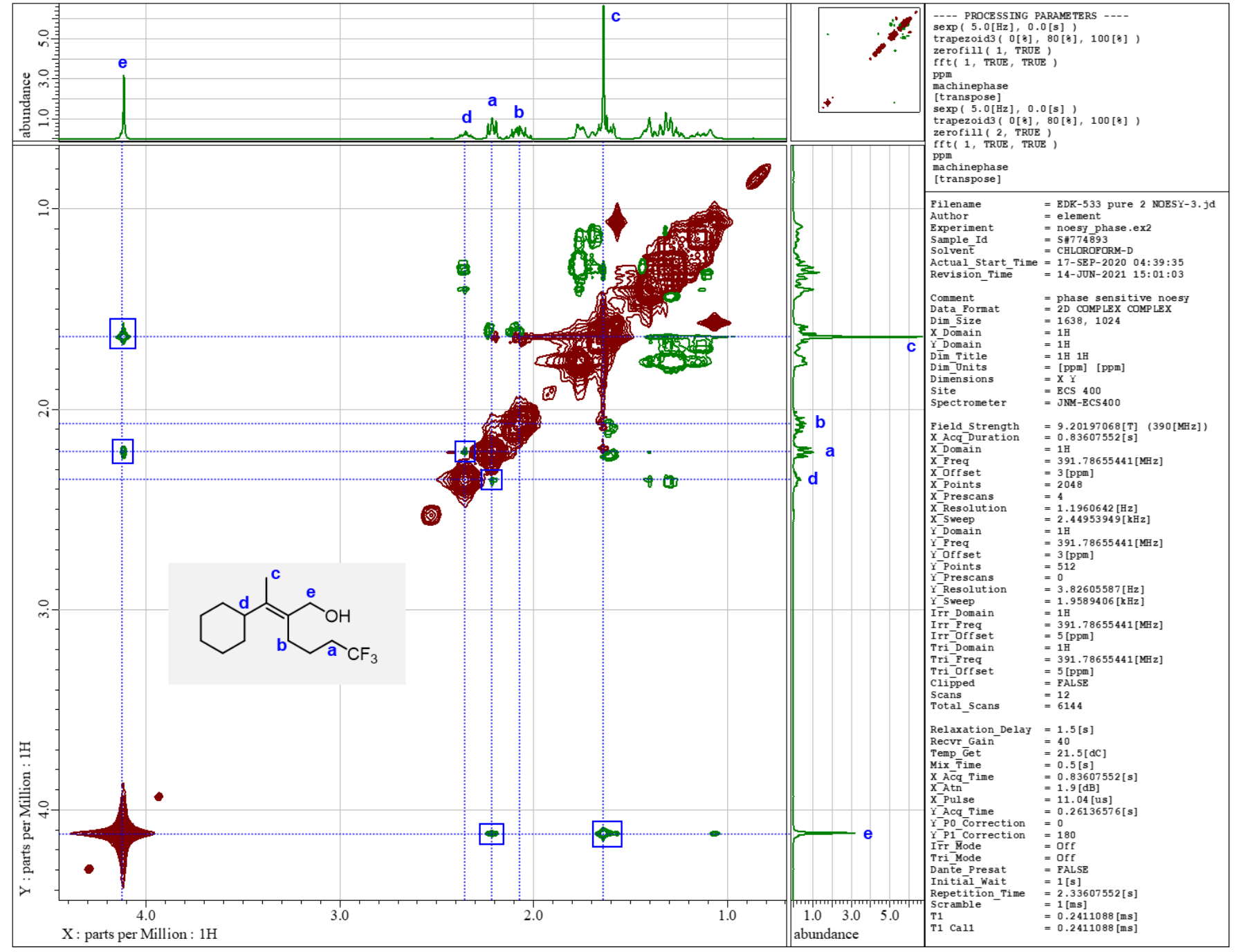

Figure S6. NOESY spectrum of the alcohol product $(E)$-6aa and the selected correlation peaks 


\section{Alkylboration of Aryl-substituted Allene.}

The optimized conditions for gem-dialkylallenes were applied to an aryl-substituted allene 1n (Figure S7). Under Conditions A, the reaction afforded a complex mixture (Figure S7A). Allylic boronate $\mathbf{4 n b}$ and alkenyl boronate $\mathbf{5 n b}$ were found in the mixture, albeit with no regioselectivity $(\mathbf{4} / \mathbf{5}$ $=51: 49)$. Conversely, 4nb was obtained as the major product under Conditions $\mathbf{B}$, although the regioselectivity was not sufficient (Figure S7B). Thus, we conclude that the optimized conditions for gem-dialkylallenes are not suitable for aryl-substituted allenes.

A. SIMes ligand (Conditions A)

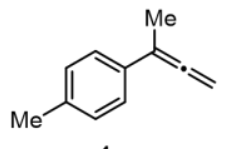

$1 n$

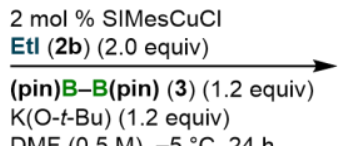

DMF $(0.5 \mathrm{M}),-5^{\circ} \mathrm{C}, 24 \mathrm{~h}$

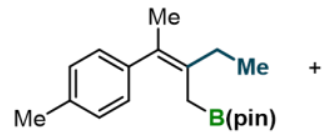

$4 \mathrm{nb}$

complex mixture $\mathbf{4 / 5}=51: 49$

B. Xantphos ligand (Conditions B)

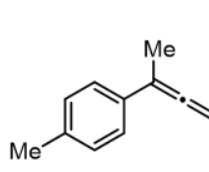

1n

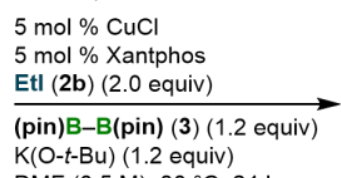

$5 \mathrm{~mol} \% \mathrm{CuCl}$

$5 \mathrm{~mol} \%$ Xantphos

Etl (2b) (2.0 equiv)

(pin)B-B(pin) (3) (1.2 equiv) $\mathrm{K}(\mathrm{O}-t-\mathrm{Bu})$ (1.2 equiv) $\operatorname{DMF}(0.5 \mathrm{M}), 30^{\circ} \mathrm{C}, 24 \mathrm{~h}$

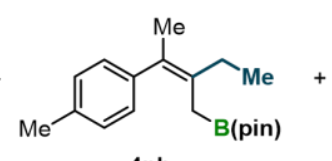

4nb $64 \%$ NMR yield

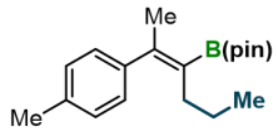

$5 \mathrm{nb}$

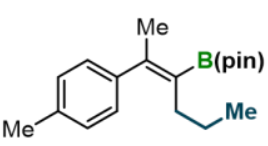

$5 \mathrm{nb}$ $16 \%$ NMR yield

$4 / 5=80: 20$

Figure S7. Alkylboration of aryl-substituted allene $\mathbf{1 n}$ using Conditions $\mathbf{A}$ and $\mathbf{B}$. 


\section{Stoichiometric Reaction.}

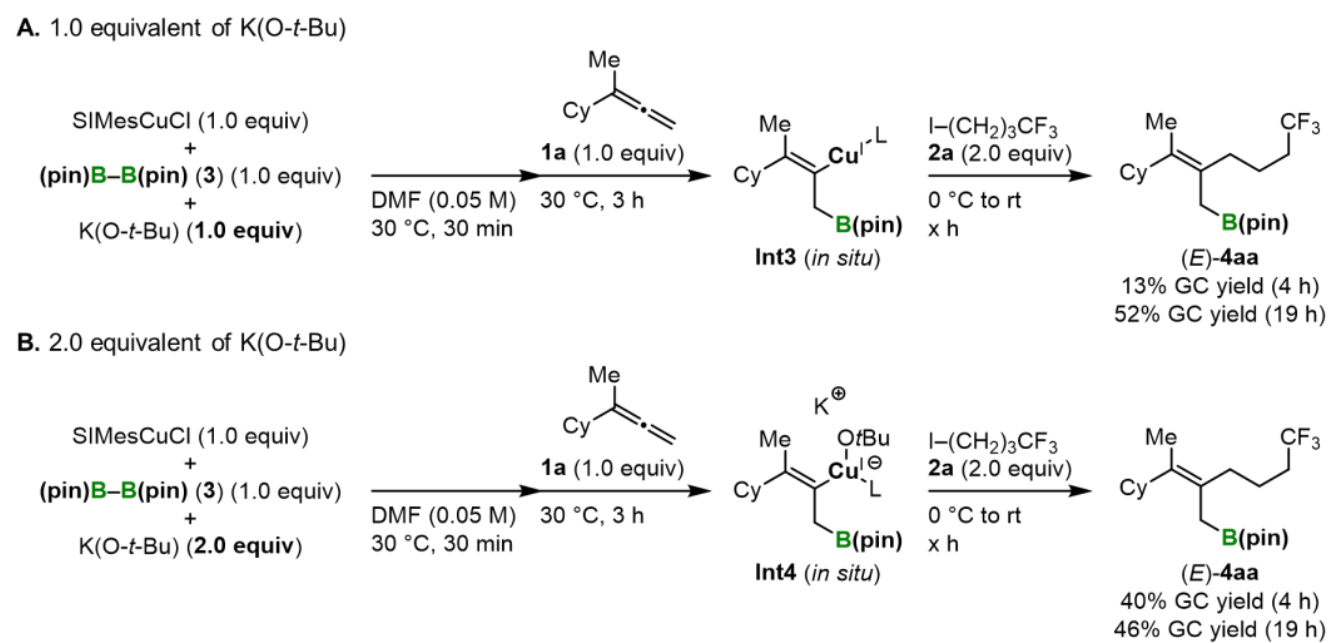

Figure S8. Stoichiometric reactions using 1.0 and 2.0 equivalents of the alkoxide base.

SIMesCuCl (40.5 mg, $0.10 \mathrm{mmol})$, bis(pinacolato)diboron (3) $(25.4 \mathrm{mg}, 0.10 \mathrm{mmol})$, and $\mathrm{K}(\mathrm{O}-\mathrm{t}$ $\mathrm{Bu}$ ) (for Figure S8A; $11.2 \mathrm{mg}, 0.10 \mathrm{mmol}$, for Figure S8B; $22.4 \mathrm{mg}, 0.10 \mathrm{mmol}$ ) were placed in an oven-dried reaction vial in an argon-filled glove box. After the vial was sealed with a screw cap containing a Teflon ${ }^{\mathrm{TM}}$-coated rubber septum and taken from the glove box, dry DMF $(2.0 \mathrm{~mL})$ was added to the vial through the rubber septum using a syringe. After stirring at $30^{\circ} \mathrm{C}$ for $30 \mathrm{~min}$, allene $1 \mathrm{a}(13.6 \mathrm{mg}, 0.10 \mathrm{mmol})$ was added to the mixture. The reaction mixture was stirred at $30^{\circ} \mathrm{C}$ for $3 \mathrm{~h}$. Then, alkyl halide $2 \mathrm{a}(47.6 \mathrm{mg}, 0.20 \mathrm{mmol})$ was added to the mixture at $0{ }^{\circ} \mathrm{C}$, and the mixture was allowed to warm to room temperature. The reaction monitoring was done by GC analysis using 1,4diisopropylbenzene was internal standard.

To confirm the generation of cuprate species Int4 in the catalytic cycle (Figure 6A), we performed stoichiometric reactions as described above (Figure S8). The reaction between the SIMesCuCl complex and bis(pinacolato)diboron (3) in the presence of 1.0 equivalent of $\mathrm{K}(\mathrm{O}-\mathrm{t}-\mathrm{Bu})$, followed by the addition of allene 1a can be expected to form borylcopper(I) species Int3 in situ (Figure S8A). After stirring the mixture for $3 \mathrm{~h}$, the consumption of allene 1a was $66 \%$. The following reaction between Int3 and alkyl halide 2a gave $(E)$-4aa in low yield at the initial stage of the reaction $(4 \mathrm{~h}$ : $13 \% \mathrm{GC}$ yield). Thereafter, the yield reached moderate levels ( $19 \mathrm{~h}: 52 \% \mathrm{GC}$ yield). On the other hand, the reaction with 2.0 equivalent of $\mathrm{K}(\mathrm{O}-t-\mathrm{Bu})$ can be expected to form cuprate species Int4 (Figure S8B). After stirring the mixture for $3 \mathrm{~h}$, the consumption of allene $1 \mathrm{a}$ was $65 \%$. After the adding $\mathbf{2 a}$, (E)-4aa was immediately generated in moderate yield ( $4 \mathrm{~h}: 40 \% \mathrm{GC}$ yield). Finally, the yield increased to almost the same level as that with 1.0 equivalent of the base ( $19 \mathrm{~h}$ : $46 \% \mathrm{GC}$ yield). These results indicate that the consumption of the allene is not affected by the amount of alkoxide base. In contrast, an excess of alkoxide can be expected to promote the alkylation step to produce the alkylboration 
product $(E)$-4aa faster in the initial stage of the reaction. However, further studies are required to propose a conclusive mechanism for the alkylation step in situ. 


\section{Single Crystal X-ray Structural Analysis}

12.1. Molecular structure and $X$-ray crystallographic data of $(E)$-4ga.

The stereochemistry of $(E)$-4ga was determined by X-ray crystallographic analysis. The details were summarized in Figure S9 and Table S1.
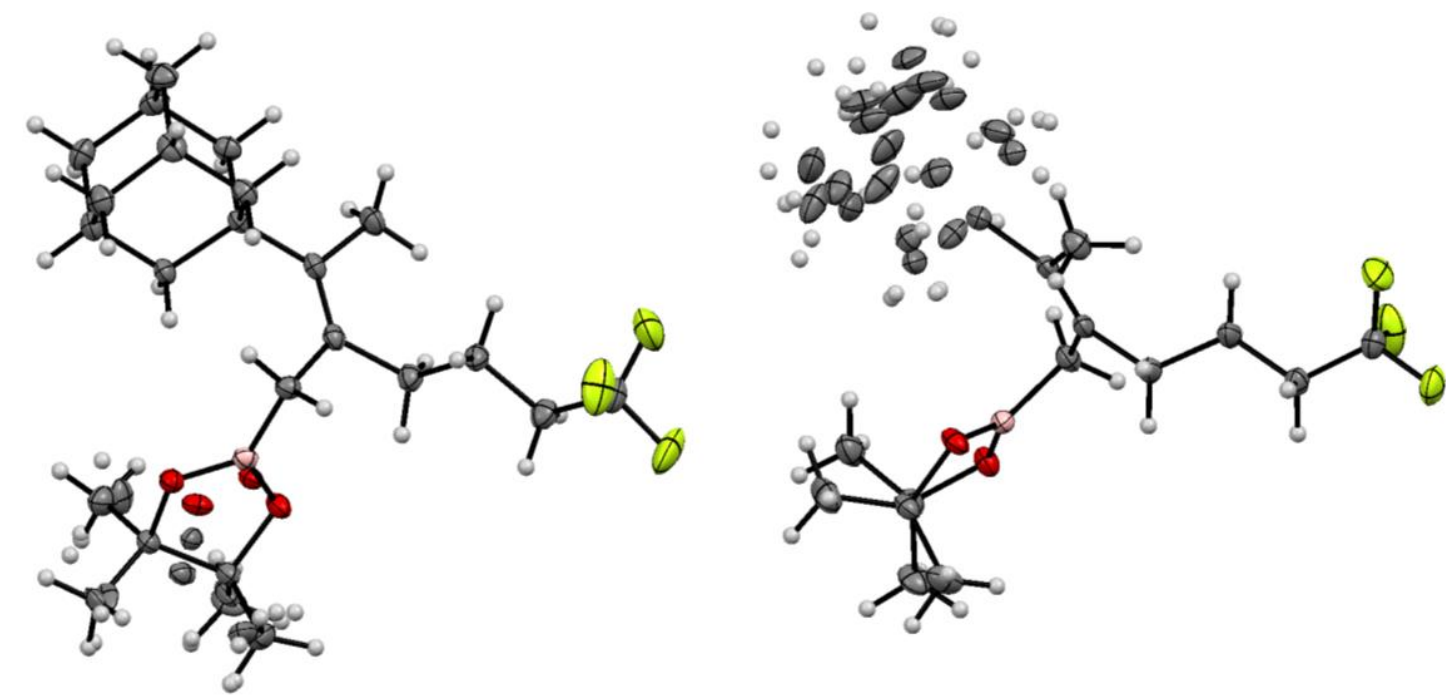

Figure S9. Molecular structure of $(E)$-4ga. 
Table S1. Summary of X-ray crystallographic data of (E)-4ga.

\begin{tabular}{|c|c|}
\hline CCDC Name & 2062673 \\
\hline Empirical Formula & $\mathrm{C}_{24} \mathrm{H}_{38} \mathrm{BF}_{3} \mathrm{O}_{2}$ \\
\hline Formula Weight & 426.35 \\
\hline Crystal System & monoclinic \\
\hline Crystal Size / mm & $0.100 \times 0.100 \times 0.100$ \\
\hline$a / \AA$ & $11.5507(2)$ \\
\hline$b / \AA$ & $10.4530(2)$ \\
\hline$c / \AA$ & $38.0880(8)$ \\
\hline$\alpha /^{\circ}$ & 90.0000 \\
\hline$\beta /^{\circ}$ & $90.206(2)$ \\
\hline$\gamma /{ }^{\circ}$ & 90.0000 \\
\hline$V / \AA^{3}$ & $4598.70(15)$ \\
\hline Space Group & $P 2_{1} / \mathrm{n}$ \\
\hline$Z$ value & 8 \\
\hline Dcalc $/ \mathrm{g} \mathrm{cm}^{-3}$ & 1.232 \\
\hline Temperature / K & 123 \\
\hline $2 \theta_{\max } /^{\circ}$ & 58.84 \\
\hline$\mu(\mathrm{CuK} \alpha) / \mathrm{mm}^{-1}$ & - \\
\hline$\mu(\operatorname{MoK} \alpha) / \mathrm{mm}^{-1}$ & 0.091 \\
\hline $\begin{array}{l}\text { No. of Reflections } \\
\text { Measured }\end{array}$ & $\begin{array}{l}\text { Total: } 60487 \\
\text { Unique: } 11431 \\
\left(R_{\text {int }}=0.0402\right)\end{array}$ \\
\hline $\begin{array}{l}\text { No. of Observations } \\
\text { (All reflections) }\end{array}$ & 11431 \\
\hline $\begin{array}{l}\text { Residuals: } R_{1} \\
(\mathrm{I}>2.00 \sigma(\mathrm{I}))\end{array}$ & 0.0579 \\
\hline $\begin{array}{l}\text { Residuals: } w R_{2} \\
\text { (All reflections) }\end{array}$ & 0.1396 \\
\hline $\begin{array}{l}\text { Goodness of Fit Indicator } \\
\text { (GOF) }\end{array}$ & 1.126 \\
\hline $\begin{array}{l}\text { Maximum Peak in } \\
\text { Final Diff. Map / } \AA^{3}\end{array}$ & 0.31 \\
\hline $\begin{array}{l}\text { Minimum Peak in } \\
\text { Final Diff. Map / } \AA^{3}\end{array}$ & -0.32 \\
\hline Flack parameter & - \\
\hline
\end{tabular}


12.2. Molecular structure and $X$-ray crystallographic data of $9 \mathrm{~d}$.

The stereochemistry of $9 \mathbf{d}$ was determined by X-ray crystallographic analysis. The details were summarized in Figure S10 and Table S2.

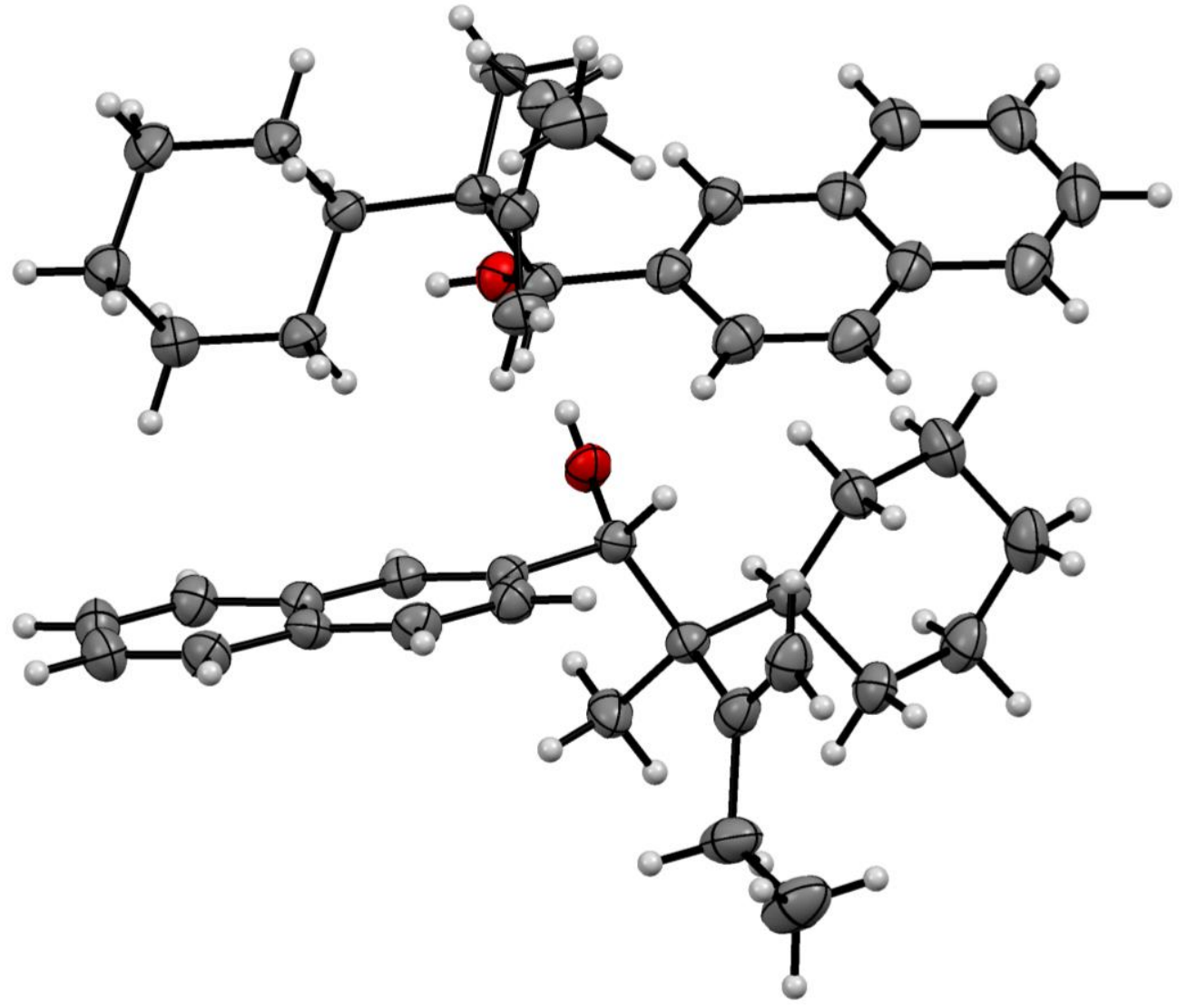

Figure S10. Molecular structure of 9d. 
Table S2. Summary of X-ray crystallographic data of 9d.

\begin{tabular}{|c|c|}
\hline CCDC Name & 2067460 \\
\hline Empirical Formula & $\mathrm{C}_{23} \mathrm{H}_{30} \mathrm{O}$ \\
\hline Formula Weight & 322.47 \\
\hline Crystal System & monoclinic \\
\hline Crystal Size / mm & $0.200 \times 0.100 \times 0.100$ \\
\hline$a / \AA$ & $10.56870(10)$ \\
\hline$b / \AA$ & $15.6326(2)$ \\
\hline$c / \AA$ & $22.9525(3)$ \\
\hline$\alpha /^{\circ}$ & 90.0000 \\
\hline$\beta /^{\circ}$ & $101.9420(10)$ \\
\hline$\gamma /{ }^{\circ}$ & 90.0000 \\
\hline$V / \AA^{3}$ & $3710.05(8)$ \\
\hline Space Group & $P 2{ }_{1} / \mathrm{c}$ \\
\hline$Z$ value & 8 \\
\hline Dcalc $/ \mathrm{g} \mathrm{cm}^{-3}$ & 1.155 \\
\hline Temperature / K & 123 \\
\hline $2 \theta_{\max } /^{\circ}$ & 150.372 \\
\hline$\mu(\mathrm{CuK} \alpha) / \mathrm{mm}^{-1}$ & 0.516 \\
\hline$\mu(\operatorname{MoK} \alpha) / \mathrm{mm}^{-1}$ & - \\
\hline $\begin{array}{l}\text { No. of Reflections } \\
\text { Measured }\end{array}$ & $\begin{array}{l}\text { Total: } 18532 \\
\text { Unique: } 18532 \\
\left(R_{\text {int }}=\mathrm{N} / \mathrm{A}^{a}\right)\end{array}$ \\
\hline $\begin{array}{l}\text { No. of Observations } \\
\text { (All reflections) }\end{array}$ & 18532 \\
\hline $\begin{array}{l}\text { Residuals: } R_{1} \\
(\mathrm{I}>2.00 \sigma(\mathrm{I}))\end{array}$ & 0.0708 \\
\hline $\begin{array}{l}\text { Residuals: } w R_{2} \\
\text { (All reflections) }\end{array}$ & 0.2115 \\
\hline $\begin{array}{l}\text { Goodness of Fit Indicator } \\
\text { (GOF) }\end{array}$ & 1.111 \\
\hline $\begin{array}{l}\text { Maximum Peak in } \\
\text { Final Diff. Map / } \AA^{3}\end{array}$ & 0.43 \\
\hline $\begin{array}{l}\text { Minimum Peak in } \\
\text { Final Diff. Map / } \AA^{3}\end{array}$ & -0.31 \\
\hline Flack parameter & - \\
\hline
\end{tabular}

${ }^{a}$ Not available because of the twin analyses. 
12.3. Molecular structure and $X$-ray crystallographic data of $(E)-4 \mathrm{mb}$.

The stereochemistry of $(E)-\mathbf{4 m b}$ was determined by X-ray crystallographic analysis. The details were summarized in Figure S11 and Table S3.

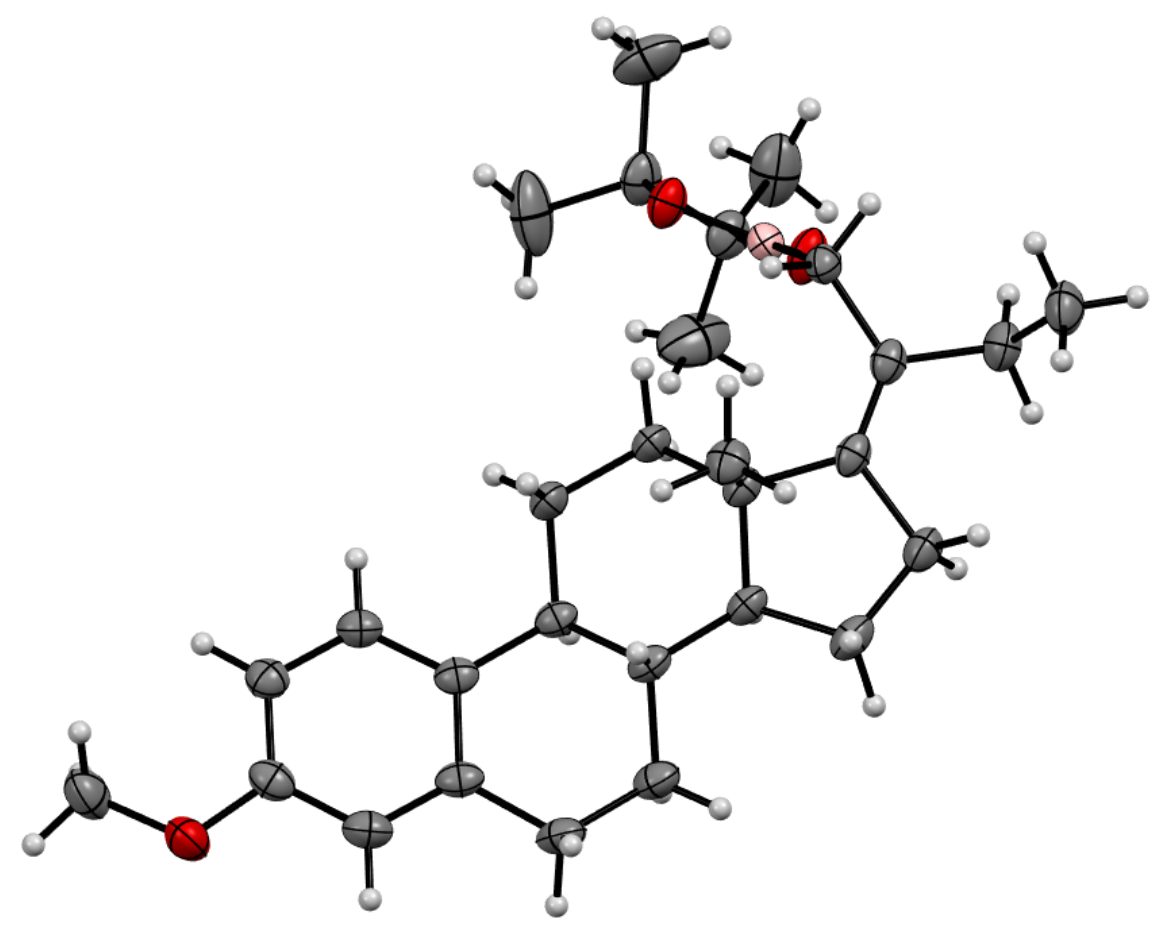

Figure S11. Molecular structure of $(E)-\mathbf{4 m b}$. 
Table S3. Summary of X-ray crystallographic data of $(E)-\mathbf{4 m b}$.

\begin{tabular}{|c|c|}
\hline CCDC Name & 2070912 \\
\hline Empirical Formula & $\mathrm{C}_{29} \mathrm{H}_{43} \mathrm{BO}_{3}$ \\
\hline Formula Weight & 450.44 \\
\hline Crystal System & orthorhombic \\
\hline Crystal Size / mm & $0.200 \times 0.200 \times 0.200$ \\
\hline$a / \AA$ & $7.41170(10)$ \\
\hline$b / \AA$ & $12.02580(10)$ \\
\hline$c / \AA$ & $29.9155(2)$ \\
\hline$\alpha /^{\circ}$ & 90.0000 \\
\hline$\beta /^{\circ}$ & 90.0000 \\
\hline$\gamma /{ }^{\circ}$ & 90.0000 \\
\hline$V / \AA^{3}$ & $2666.42(5)$ \\
\hline Space Group & $P 2{ }_{1}{ }_{1} 2_{1}$ \\
\hline$Z$ value & 4 \\
\hline$D$ calc $/ \mathrm{g} \mathrm{cm}^{-3}$ & 1.122 \\
\hline Temperature / K & 123 \\
\hline $2 \theta_{\max } /^{\circ}$ & 153.09 \\
\hline$\mu(\mathrm{CuK} \alpha) / \mathrm{mm}^{-1}$ & 0.538 \\
\hline$\mu(\mathrm{MoK} \alpha) / \mathrm{mm}^{-1}$ & - \\
\hline $\begin{array}{l}\text { No. of Reflections } \\
\text { Measured }\end{array}$ & $\begin{array}{l}\text { Total: } 42609 \\
\text { Unique: } 5506 \\
\left(R_{\text {int }}=\mathrm{N} / \mathrm{A}^{a}\right)\end{array}$ \\
\hline $\begin{array}{l}\text { No. of Observations } \\
\text { (All reflections) }\end{array}$ & 5506 \\
\hline $\begin{array}{l}\text { Residuals: } R_{1} \\
(\mathrm{I}>2.00 \sigma(\mathrm{I}))\end{array}$ & 0.0407 \\
\hline $\begin{array}{l}\text { Residuals: } w R_{2} \\
\text { (All reflections) }\end{array}$ & 0.1061 \\
\hline $\begin{array}{l}\text { Goodness of Fit Indicator } \\
\text { (GOF) }\end{array}$ & 1.079 \\
\hline $\begin{array}{l}\text { Maximum Peak in } \\
\text { Final Diff. Map / } \AA^{3}\end{array}$ & 0.29 \\
\hline $\begin{array}{l}\text { Minimum Peak in } \\
\text { Final Diff. Map / } \AA^{3}\end{array}$ & -0.22 \\
\hline Flack parameter & $-0.10(7)$ \\
\hline
\end{tabular}




\subsection{Molecular structure and $X$-ray crystallographic data of 9e.}

The stereochemistry of $\mathbf{9 e}$ was determined by X-ray crystallographic analysis. The details were summarized in Figure S12 and Table S4.

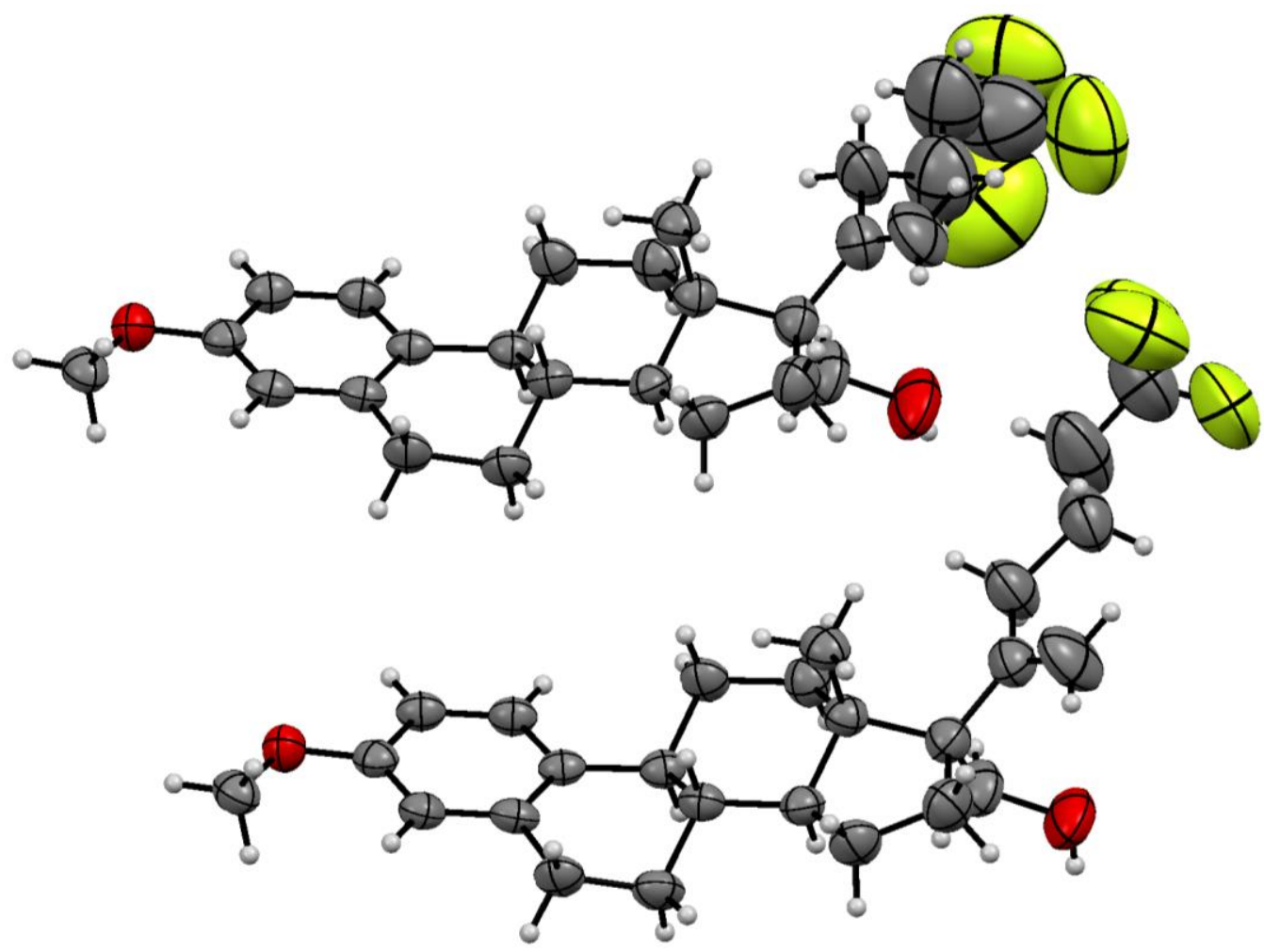

Figure S12. Molecular structure of 9e. 
Table S4. Summary of X-ray crystallographic data of 9e.

\begin{tabular}{|c|c|}
\hline CCDC Name & 2067461 \\
\hline Empirical Formula & $\mathrm{C}_{26} \mathrm{H}_{35} \mathrm{~F}_{3} \mathrm{O}_{2}$ \\
\hline Formula Weight & 436.54 \\
\hline Crystal System & monoclinic \\
\hline Crystal Size / mm & $1.50 \times 1.50 \times 0.200$ \\
\hline$a / \AA$ & $12.6735(2)$ \\
\hline$b / \AA$ & $6.86020(10)$ \\
\hline$c / \AA$ & $26.7831(5)$ \\
\hline$\alpha /^{\circ}$ & 90.0000 \\
\hline$\beta /^{\circ}$ & $97.082(2)$ \\
\hline$\gamma /{ }^{\circ}$ & 90.0000 \\
\hline$V / \AA^{3}$ & $2310.83(7)$ \\
\hline Space Group & $P 2_{1}$ \\
\hline$Z$ value & 4 \\
\hline Dcalc $/ \mathrm{g} \mathrm{cm}^{-3}$ & 1.255 \\
\hline Temperature / K & 293 \\
\hline $2 \theta_{\max } /^{\circ}$ & 150.316 \\
\hline$\mu(\mathrm{CuK} \alpha) / \mathrm{mm}^{-1}$ & 0.772 \\
\hline$\mu(\mathrm{MoK} \alpha) / \mathrm{mm}^{-1}$ & - \\
\hline $\begin{array}{l}\text { No. of Reflections } \\
\text { Measured }\end{array}$ & $\begin{array}{l}\text { Total: } 23607 \\
\text { Unique: } 8055 \\
\left(R_{\text {int }}=0.0427\right)\end{array}$ \\
\hline $\begin{array}{l}\text { No. of Observations } \\
\text { (All reflections) }\end{array}$ & 8055 \\
\hline $\begin{array}{l}\text { Residuals: } R_{1} \\
(\mathrm{I}>2.00 \sigma(\mathrm{I}))\end{array}$ & 0.0920 \\
\hline $\begin{array}{l}\text { Residuals: } w R_{2} \\
\text { (All reflections) }\end{array}$ & 0.3124 \\
\hline $\begin{array}{l}\text { Goodness of Fit Indicator } \\
\text { (GOF) }\end{array}$ & 1.221 \\
\hline $\begin{array}{l}\text { Maximum Peak in } \\
\text { Final Diff. Map / } \AA^{3}\end{array}$ & 0.58 \\
\hline $\begin{array}{l}\text { Minimum Peak in } \\
\text { Final Diff. Map / } \AA^{3}\end{array}$ & -0.41 \\
\hline Flack parameter & $-0.3(2)$ \\
\hline
\end{tabular}




\section{Computational Study}

\subsection{Calculation method details.}

All geometry optimizations and thermal energy correction calculations (frequency analyses) using density functional theory (DFT) were performed with the Gaussian 16 (revision C.01) ${ }^{15}$ suite of programs. The thresholds defined in Gaussian 16 were used. The geometry optimizations were carried out at $\omega \mathrm{B} 97 \mathrm{X}-\mathrm{D}^{16}$ level of theory with Def2-SVP ${ }^{17,18}$ basis set because this combination of the DFT model can reproduce experimental reactivities and selectivities of a various type of borylation reaction with copper(I)/diboron catalyst system. ${ }^{19-22}$ The solvation effect of DMF was included in the calculations using CPCM solvation model. ${ }^{23,24}$ Harmonic frequency calculations were conducted at the same level of theory on the optimized geometries to check all the stationary points as either minima or first-order saddle points. Intrinsic reaction coordinate (IRC) ${ }^{25}$ calculations were carried out to confirm the transition states connecting the correct reactants and products on the potential energy surface.

Then, the self-consistent field (SCF) energies of the optimized molecular systems were corrected at a higher level of theory. For this purpose, we chose $\omega \mathrm{B} 97 \mathrm{M}-\mathrm{V}^{26}$ functional with Def2-TZVPP 17,18 basis set because we need a method with high accuracy to compare two different types of the transition state (TS), coordination of borylcopper(I) species to allenes (TS1), and borylcupration of allenes (TS2). $\omega \mathrm{B} 97 \mathrm{M}-\mathrm{V}$ is one of the state-of-the-art DFT methods and showed excellent performance on many benchmarks involving non-covalent interactions, isomerization energies, thermochemical properties, barrier height, etc. of organic systems including transition metals. ${ }^{27-29}$ The single-point calculations were performed with the Orca 4.1.2 program. ${ }^{30,31}$ The solvation effect of DMF was included in the calculations using SMD solvation model. ${ }^{32}$ The calculated structures were visualized with VMD program. $^{33}$

Summary: $\omega$ B97M-V/Def2-TZVPP/SMD(DMF)// $\omega$ B97X-D/Def2-SVP/CPCM(DMF). 


\subsection{DFT study for the case of Xantphos ligand conditions.}

The DFT study on the regio- and stereoselectivity-determining steps in the case of the substrate 1b and Xantphos ligand was conducted (Figure S13), as well as the substrate 1a and SIMes ligand described in the main text (Figure 6).

In the major path to the allylic boronate $(E)-\mathbf{4 b y}$, the barrierless coordination of the boryl copper(I) species Int $\mathbf{2}$ to allene $\mathbf{1 b}$ forms the slightly unstable $\pi$-complex $\mathbf{E Q 2} \mathbf{2}^{\text {allyl-E}}$ relative to the precursor state EQ1 (EQ1: $\Delta G=0.0 \mathrm{kcal} / \mathrm{mol}, \mathbf{E Q 2}^{\text {allyl-E}}: \Delta G=7.3 \mathrm{kcal} / \mathrm{mol}$ ). The subsequent borylcupration generates the highly stable alkenyl copper(I) species Int3 $3^{\text {allyl- } E}$ via the small transition state TS2 $^{\text {allyl- } E}$ (TS2 $2^{\text {allyl-E}: ~} \Delta G^{\ddagger}=12.0 \mathrm{kcal} / \mathrm{mol}$, Int3 $3^{\text {allyl-E}}: \Delta G=-24.2 \mathrm{kcal} / \mathrm{mol}$ ). Thus, this borylcupration step is irreversible and the selectivity-determining step of this major path. For a path to the stereoisomer $(Z)$ 4by, the corresponding $\pi$-complex $\mathbf{E Q 2}^{\text {allyl- } Z}$ was not found on the IRC from the transition state of the coordination step TS1 ${ }^{\text {allyl-Z }}$ (TS1 ${ }^{\text {allyl-Z}}$ : $\Delta G^{\ddagger}=16.8 \mathrm{kcal} / \mathrm{mol}$ ). The IRC is directly connected to the alkenyl copper(I) species Int3 ${ }^{\text {allyl-Z }}$ (Int3 ${ }^{\text {allyl-Z}}$ : $\Delta G=-21.2 \mathrm{kcal} / \mathrm{mol}$ ). A path to the regioisomer (Z)5by, as well as the major path, has no TS1 corresponding to TS1 ${ }^{\text {alkenyl-Z }}$. Also, the borylcupration generates the alkenyl copper(I) Int3 $^{\text {alkenyl- } Z}$ via the transition state TS2 $^{\text {alkenyl- } Z}$ (TS2 ${ }^{\text {alkenyl- } Z}$ : $\Delta G^{\ddagger}=16.3$ $\mathrm{kcal} / \mathrm{mol}$, Int3 $\left.{ }^{\text {alkenyl-Z}: ~} \Delta G=-23.2 \mathrm{kcal} / \mathrm{mol}\right)$. Finally, a path to another regioisomer $(E)-5 \mathbf{b y}$ has an extremely high transition state of the borylcupration TS2 $^{\text {alkenyl-E}}$. However, the prior transition state of the coordination TS1 $^{\text {alkenyl- } E}$ is also higher than the selectivity-determining TS of the major path TS2 $^{\text {allyl-E }}$ (TS1 ${ }^{\text {alkenyl-E}}: \Delta G^{\ddagger}=13.8 \mathrm{kcal} / \mathrm{mol}$, TS2 $\left.^{\text {alkenyl- } E}: \Delta G^{\star}=31.7 \mathrm{kcal} / \mathrm{mol}\right)$. In summary, TS2 $^{\text {allyl-E}}$, TS1 $^{\text {allyl-Z}}$, TS2 ${ }^{\text {alkenyl-Z}}$, and TS2 ${ }^{\text {alkenyl- } E}$ are selectivity-determining TSs.

Structures of the respective selectivity-determining TSs were shown in Figure S13B. The predicted selectivities using relative Gibbs free energies are in good agreement with the experimental values (for 4ba, experimentally $E / Z=>95: 5, \mathbf{4 : 5}=>95: 5$; theoretically $E / Z=>99.9: 0.1,4: 5=$ $>99.9: 0.1)$. In the major TS TS2 $2^{\text {allyl-E}}$, the methyl (Me) group of allene $\mathbf{1 b}$ and the boryl [B(pin)] group incline into the pocket-like space of the catalyst. Therefore, the $\mathbf{T S 2}^{\text {allyl- } E}$ can be formed without large steric repulsions, although the 1,3-allylic strain is found in the substrate $\mathbf{1 b}\left[\Delta \Delta G^{\star}=0.00 \mathrm{kcal} / \mathrm{mol}\right.$, $\left.\Delta \Delta H^{\ddagger}=0.00 \mathrm{kcal} / \mathrm{mol}, \Delta\left(-T \Delta S^{\ddagger}\right)=0.00 \mathrm{kcal} / \mathrm{mol}\right]$. For the minor TS TS1 ${ }^{\text {allyl-Z}}$, the steric repulsion between the tert-butyl $(t-\mathrm{Bu})$ group and the catalyst causes a large enthalpic destabilization of the structure. However, this minor TS is slightly entropically favored than the major TS TS2 ${ }^{\text {allyl-E}}$ due to the absence of the 1,3-allylic repulsion $\left[\Delta \Delta G^{\star}=4.75 \mathrm{kcal} / \mathrm{mol}, \Delta \Delta H^{\star}=4.97 \mathrm{kcal} / \mathrm{mol}, \Delta\left(-T \Delta S^{\star}\right)=-\right.$ $0.22 \mathrm{kcal} / \mathrm{mol}]$. In the TS of one of the minor regioisomers, $\mathbf{T S 2}^{\text {alkenyl-Z }}$, the combination of the steric repulsion between the substrate and the $\mathrm{B}$ (pin) group, and the 1,3-allylic strain destabilizes the structure both enthalpically and entropically $\left[\Delta \Delta G^{\ddagger}=4.25 \mathrm{kcal} / \mathrm{mol}, \Delta \Delta H^{\ddagger}=2.41 \mathrm{kcal} / \mathrm{mol}, \Delta\left(-T \Delta S^{\ddagger}\right)\right.$ $=1.84 \mathrm{kcal} / \mathrm{mol}]$. Finally, for the TS of another regioisomer, TS2 $^{\text {alkenyl }-E}$, the tremendous steric repulsion between the $t$-Bu group and the $\mathrm{B}$ (pin) group induces the huge enthalpic destabilization of this structure $\left[\Delta \Delta G^{\ddagger}=19.72 \mathrm{kcal} / \mathrm{mol}, \Delta \Delta H^{\ddagger}=18.55 \mathrm{kcal} / \mathrm{mol}, \Delta\left(-T \Delta S^{\ddagger}\right)=1.18 \mathrm{kcal} / \mathrm{mol}\right]$. In 
conclusion, those minor paths are destabilized by predominantly enthalpic effect as described above, meanwhile, the desired isomer $(E)-\mathbf{4 b y}$ can be produced via the major TS TS2 ${ }^{\text {allyl- } E}$ without significant steric repulsion.

As shown in Figures 6B and S13A, the barrier of the selectivity-determining major TS for SIMes (Figure 6B, TS1 ${ }^{\text {allyl-E}}, \Delta G^{\dagger}=17.0 \mathrm{kcal} / \mathrm{mol}$ ) is larger than that for Xantphos (Figure S13A, TS2 $2^{\text {allyl-E}}$, $\Delta G^{\ddagger}=12.0 \mathrm{kcal} / \mathrm{mol}$ ). However, the reaction with SIMes ligand proceeds under a lower catalyst loading and reaction temperature than that with Xantphos (Table 1). We speculate that this conflict between the reaction barrier and the reactivity of those ligands is because the alkylation step (Int4 to Int5) rather than the coordination TS1 and borylcupration TS2 is the turnover-limiting step (Figure 6A). The alkylation with Xantphos ligand should be disfavored compared to SIMes ligand as the bidentate of Xantphos to the copper(I) reaction center sterically and electronically suppress the additional coordination of alkoxide base to make the following alkylation disadvantageous. Thus, Xantphos ligand showed lower reactivity than SIMes ligand.
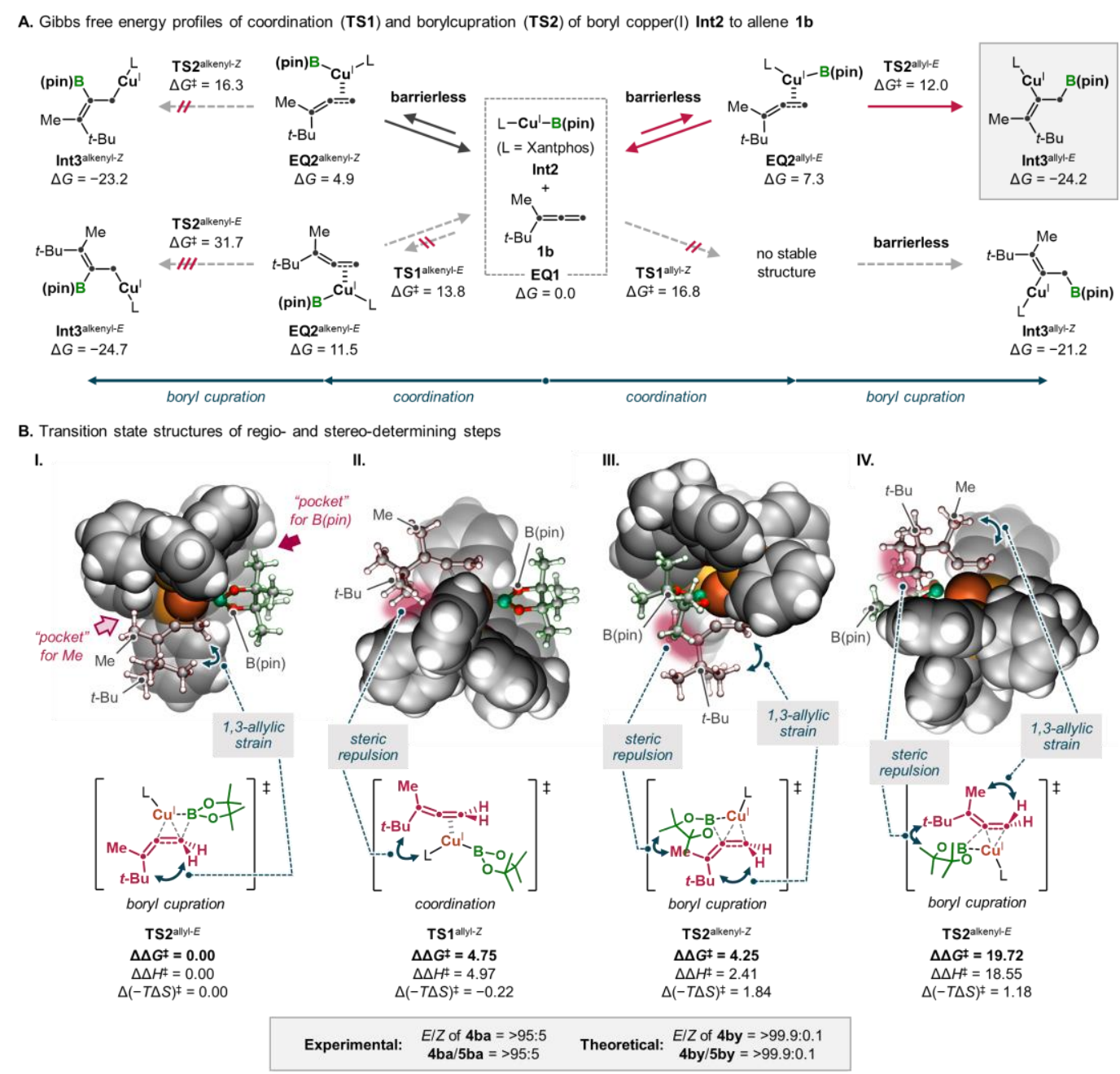

Figure S13. Gibbs free energy profiles and transition state structure with Xantphos ligand; energy values are given in $\mathrm{kcal} / \mathrm{mol}$. 


\subsection{Discussion about ligand effect.}

As discussed in Tables 1 and 2, SIMes is the optimal ligand for the allene substrates bearing a small $\mathrm{R}^{1}$ and a medium $\mathrm{R}^{2}$ substituent in this alkylboration reaction. In contrast, Xantphos ligand is optimal for the substrate bearing a small $\mathrm{R}^{1}$ and a large $\mathrm{R}^{2}$ substituent. However, the product was obtained with moderate stereoselectivity when Xantphos ligand was applied to the substrates bearing a small $\mathrm{R}^{1}$ and a medium $\mathrm{R}^{2}$ substituent (e.g., 1a; Table 1 , entry $1, E / Z$ of $4 \mathbf{a a}=60: 40$ ). Also, the combination of SIMes and the substrate bearing a small $\mathrm{R}^{1}$ and a large $\mathrm{R}^{2}$ resulted in the isomer mixture (e.g., 1b; Table 1, entry 13, 77:23 mixture of isomers, unfortunately, the structure of the minor isomer could not be identified).

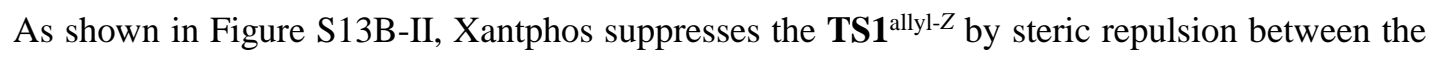
ligand and the $\mathrm{R}^{2}$ substituent. Thus, the smaller $\mathrm{R}^{2}$ substituent is, the more stable the TS1 ${ }^{\text {allyl- } E}$ becomes. Although the smaller $\mathrm{R}^{2}$ substituent should also make the 1,3-allylic strain in the $\mathbf{T S 2}^{2}{ }^{\text {ally- } E}$ smaller, the degree of stabilization of TS1 ${ }^{\text {allyl- } Z}$ would be larger than that of $\mathbf{T S 2}^{\text {allyl-Z }}$. Consequently, the stereoselectivity of the substrate bearing a medium $\mathrm{R}^{2}$ is decreased in the reaction with Xantphos ligand.

On the other hand, we then focus on combining SIMes ligand and the substrate bearing a large $\mathrm{R}^{2}$. As shown in Figures $6 \mathrm{~B}$ and $6 \mathrm{C}-\mathrm{I}$, TS1 ${ }^{\text {allyl- } E}$ is the selectivity-determining step because TS1 ${ }^{\text {allyl- } E}$ is larger than TS2 ${ }^{\text {allyl-E}}$ in the case of SIMes ligand and the substrate 1a. However, we speculate that replacing the Cy group in 1a with larger substituent cause destabilization of TS2 $2^{\text {allyl-E }}$ to make this TS higher than TS1 $^{\text {allyl-E}}$ because the increasing 1,3-allylic strain along the borylcupration via TS2 ${ }^{\text {allyl- } E}$ become significant. Also, this large destabilization of $\mathbf{T S 2}^{2}{ }^{\text {allyl-E}}$ makes this major path more disfavored to cause the decrease of the selectivity. 


\subsection{Relationship between ligand structure and reaction selectivities.}

To analyze the relationship between the ligand structure and the regio- and stereoselectivities of borylcupration of allenes, the steric environment of the ligand was investigated by the steric map and a newly defined "octant model" instead of "quadrant model" (Figure S14). The "quadrant model" is a frequently used intuitive tool describing ligand structures, especially the asymmetric environment of chiral ligands. Here we defined the "octant model" to discuss the ligand structure more precisely (Figure S14-I). All directions were divided into eight regions as the copper(I) atom is the center, from NNE to NNW clockwisely. Each region is highlighted with a five-step grayscale according to the degree of the steric hindrance concerning the steric map. For example, in the case of Xantphos/CuCl, there are two hindered regions around the east (E) and west (W), and two non-hindered regions around the north (N) and south (S) (Figure S14-II).
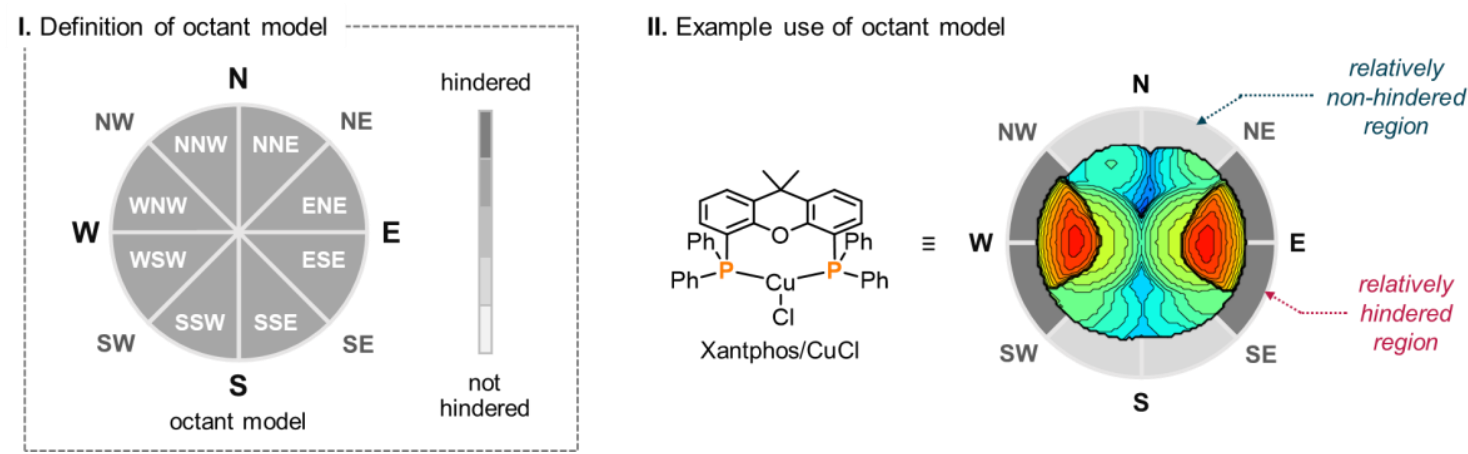

Figure S14. Qualitative visualization of the steric environment of ligands.

The experimental selectivities, octant models, and steric maps of the ligands investigated in Table 1 are shown in Figure S15. SIMes, IMes, IAd, and Xantphos showed excellent regioselectivities and have almost the same patterns of the steric environment, which has two pocket-like structures at the north $(\mathrm{N})$ and south $(\mathrm{S})$ regions $(\mathbf{4} / \mathbf{5}=93: 7->99: 1)$. Contrary, dppp bearing steric hindrance at the $\mathrm{N}$ region showed decreased regioselectivity $(\mathbf{4} / \mathbf{5}=44: 56)$. Furthermore, dppf bearing two steric hindrances at the NNW and SSE regions showed inverted regioselectivity $(4 / 5=6: 94)$. Therefore, we assumed that the presence of the two pockets for the boryl group and the small substituent $\mathrm{R}^{1}$ around the $\mathrm{N}$ and $\mathrm{S}$ regions is crucial to high regioselectivities of borylcupration and the resulting borylation product.

We then focus on the relationship between the ligand structure and the stereoselectivity. SIMes and IMes showed excellent stereoselectivity for substrate 1a, while the selectivity was dropped with IAd and Xantphos. As discussed in Figure 7 in the main text, the steric repulsion between the ligand and the $\mathrm{R}^{2}$ substituent in $\mathbf{T S 1} 1^{\text {allyl- } Z}$ is necessary for high stereoselectivity. Thus, we speculate that a too 
deep pocket for the $\mathrm{R}^{2}$ substituent would decrease the stereoselectivity. The pockets of IAd and Xantphos would be too deep to distinguish the small $\mathrm{R}^{1}$ substituent and medium $\mathrm{R}^{2}$ substituent.

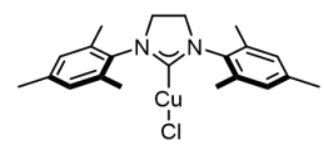

SIMesCuCl

$E / Z=>95: 5$ $\mathbf{4} / \mathbf{5}=93: 7$

III.

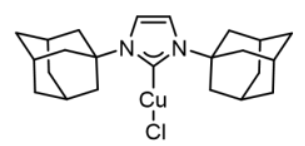

IAdCuCl

$E / Z=32: 68$

$4 / 5=98: 2$

V.<smiles>ClC([PH](Cl)(c1ccccc1)c1ccccc1)[PH](c1ccccc1)(c1ccccc1)c1ccccc1</smiles>

dppp/CuCl

$E / Z=70: 30$ $\mathbf{4} / \mathbf{5}=44: 56$ w

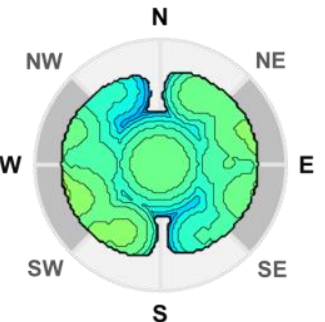

N

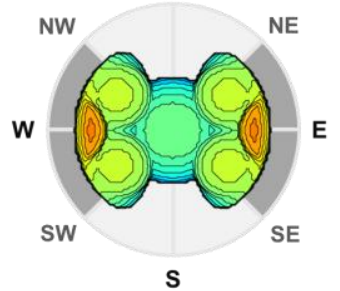

N

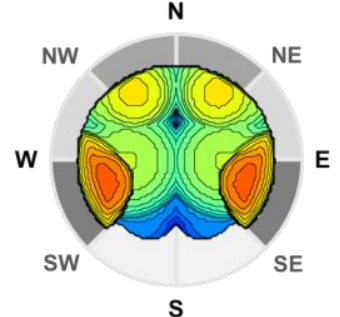

II.<smiles></smiles>

IMesCuCl

$E / Z=>95: 5$

$\mathbf{4} / \mathbf{5}=94: 6$

IV.

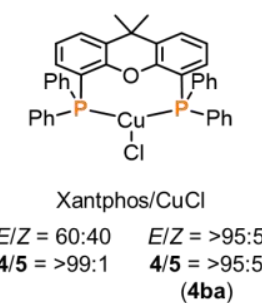

(4ba)

VI.

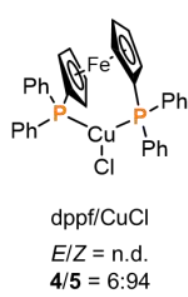

N

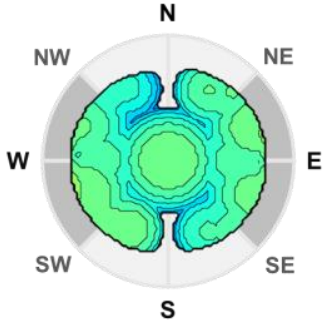

N
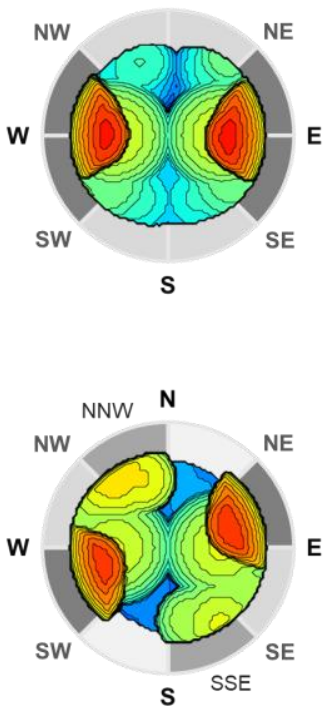

Figure S15. Experimental selectivities of the carboboration of 4aa and octant model of respective ligands. 


\subsection{Calculated properties of all structures.}

Table S5. Calculated energies and thermochemical parameters of the optimized structures.

\begin{tabular}{|c|c|c|c|c|}
\hline Structure & $\begin{array}{c}E \\
{[\text { hartree] }}\end{array}$ & $\begin{array}{c}H \\
{[\text { hartree] }}\end{array}$ & $\begin{array}{c}T S \\
{[\text { hartree] }}\end{array}$ & $\begin{array}{c}G \\
{[\text { hartree] }}\end{array}$ \\
\hline \multicolumn{5}{|c|}{ Ligand: SIMes, substrate: $1 \mathrm{a}$} \\
\hline Catalyst Int2 & -2897.899856 & -2897.898912 & -0.102980 & -2898.001892 \\
\hline Substrate 1a & -390.355657 & -390.354713 & -0.048561 & -390.403274 \\
\hline TS1 ${ }^{\text {allyl- } E}$ & -3288.257399 & -3288.256454 & -0.121639 & -3288.378093 \\
\hline TS1 $^{\text {allyl-Z }}$ & -3288.254904 & -3288.253960 & -0.121093 & -3288.375053 \\
\hline TS1 $^{\text {alkenyl- } E}$ & -3288.256106 & -3288.255162 & -0.124582 & -3288.379744 \\
\hline TS1 $^{\text {alkenyl- } Z}$ & -3288.260313 & -3288.259369 & -0.122300 & -3288.381669 \\
\hline $\mathbf{E Q 2}^{\text {allyl- } E}$ & -3288.261781 & -3288.260837 & -0.122604 & -3288.383441 \\
\hline $\mathbf{E Q 2}^{\text {allyl-Z }}$ & -3288.261602 & -3288.260658 & -0.121819 & -3288.382477 \\
\hline $\mathbf{E Q 2}^{\text {alkenyl- } E}$ & -3288.266323 & -3288.265379 & -0.122734 & -3288.388113 \\
\hline $\mathbf{E Q 2}^{\text {alkenyl-Z }}$ & -3288.267039 & -3288.266095 & -0.121835 & -3288.387930 \\
\hline TS2 $^{\text {allyl- } E}$ & -3288.256618 & -3288.255674 & -0.123364 & -3288.379038 \\
\hline TS2 ${ }^{\text {allyl-Z }}$ & -3288.259006 & -3288.258062 & -0.120780 & -3288.378842 \\
\hline TS2 $^{\text {alkenyl- } E}$ & -3288.253543 & -3288.252598 & -0.120554 & -3288.373152 \\
\hline TS2 $^{\text {alkenyl- } Z}$ & -3288.254710 & -3288.253766 & -0.120446 & -3288.374212 \\
\hline Int3 ${ }^{\text {allyl- } E}$ & -3288.322829 & -3288.321885 & -0.121583 & -3288.443468 \\
\hline Int3 $^{\text {allyl-Z }}$ & -3288.325142 & -3288.324198 & -0.123632 & -3288.447830 \\
\hline Int3 $3^{\text {alkenyl-E }}$ & -3288.327429 & -3288.326485 & -0.119278 & -3288.445763 \\
\hline Int3 $3^{\text {alkenyl- } Z}$ & -3288.328378 & -3288.327434 & -0.119909 & -3288.447343 \\
\hline \multicolumn{5}{|c|}{ Ligand: Xantphos, substrate: $1 \mathrm{~b}$} \\
\hline Catalyst Int2 & -4314.300915 & -4314.299970 & -0.132457 & -4314.432427 \\
\hline Substrate $\mathbf{1 b}$ & -312.971634 & -312.970690 & -0.045390 & -313.016080 \\
\hline TS1 $^{\text {allyl- } Z}$ & -4627.272228 & -4627.271284 & -0.150487 & -4627.421771 \\
\hline TS1 $^{\text {alkenyl- } Z}$ & -4627.277299 & -4627.276354 & -0.150181 & -4627.426535 \\
\hline $\mathbf{E Q 2}^{\text {allyl- } E}$ & -4627.284956 & -4627.284012 & -0.152872 & -4627.436884 \\
\hline $\mathbf{E Q 2}^{\text {alkenyl- } E}$ & -4627.290122 & -4627.289177 & -0.151570 & -4627.440747 \\
\hline $\mathbf{E Q 2}^{\text {alkenyl- } Z}$ & -4627.281502 & -4627.280557 & -0.149602 & -4627.430159 \\
\hline $\mathbf{T S 2}^{\text {allyl-E}}$ & -4627.280154 & -4627.279210 & -0.150130 & -4627.429340 \\
\hline TS2 $^{\text {alkenyl- } E}$ & -4627.276318 & -4627.275374 & -0.147191 & -4627.422565 \\
\hline TS2 $^{\text {alkenyl- } Z}$ & -4627.250600 & -4627.249656 & -0.148257 & -4627.397913 \\
\hline Int3 ${ }^{\text {allyl-E }}$ & -4627.337341 & -4627.336397 & -0.149024 & -4627.485421 \\
\hline Int3 ${ }^{\text {allyl-Z }}$ & -4627.339475 & -4627.338530 & -0.149307 & -4627.487837 \\
\hline Int3 $3^{\text {alkenyl-E }}$ & -4627.337375 & -4627.336430 & -0.150686 & -4627.487116 \\
\hline Int3 $3^{\text {alkenyl- } Z}$ & -4627.332616 & -4627.331671 & -0.150579 & -4627.482250 \\
\hline
\end{tabular}




\section{References}

1. Sheldrick, G. M. SHELXL-2013, Program for the Refinement of Crystal Structures; University of Göttingen, Göttingen, Germany, 2013.

2. Liu, J.; Nie, M.; Zhou, Q.; Gao, S.; Jiang, W.; Chung, L. W.; Tang, W.; Ding, K. Enantioselective Palladium-Catalyzed Diboration of 1,1-Disubstituted Allenes. Chem. Sci. 2017, 8, 5161-5165.

3. Pasto, D. J.; Warren, S. E.; Morrison, M. A. Radical-Chain Addition of Benzenethiol to Alienes. Analysis of Steric Effects and Reversibility. J. Org. Chem. 1981, 46, 2837-2841.

4. Köpfer, A.; Breit, B. Rhodium-Catalyzed Hydroformylation of 1,1-Disubstituted Allenes Employing the Self-Assembling 6-DPPon System. Angew. Chem., Int. Ed. 2015, 54, 6913-6917.

5. Takaya, J.; Iwasawa, N. Hydrocarboxylation of Allenes with CO2 Catalyzed by Silyl PincerType Palladium Complex. J. Am. Chem. Soc. 2008, 130, 15254-15255.

6. Wang, B.; Gandamana, D. A.; Gagosz, F.; Chiba, S. Diastereoselective Intramolecular Hydride Transfer under Brønsted Acid Catalysis. Org. Lett. 2019, 21, 2298-2301.

7. Wang, C.; Teo, W. J.; Ge, S. Access to Stereodefined (Z)-Allylsilanes and (Z)-Allylic Alcohols via Cobalt-Catalyzed Regioselective Hydrosilylation of Allenes. Nat. Commun. 2017, 8, 1-9.

8. Liu, X.; Liu, B.; Liu, Q. Migratory Hydrogenation of Terminal Alkynes by Base/Cobalt Relay Catalysis. Angew. Chem., Int. Ed. 2020, 59, 6750-6755.

9. Santoro, O.; Collado, A.; Slawin, A. M. Z.; Nolan, S. P.; Cazin, C. S. J. A General Synthetic Route to $[\mathrm{Cu}(\mathrm{X})(\mathrm{NHC})](\mathrm{NHC}=\mathrm{N}$-Heterocyclic Carbene, $\mathrm{X}=\mathrm{Cl}, \mathrm{Br}$, I) Complexes. Chem. Commun. 2013, 49, 10483-10485.

10. Mlynarski, S. N.; Karns, A. S.; Morken, J. P. Direct Stereospecific Amination of Alkyl and Aryl Pinacol Boronates. J. Am. Chem. Soc. 2012, 134, 16449-16451.

11. Sadhu, K. M.; Matteson, D. S. (Chloromethyl)Llthium: Efficient Generation and Capture by Boronic Esters and a Simple Preparation of Diisopropyl (Chloromethyl)Boronate. Organometallics 1985, 4, 1687-1689.

12. Zanghi, J. M.; Liu, S.; Meek, S. J. Enantio- and Diastereoselective Synthesis of Functionalized Carbocycles by Cu-Catalyzed Borylative Cyclization of Alkynes with Ketones. Org. Lett. 2019, $21,5172-5177$.

13. Ishiyama, T.; Ahiko, T. A.; Miyaura, N. Acceleration Effect of Lewis Acid in Allylboration of Aldehydes: Catalytic, Regiospecific, Diastereospecific, and Enantioselective Synthesis of Homoallyl Alcohols. J. Am. Chem. Soc. 2002, 124, 12414-12415.

14. Otte, D. A. L.; Borchmann, D. E.; Lin, C.; Weck, M.; Woerpel, K. A. ${ }^{13}$ C NMR Spectroscopy for the Quantitative Determination of Compound Ratios and Polymer End Groups. Org. Lett. 2014, 16, 1566-1569. 
15. Gaussian 16, Revision C.01, M. J. Frisch, G. W. Trucks, H. B. Schlegel, G. E. Scuseria, M. A. Robb, J. R. Cheeseman, G. Scalmani, V. Barone, G. A. Petersson, H. Nakatsuji, X. Li, M. Caricato, A. V. Marenich, J. Bloino, B. G. Janesko, R. Gomperts, B. Mennucci, H. P. Hratchian, J. V. Ortiz, A. F. Izmaylov, J. L. Sonnenberg, D. Williams-Young, F. Ding, F. Lipparini, F. Egidi, J. Goings, B. Peng, A. Petrone, T. Henderson, D. Ranasinghe, V. G. Zakrzewski, J. Gao, N. Rega, G. Zheng, W. Liang, M. Hada, M. Ehara, K. Toyota, R. Fukuda, J. Hasegawa, M. Ishida, T. Nakajima, Y. Honda, O. Kitao, H. Nakai, T. Vreven, K. Throssell, J. A. Montgomery, Jr., J. E. Peralta, F. Ogliaro, M. J. Bearpark, J. J. Heyd, E. N. Brothers, K. N. Kudin, V. N. Staroverov, T. A. Keith, R. Kobayashi, J. Normand, K. Raghavachari, A. P. Rendell, J. C. Burant, S. S. Iyengar, J. Tomasi, M. Cossi, J. M. Millam, M. Klene, C. Adamo, R. Cammi, J. W. Ochterski, R. L. Martin, K. Morokuma, O. Farkas, J. B. Foresman, and D. J. Fox, Gaussian, Inc., Wallingford CT, 2016.

16. Chai, J. Da; Head-Gordon, M. Long-Range Corrected Hybrid Density Functionals with Damped Atom-Atom Dispersion Corrections. Phys. Chem. Chem. Phys. 2008, 10, 6615-6620.

17. Weigend, F.; Ahlrichs, R. Balanced Basis Sets of Split Valence, Triple Zeta Valence and Quadruple Zeta Valence Quality for H to Rn: Design and Assessment of Accuracy Phys. Chem. Chem. Phys. 2005, 7, 3297-3305.

18. Weigend, F. Accurate Coulomb-fitting Basis Sets for H to Rn, Phys. Chem. Chem. Phys. 2006, 8, $1057-1065$.

19. Meng, F.; Li, X.; Torker, S.; Shi, Y.; Shen, X.; Hoveyda, A. H. Catalytic Enantioselective 1,6Conjugate Additions of Propargyl and Allyl Groups. Nature 2016, 537, 387-393.

20. Lee, J.; Radomkit, S.; Torker, S.; Pozo, J.; Hoveyda, A. H. Mechanism-Based Enhancement of Scope and Enantioselectivity for Reactions Involving a Copper-Substituted Stereogenic Carbon Centre. Nat. Chem. 2018, 10, 99-108.

21. Ozawa, Y.; Iwamoto, H.; Ito, H. Copper(I)-Catalysed Regio- and Diastereoselective Intramolecular Alkylboration of Terminal Allenes: Via Allylcopper(I) Isomerization. Chem. Commun. 2018, 54, 4991-4994.

22. Iwamoto, H.; Ozawa, Y.; Takenouchi, Y.; Imamoto, T.; Ito, H. Backbone-Modified $C_{2}-$ Symmetrical Chiral Bisphosphine TMS-QuinoxP*: Asymmetric Borylation of Racemic Allyl Electrophiles. J. Am. Chem. Soc. 2021, 143, 6413-6422.

23. Barone, V.; Cossi, M. Quantum Calculation of Molecular Energies and Energy Gradients in Solution by a Conductor Solvent Model. J. Phys. Chem. A 1998, 102, 1995-2001.

24. Cossi, M.; Rega, N.; Scalmani, G.; Barone, V. Energies, Structures, and Electronic Properties of Molecules in Solution with the C-PCM Solvation Model. J. Comput. Chem. 2003, 24, 669-681.

25. Fukui, K. The Path of Chemical Reactions - The IRC Approach, Acc. Chem. Res. 1981, 14, 363368. 
26. Mardirossian, N.; Head-Gordon, M. $\omega \mathrm{B} 97 \mathrm{M}-\mathrm{V}$ : A Combinatorially Optimized, Range-Separated Hybrid, Meta-GGA Density Functional with VV10 Nonlocal Correlation. J. Chem. Phys. 2016, 144.

27. Mardirossian, N.; Head-Gordon, M. Thirty Years of Density Functional Theory in Computational Chemistry: An Overview and Extensive Assessment of 200 Density Functionals. Mol. Phys. 2017, $115,2315-2372$.

28. Najibi, A.; Goerigk, L. The Nonlocal Kernel in van Der Waals Density Functionals as an Additive Correction: An Extensive Analysis with Special Emphasis on the B97M-V and $\omega$ B97M-V Approaches. J. Chem. Theory Comput. 2018, 14, 5725-5738.

29. Vogiatzis, K. D.; Polynski, M. V.; Kirkland, J. K.; Townsend, J.; Hashemi, A.; Liu, C.; Pidko, E. A. Computational Approach to Molecular Catalysis by 3d Transition Metals: Challenges and Opportunities. Chem. Rev. 2019, 119, 2453-2523.

30. Neese, F. The ORCA Program System. Wiley Interdiscip. Rev.: Comput. Mol. Sci. 2012, 2, 73.

31. Neese, F. Software Update: the ORCA Program System, Version 4.0. Wiley Interdiscip. Rev.: Comput. Mol. Sci. 2017, 8, e1327.

32. Marenich, A. V.; Cramer, C. J.; Truhlar, D. G. Universal Solvation Model Based on Solute Electron Density and on a Continuum Model of the Solvent Defined by the Bulk Dielectric Constant and Atomic Surface Tensions, J. Phys. Chem. B 2009, 113, 6378-6396.

33. Humphrey, W.; Dalke, A.; Schulten, K. VMD: Visual Molecular Dynamics, J. Mol. Graph. 1996, 14, 33-38.

34. Boreux, A.; Indukuri, K.; Gagosz, F.; Riant, O. Acyl Fluorides as Efficient Electrophiles for the Copper-Catalyzed Boroacylation of Allenes. ACS Catal. 2017, 7, 8200-8204.

35. Xiong, M.; Xie, X.; Liu, Y. Copper-Catalyzed Borylative Cyclization of in Situ Generated oAllenylaryl Nitriles with Bis(pinacolato)diboron. Org. Lett. 2017, 19, 3398-3401.

36. Rae, J.; Yeung, K.; McDouall, J. J. W.; Procter, D. J. Copper-Catalyzed Borylative CrossCoupling of Allenes and Imines: Selective Three-Component Assembly of Branched Homoallyl Amines. Angew. Chem., Int. Ed. 2016, 55, 1102-1107.

37. Yeung, K.; Ruscoe, R. E.; Rae, J.; Pulis, A. P.; Procter, D. J. Enantioselective Generation of Adjacent Stereocenters in a Copper-Catalyzed Three-Component Coupling of Imines, Allenes, and Diboranes. Angew. Chem., Int. Ed. 2016, 55, 11912-11916.

38. Fujihara, T.; Sawada, A.; Yamaguchi, T.; Tani, Y.; Terao, J.; Tsuji, Y. Boraformylation and Silaformylation of Allenes. Angew. Chem., Int. Ed. 2017, 56, 1539-1543.

39. Sawada, A.; Fujihara, T.; Tsuji, Y. Copper-Catalyzed Bora-Acylation and BoraAlkoxyoxalylation of Allenes. Adv. Synth. Catal. 2018, 360, 2621-2625. 


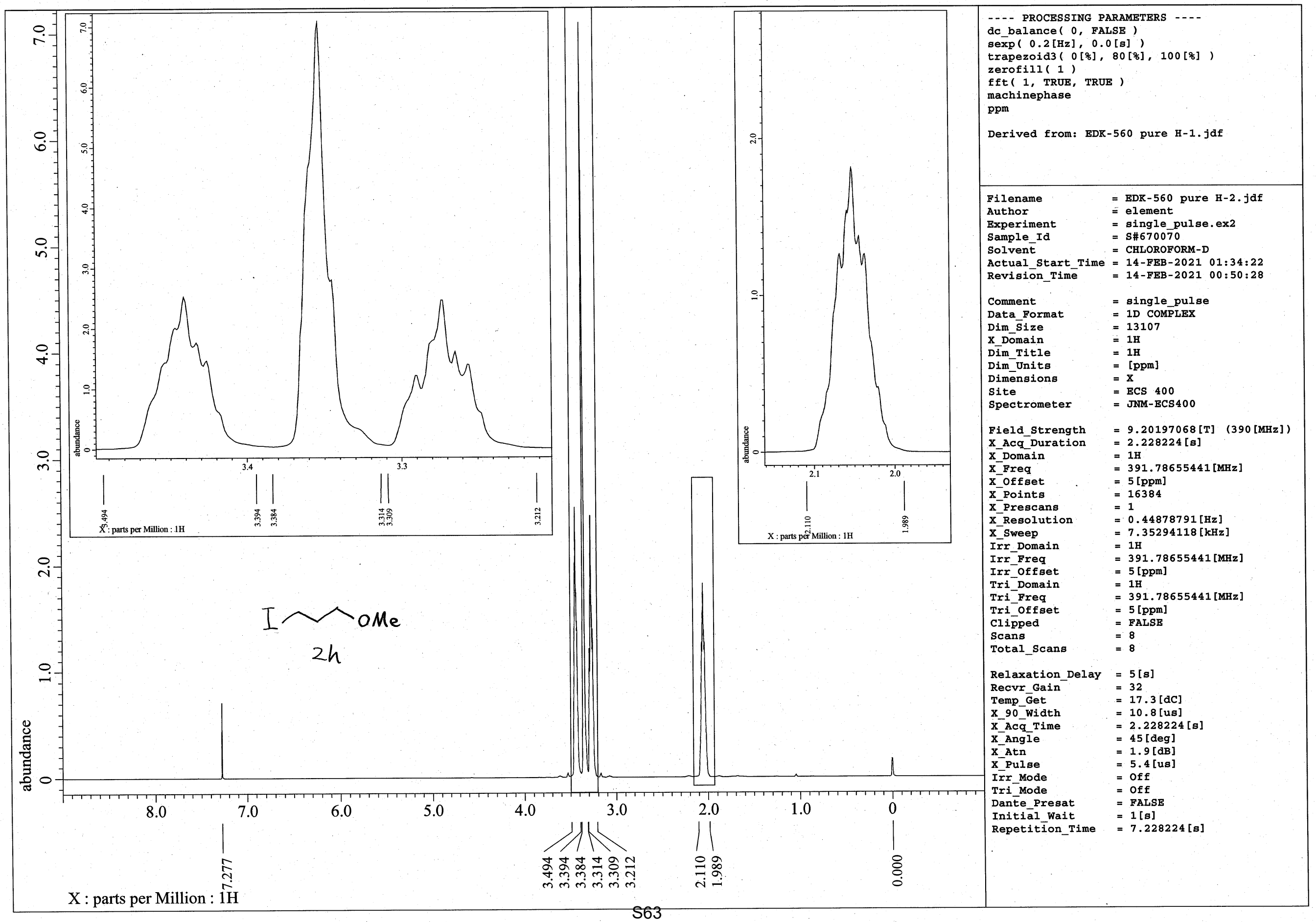




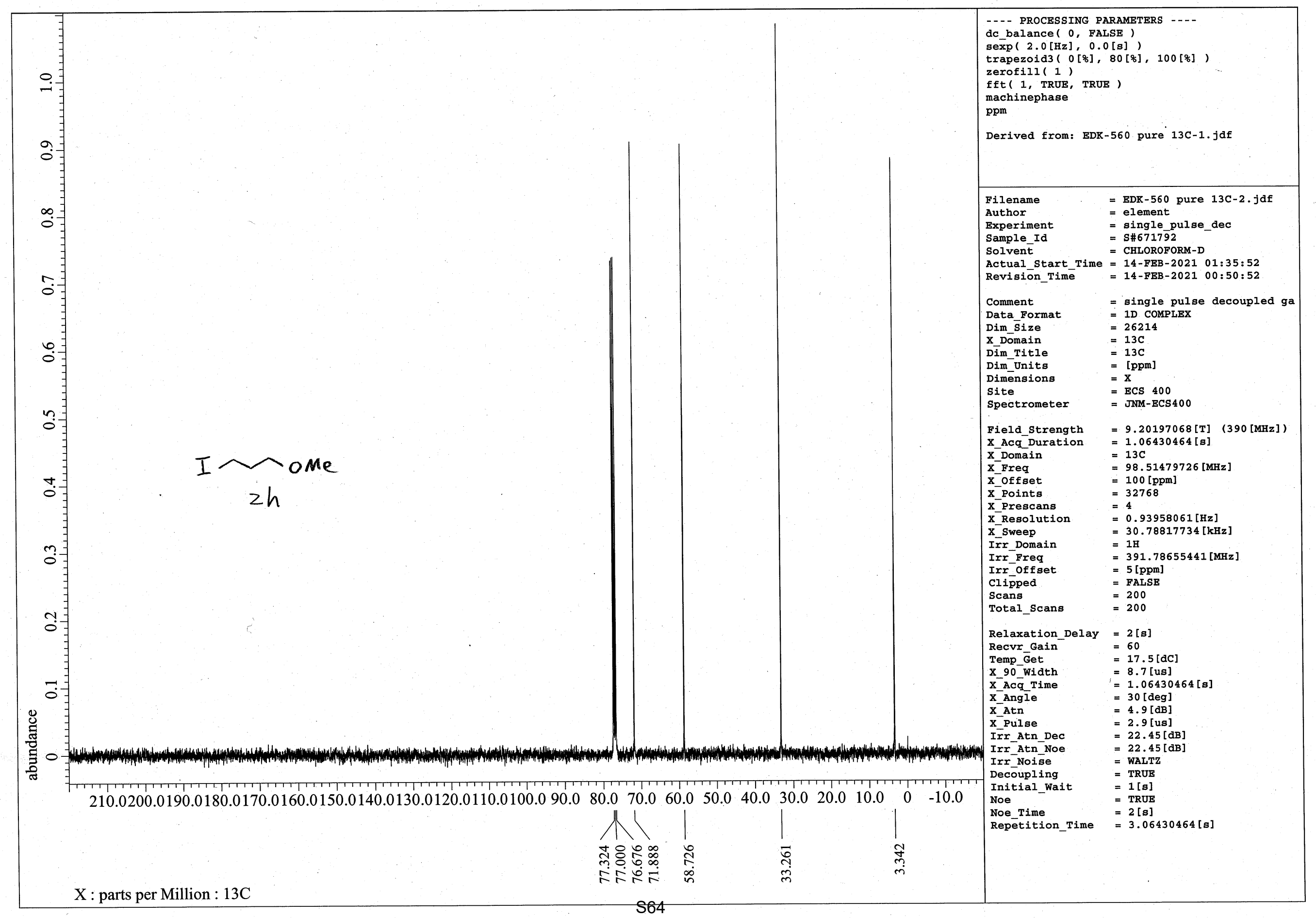




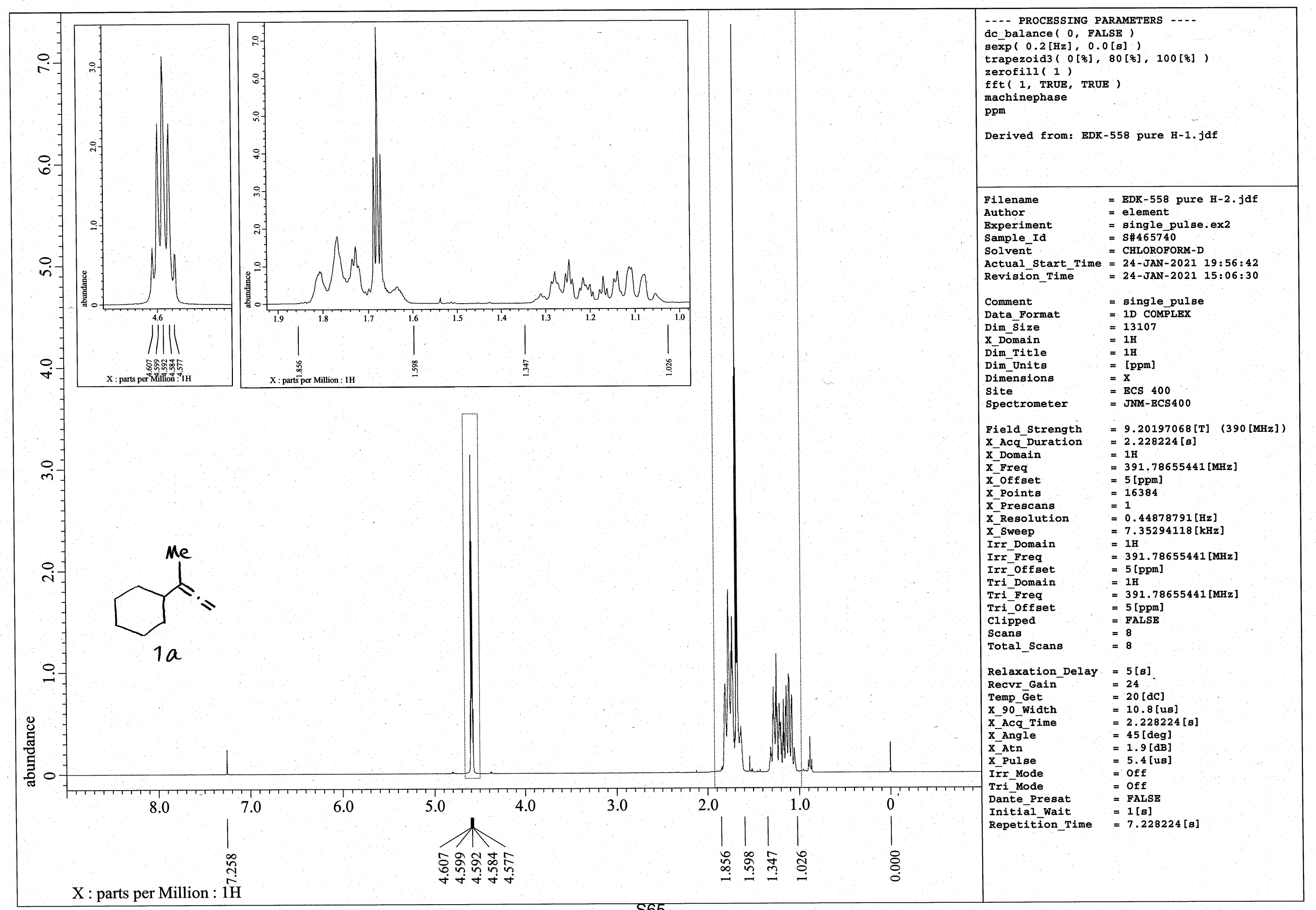




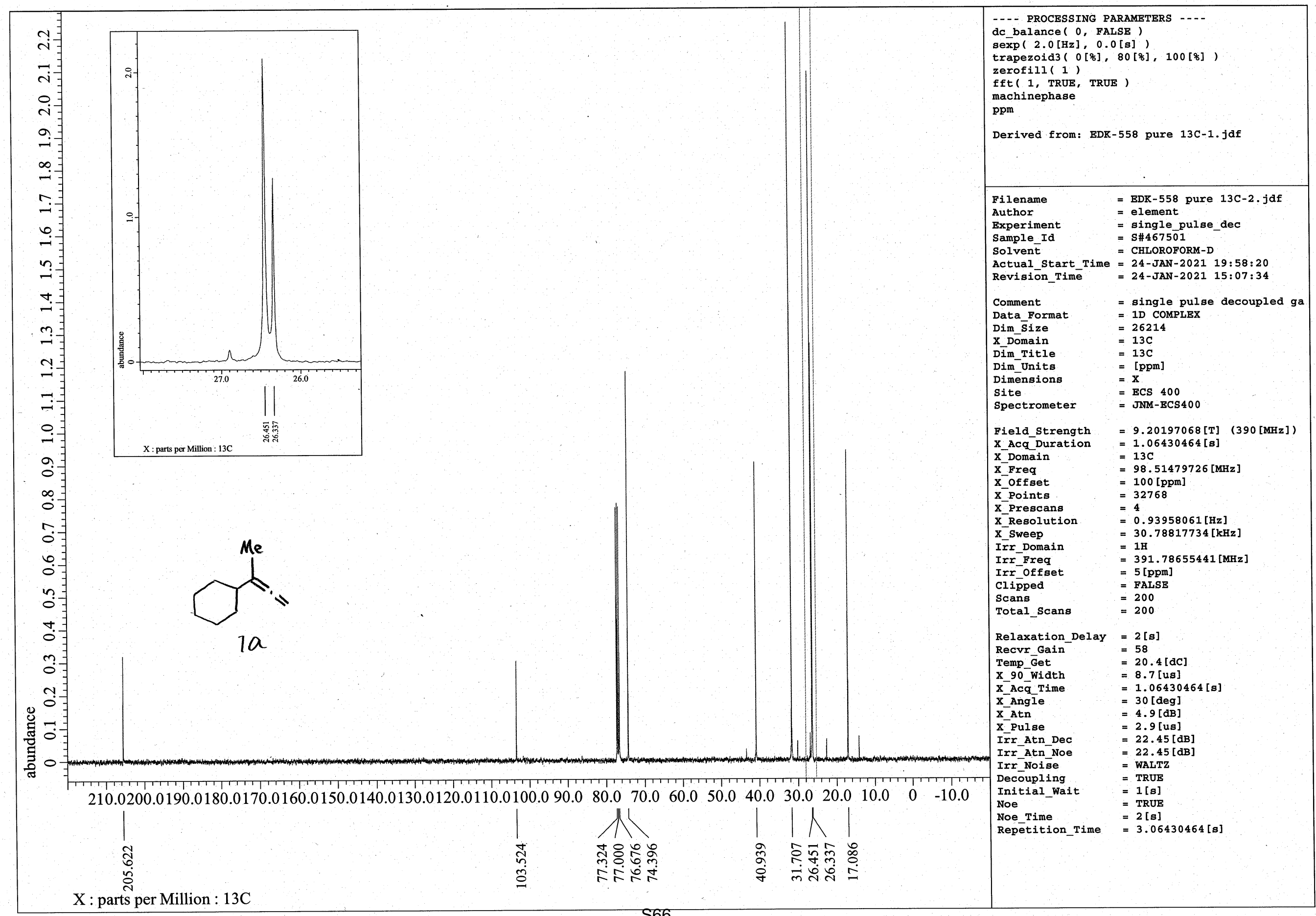




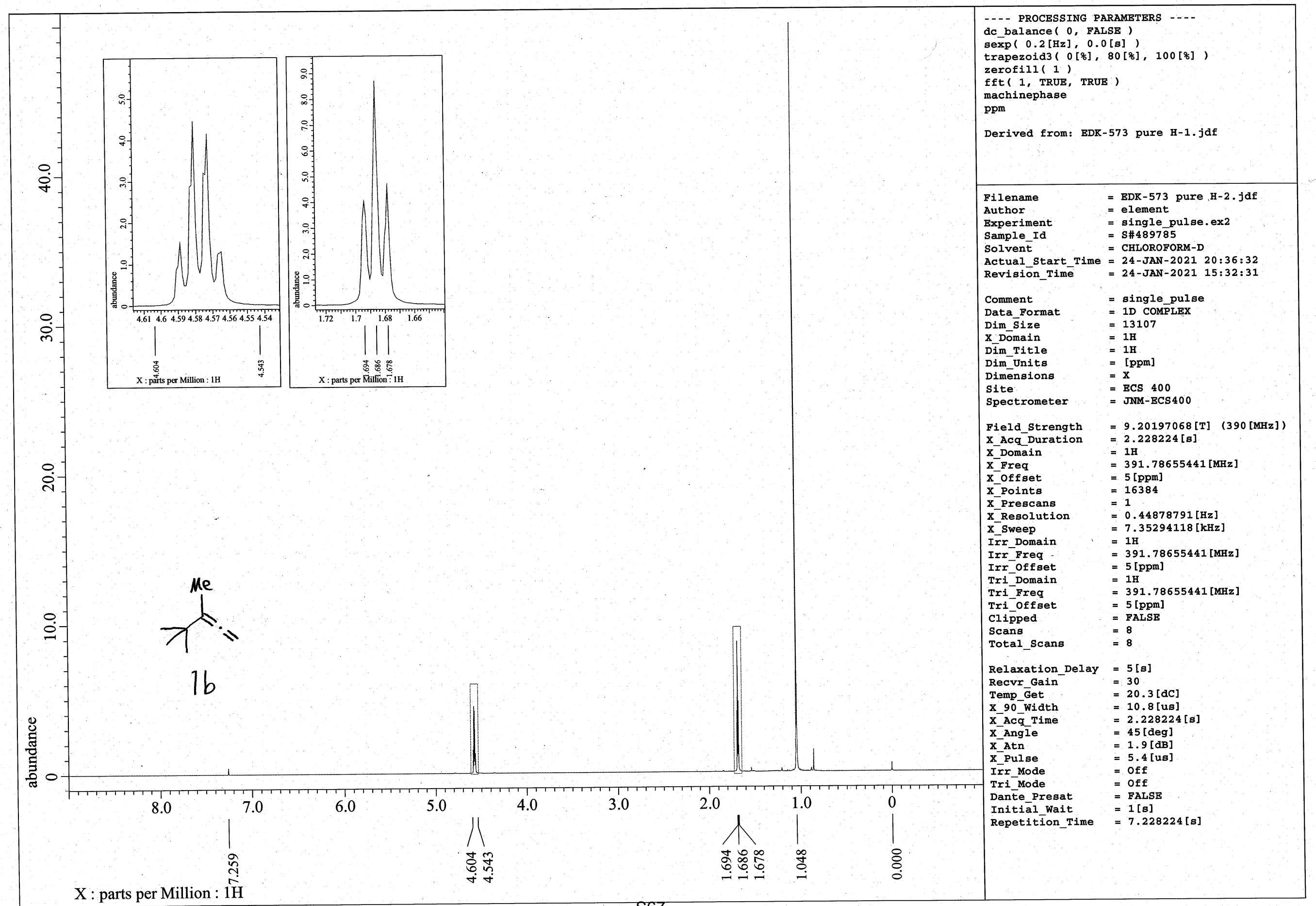




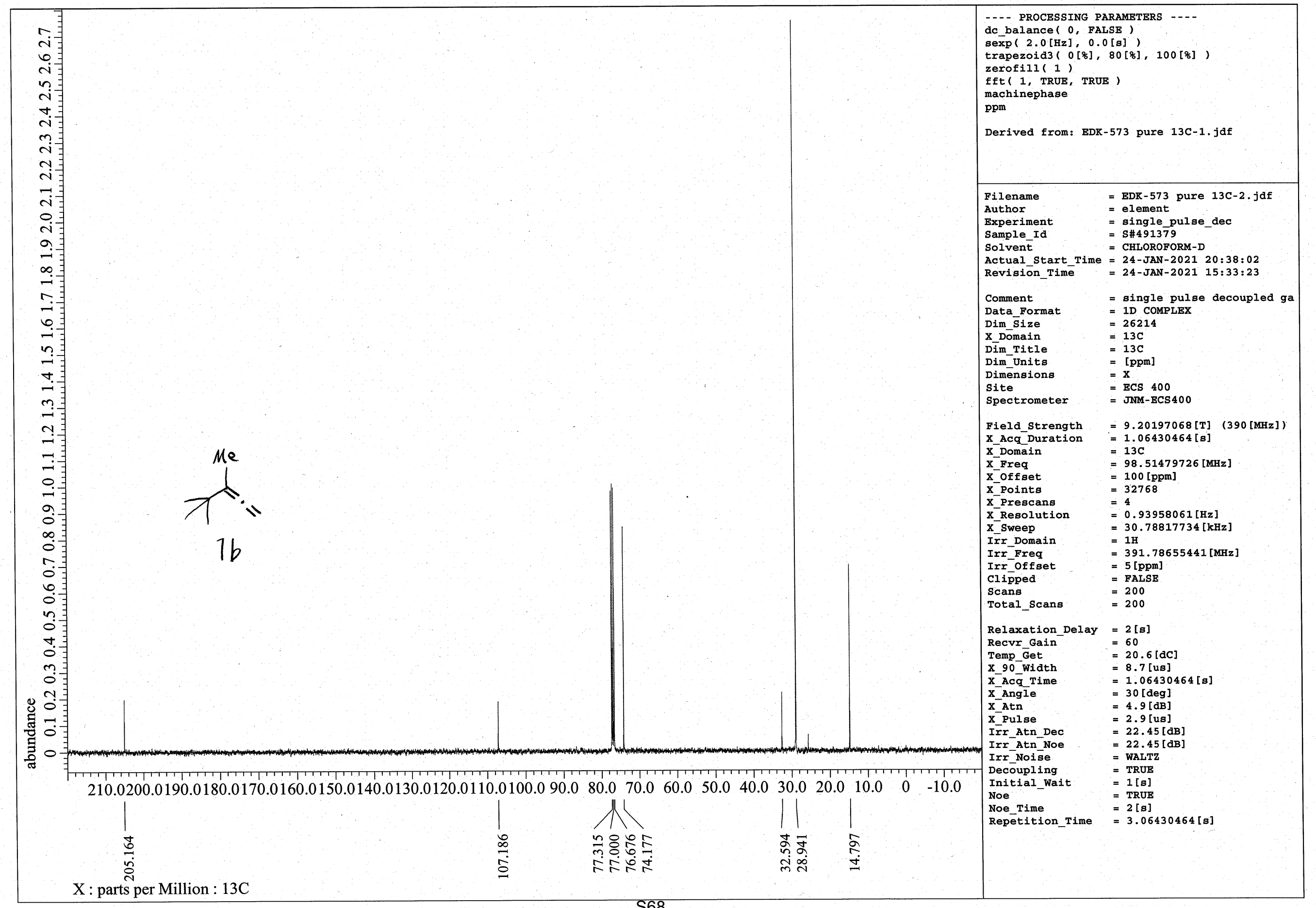




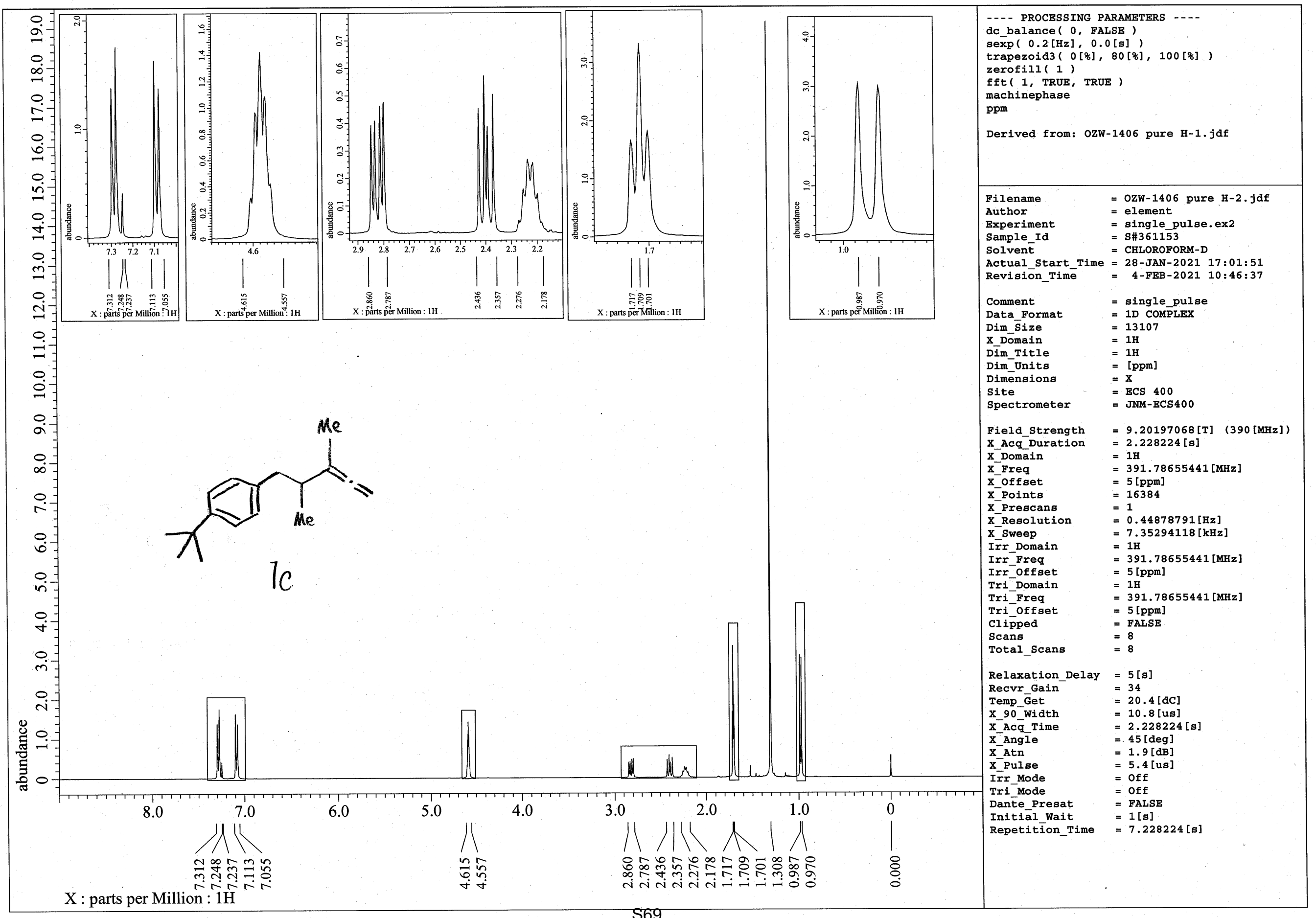




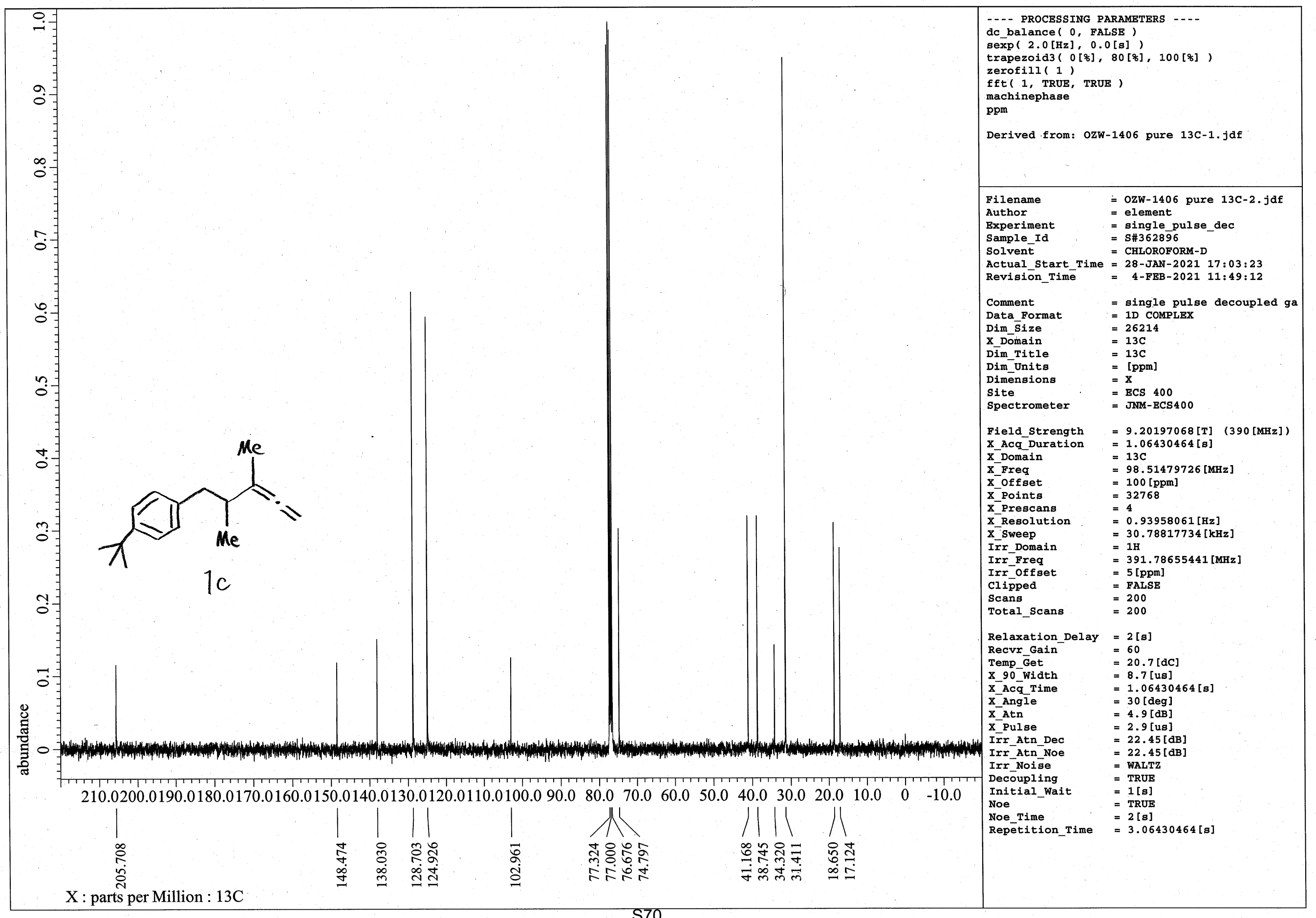




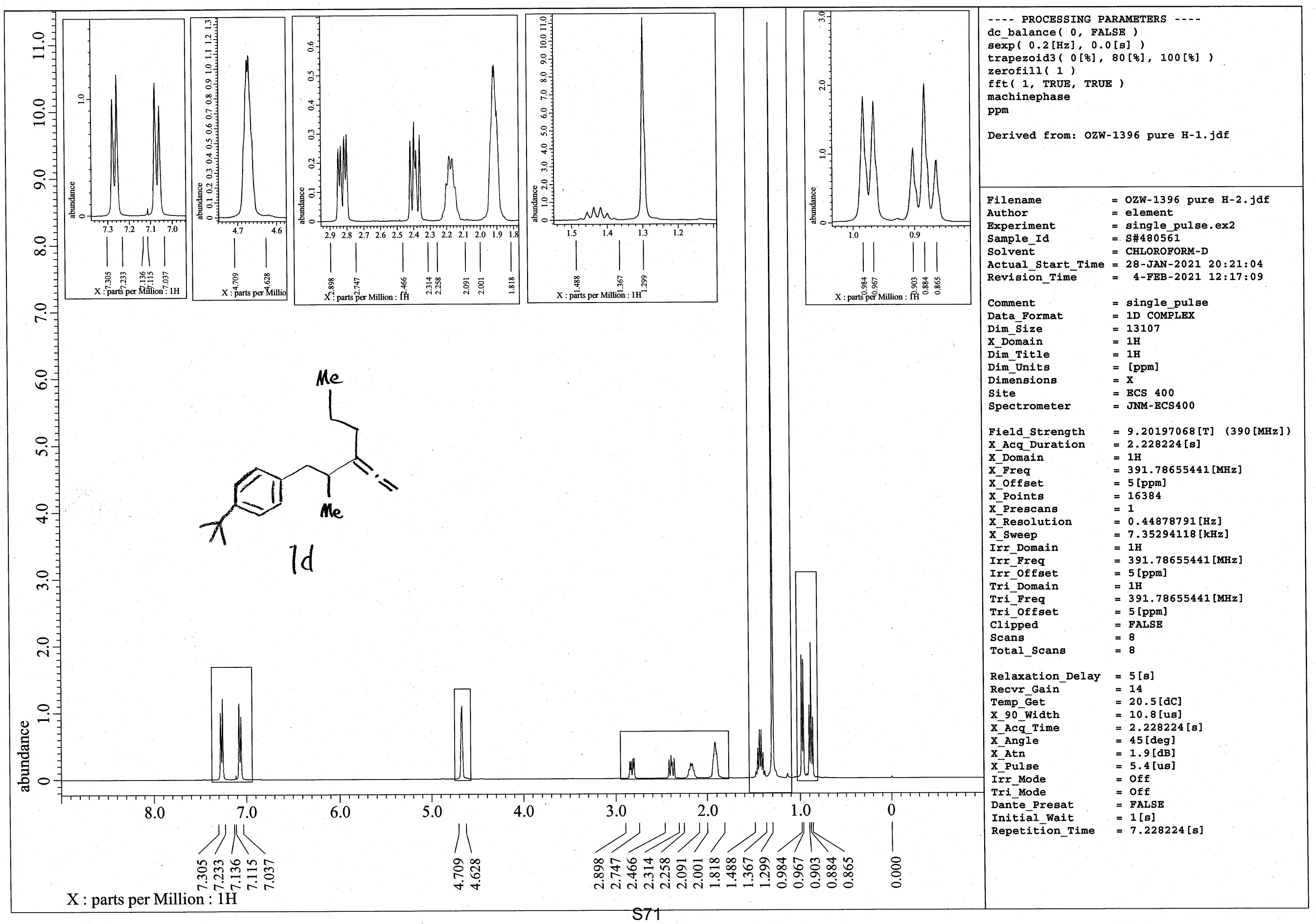




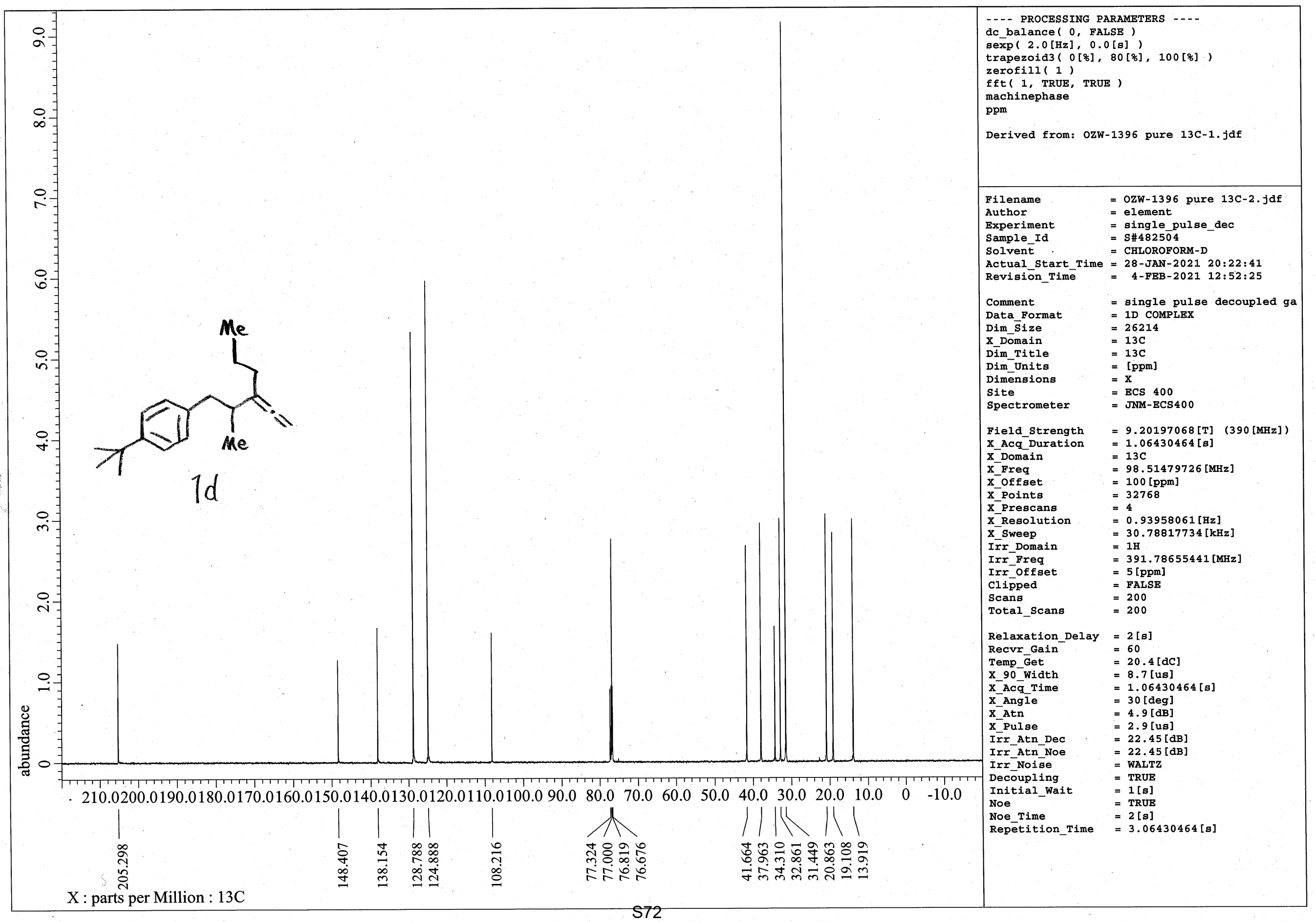




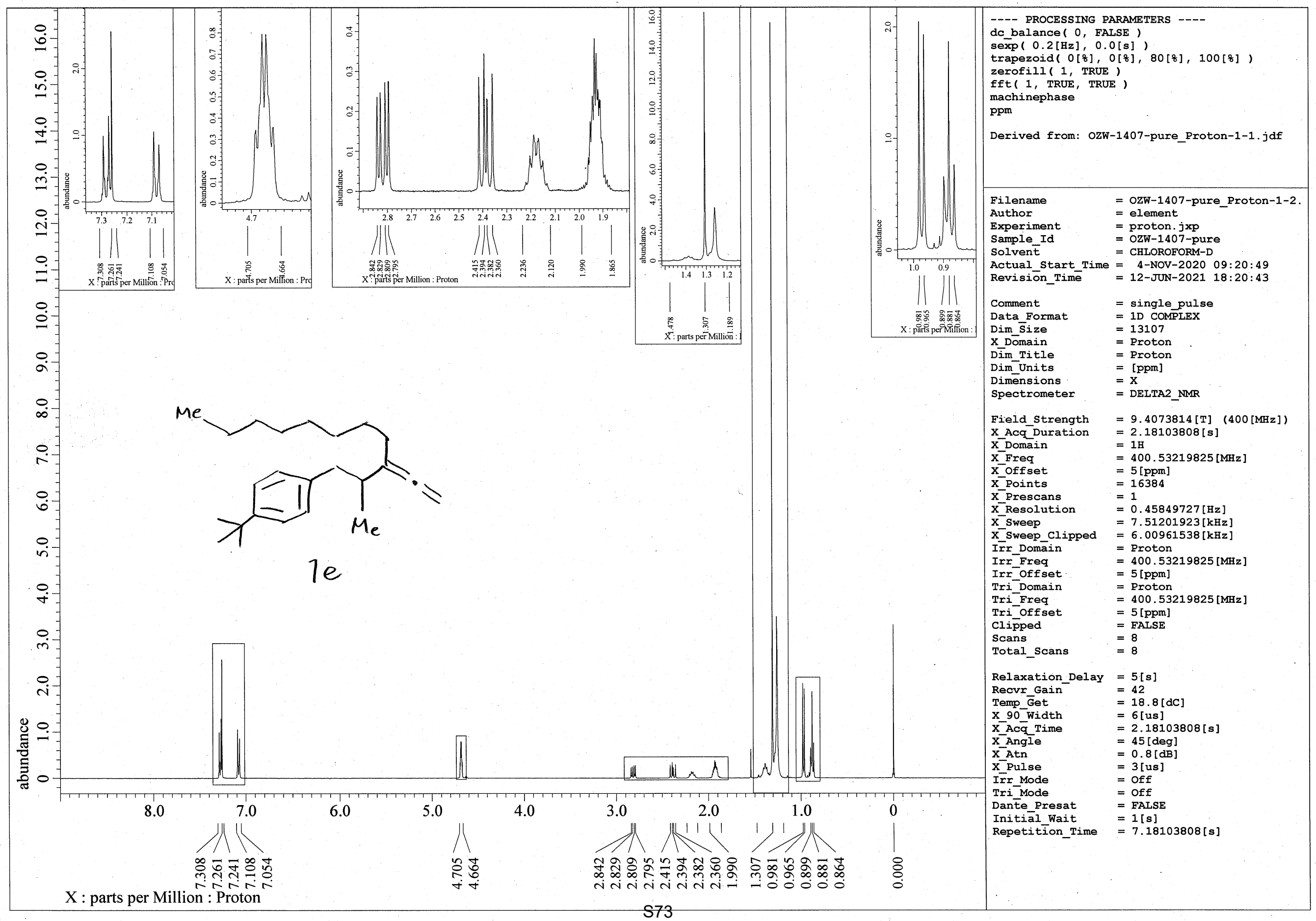




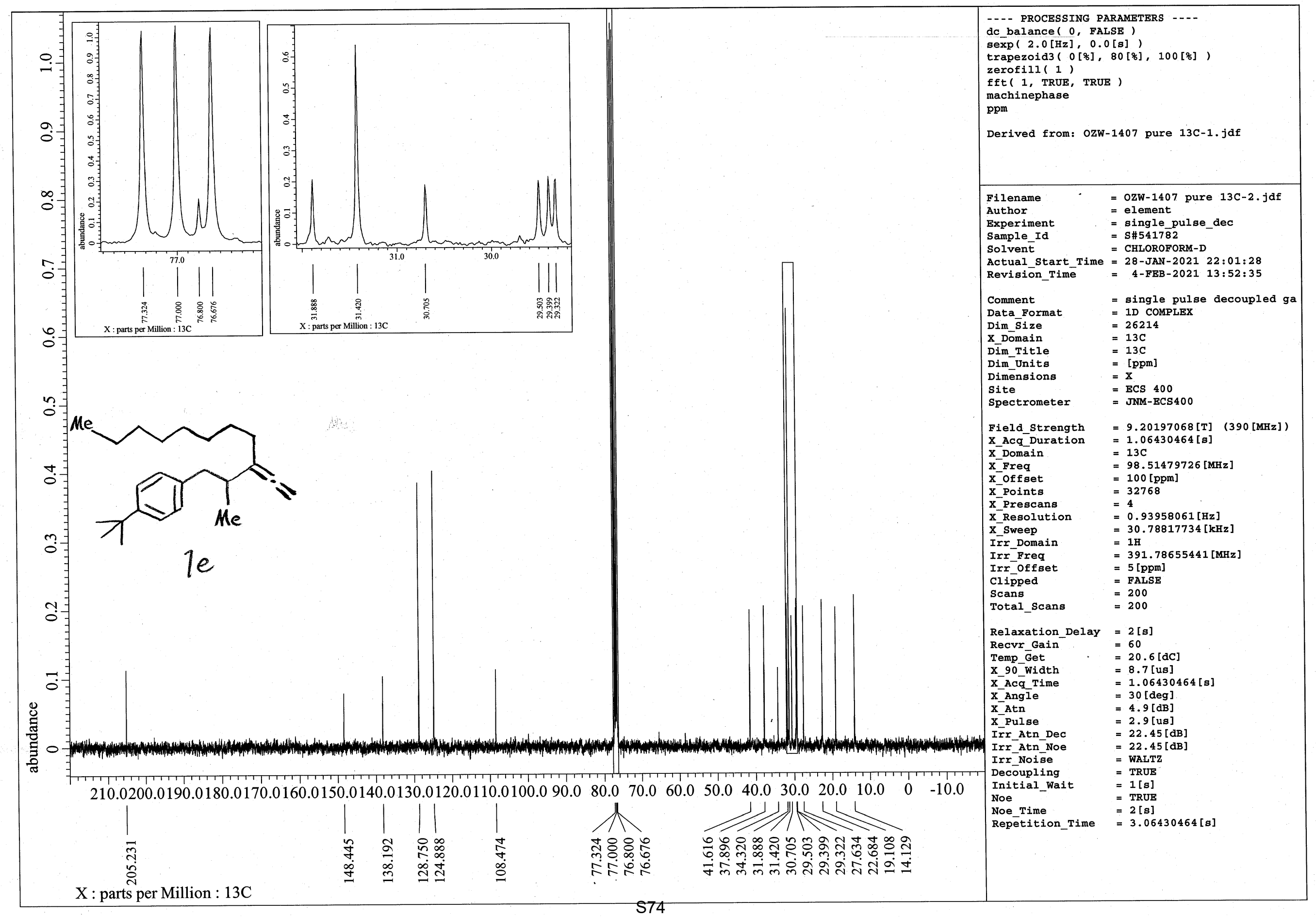




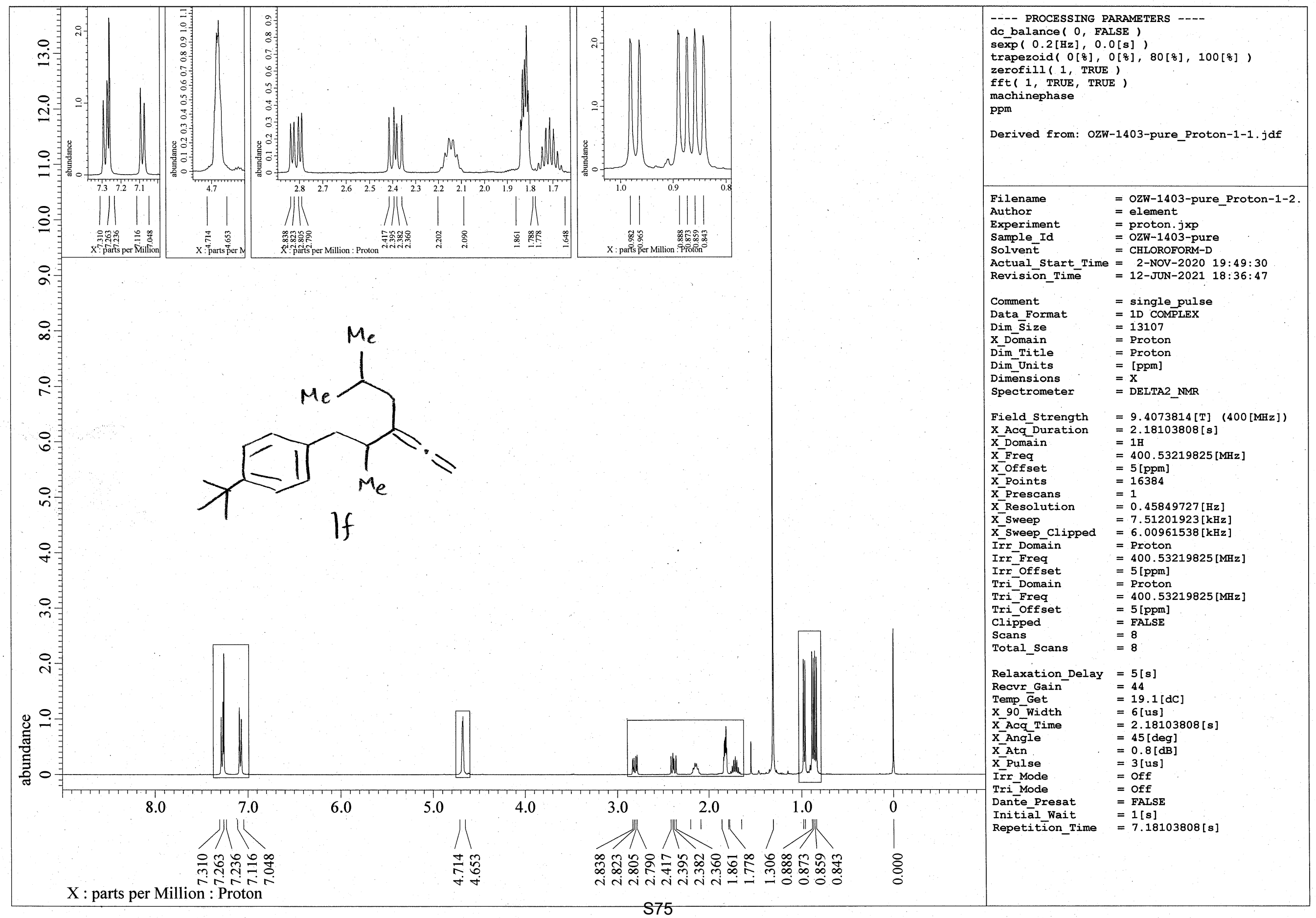




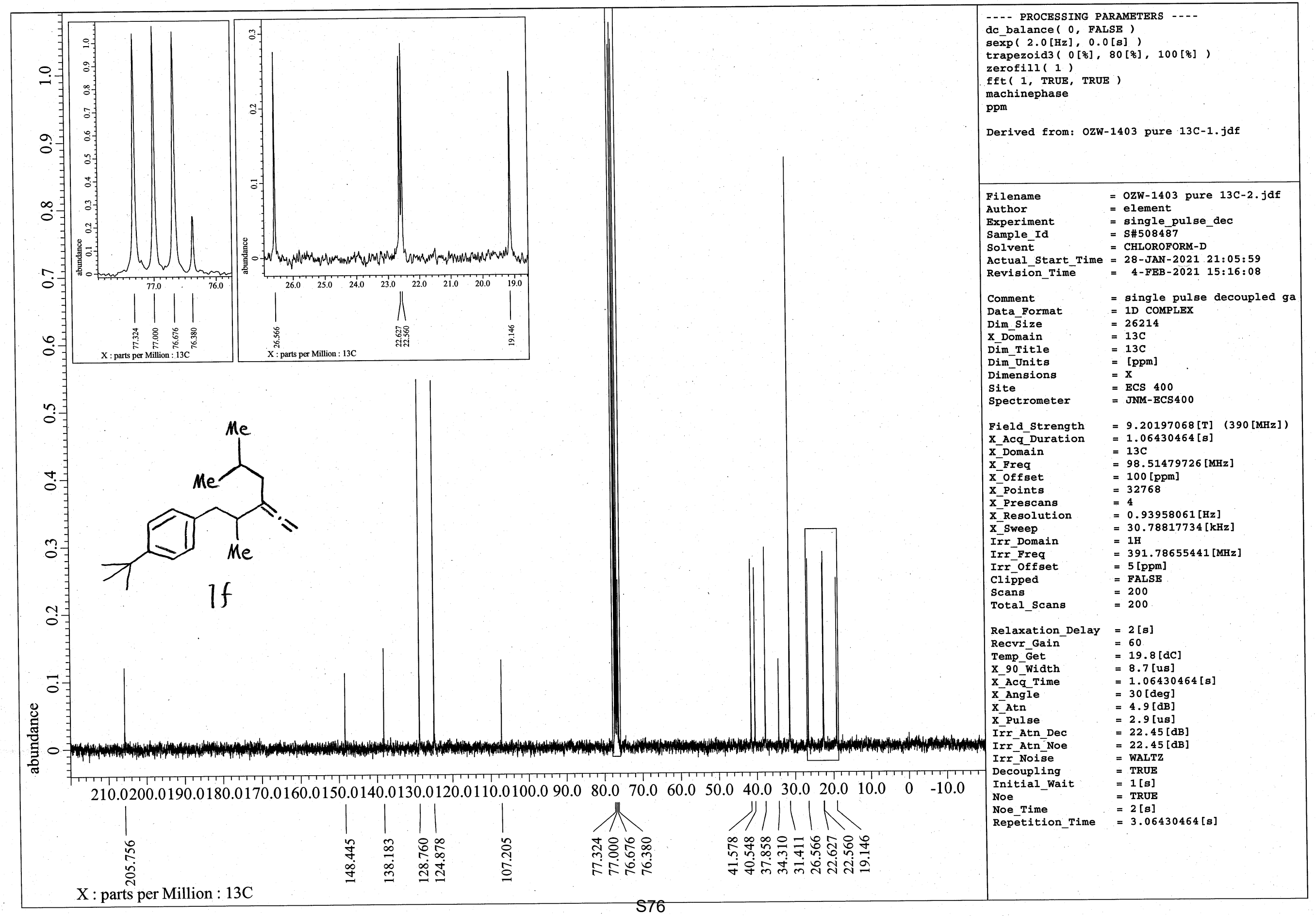




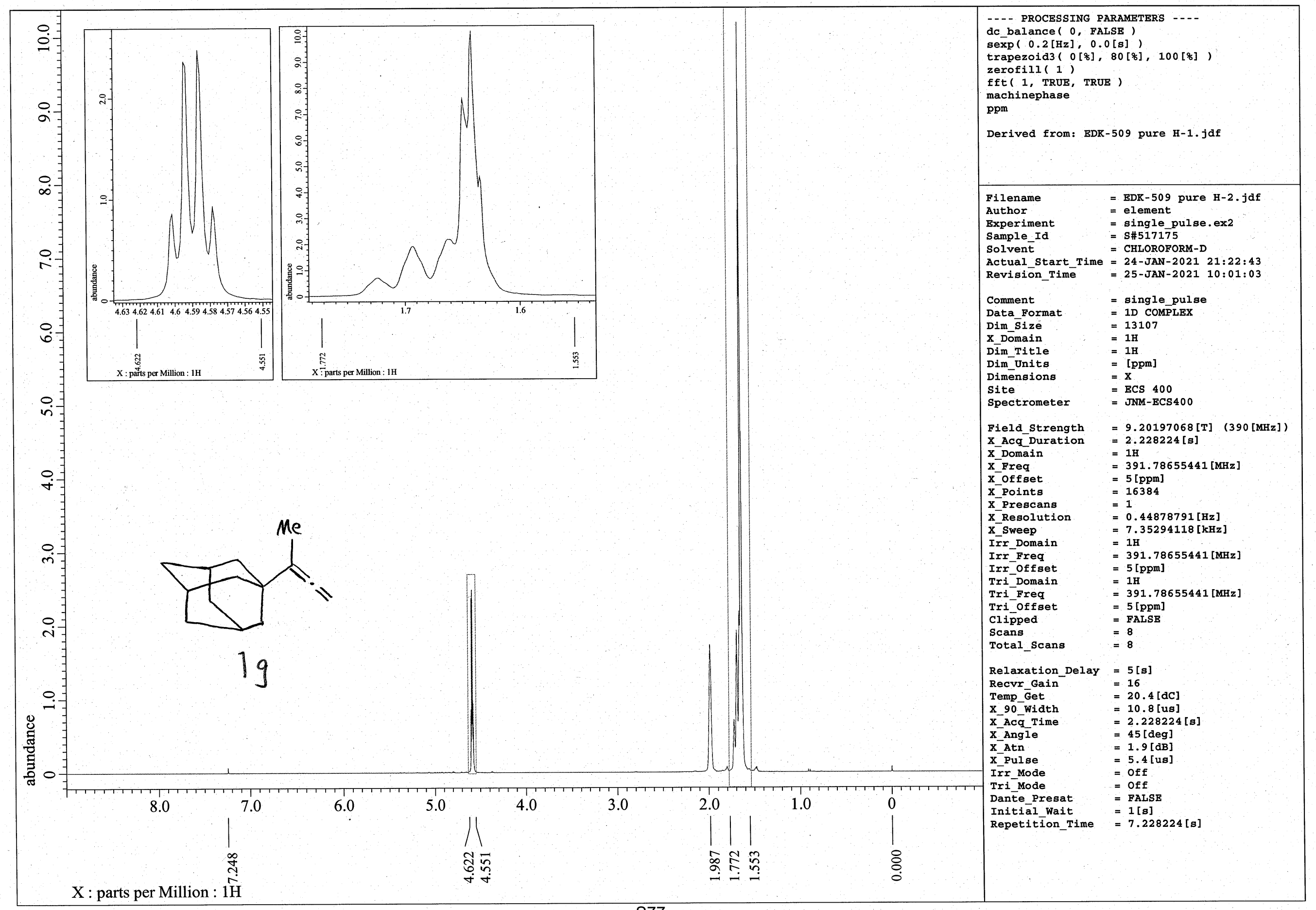




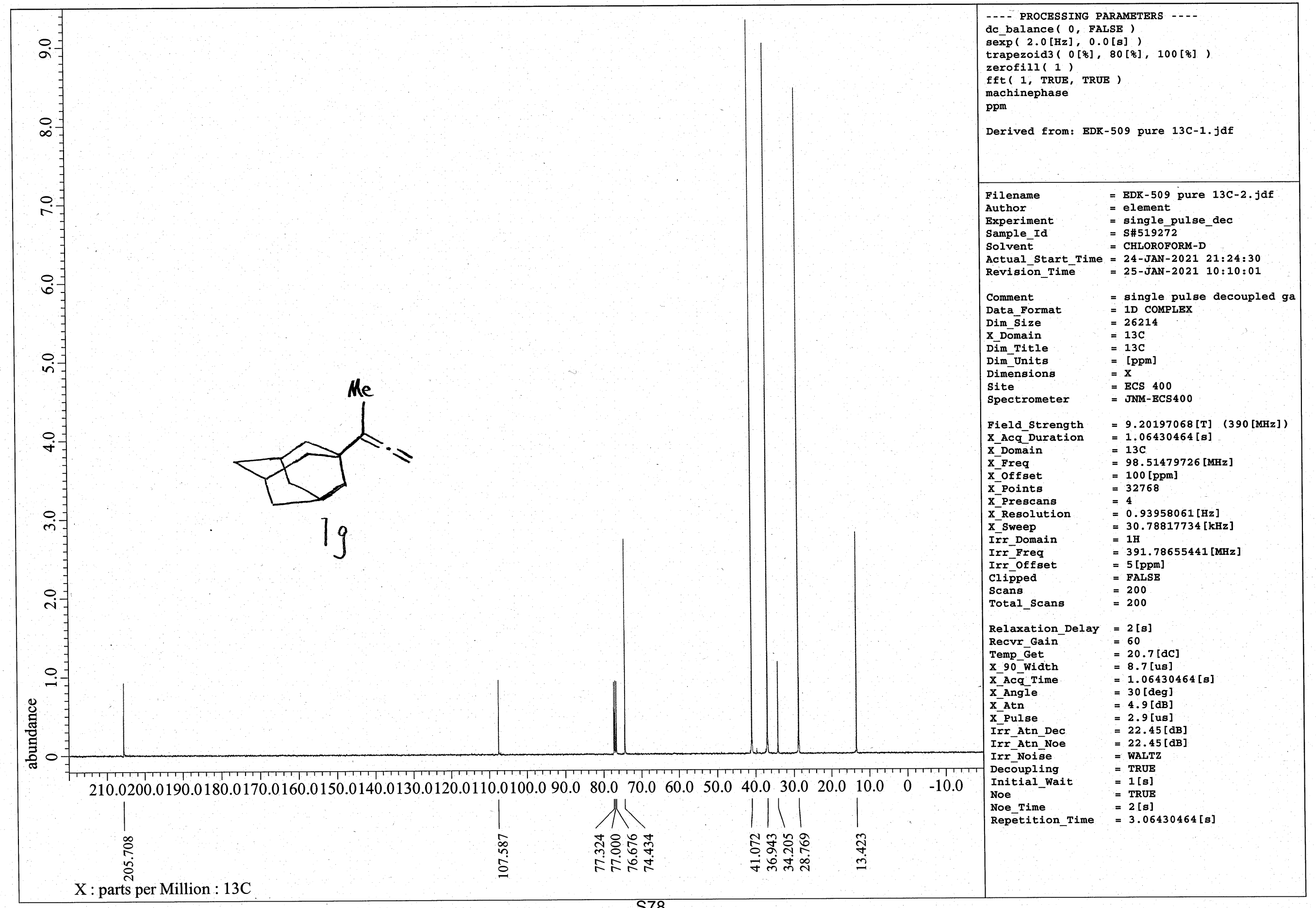




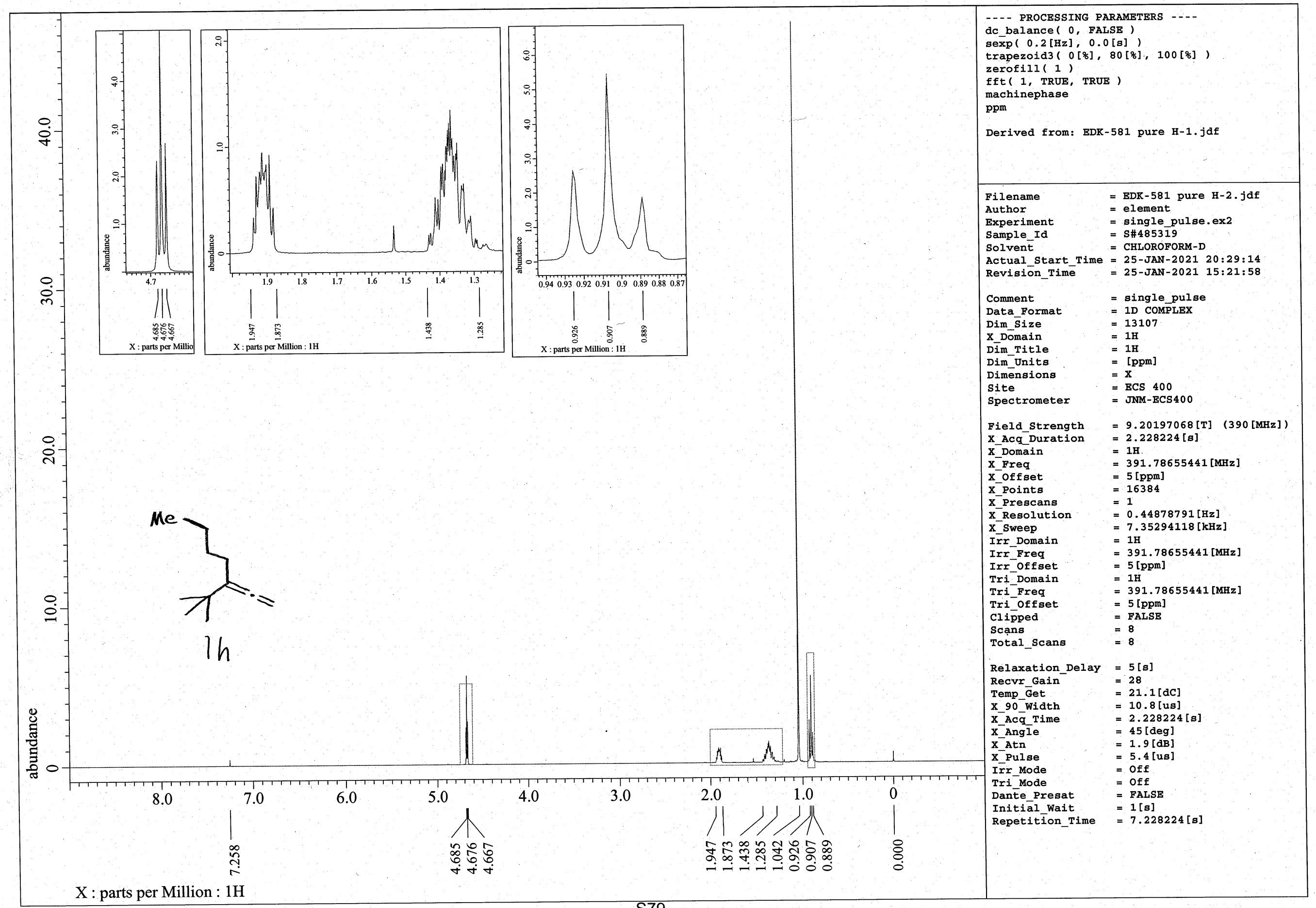




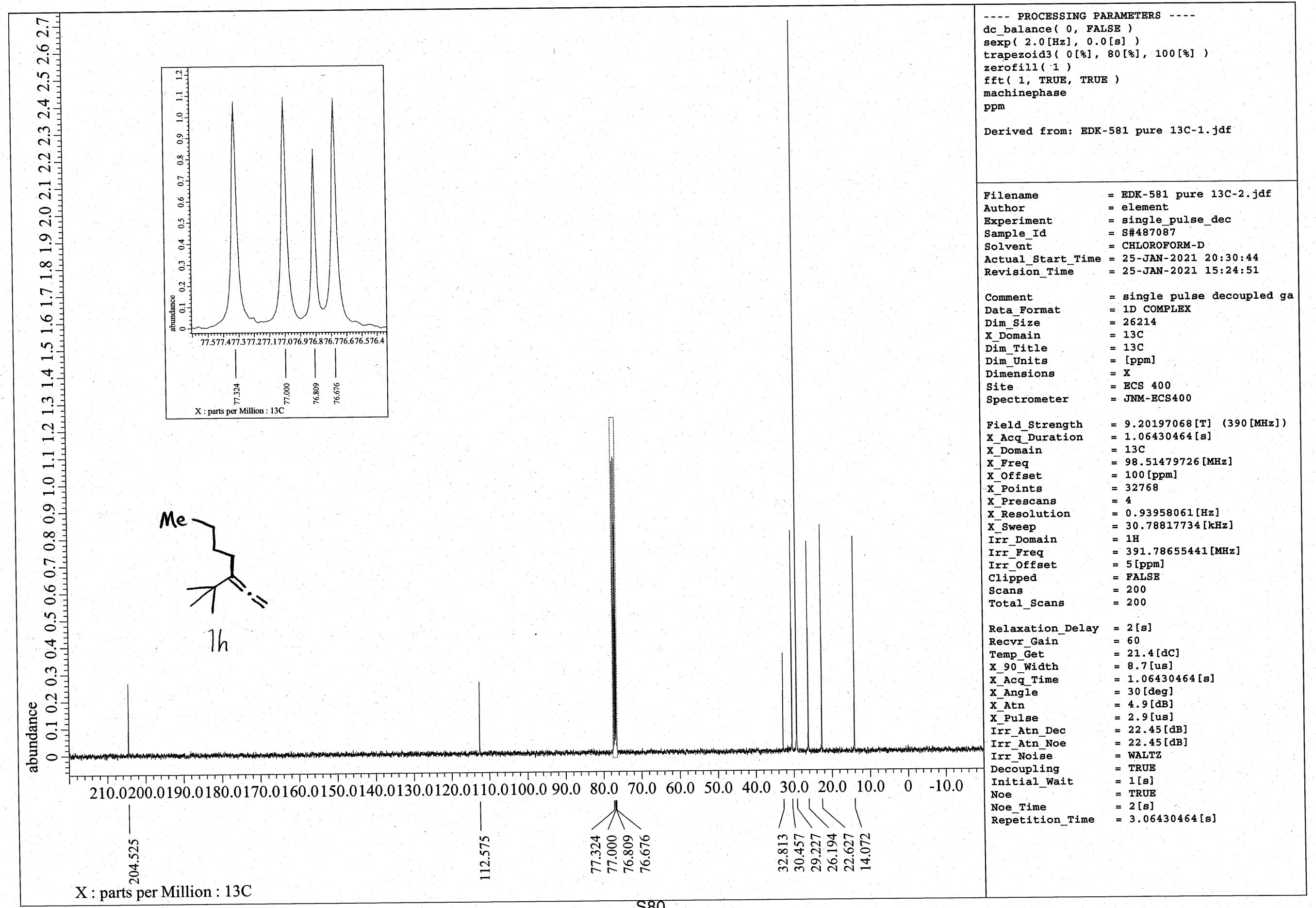




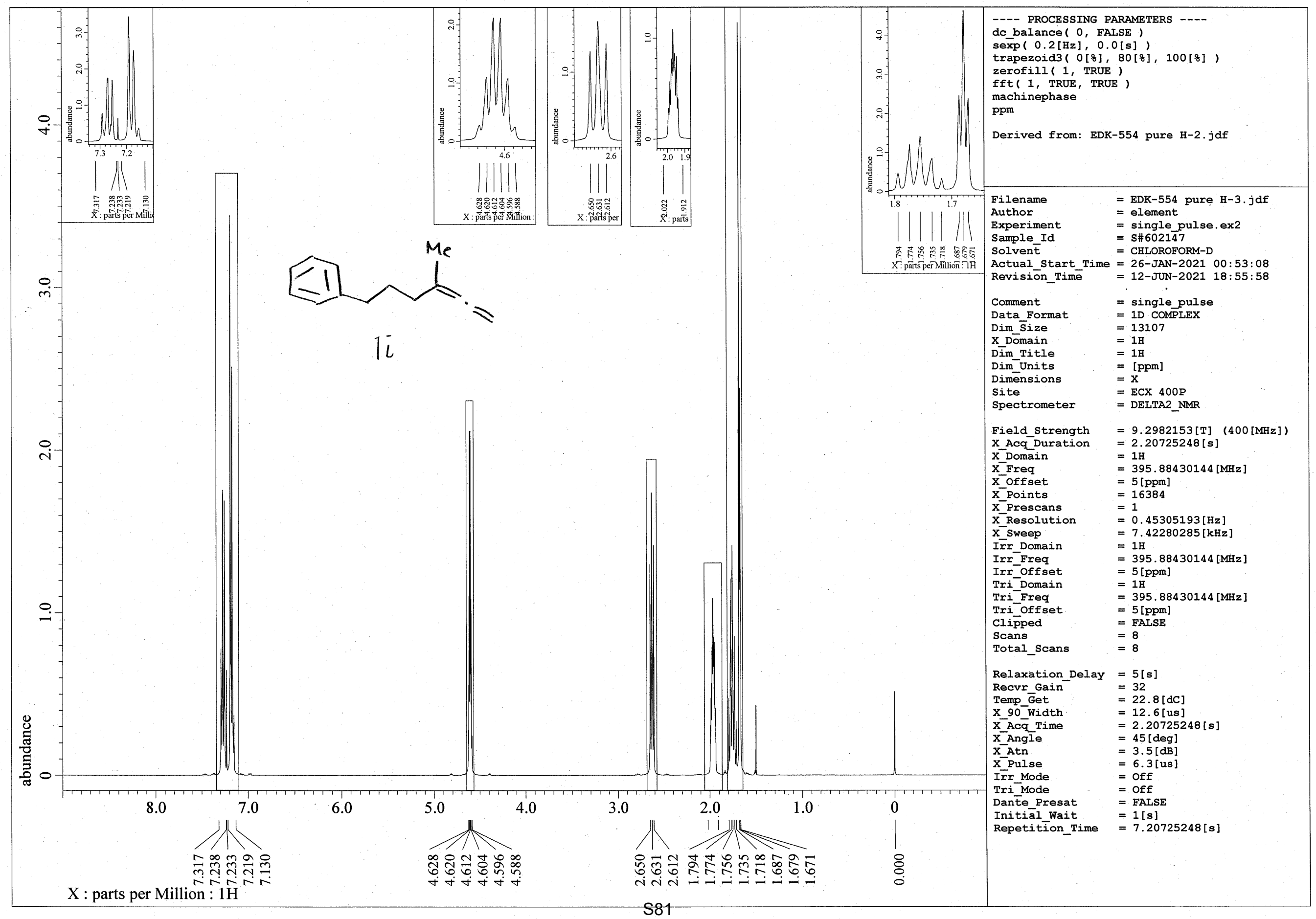




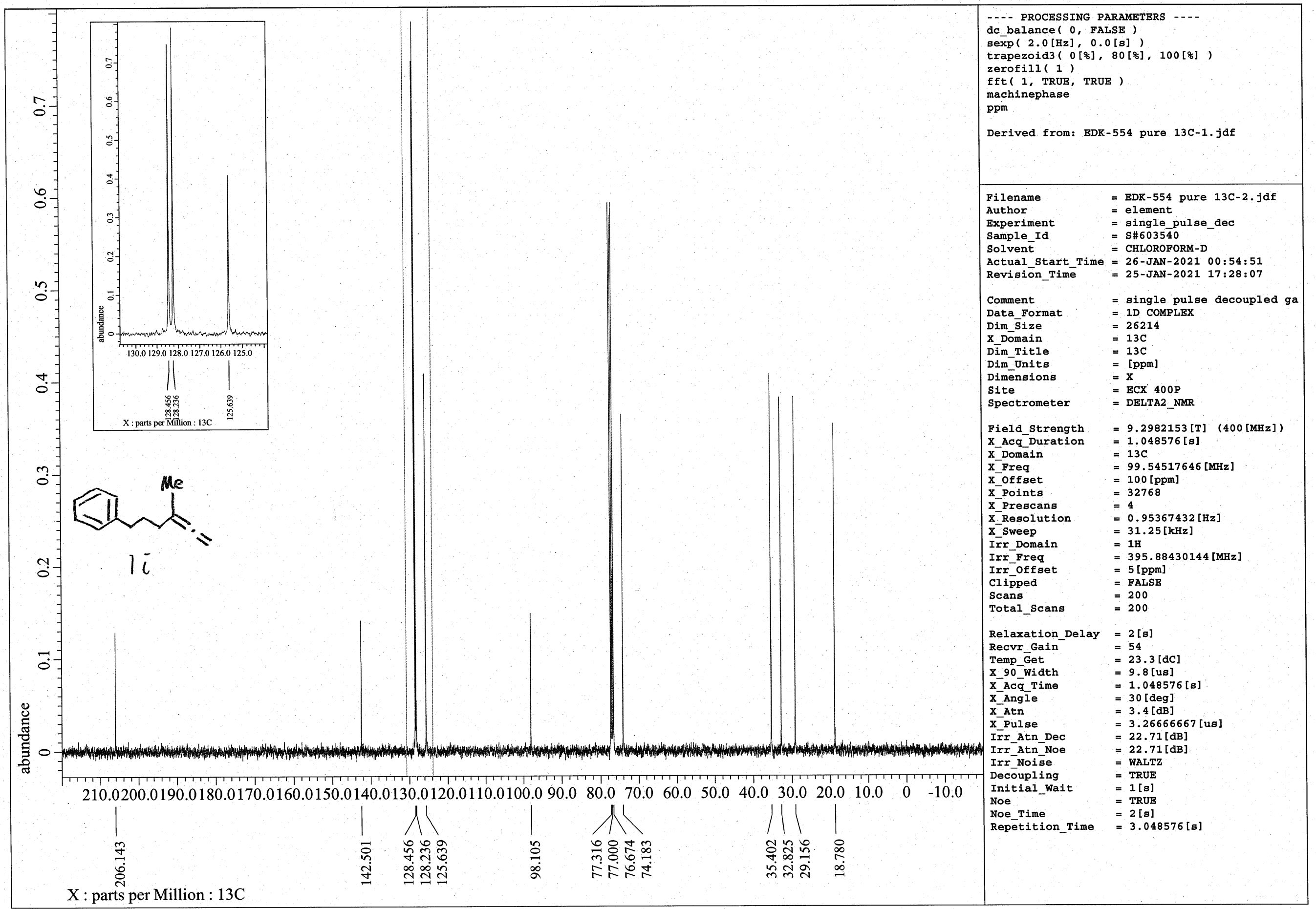




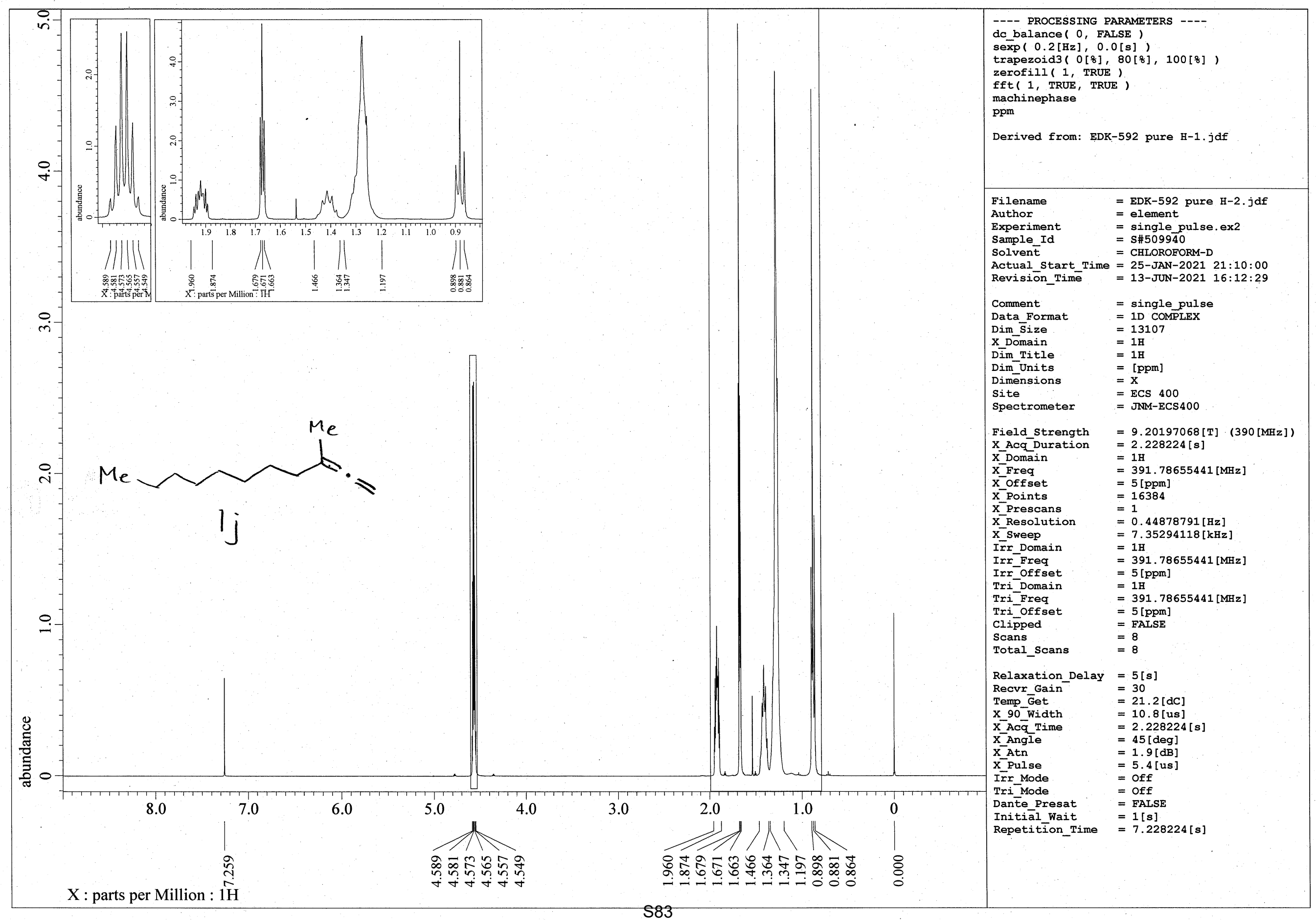




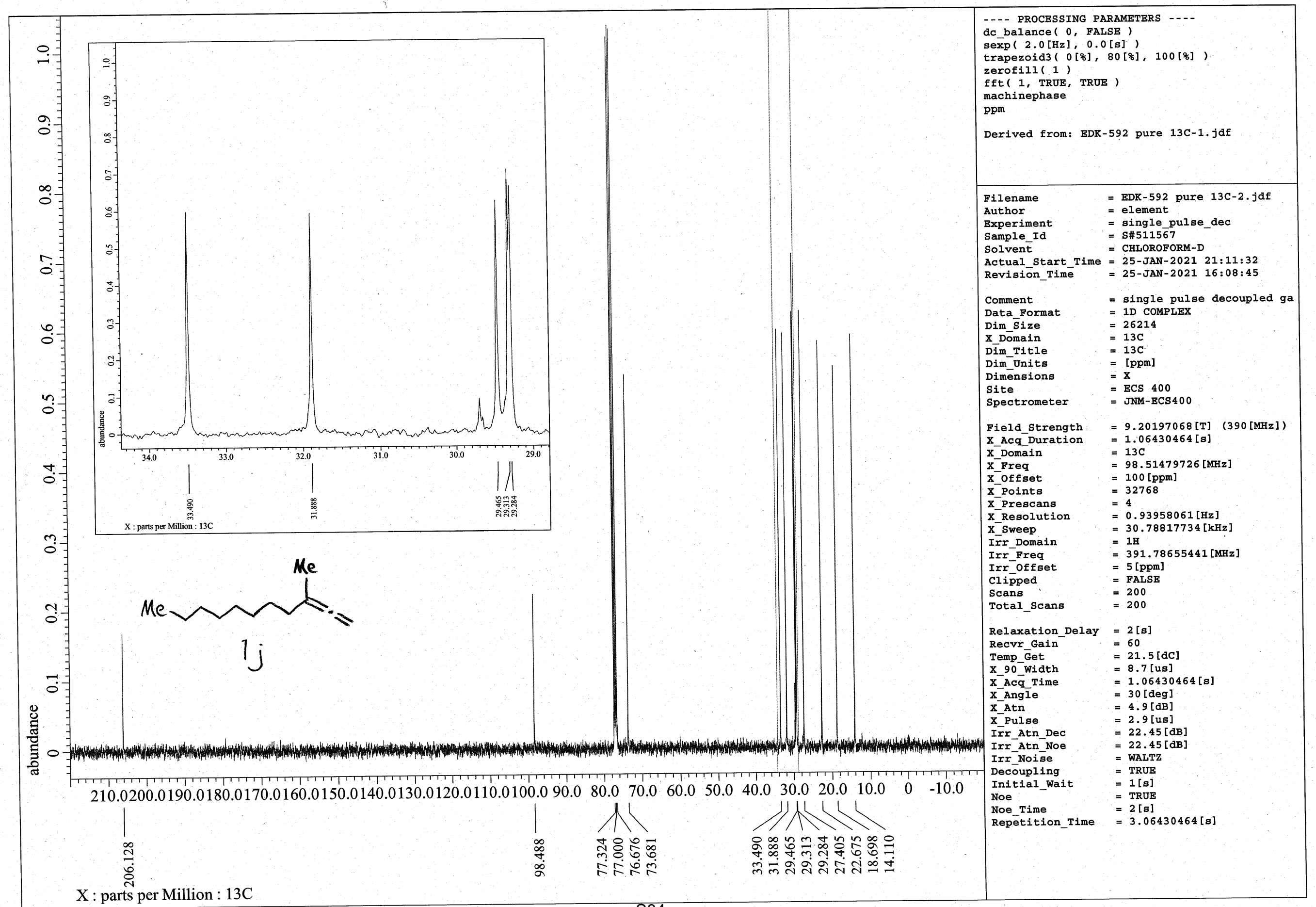




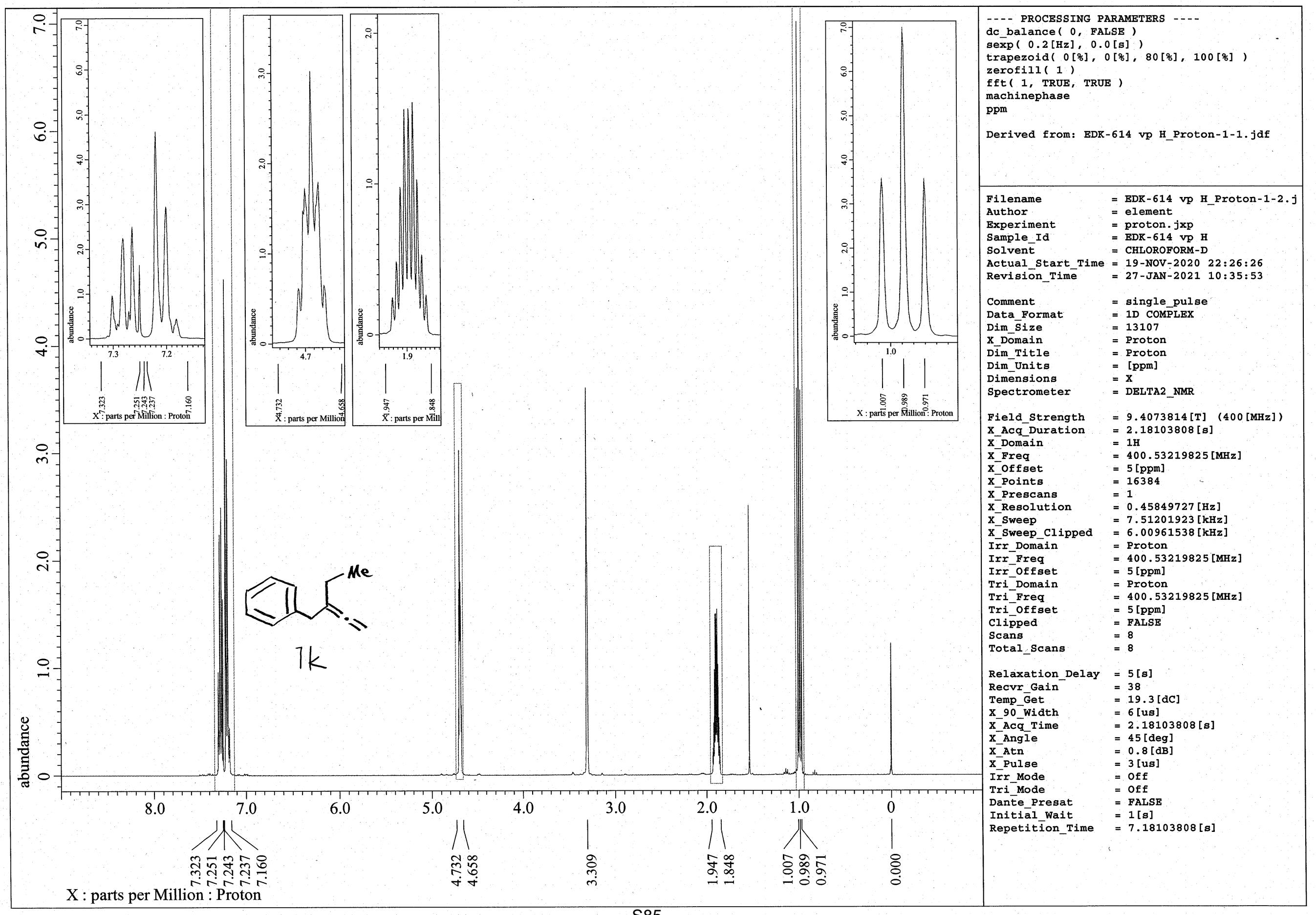




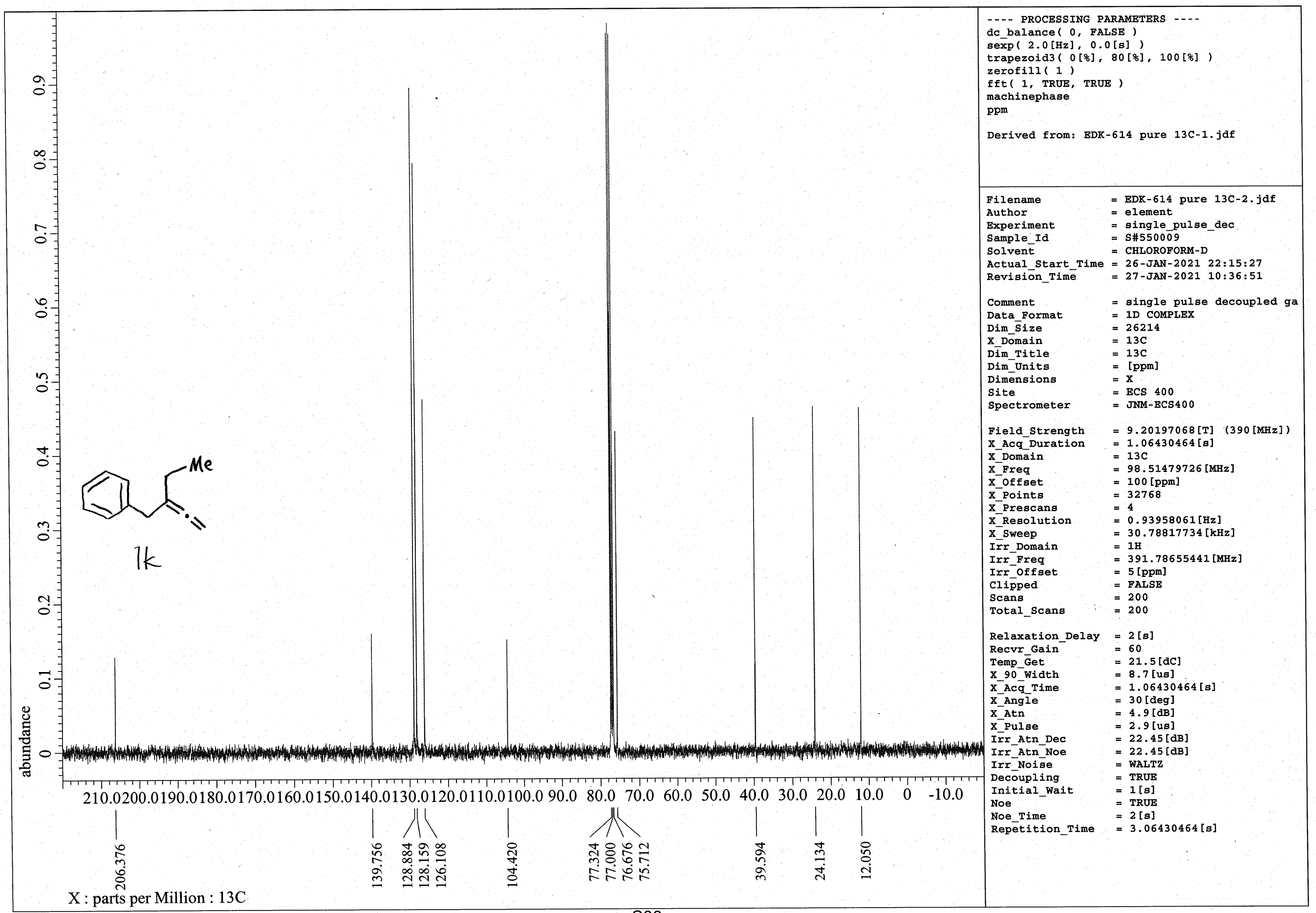




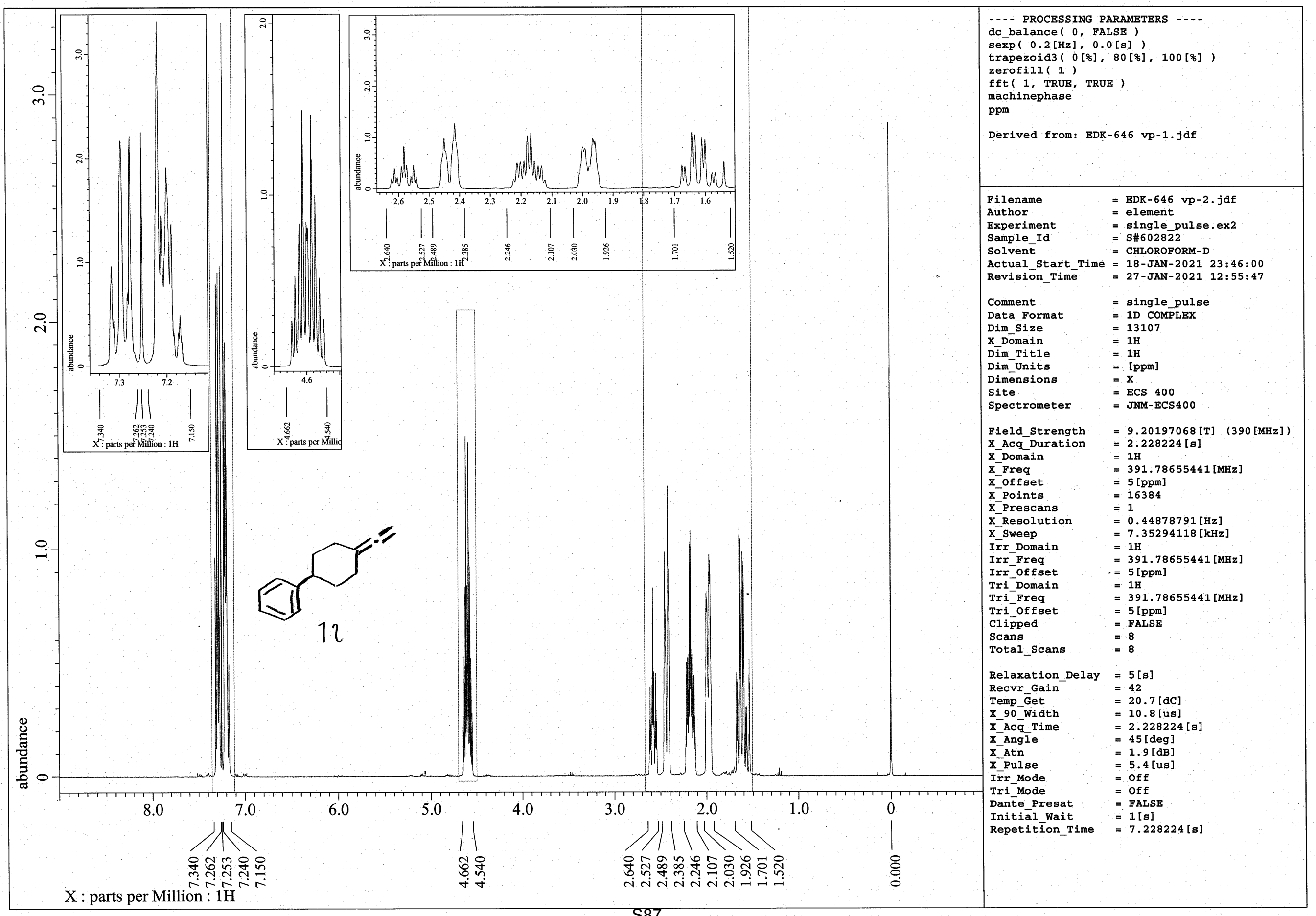




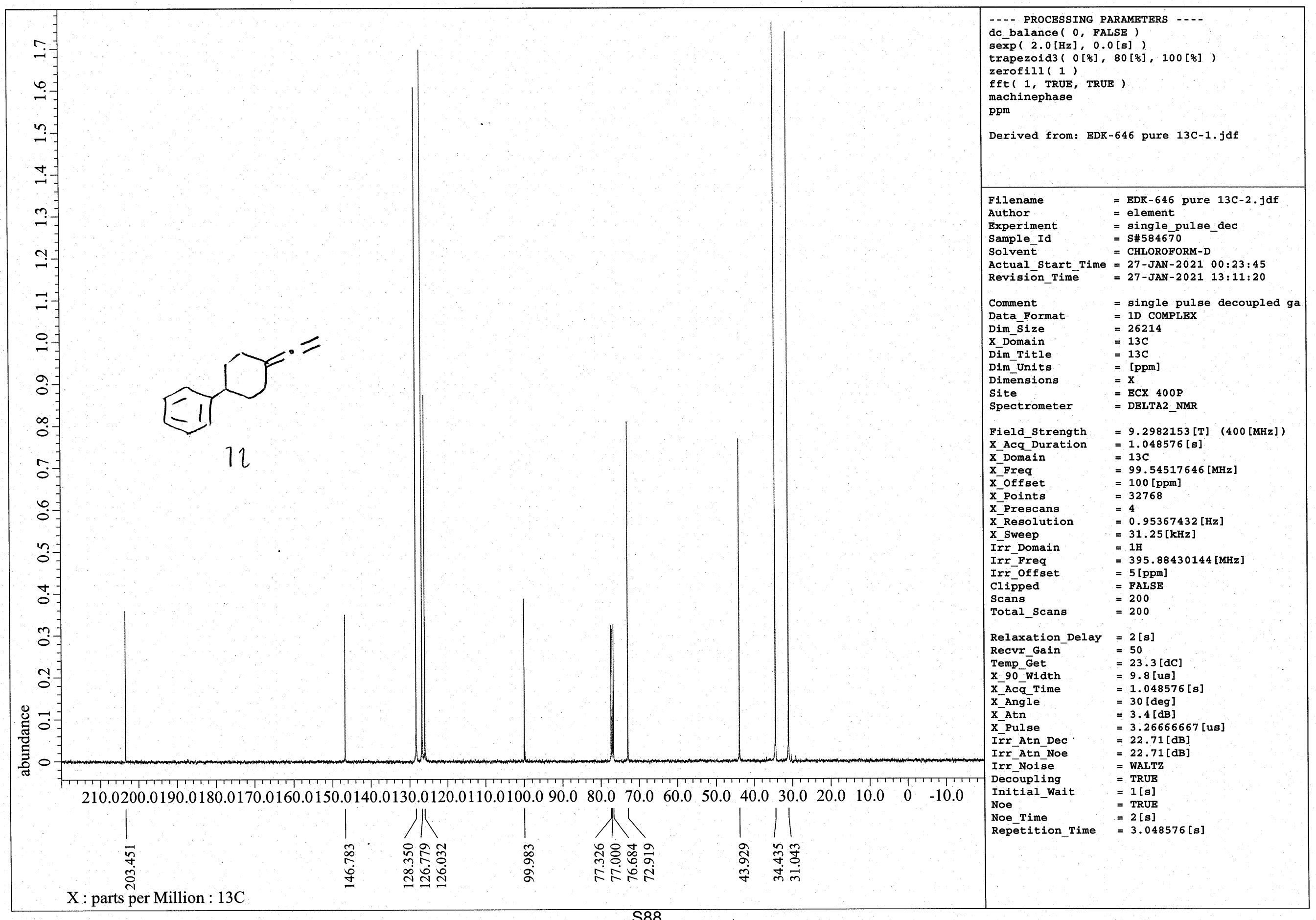




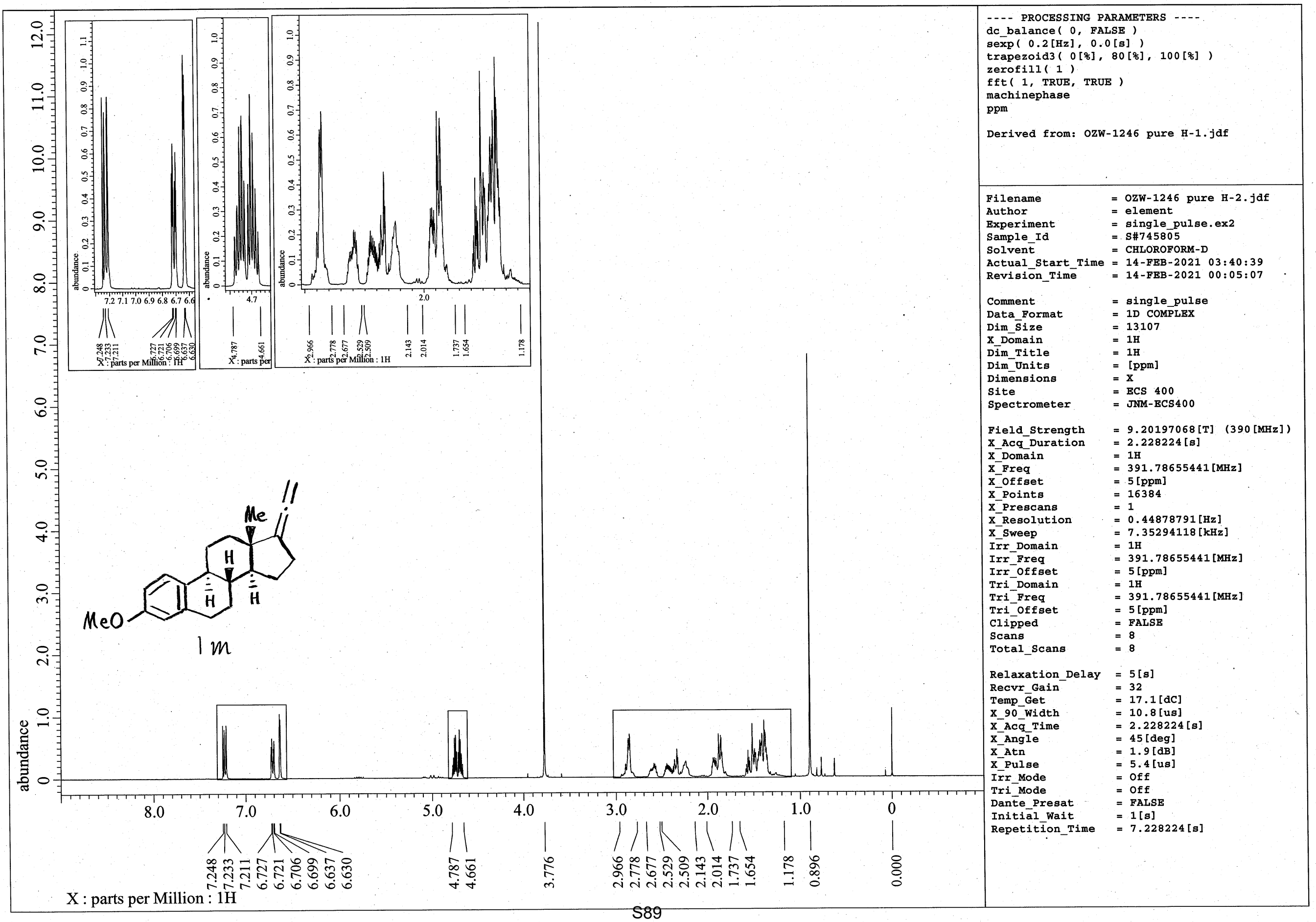




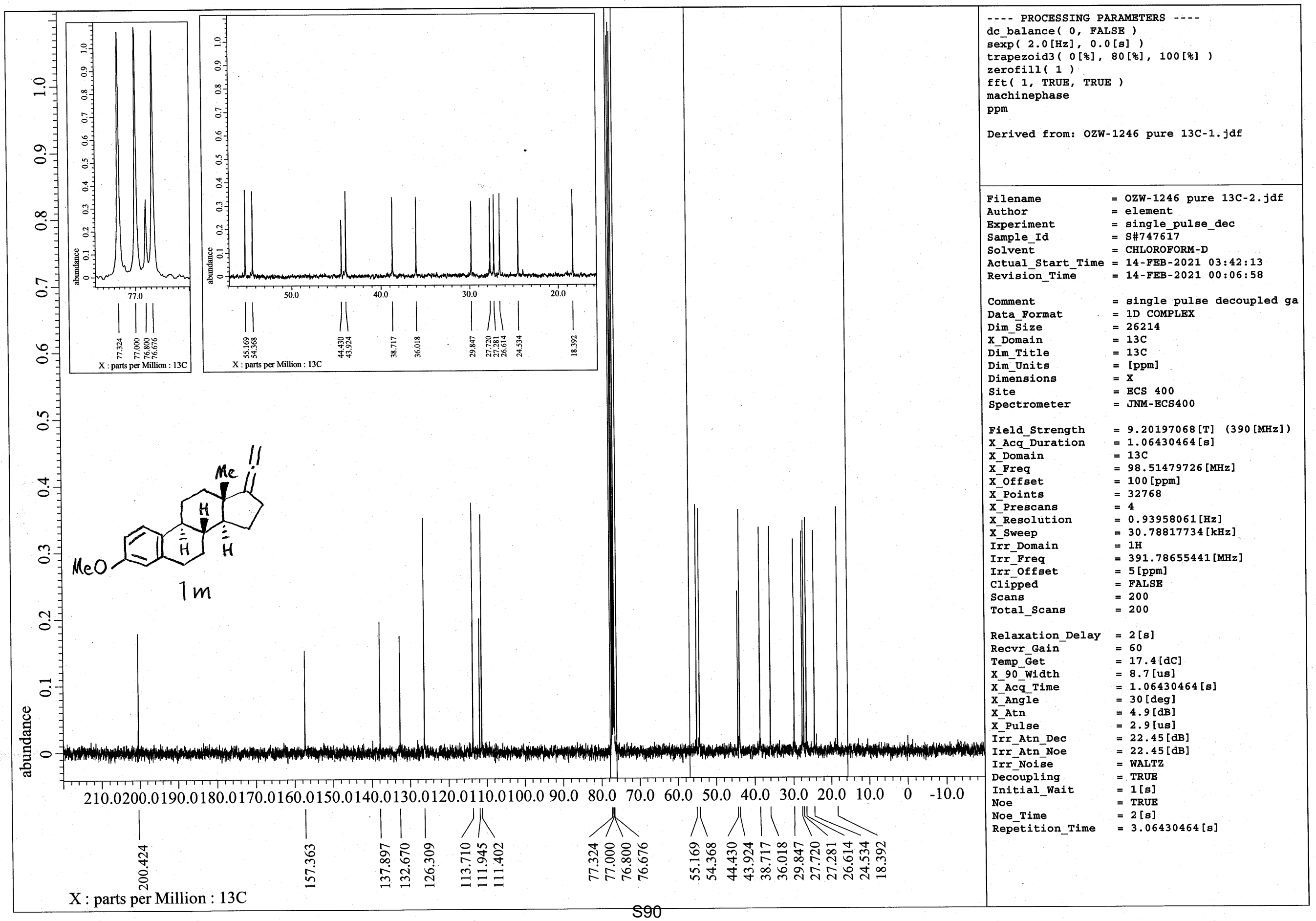




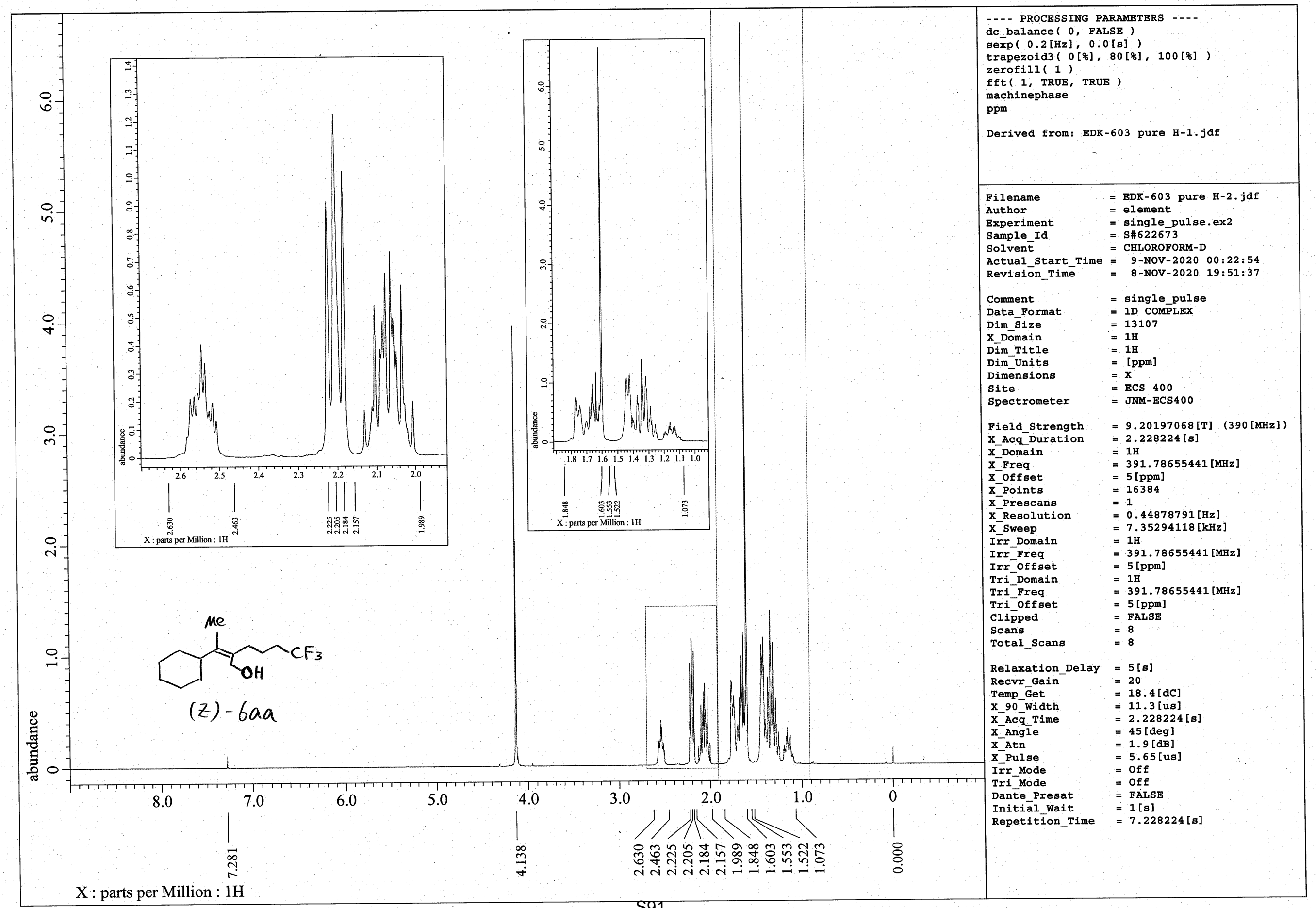




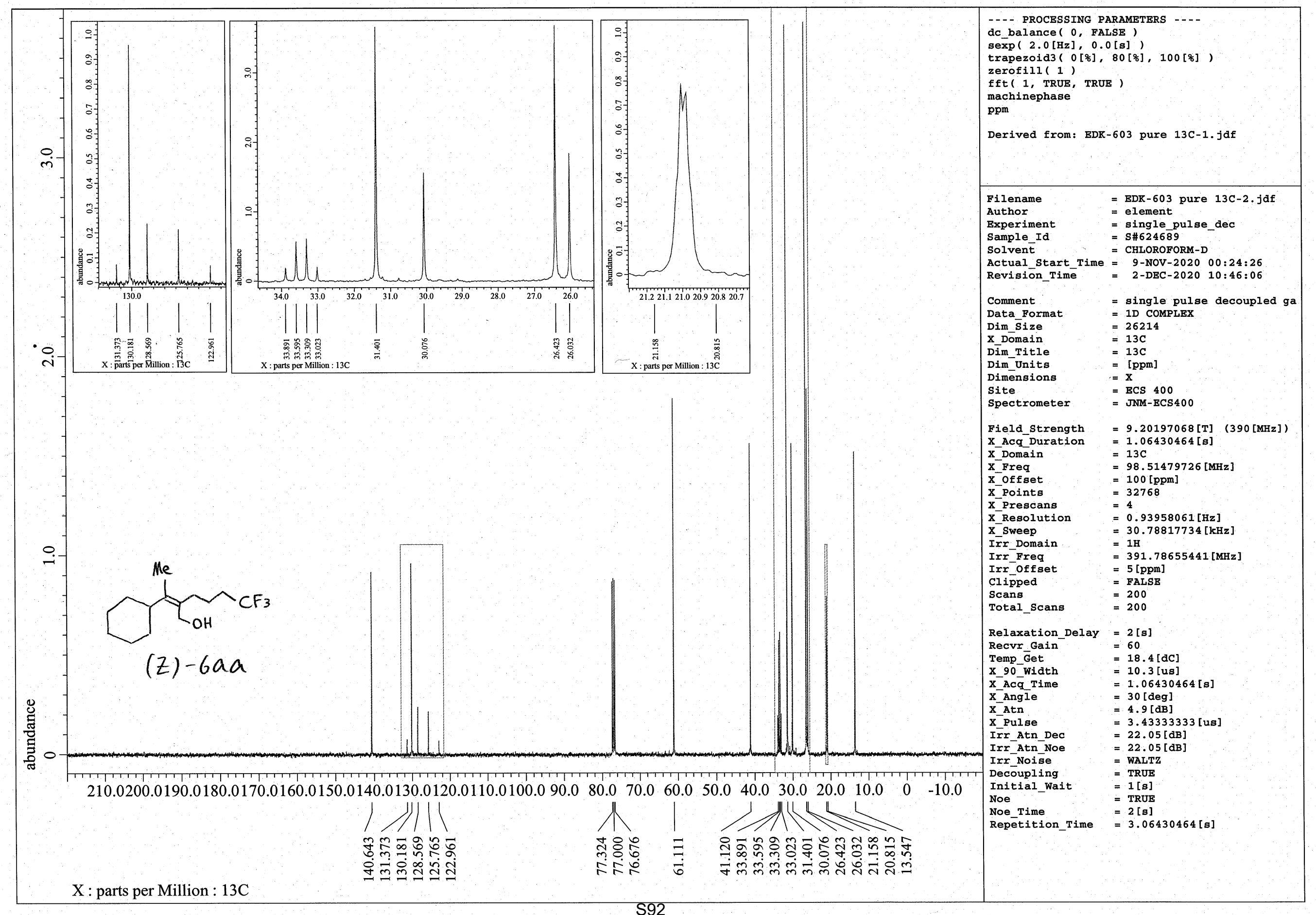




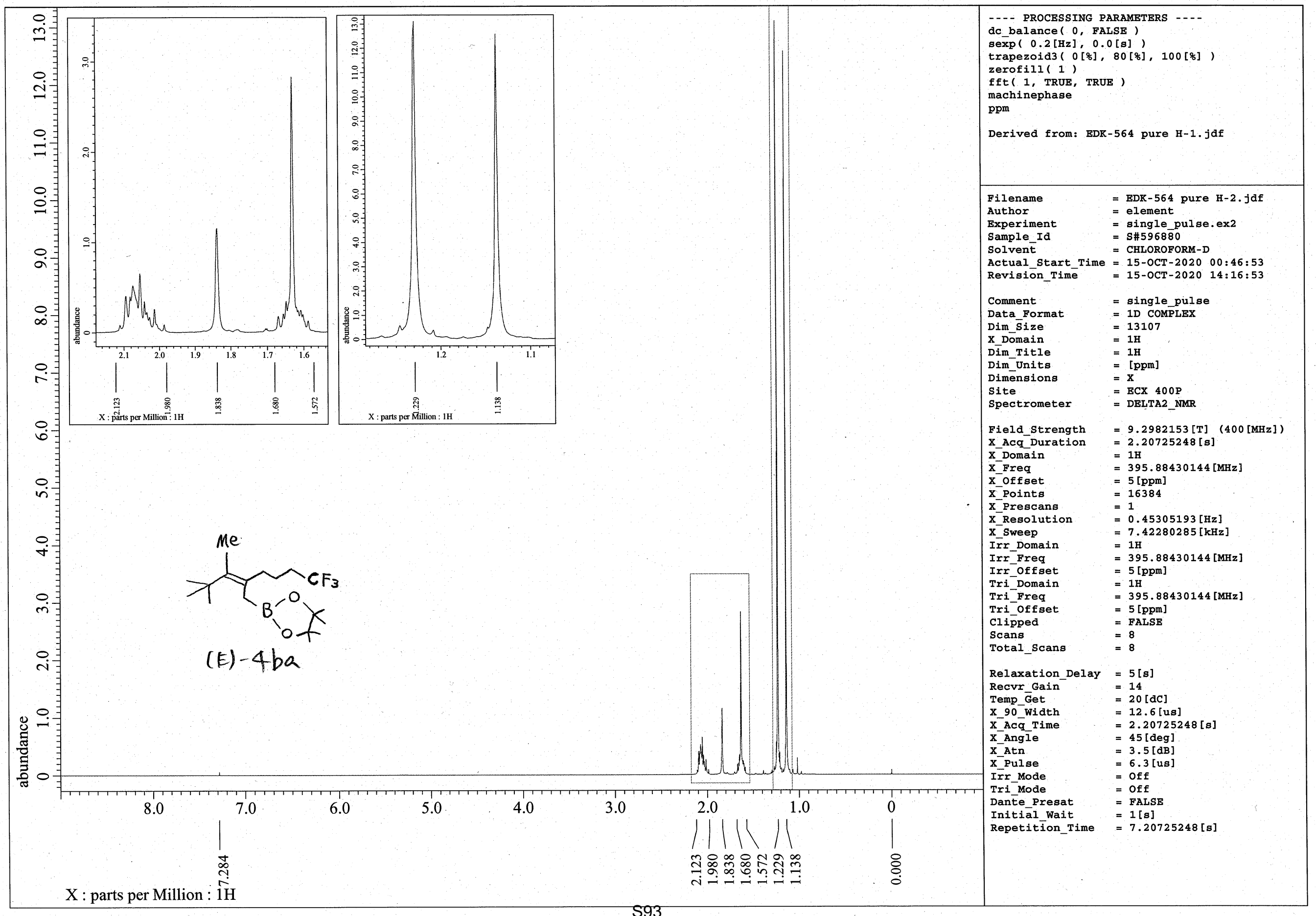




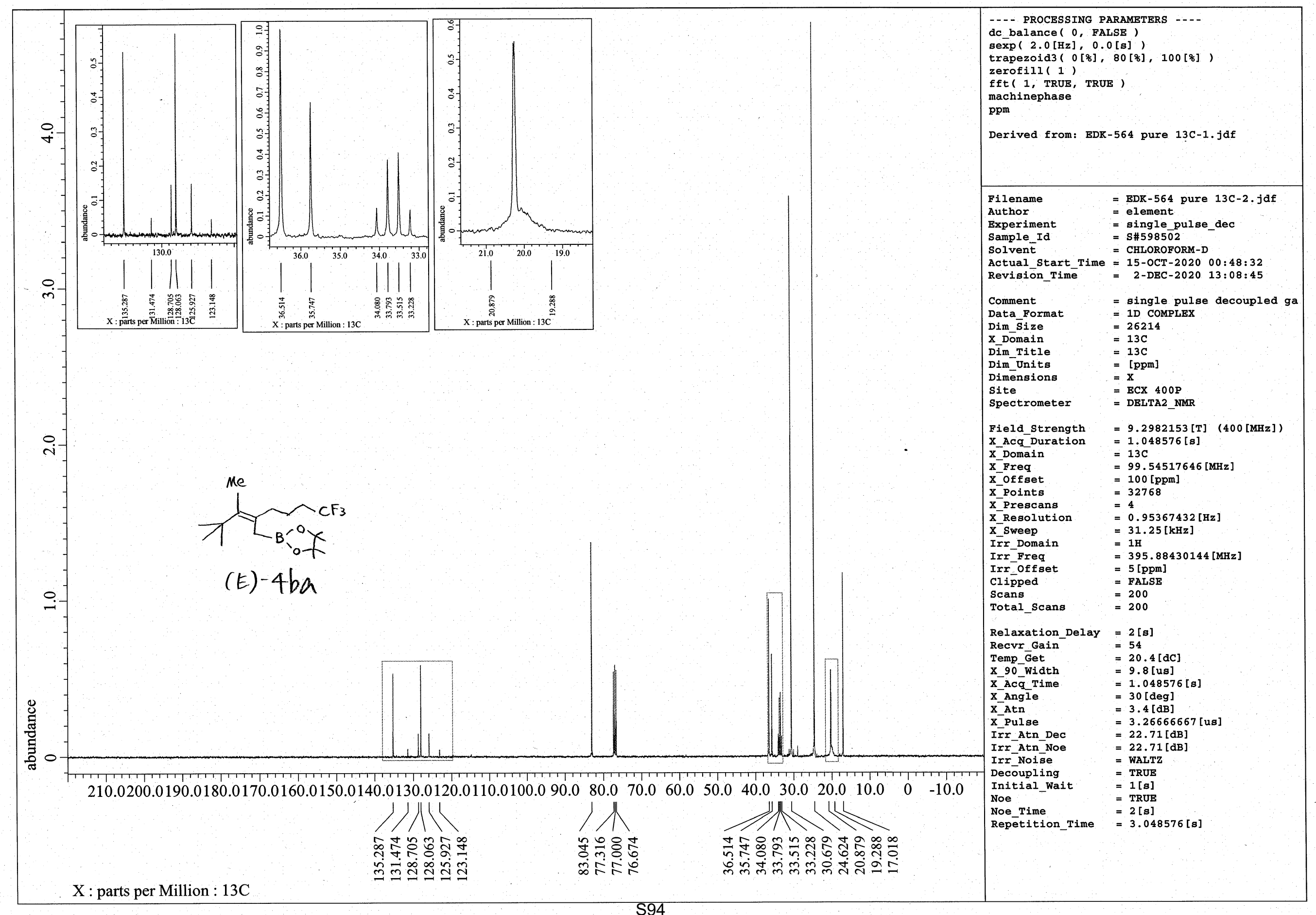




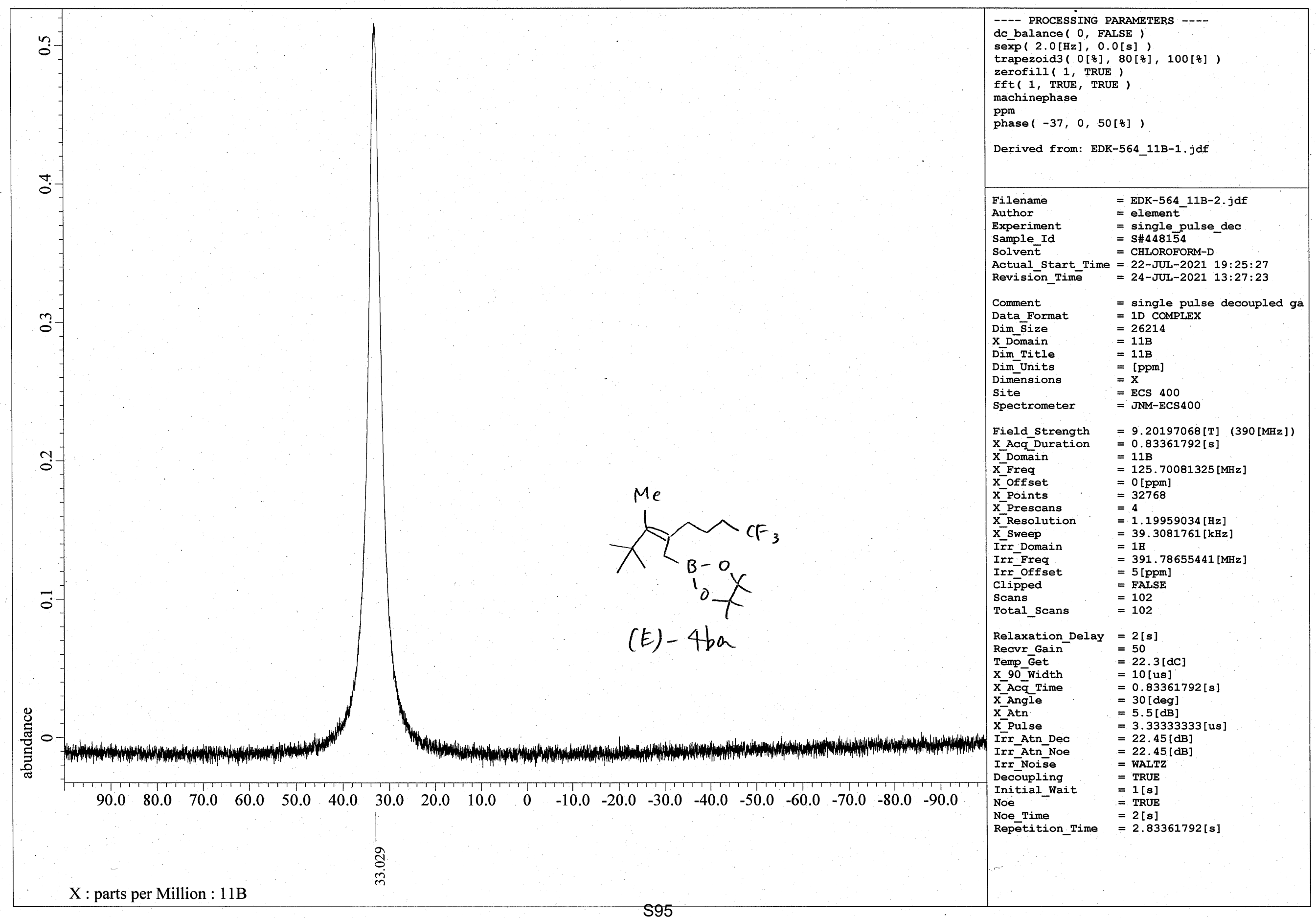




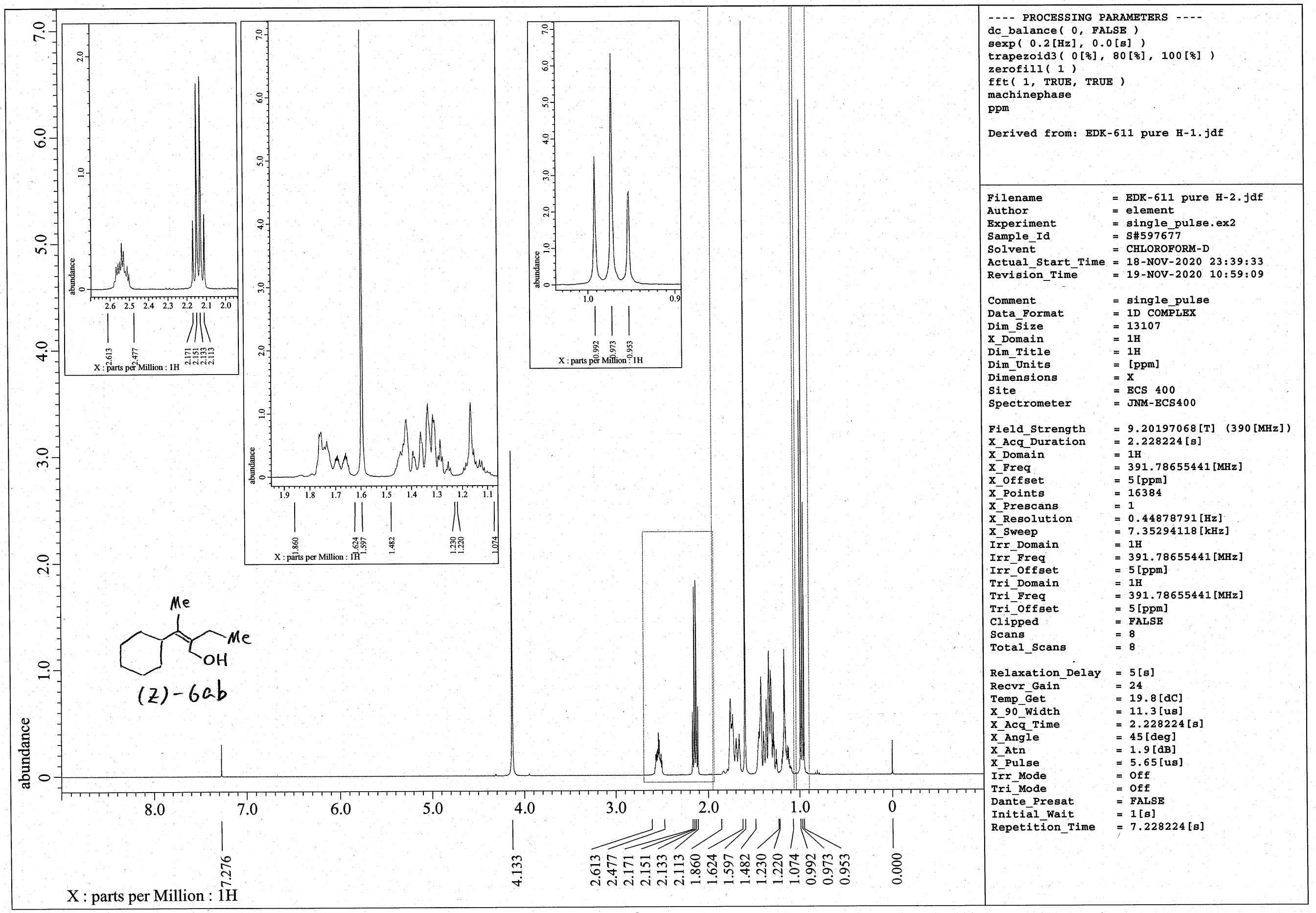




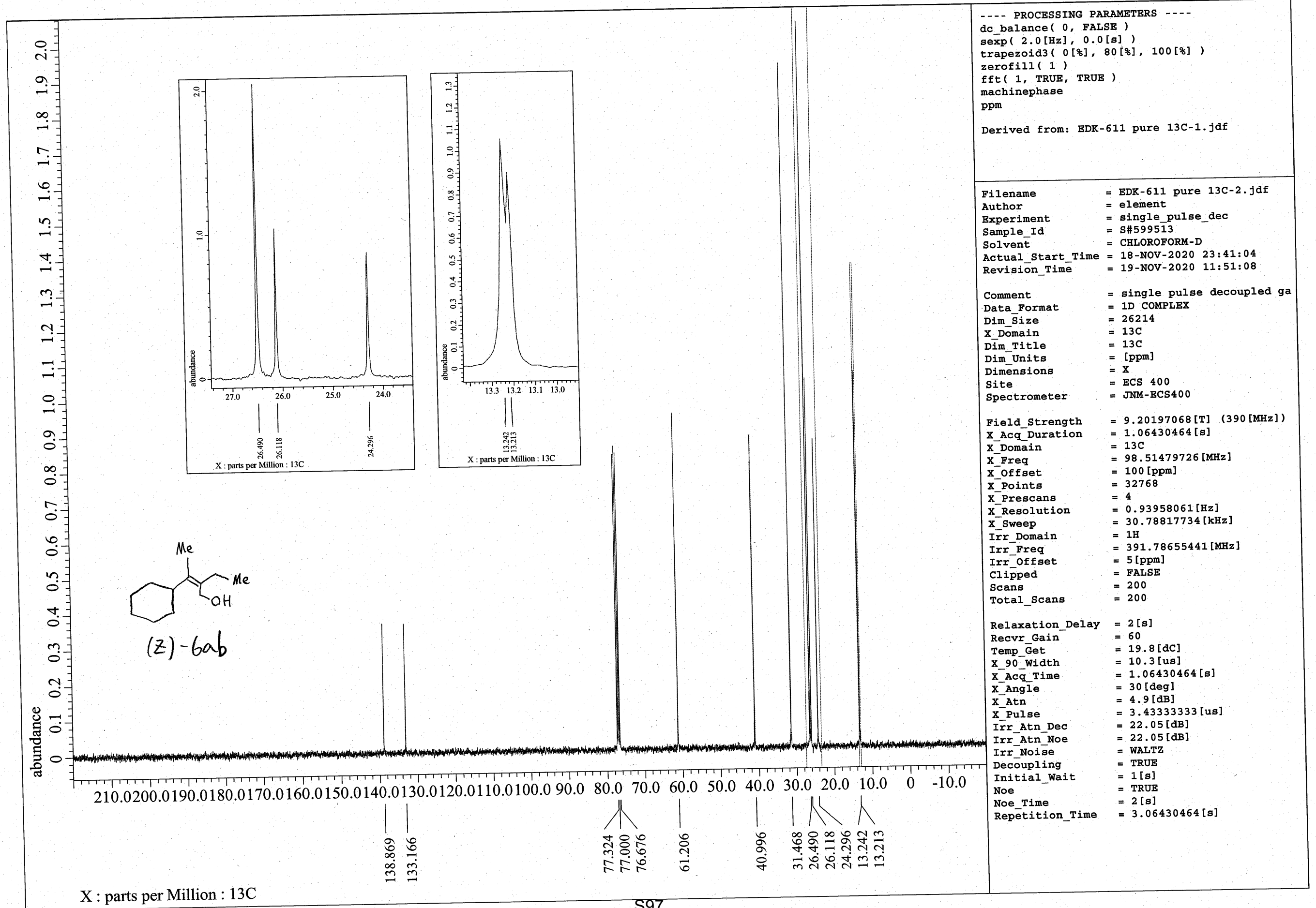




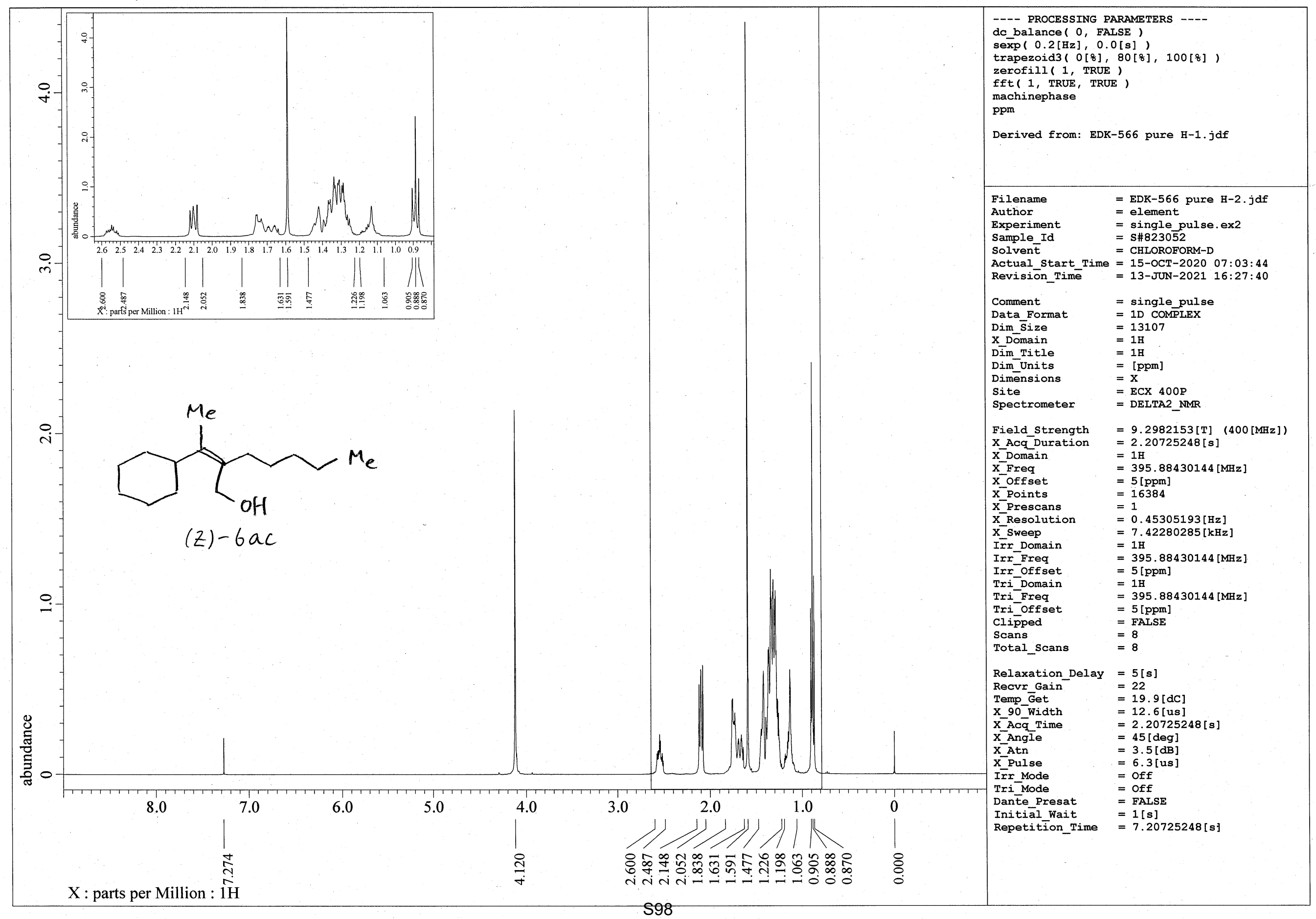




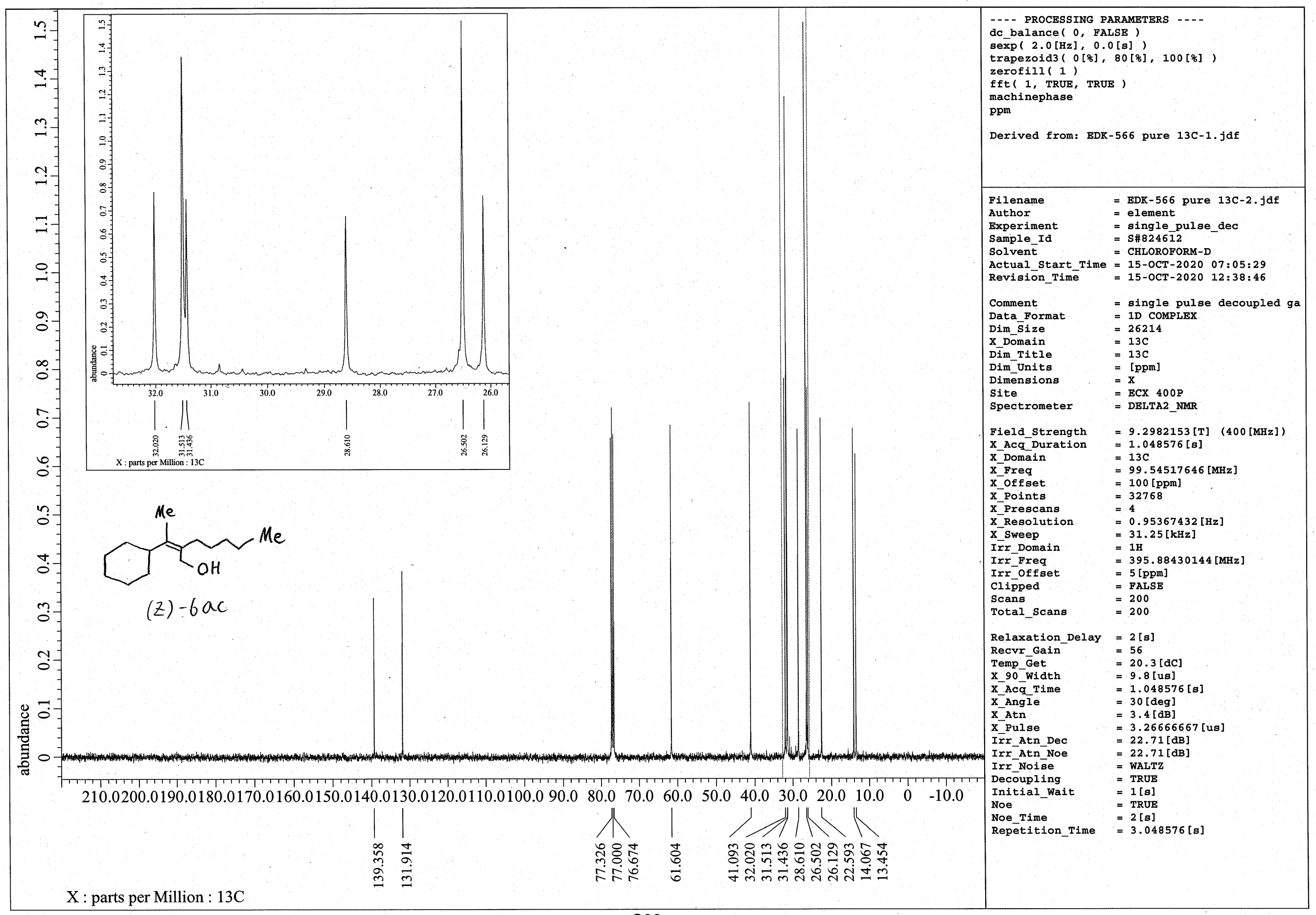




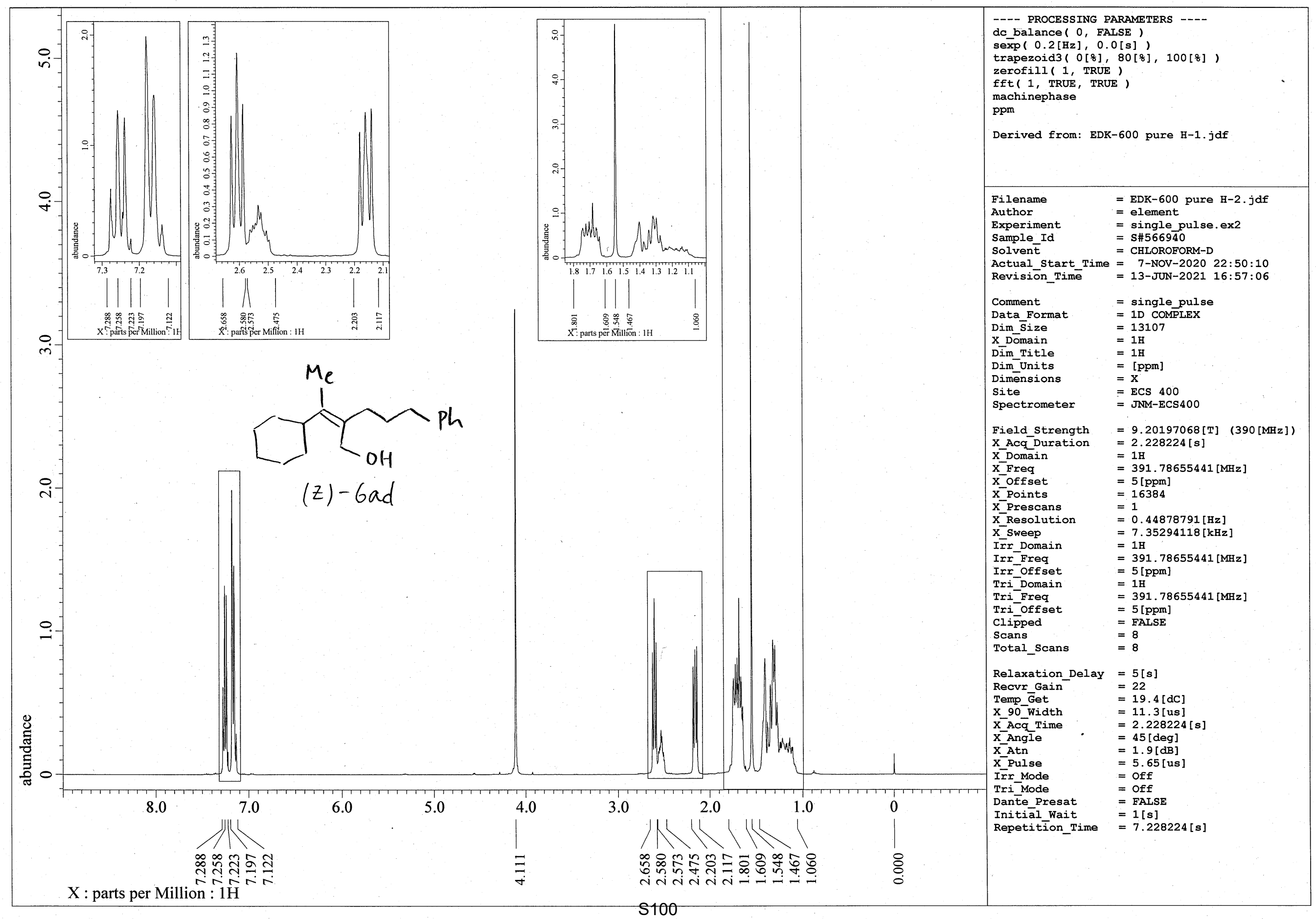




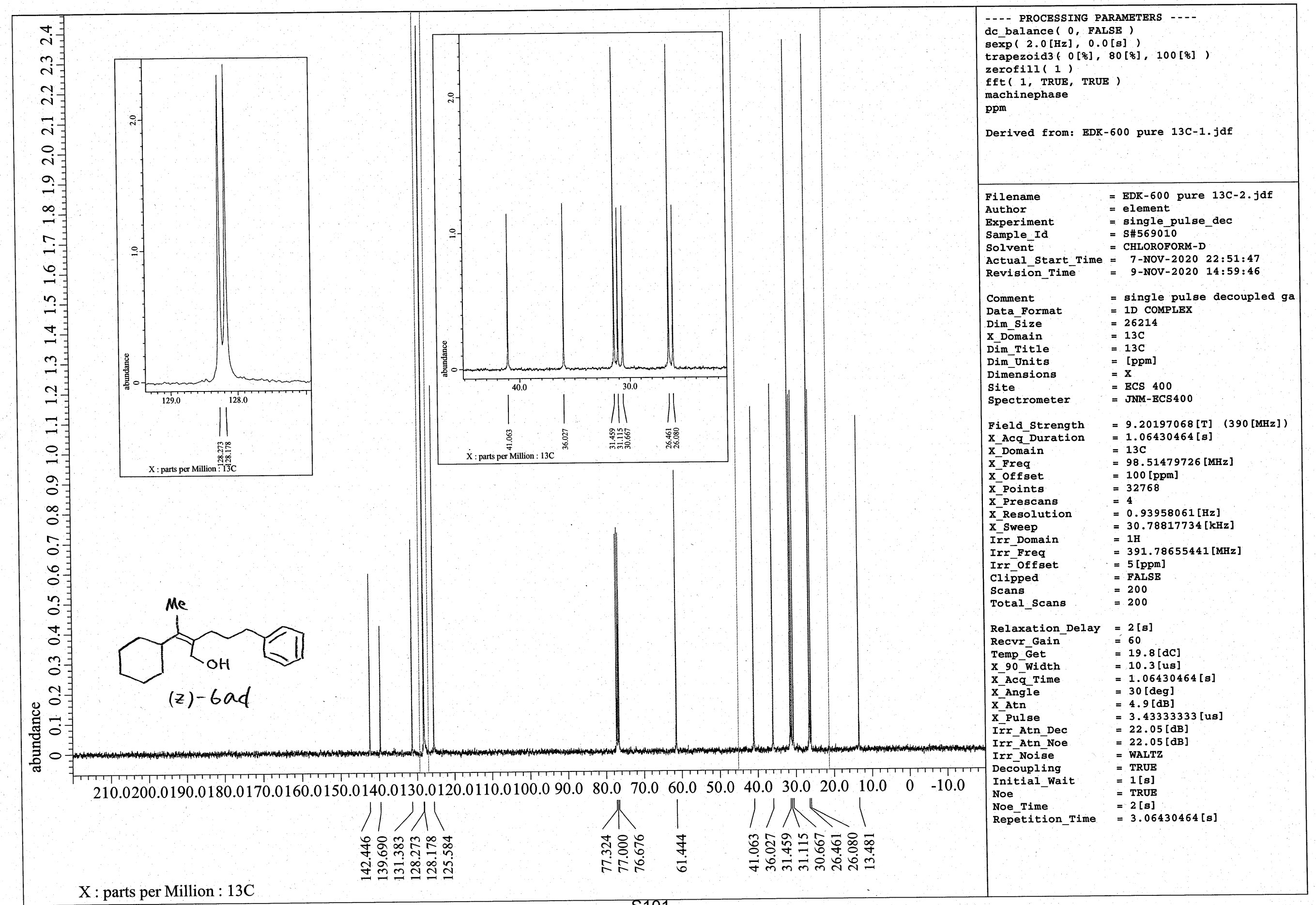




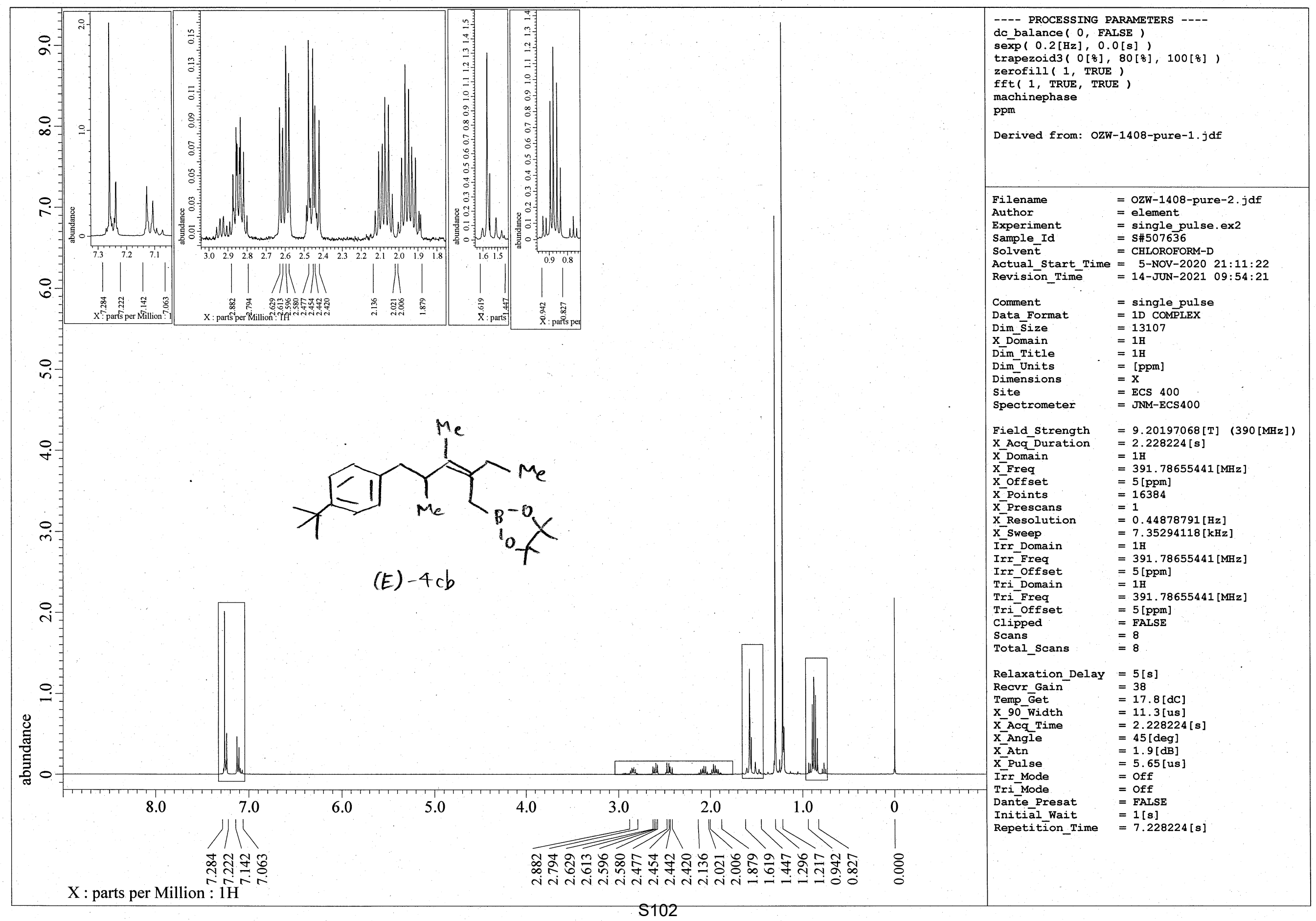




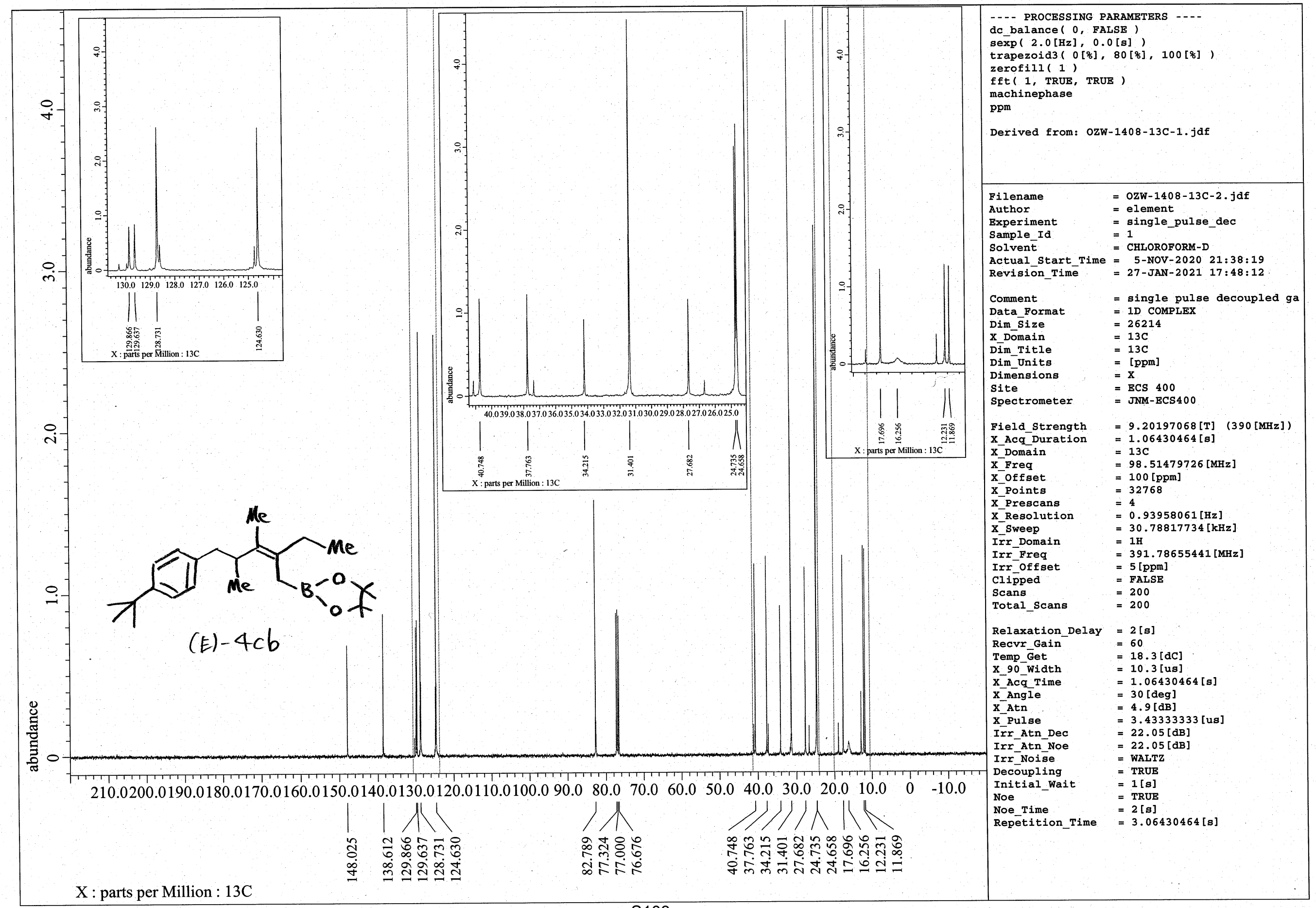




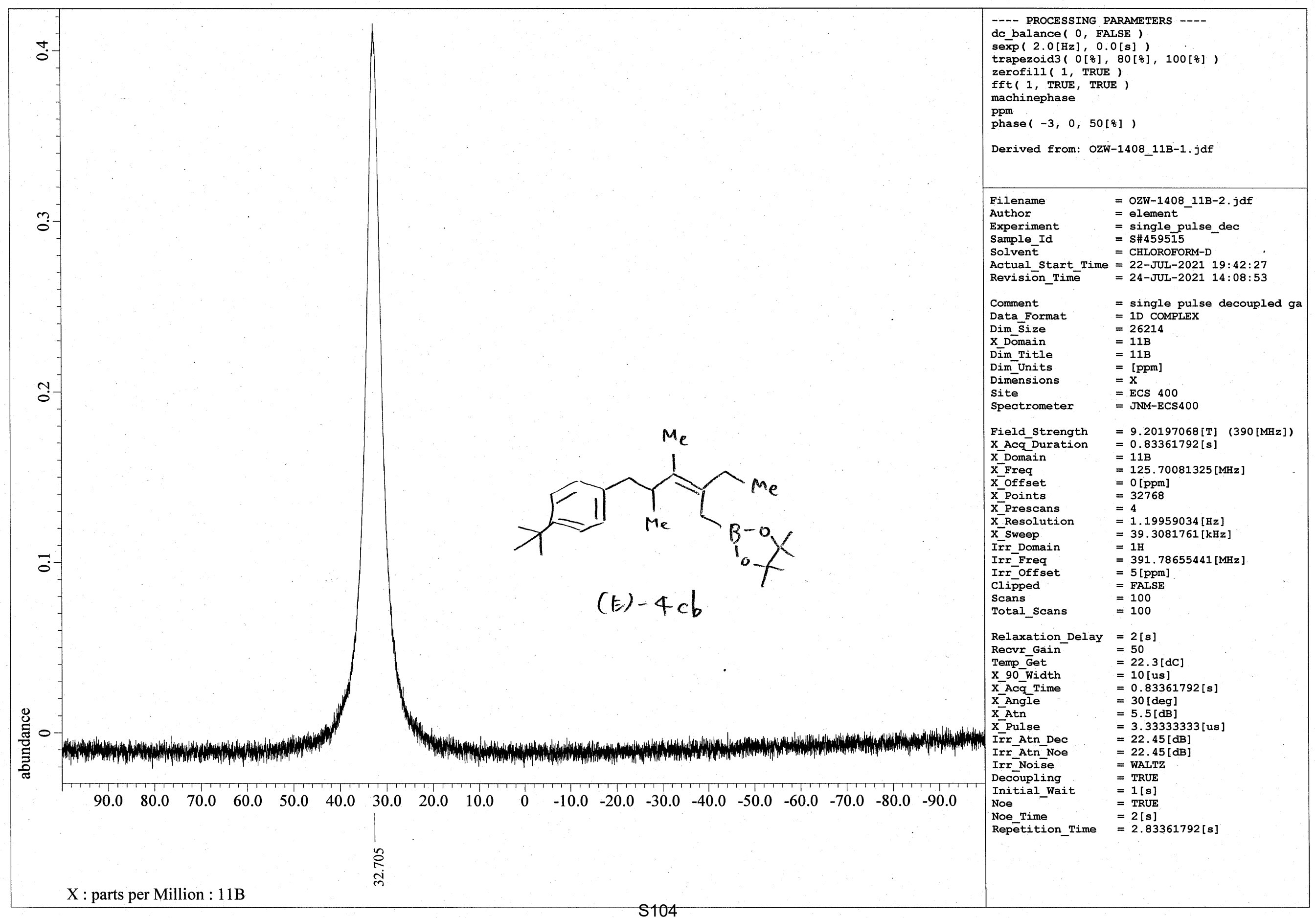




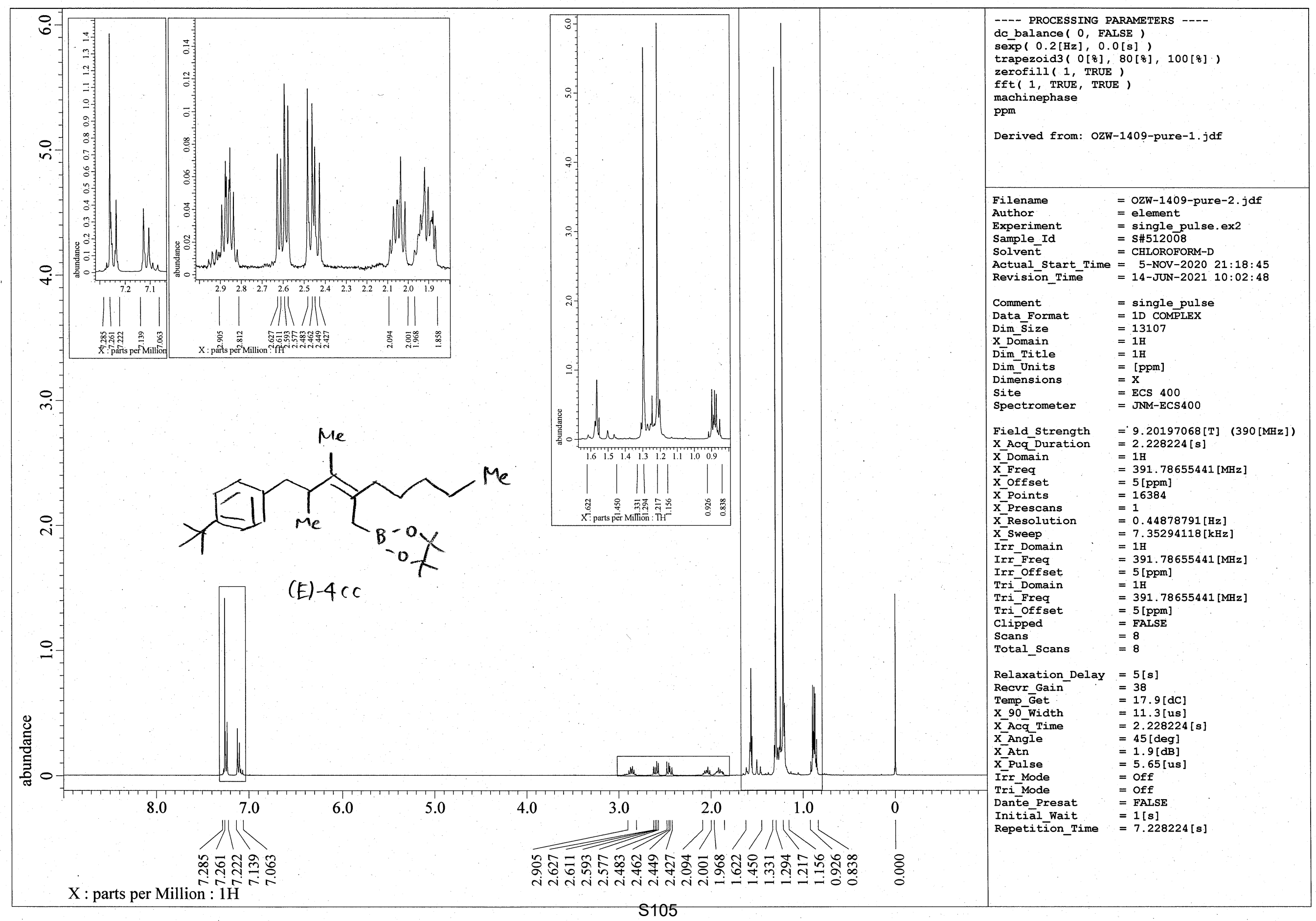




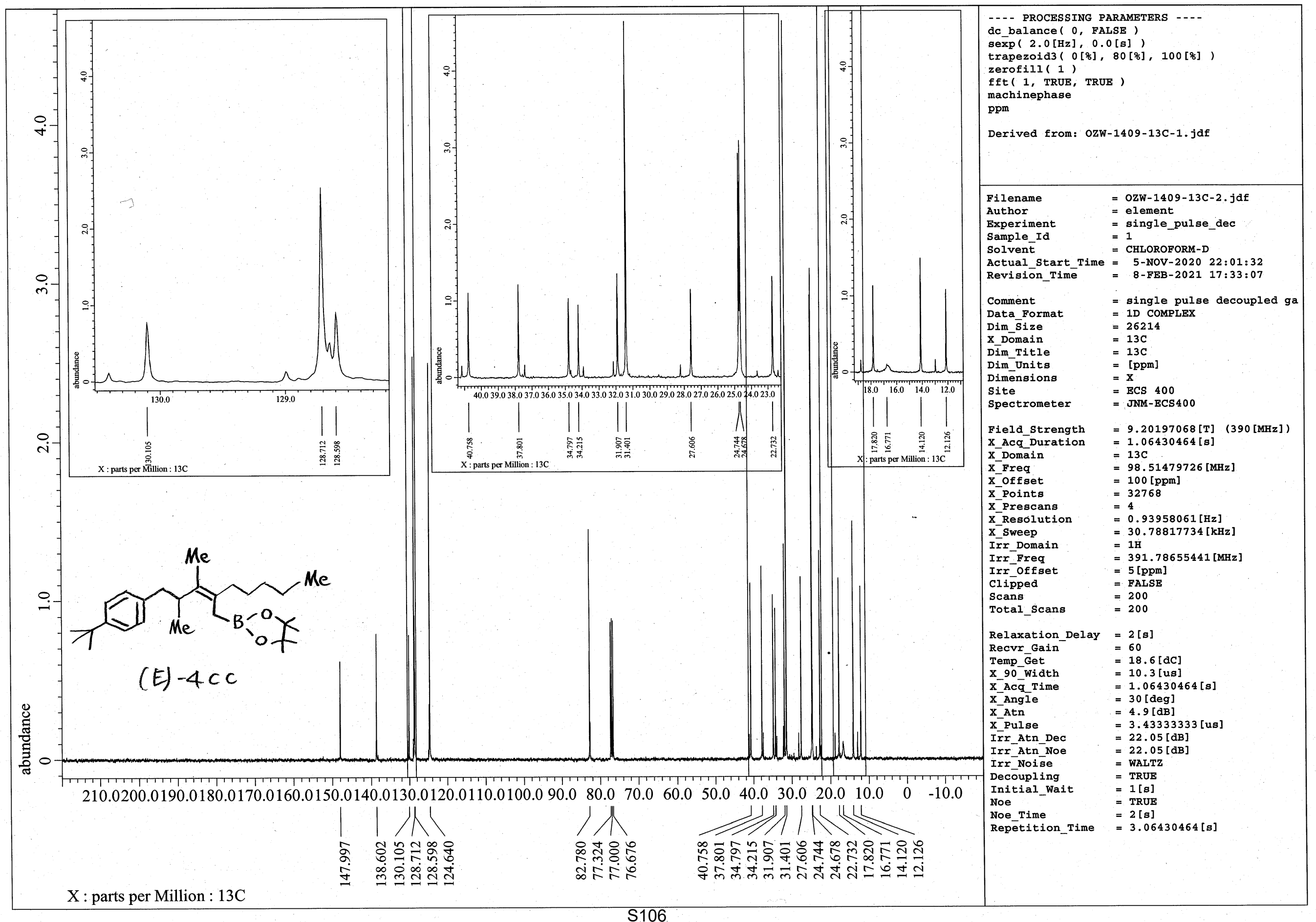




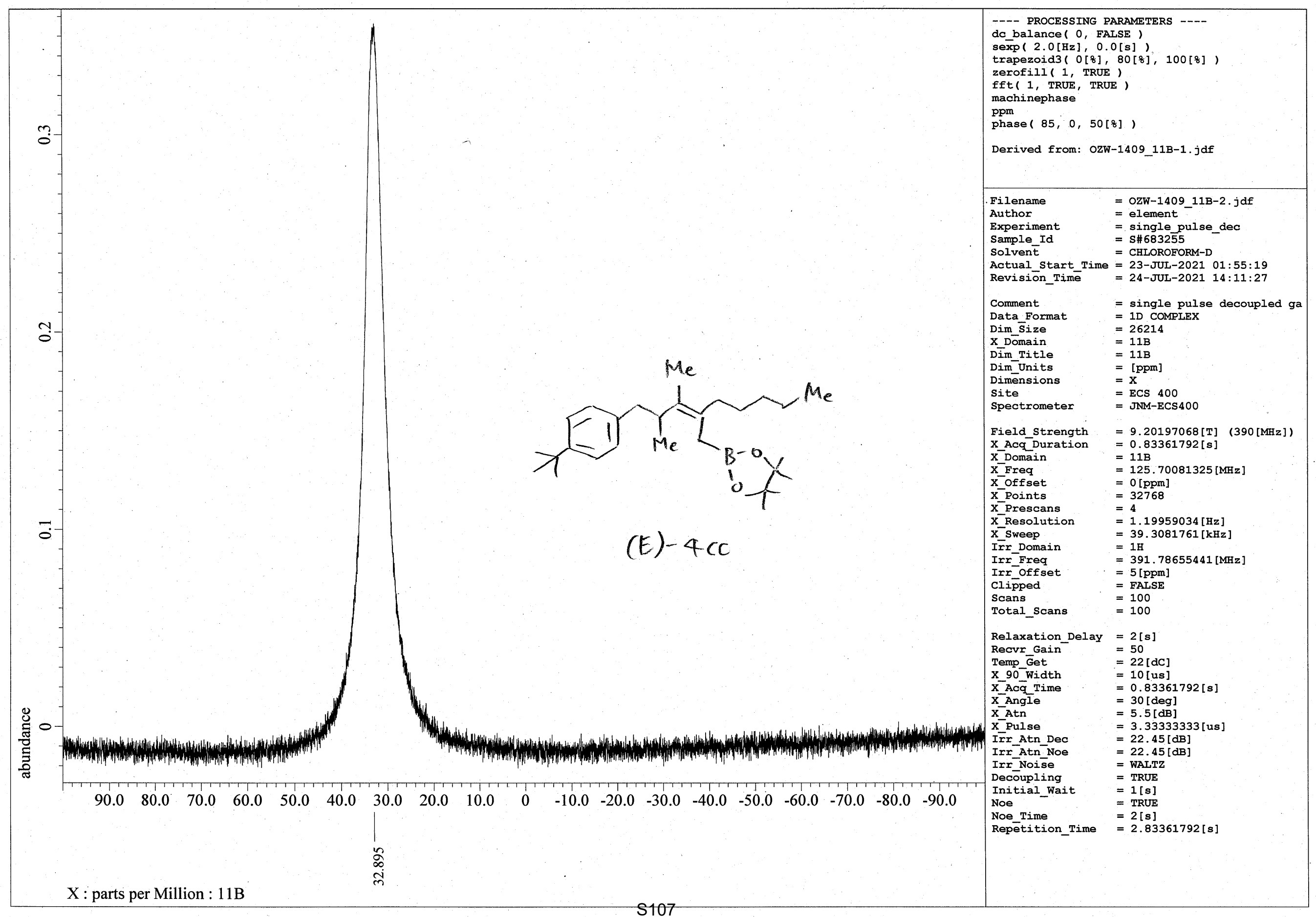




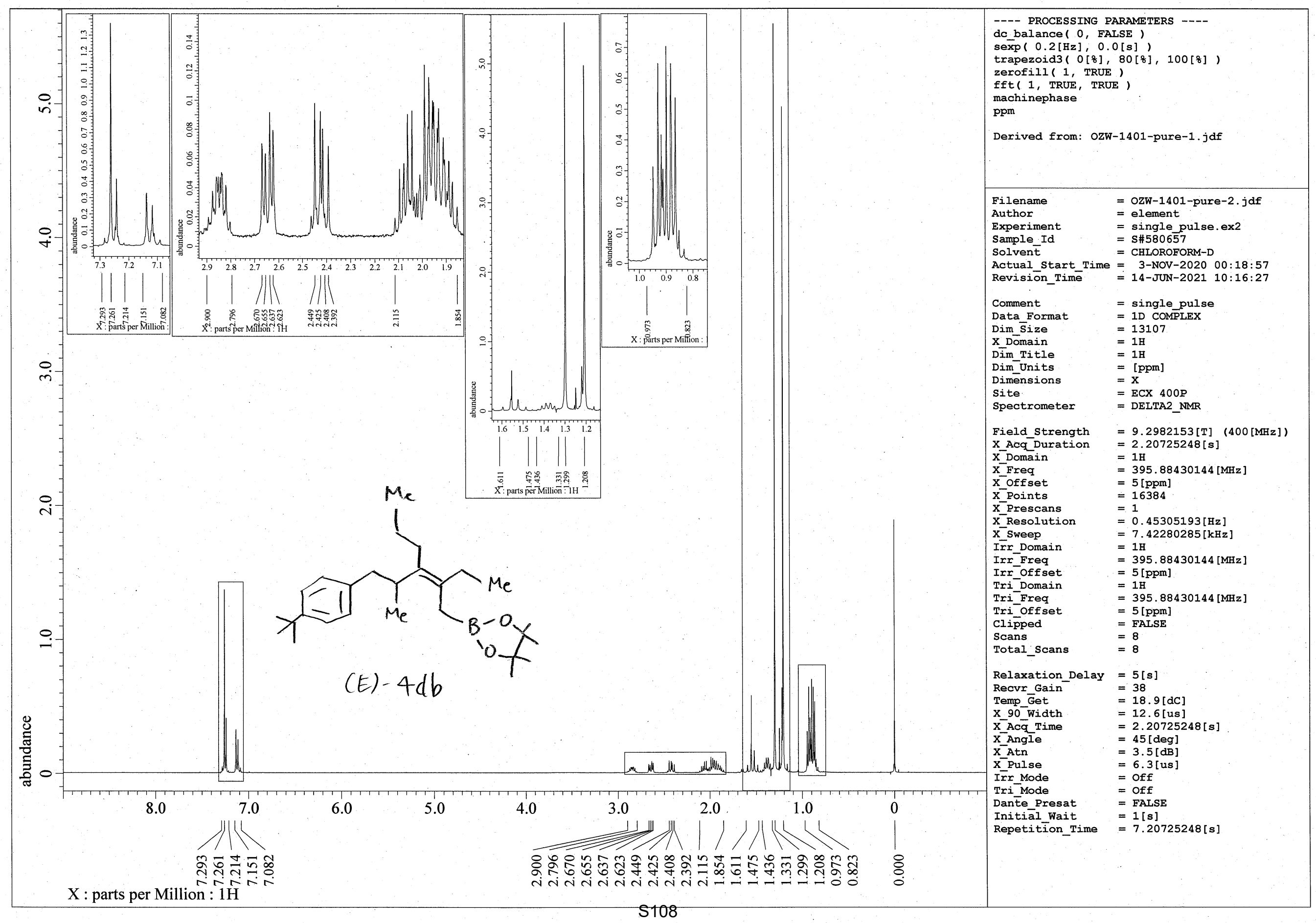




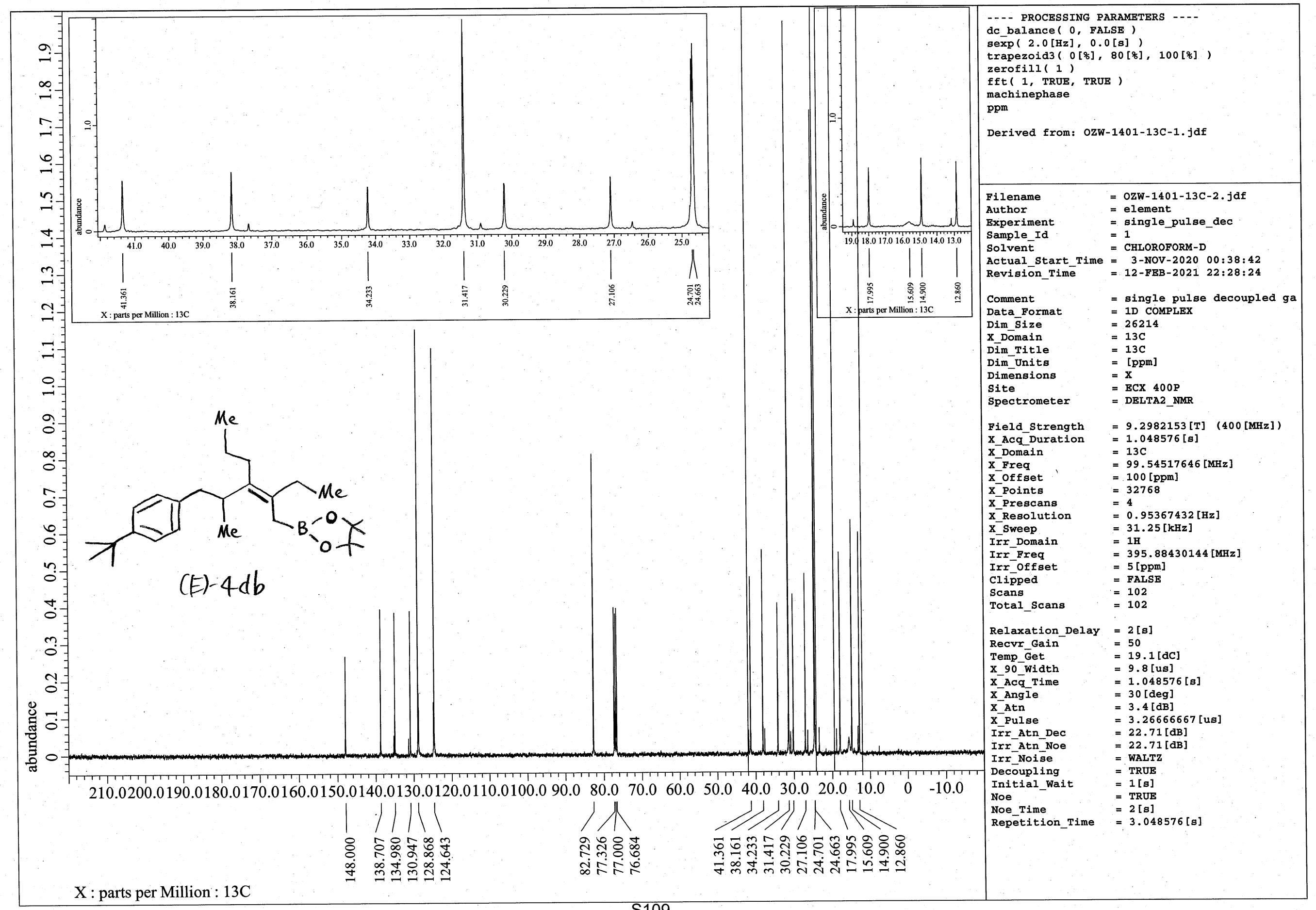




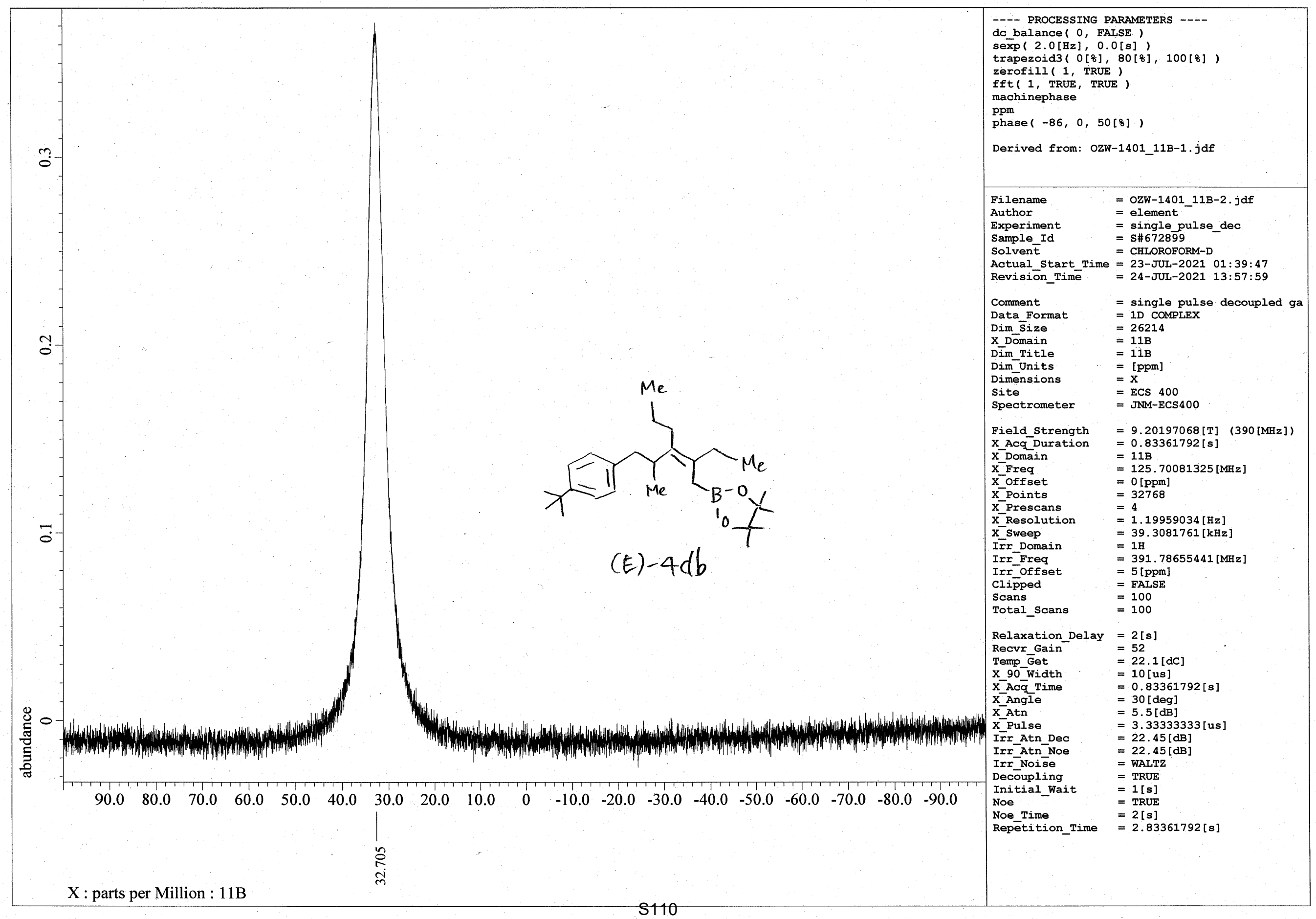




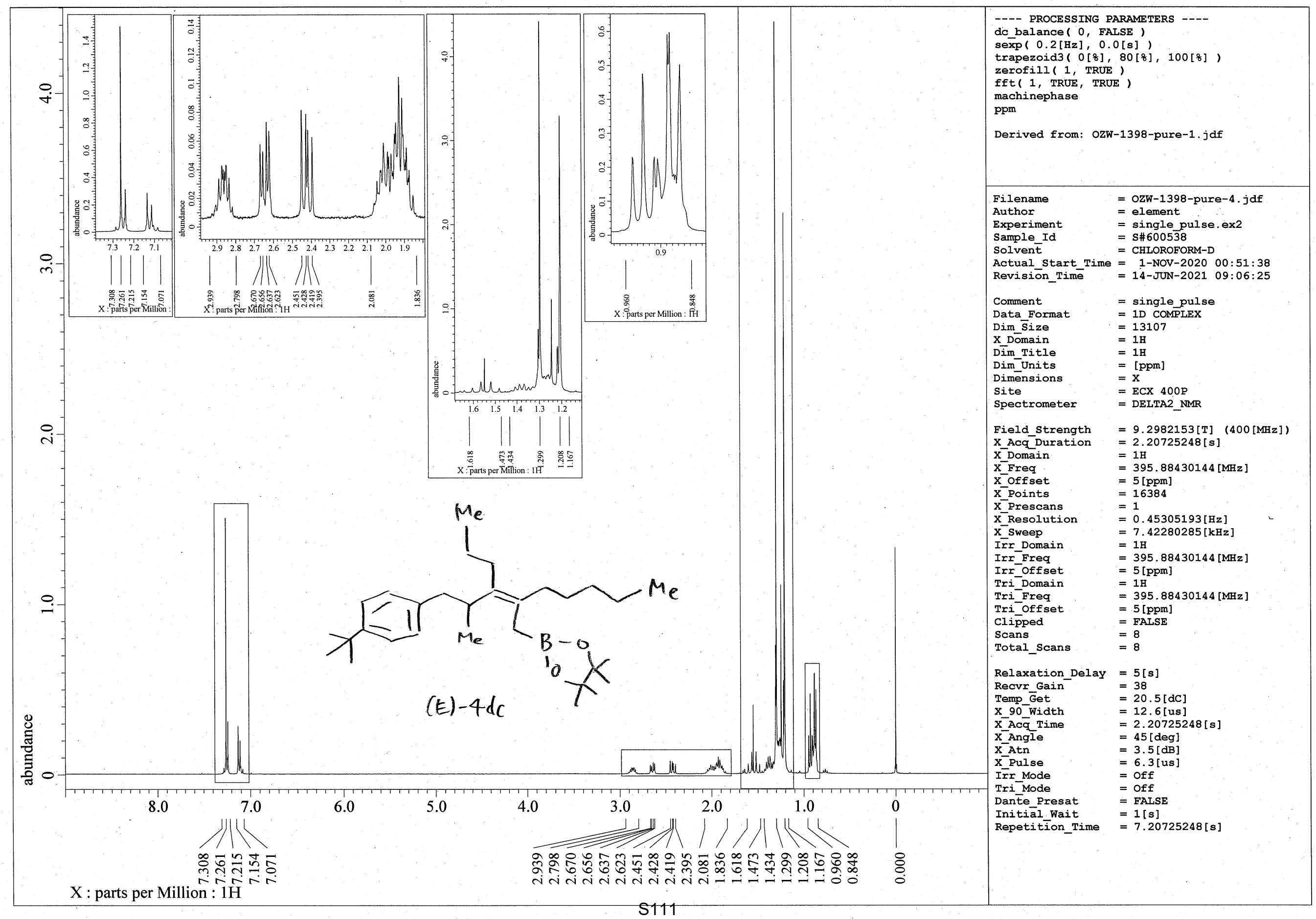




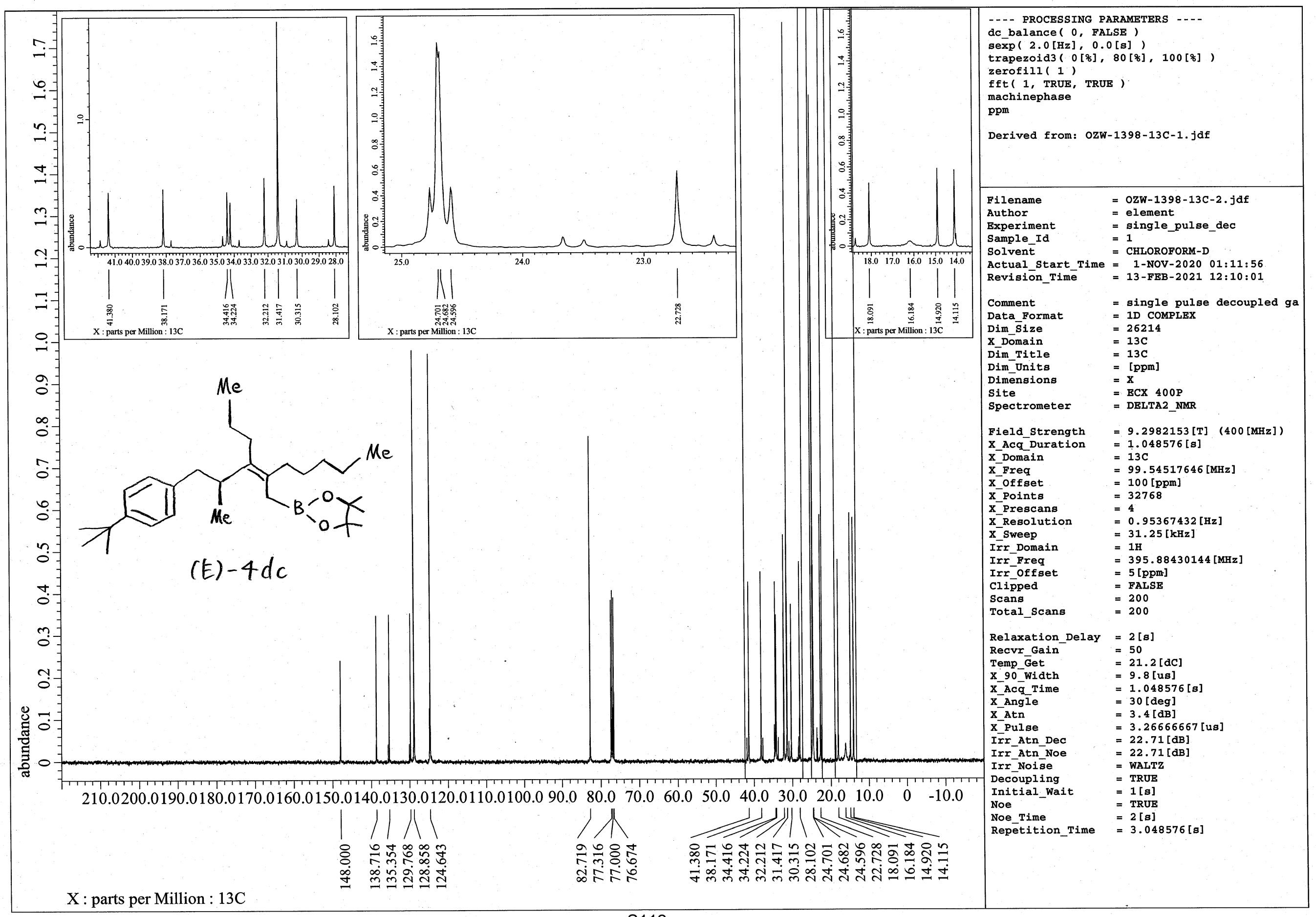




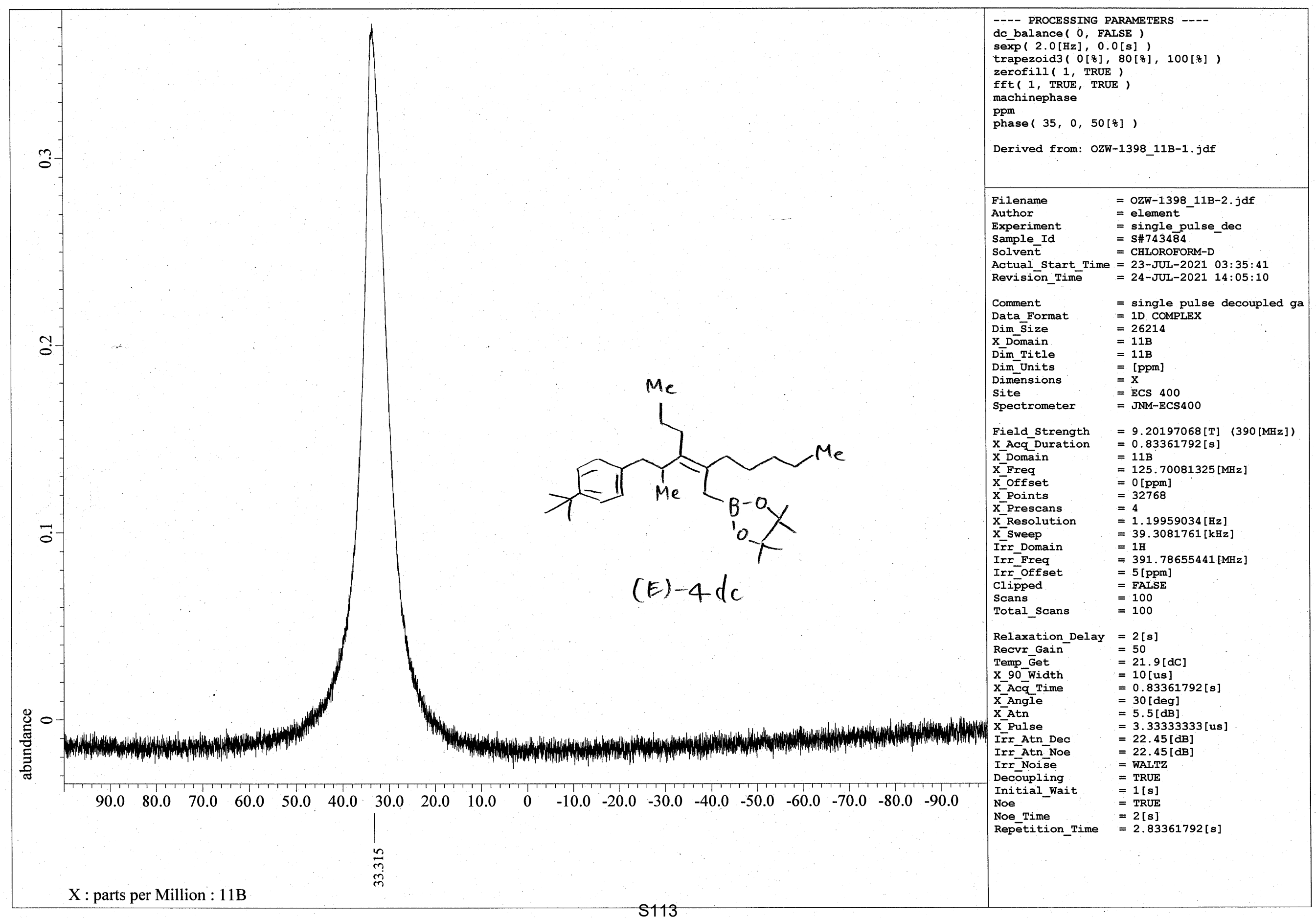




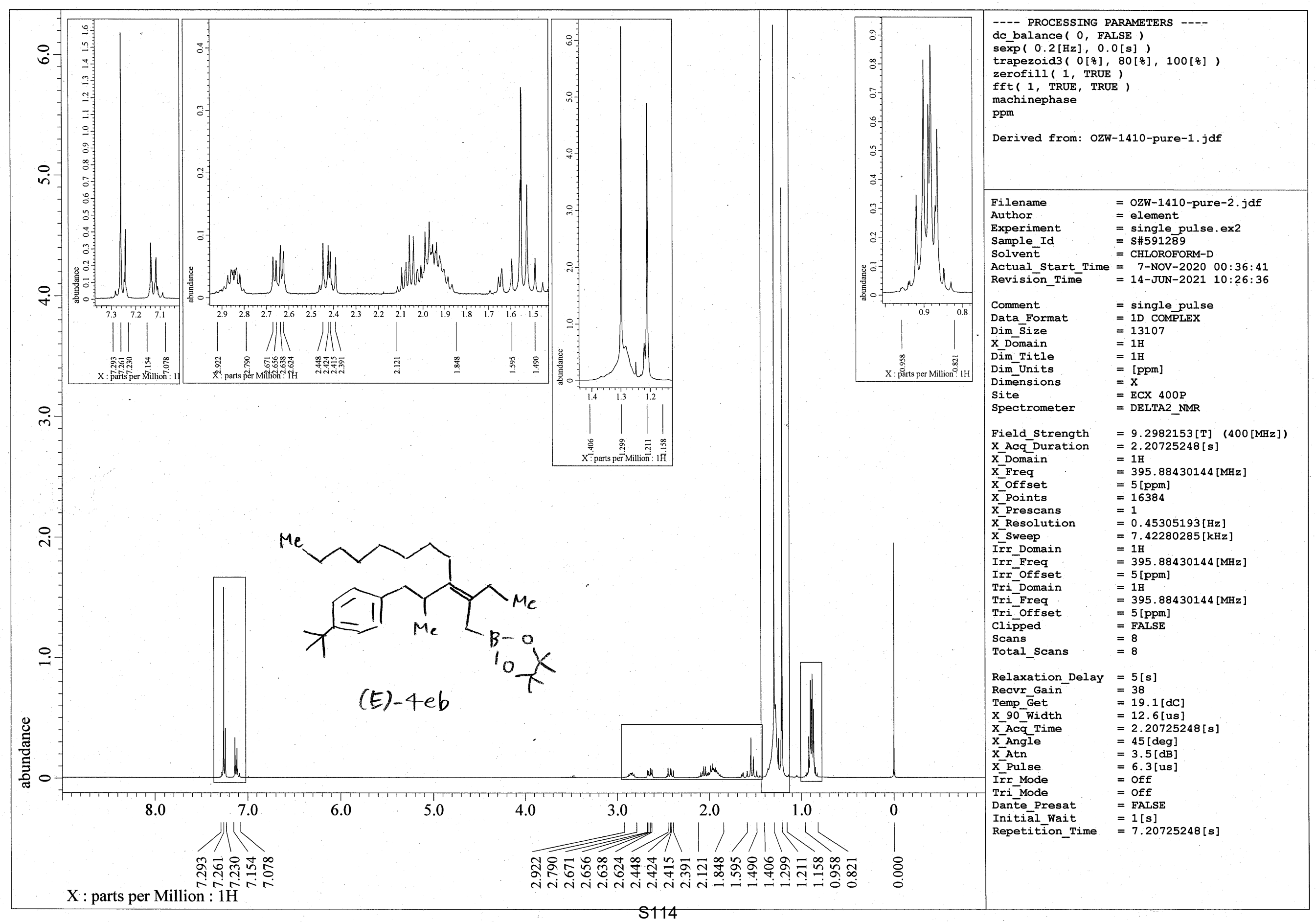




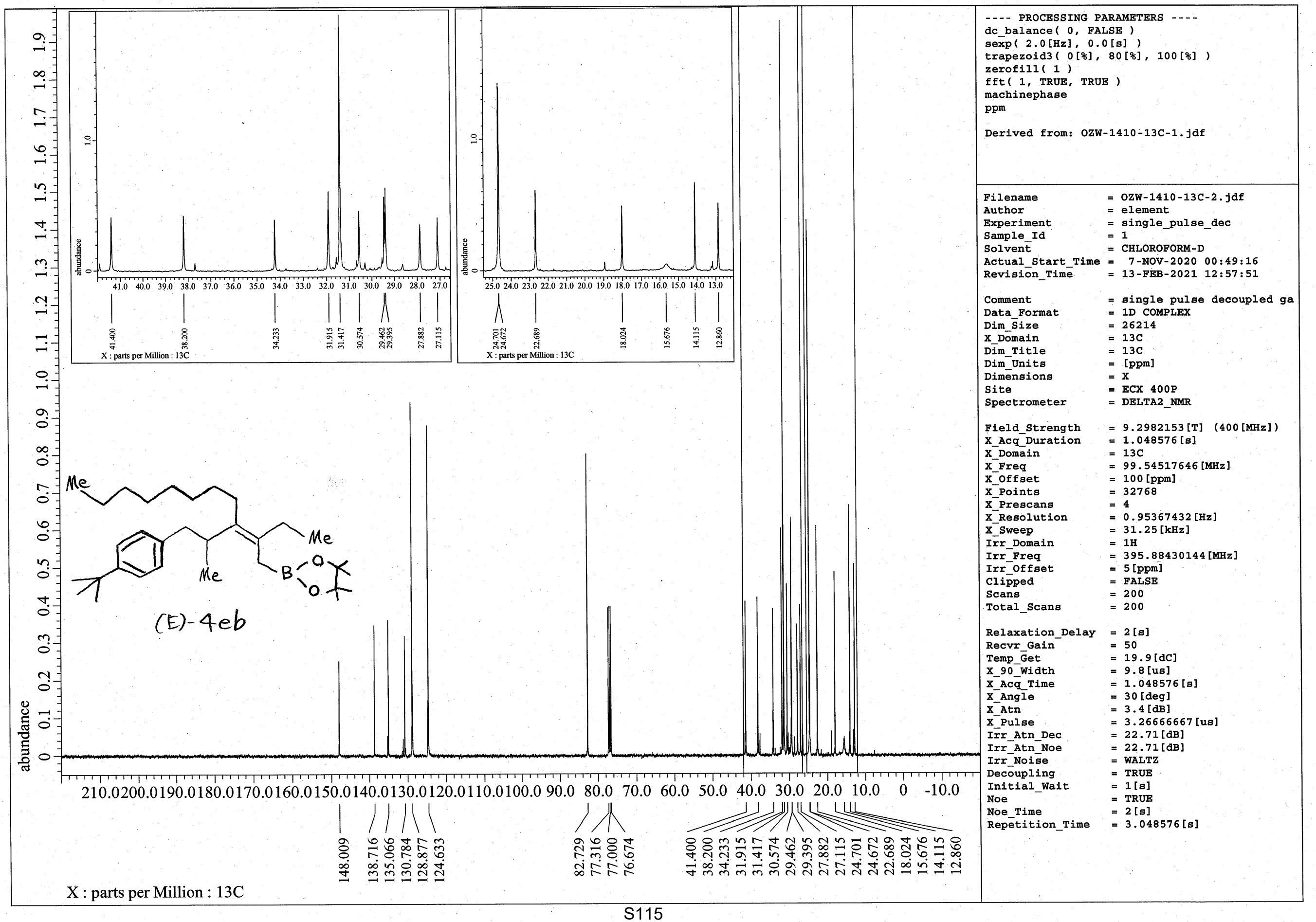




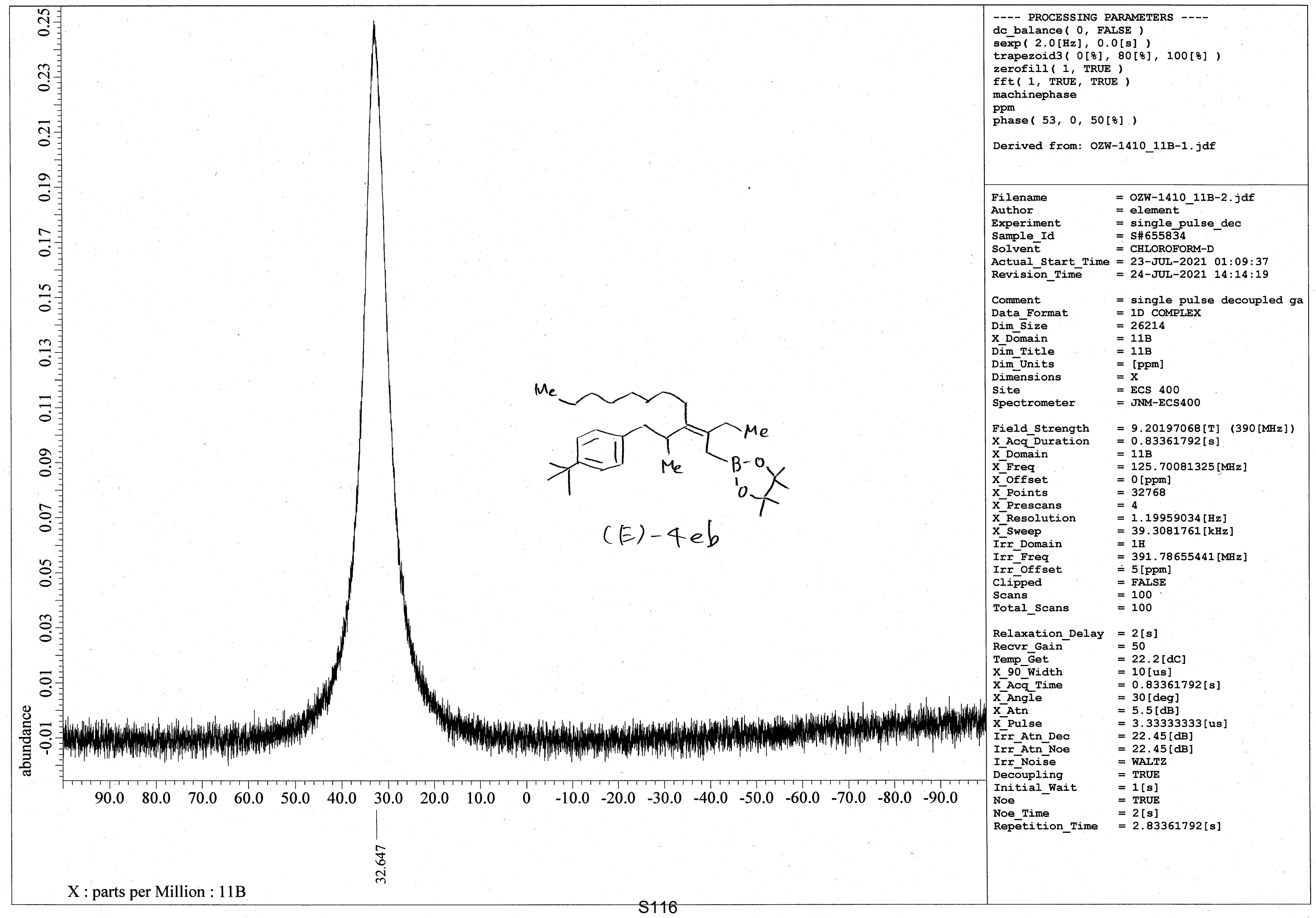




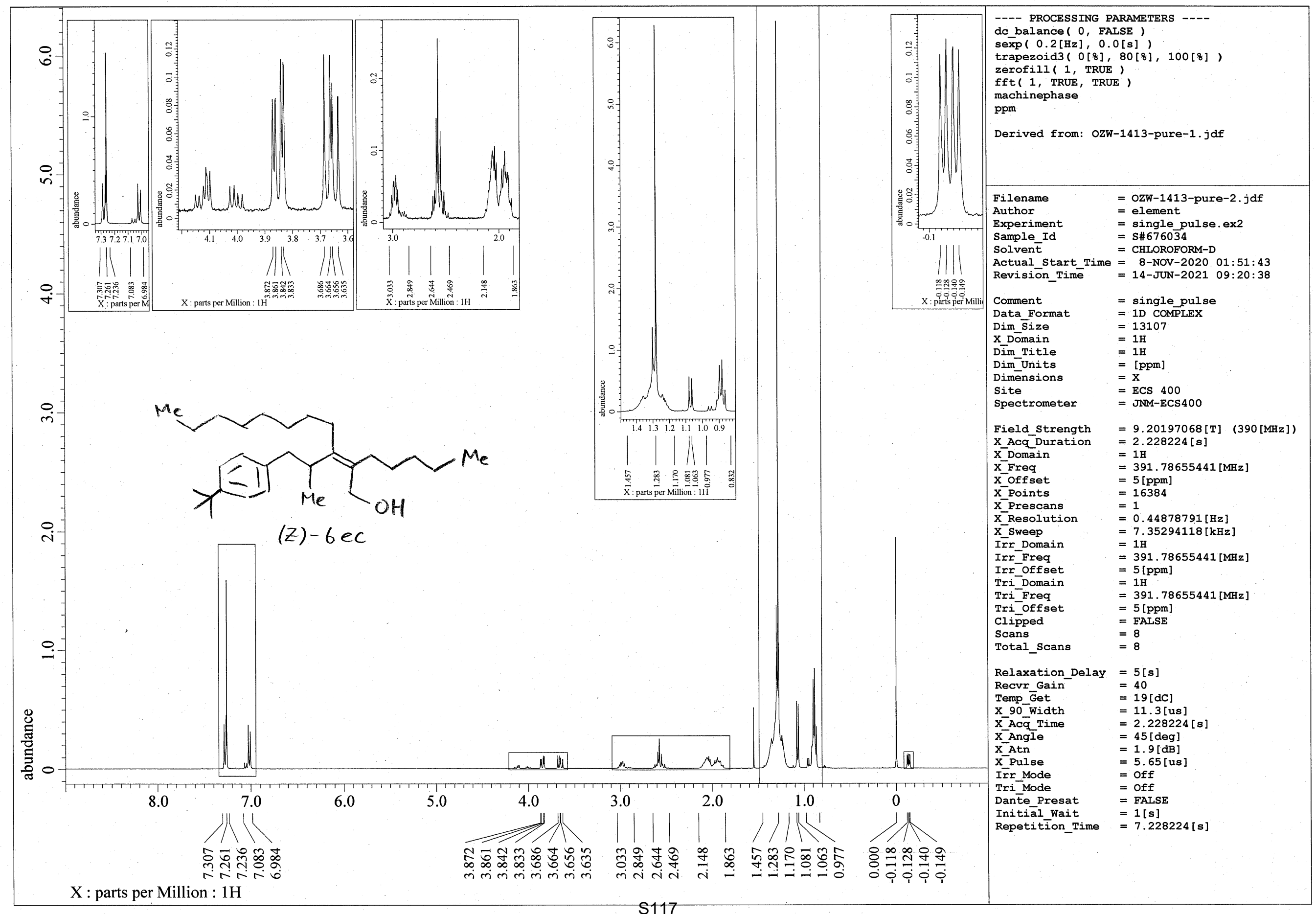




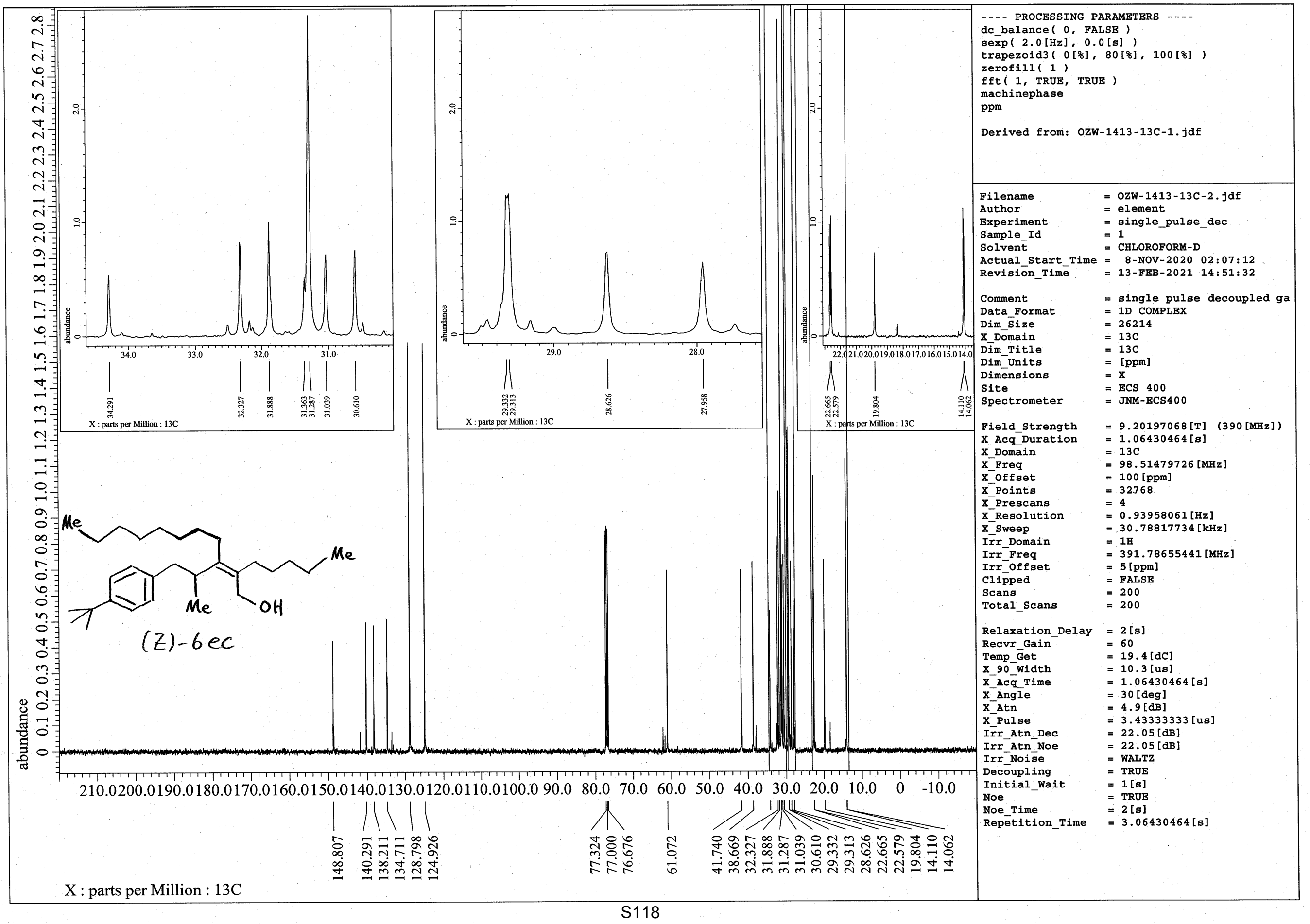




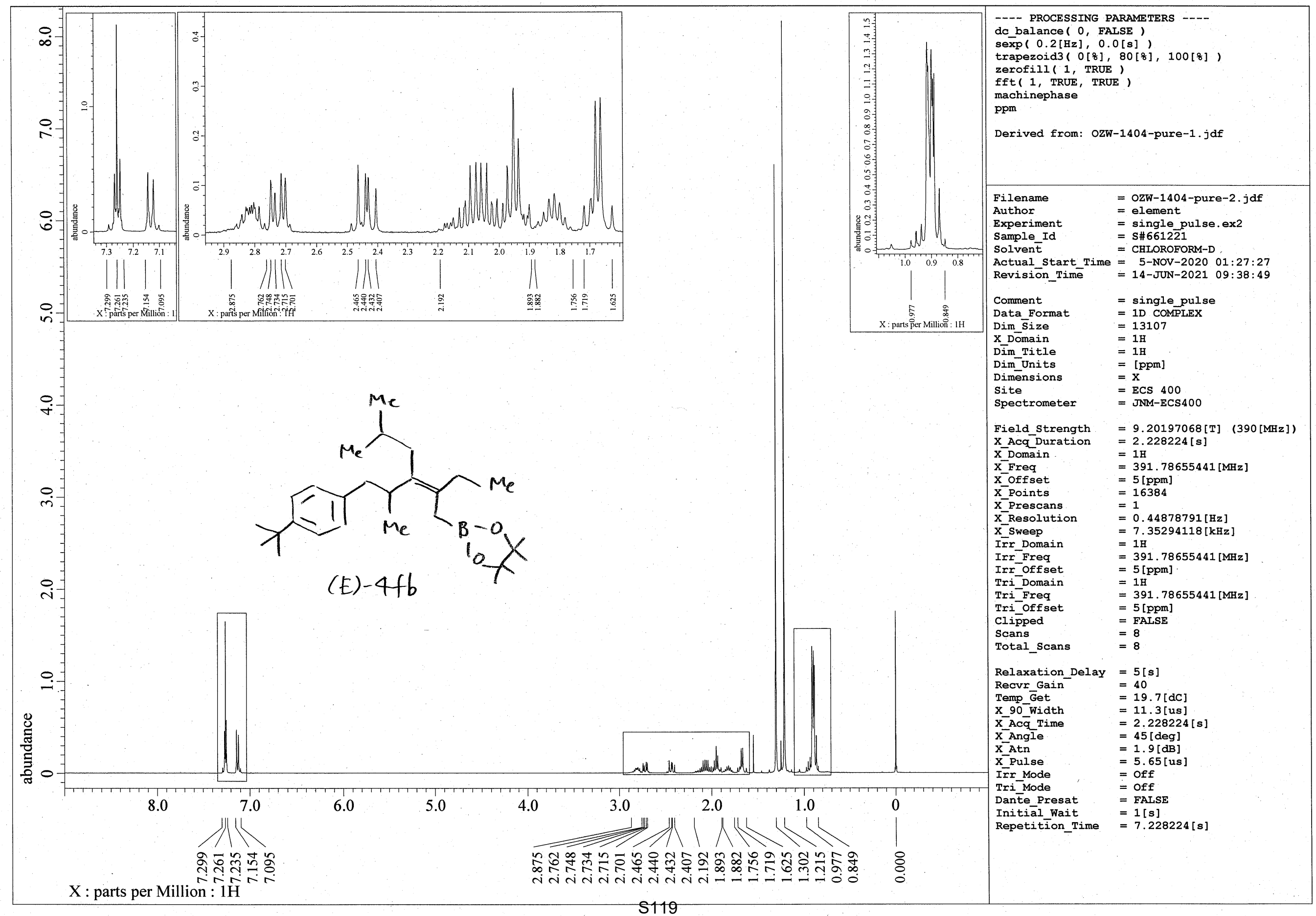




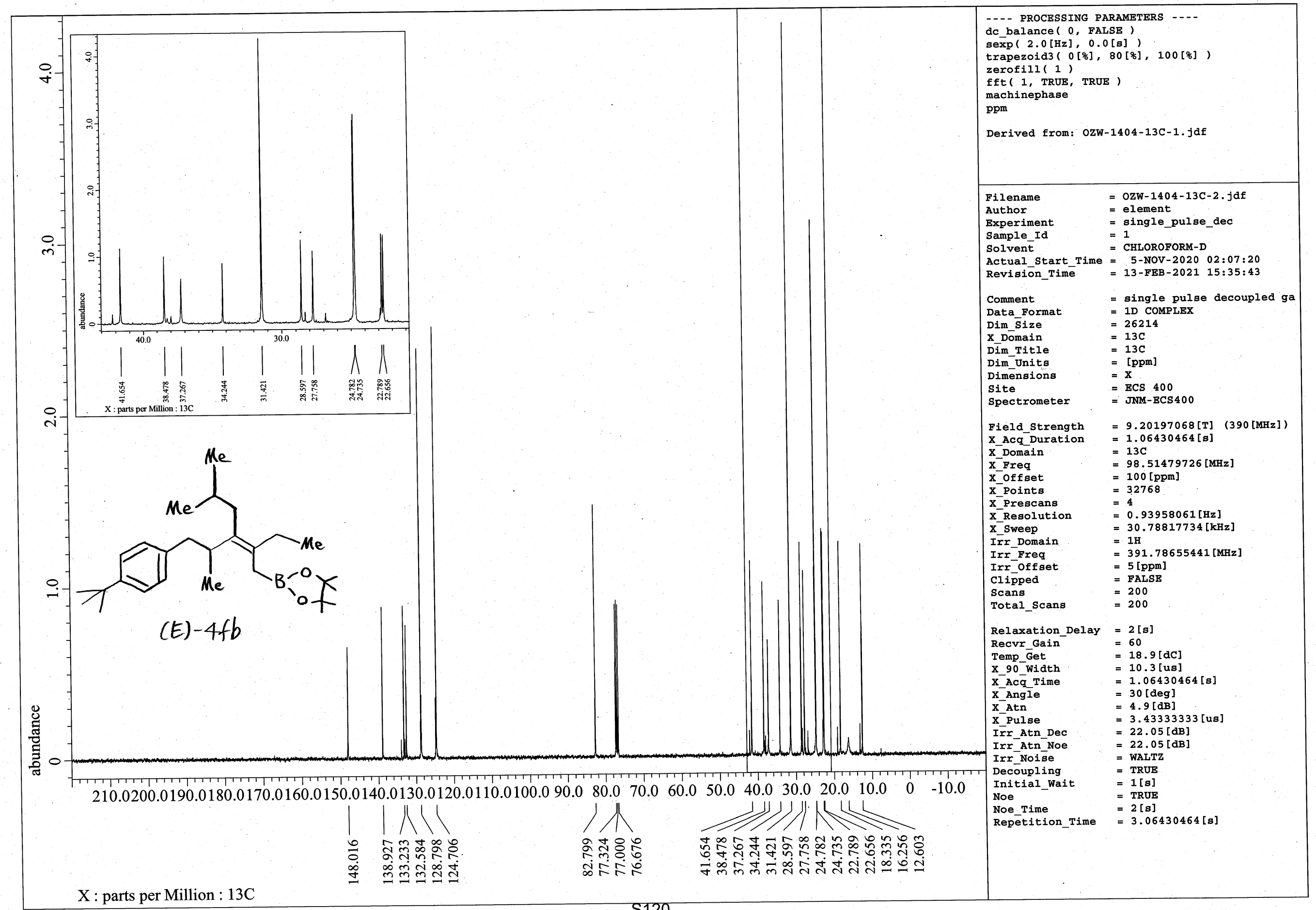




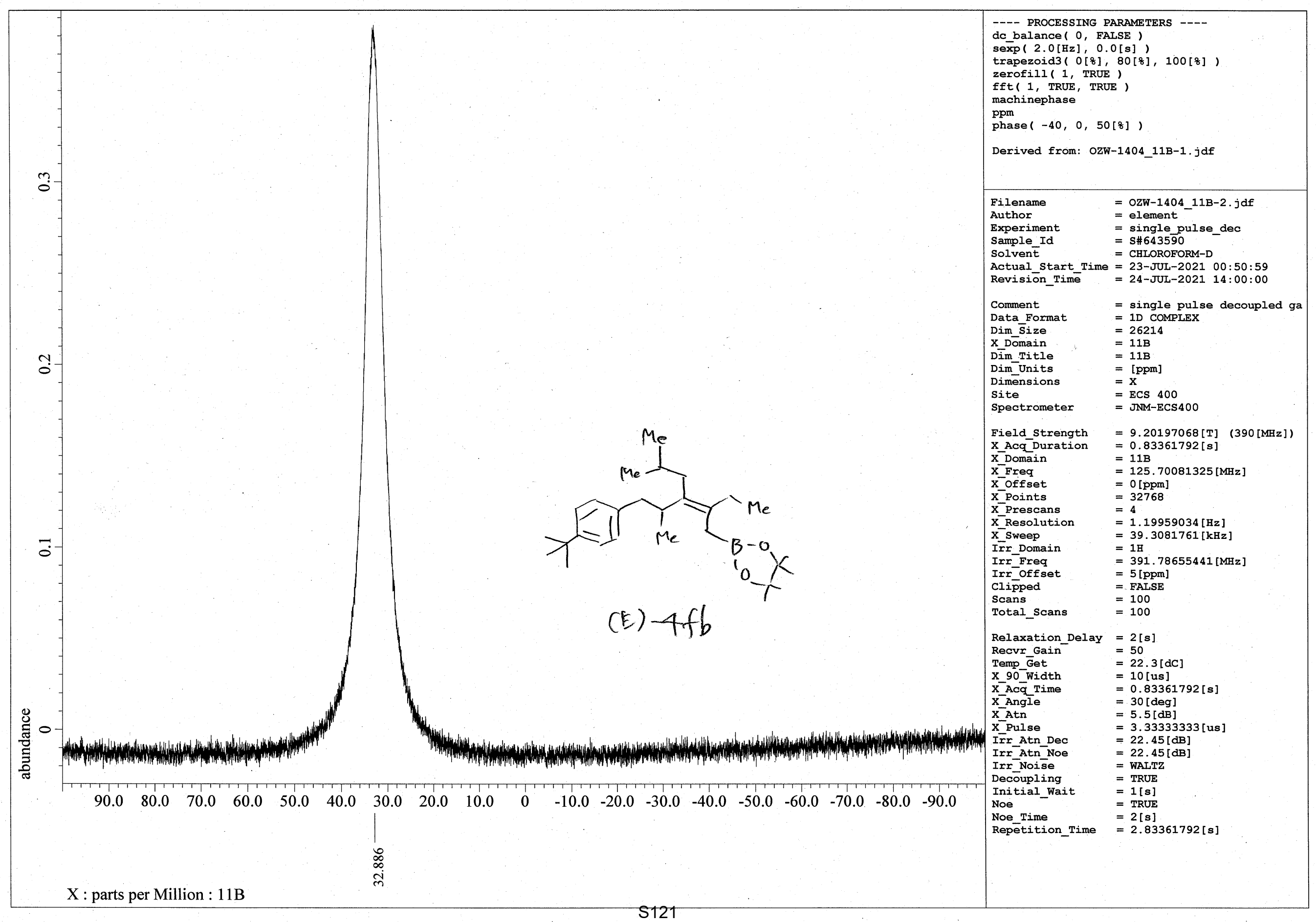




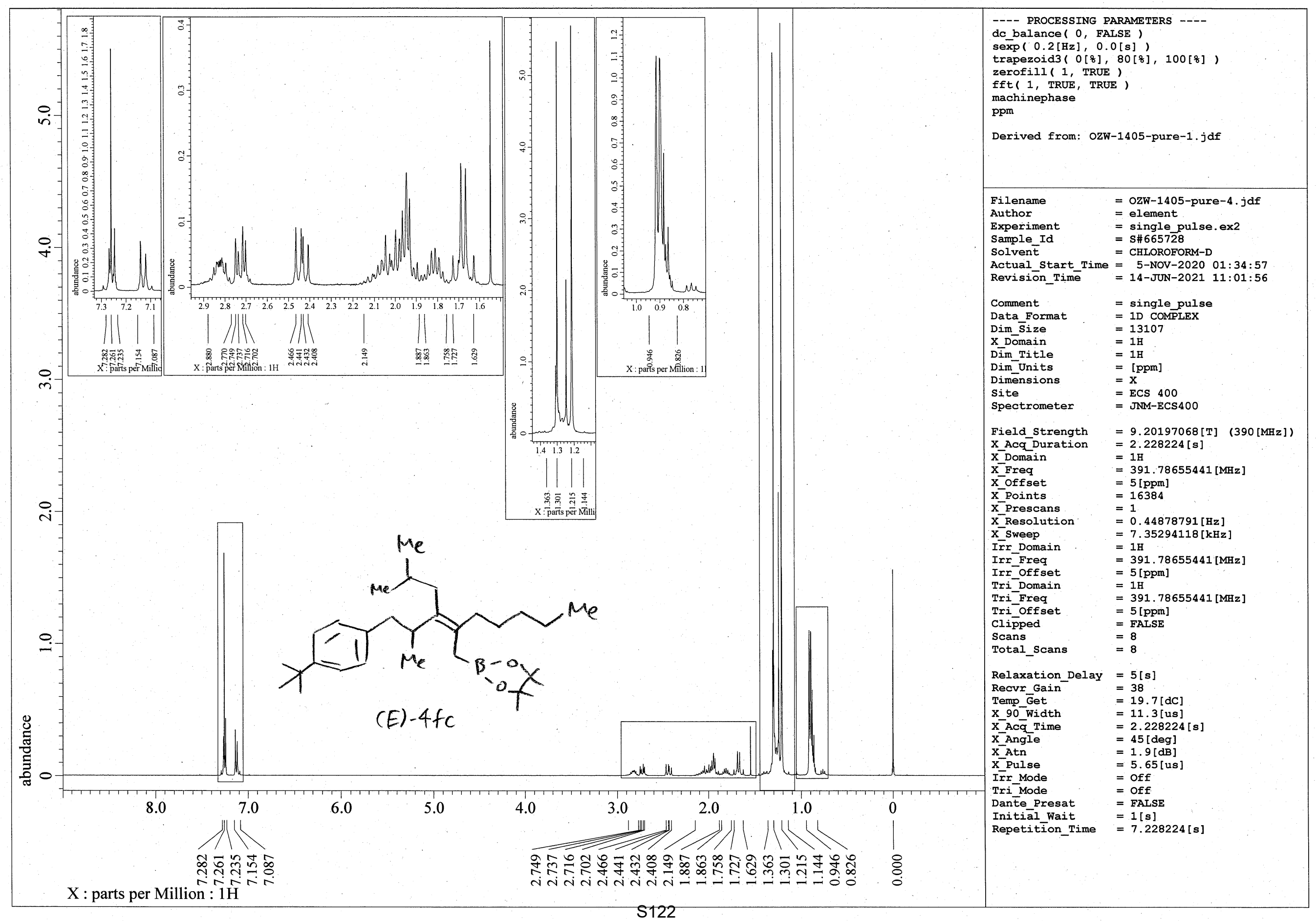




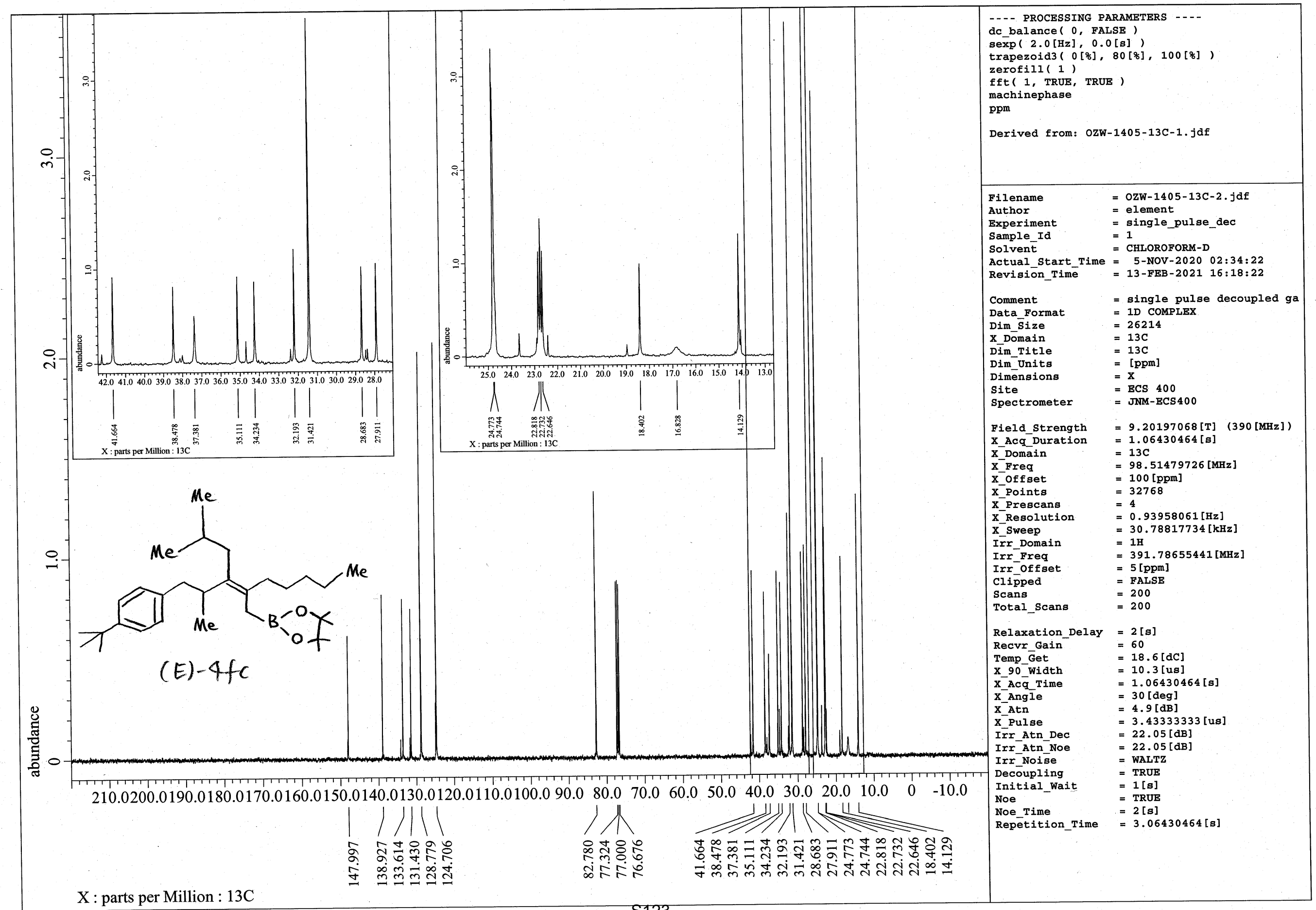




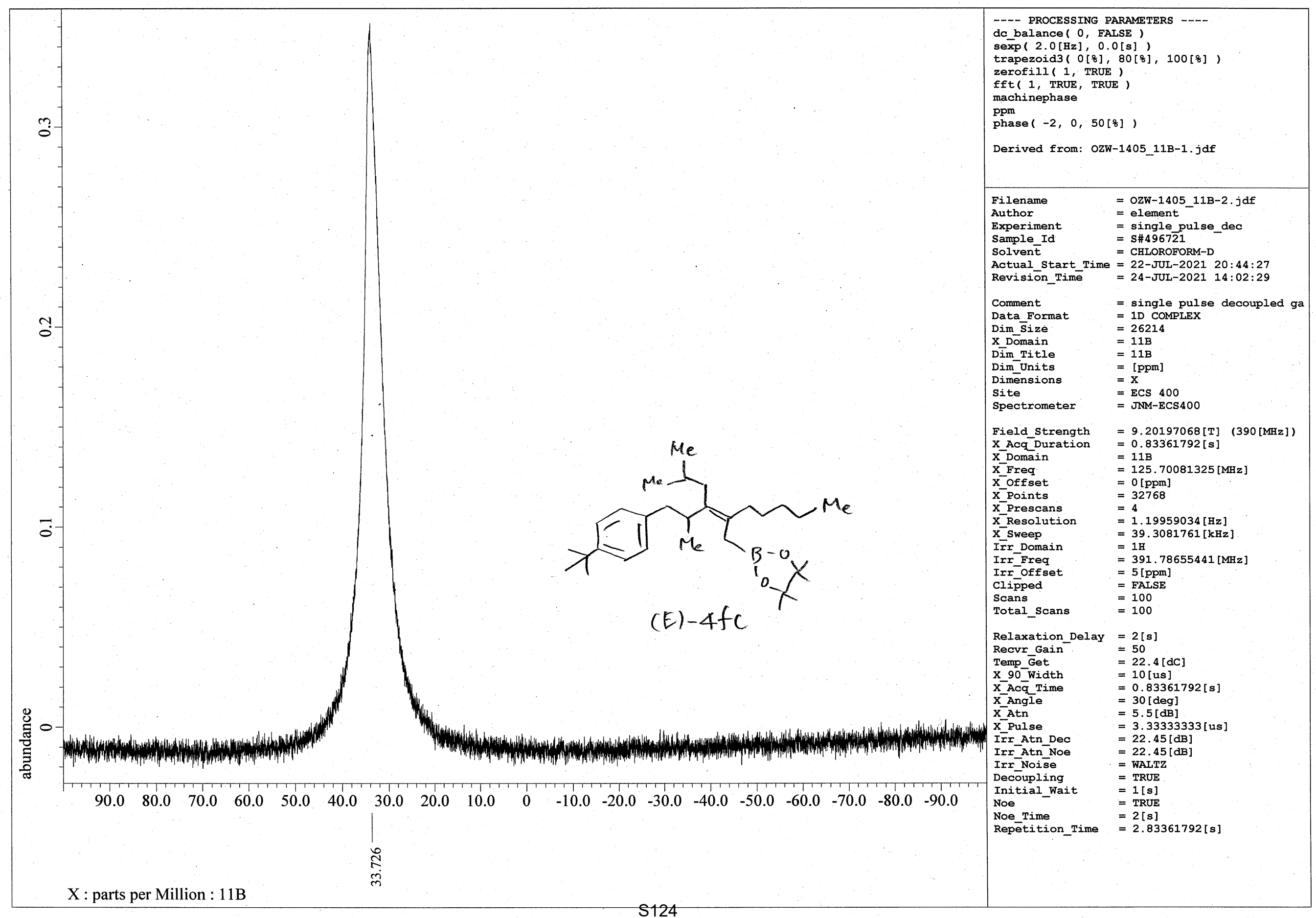




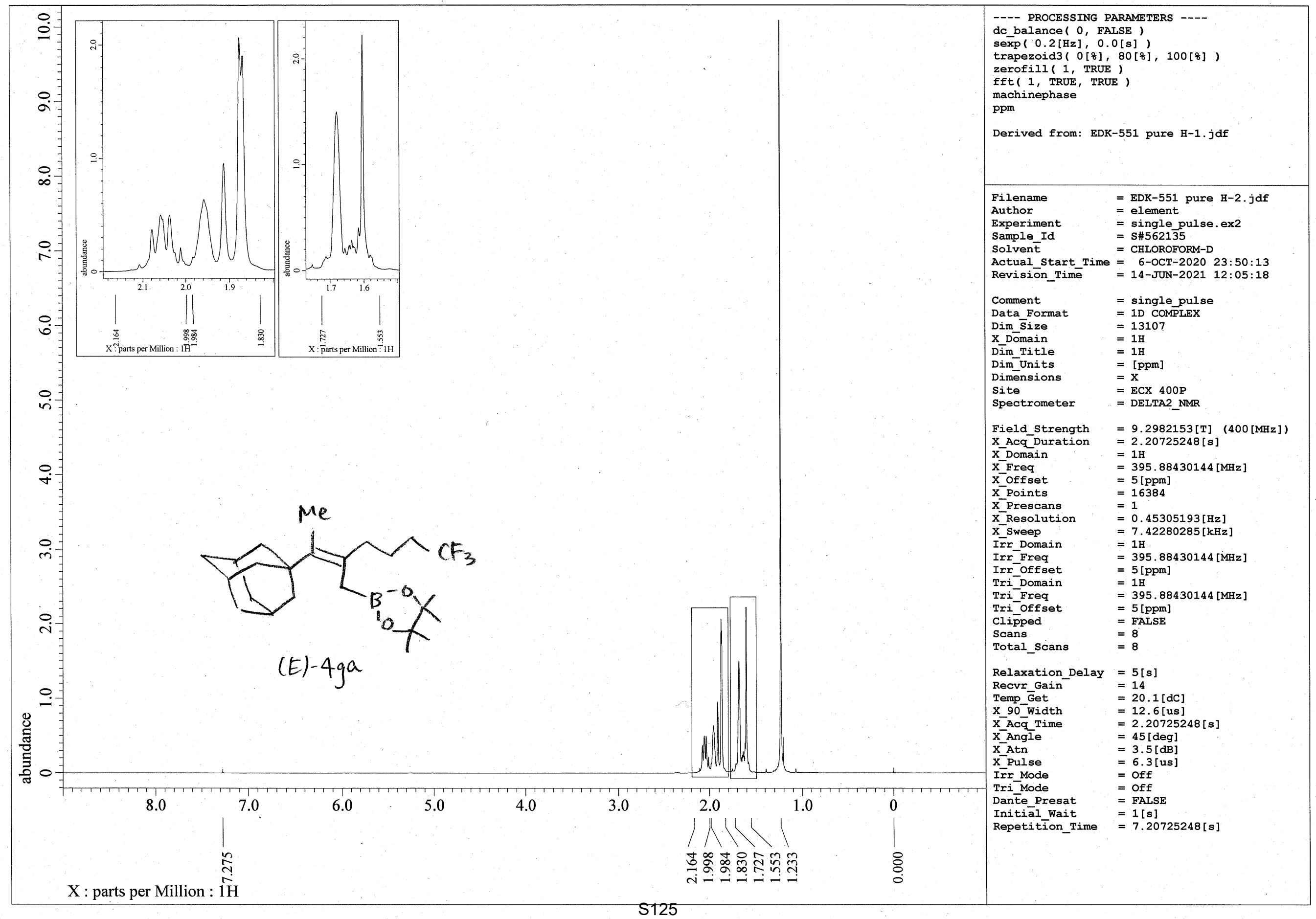




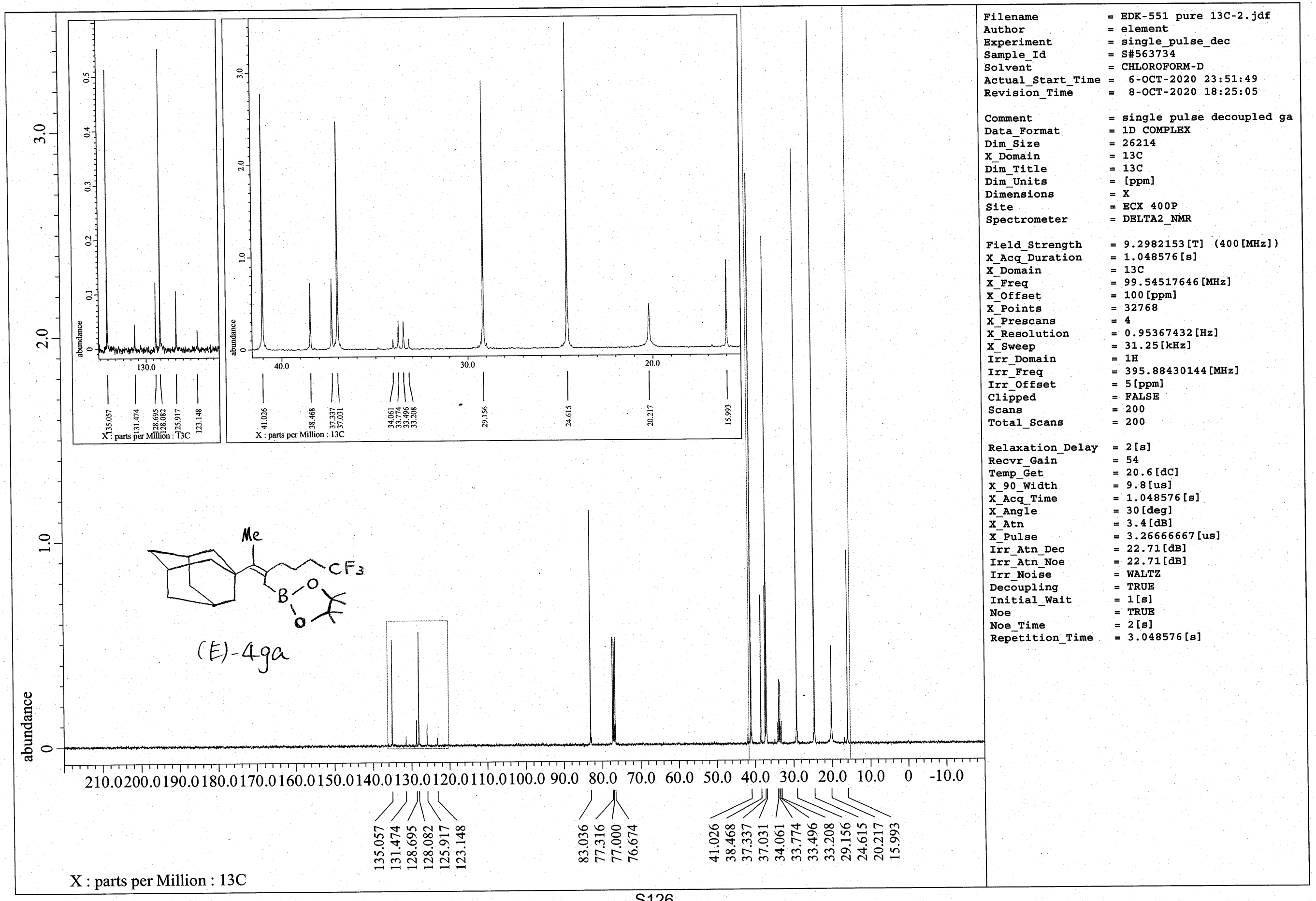




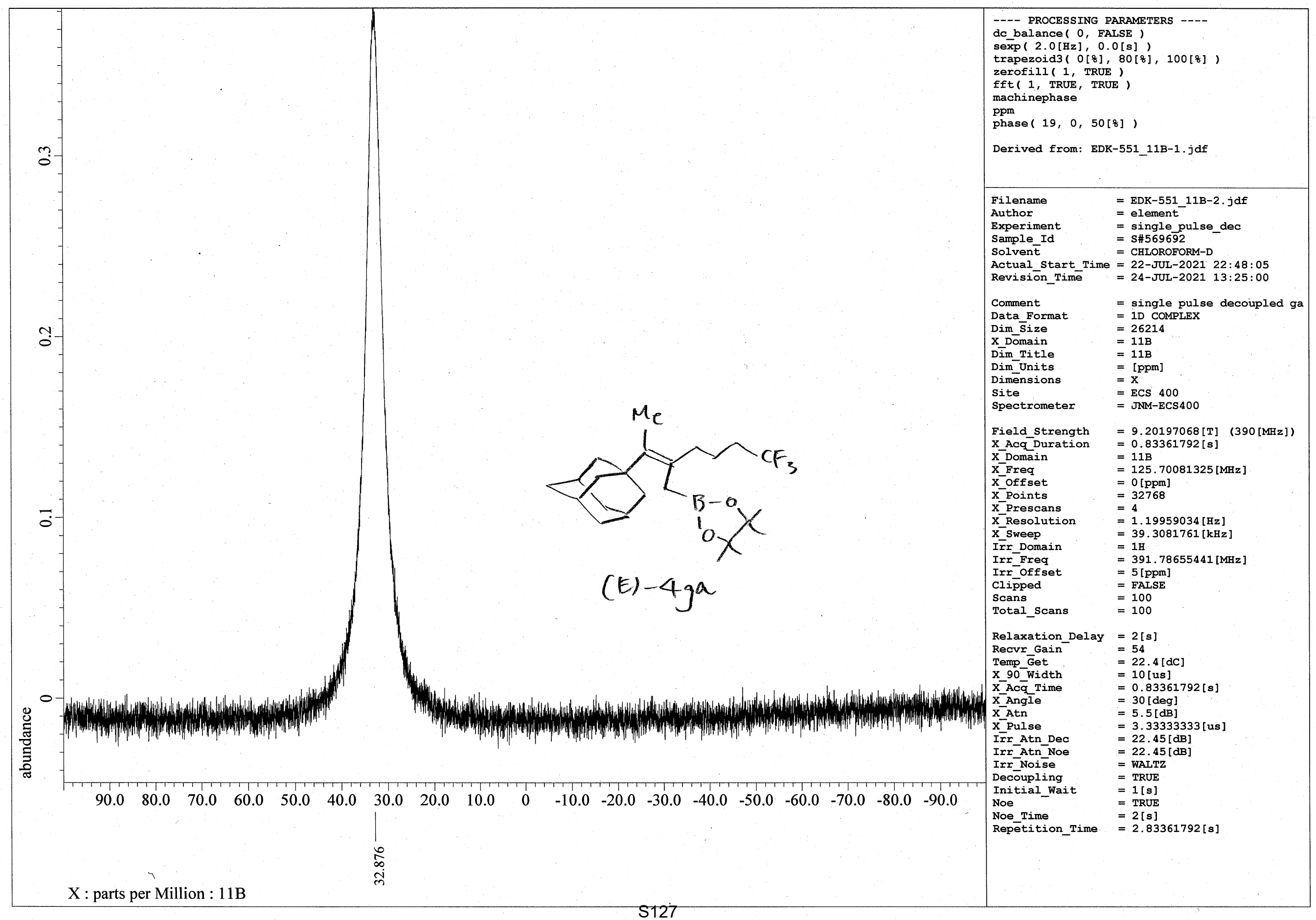




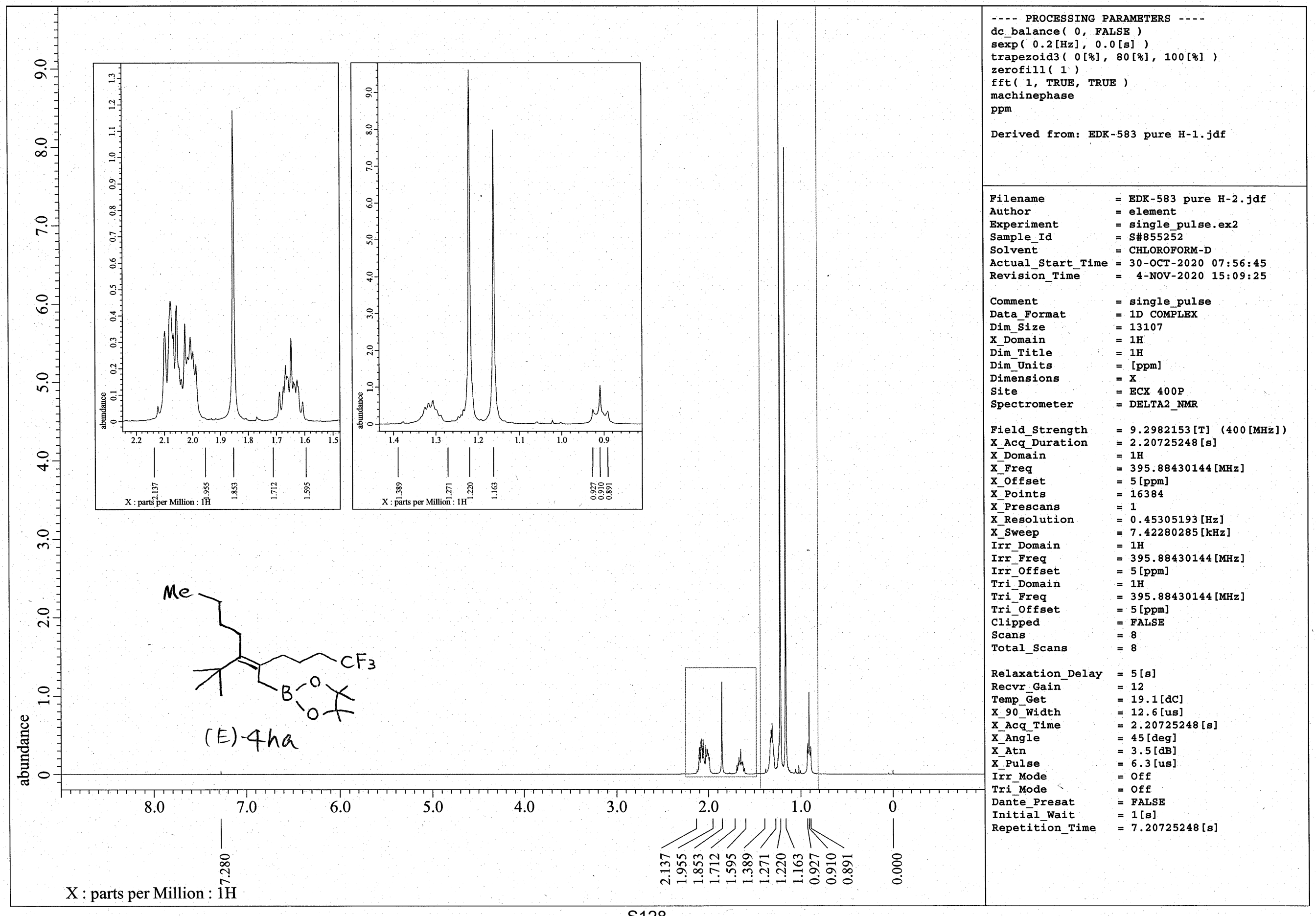




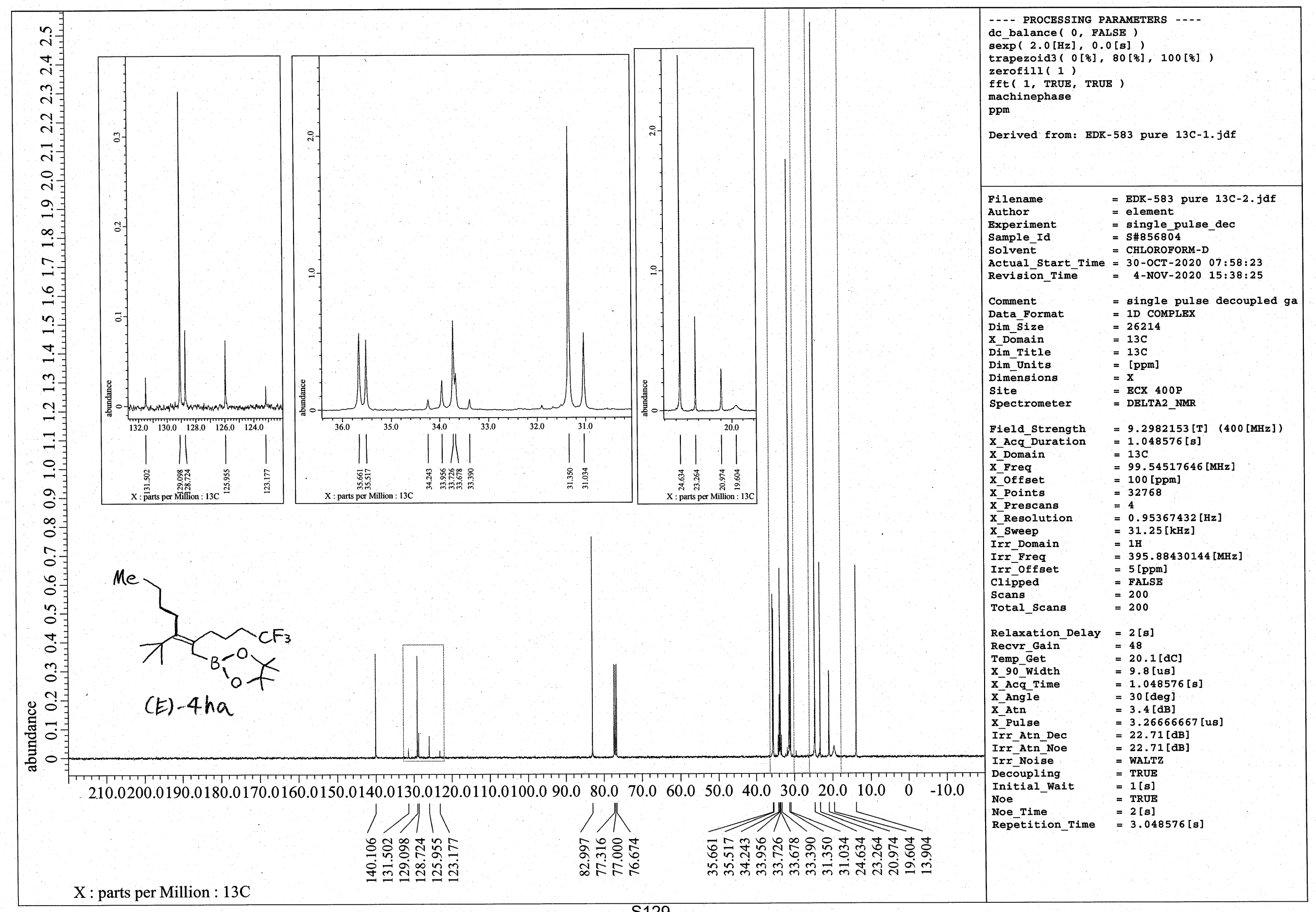




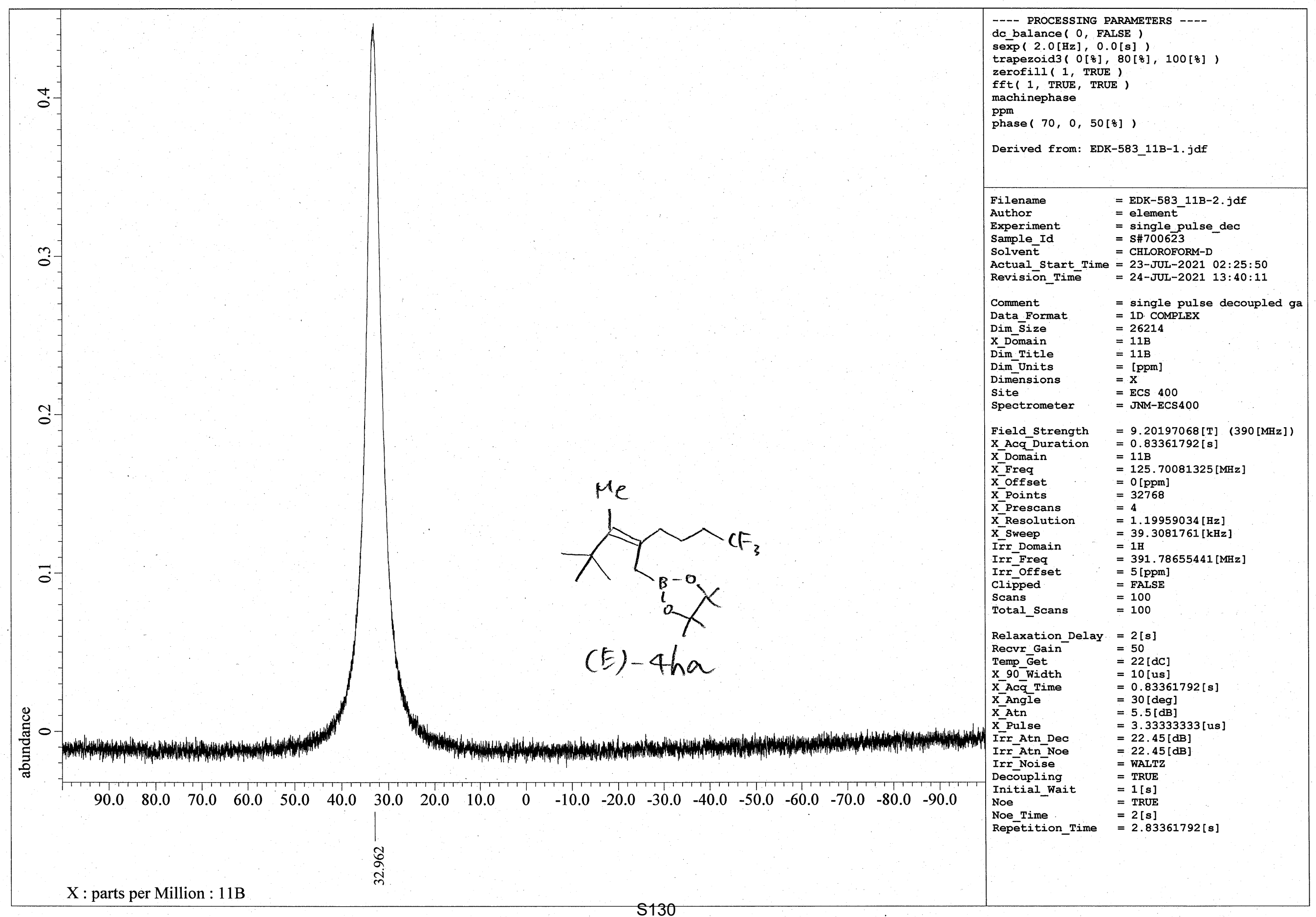




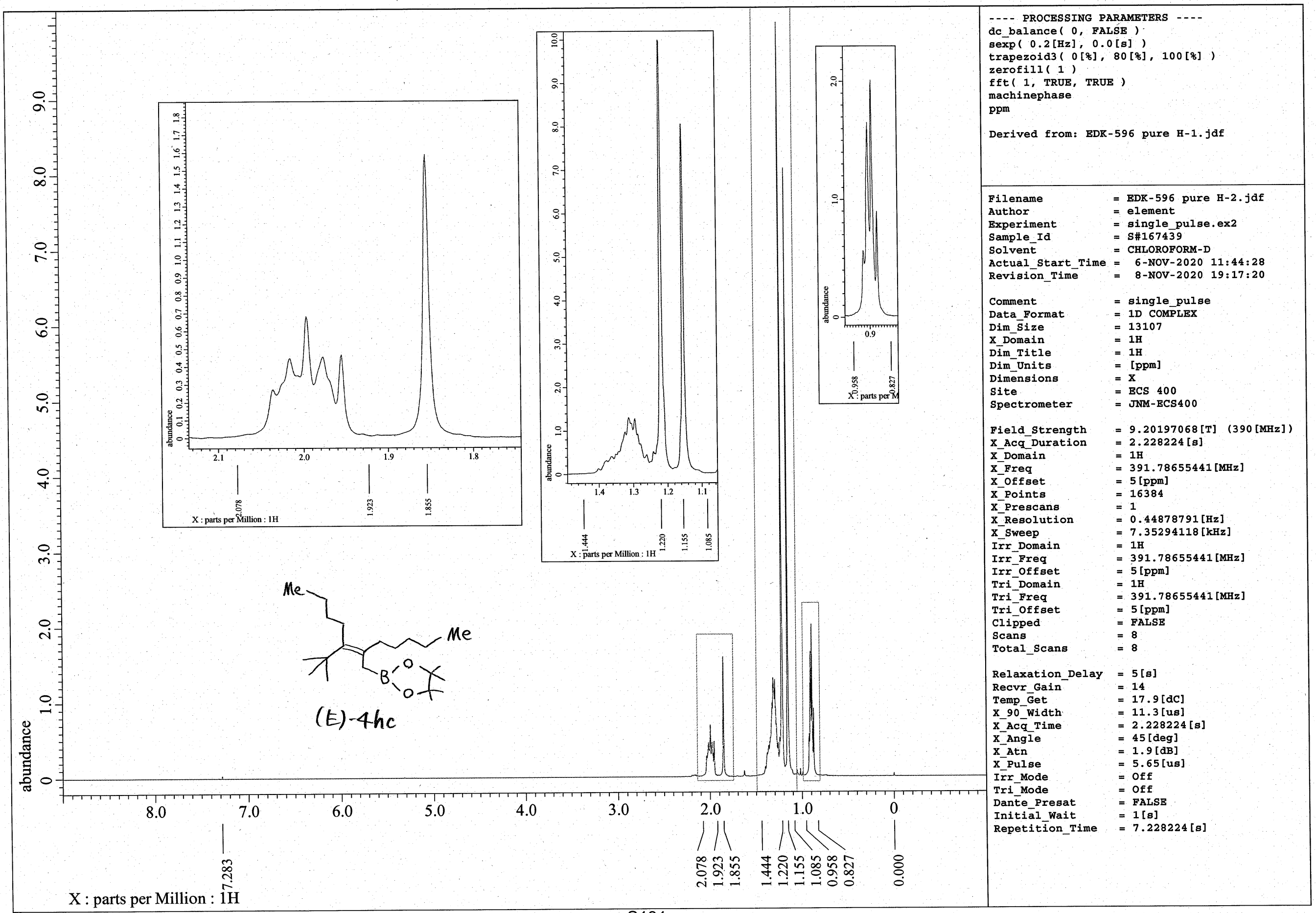




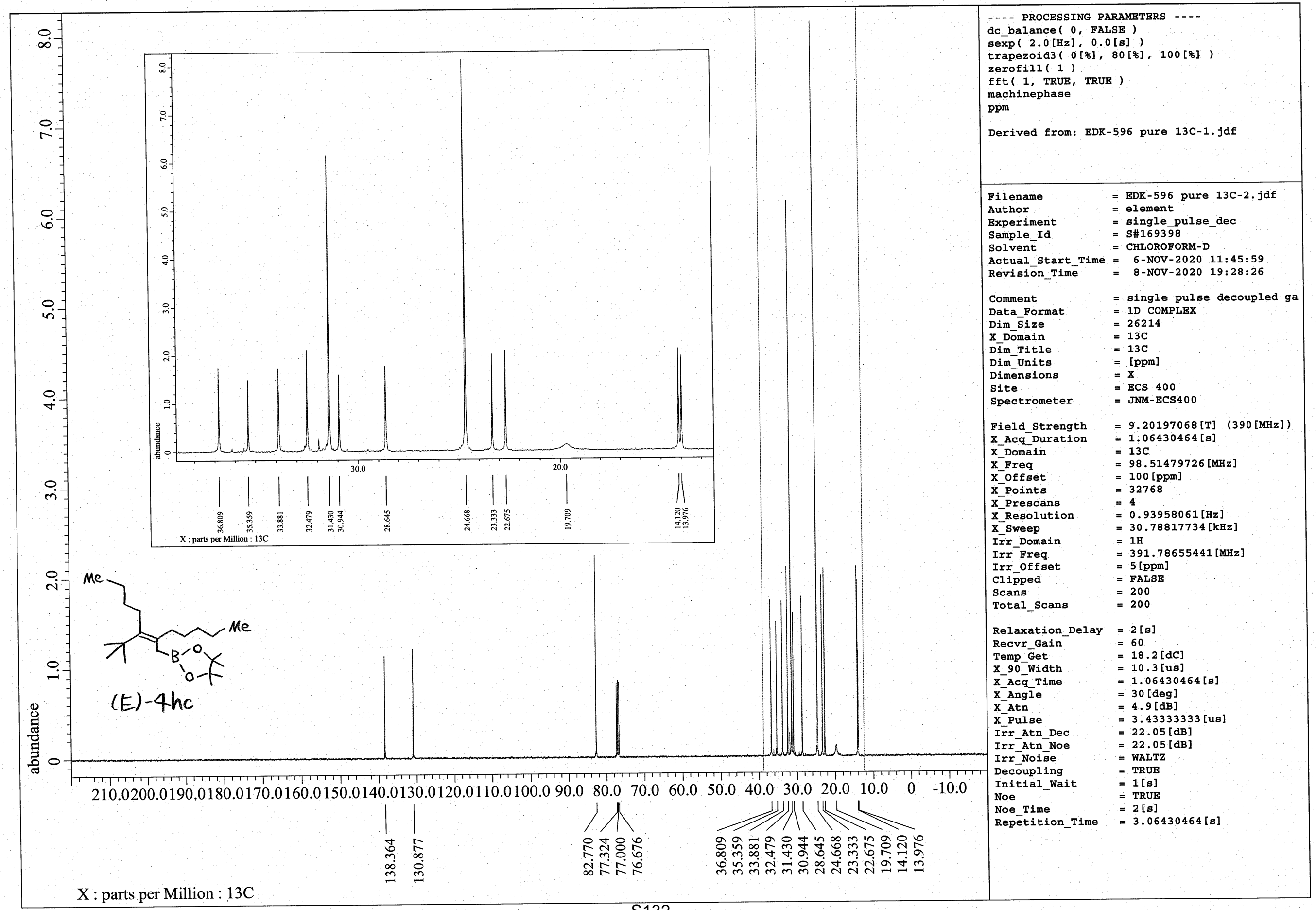




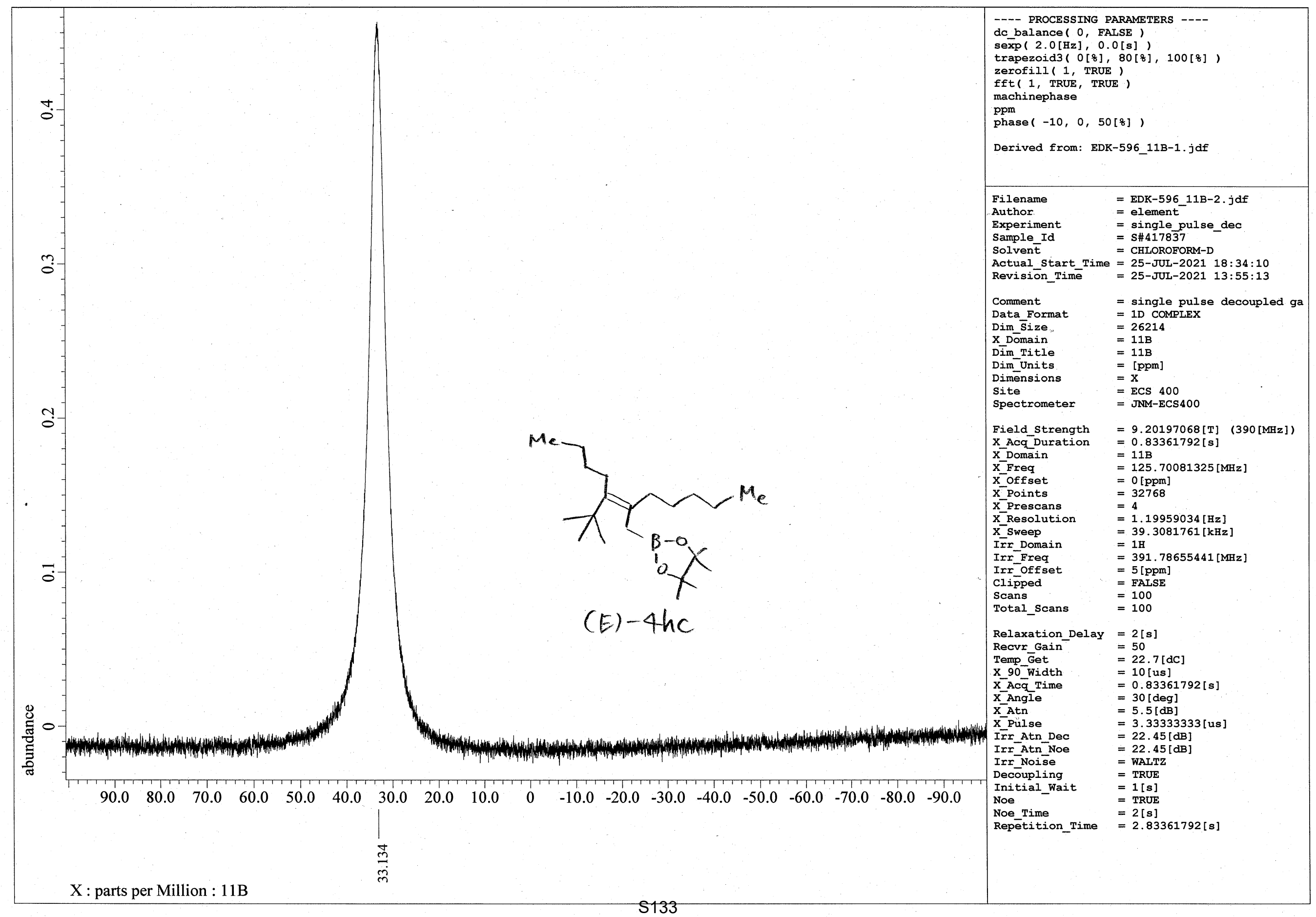




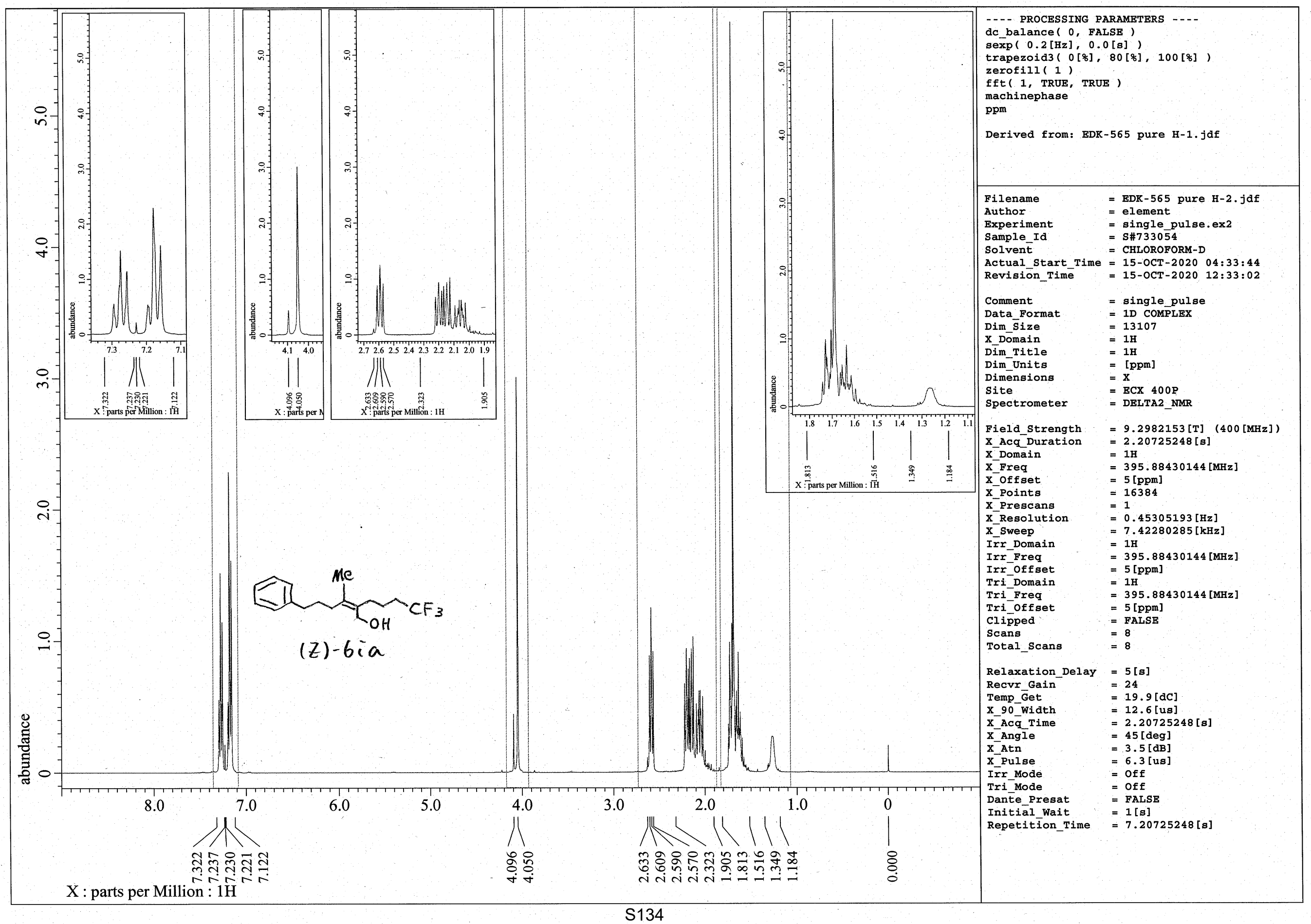




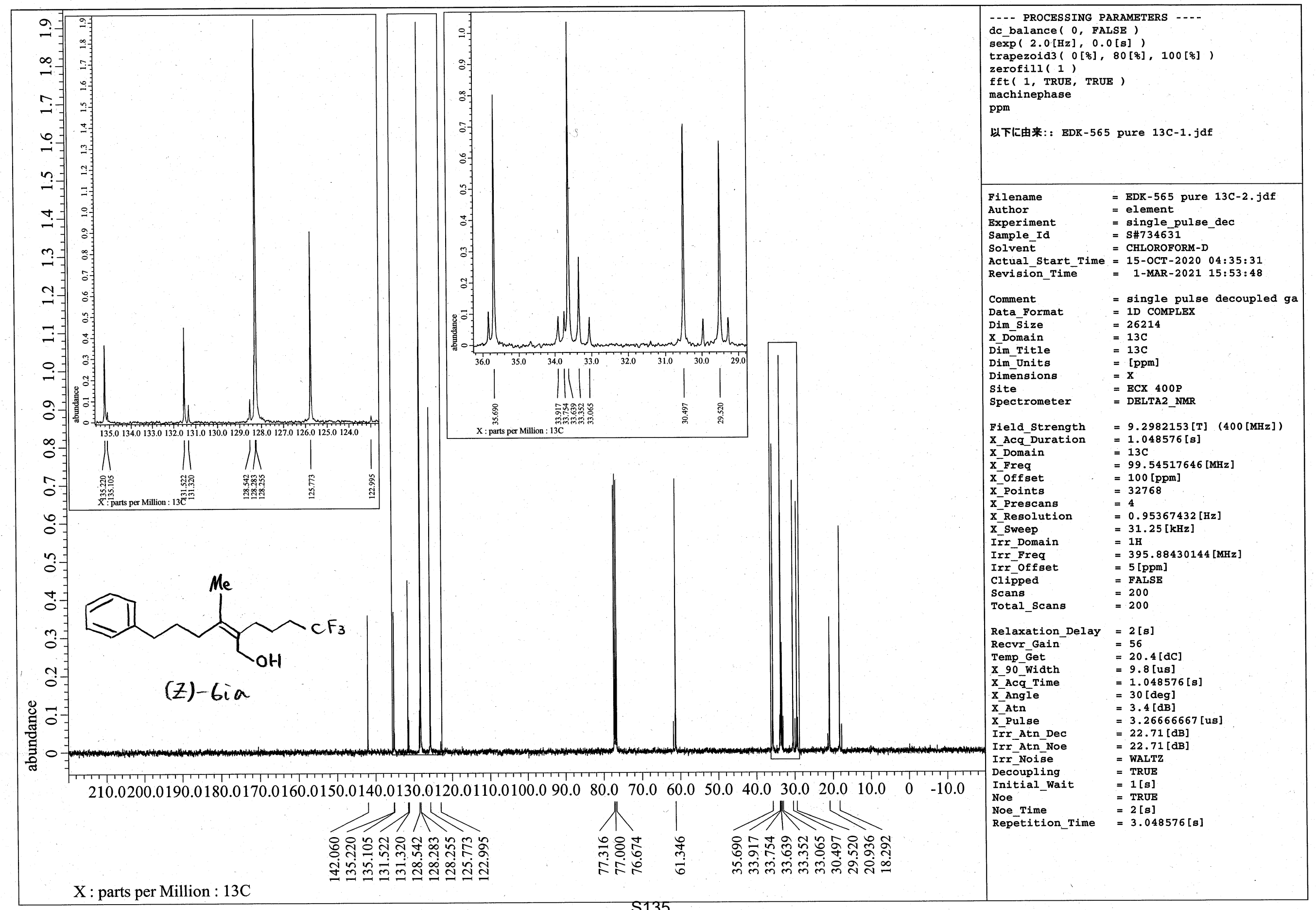




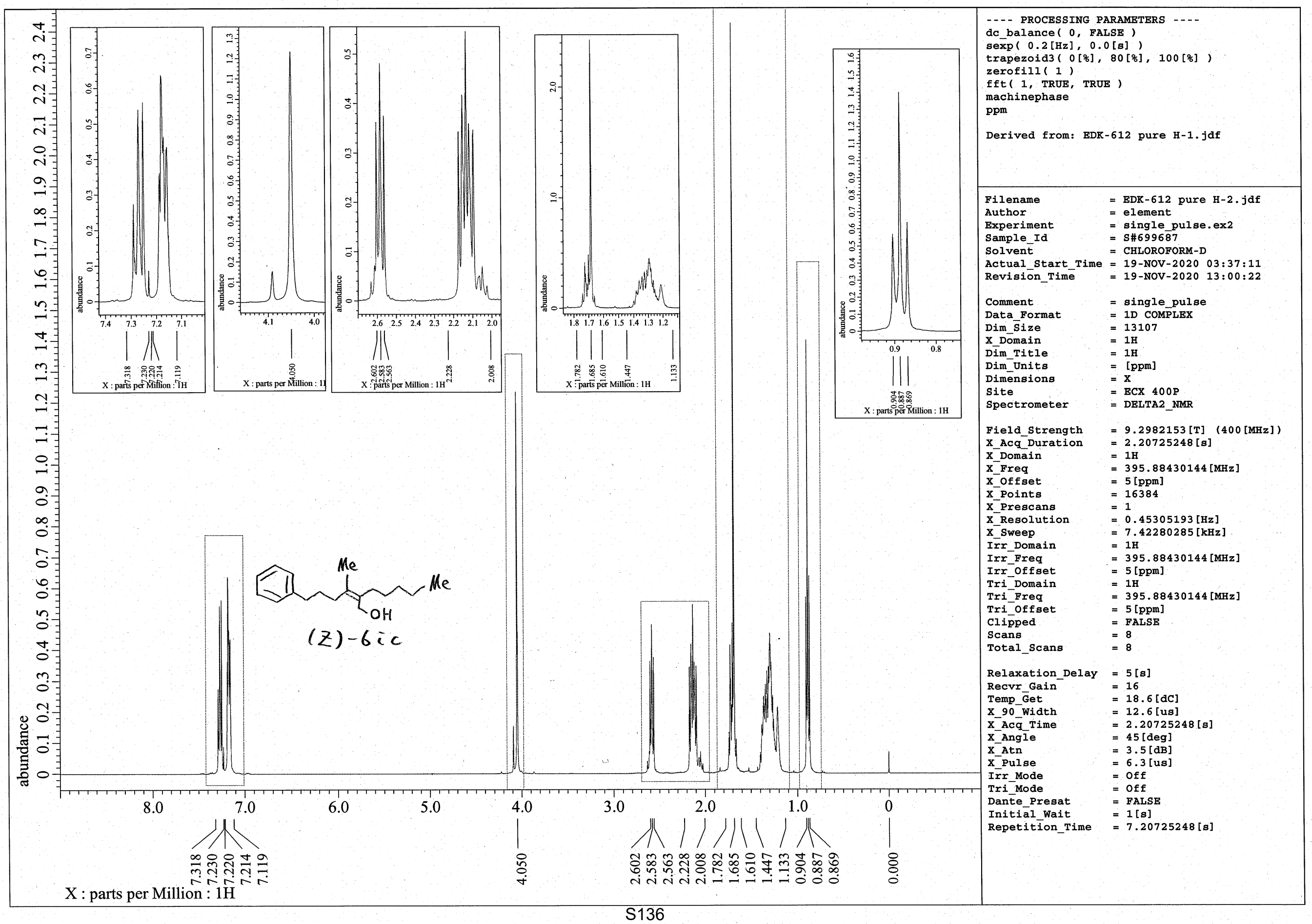




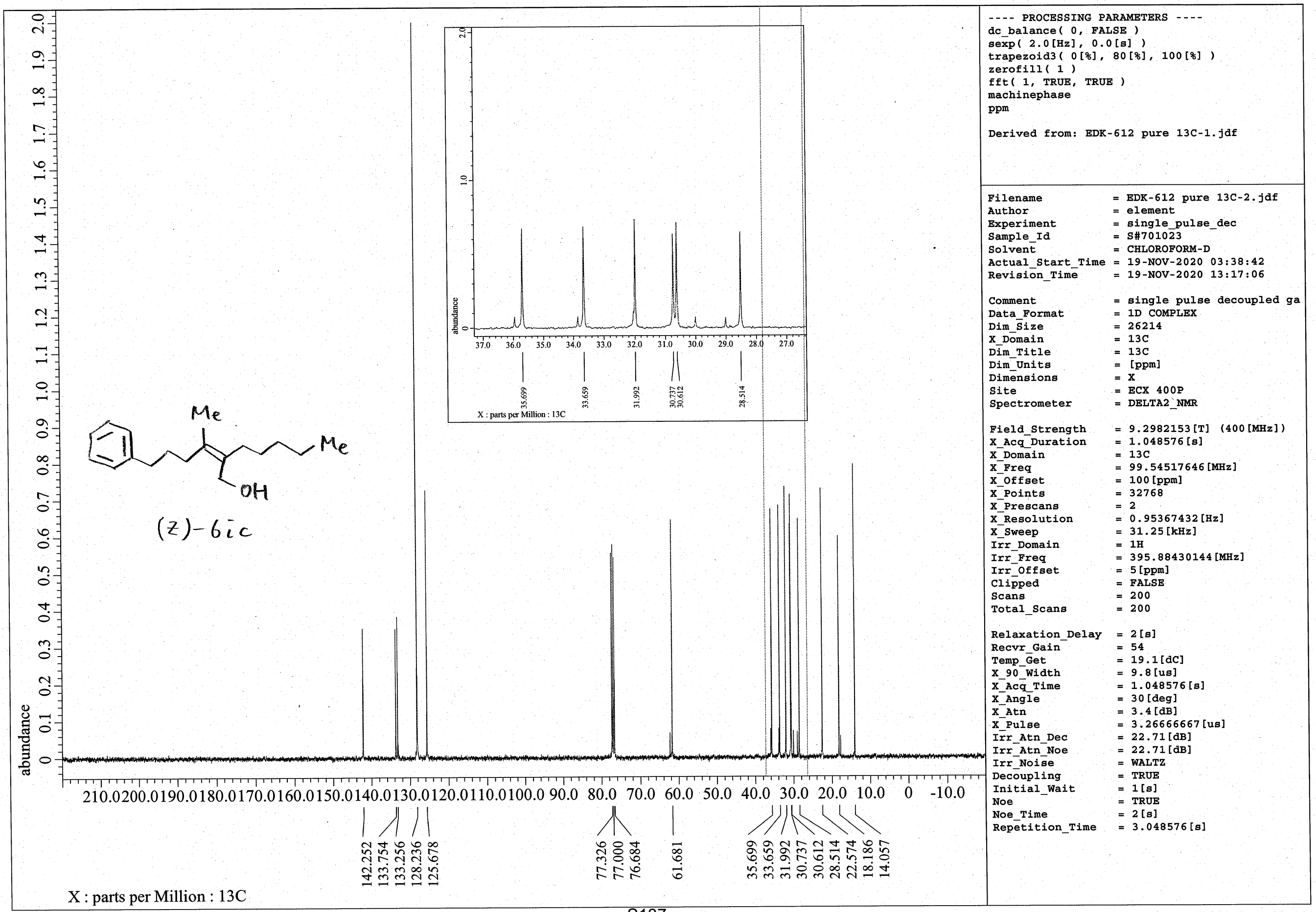




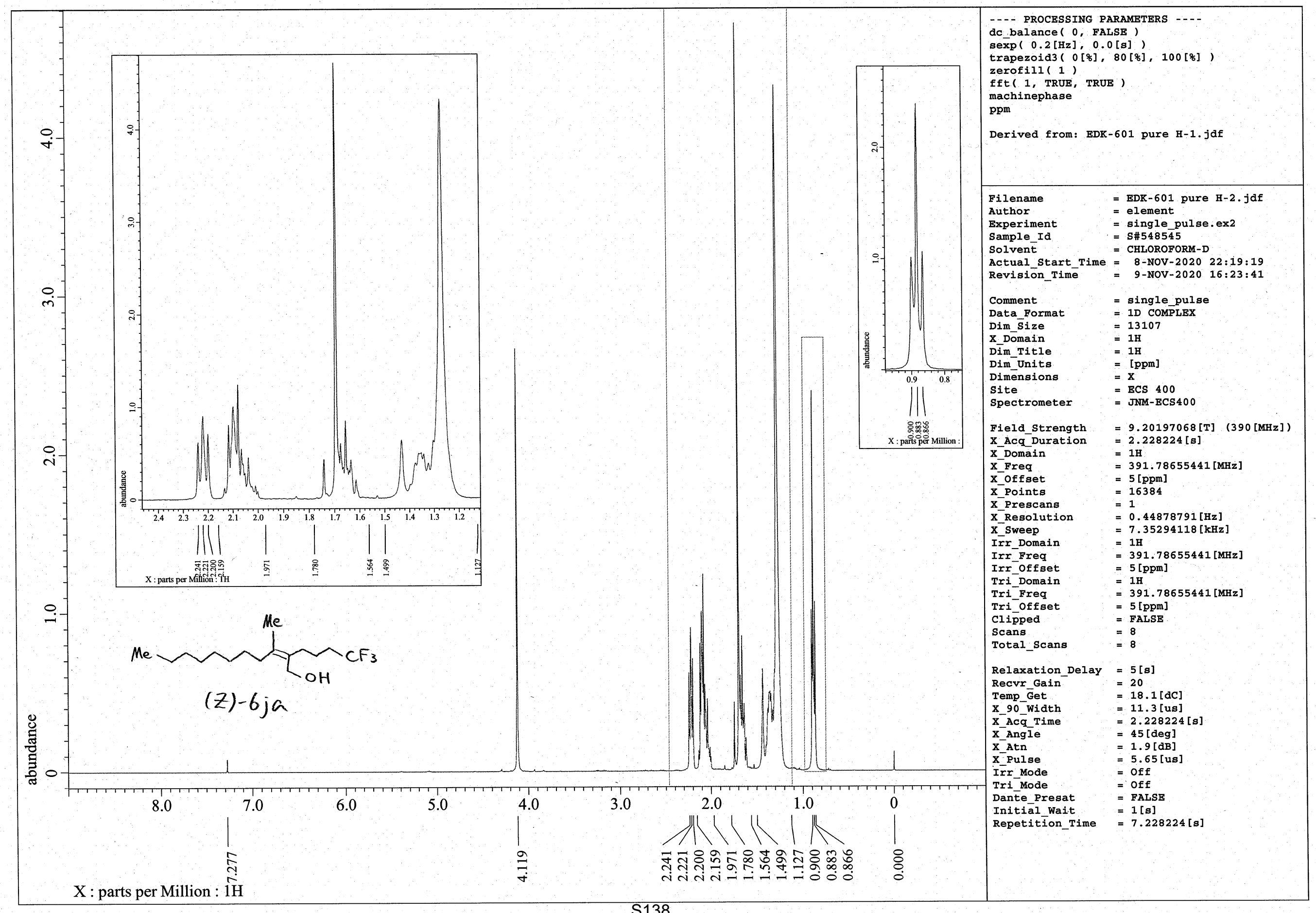




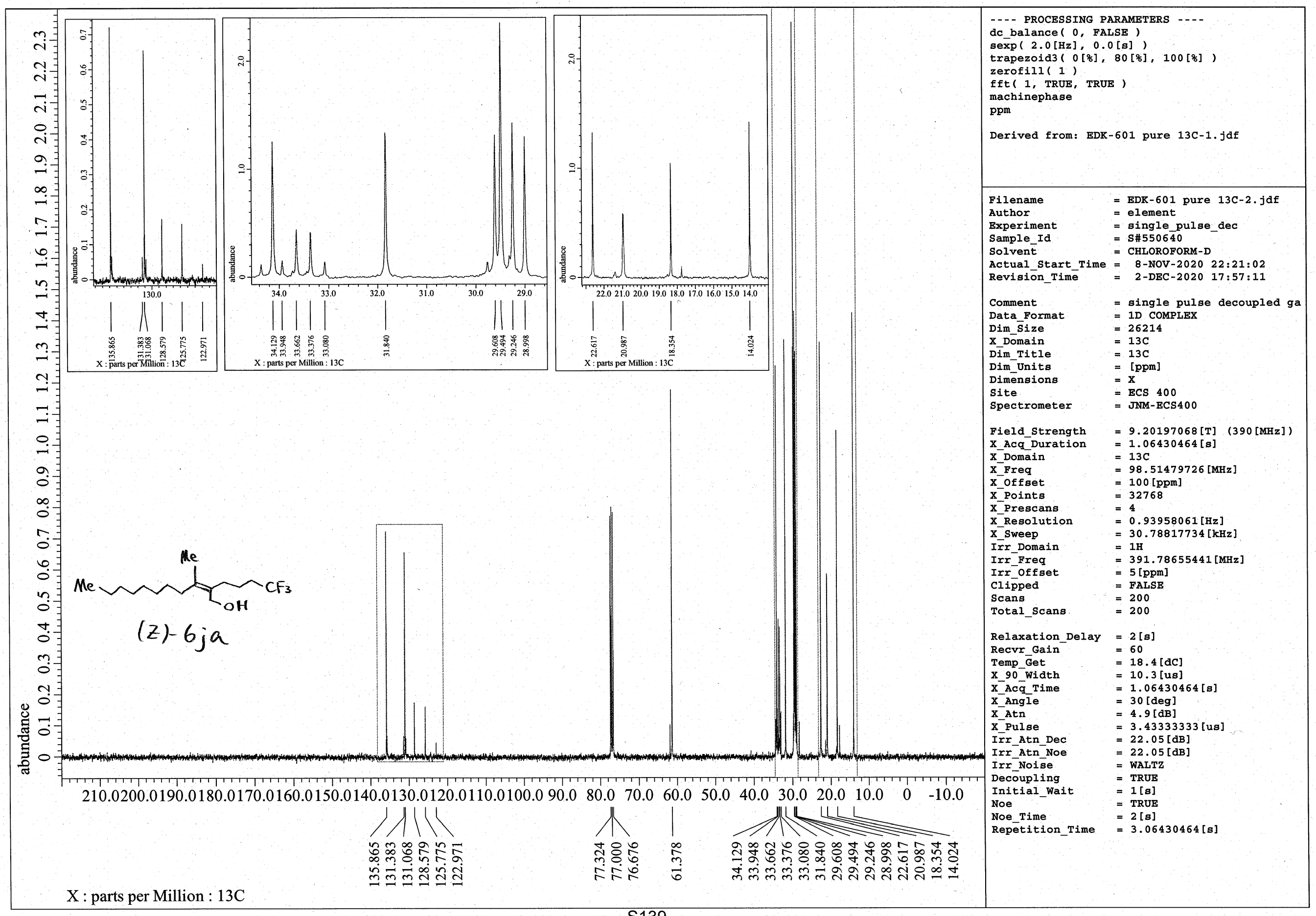




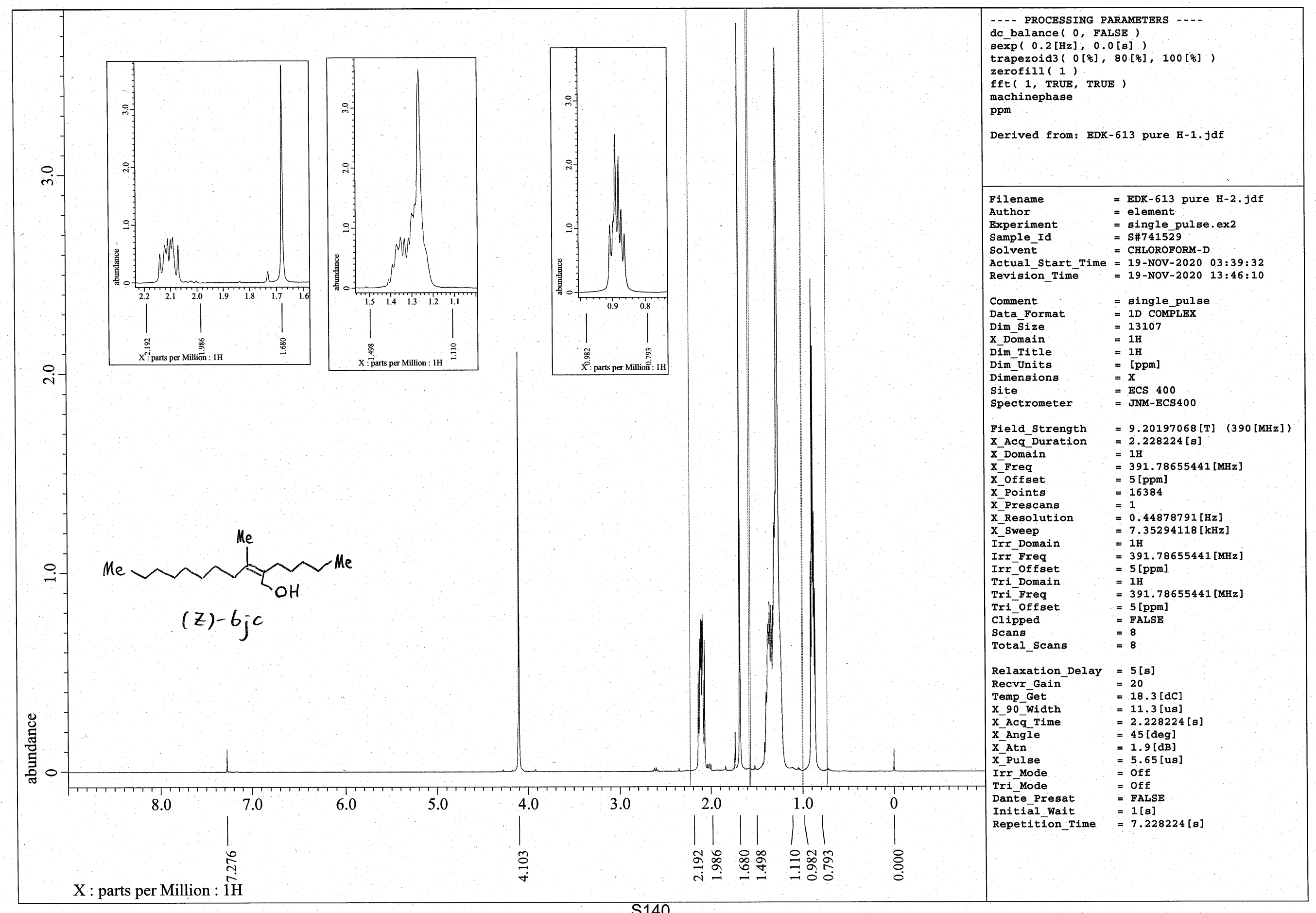




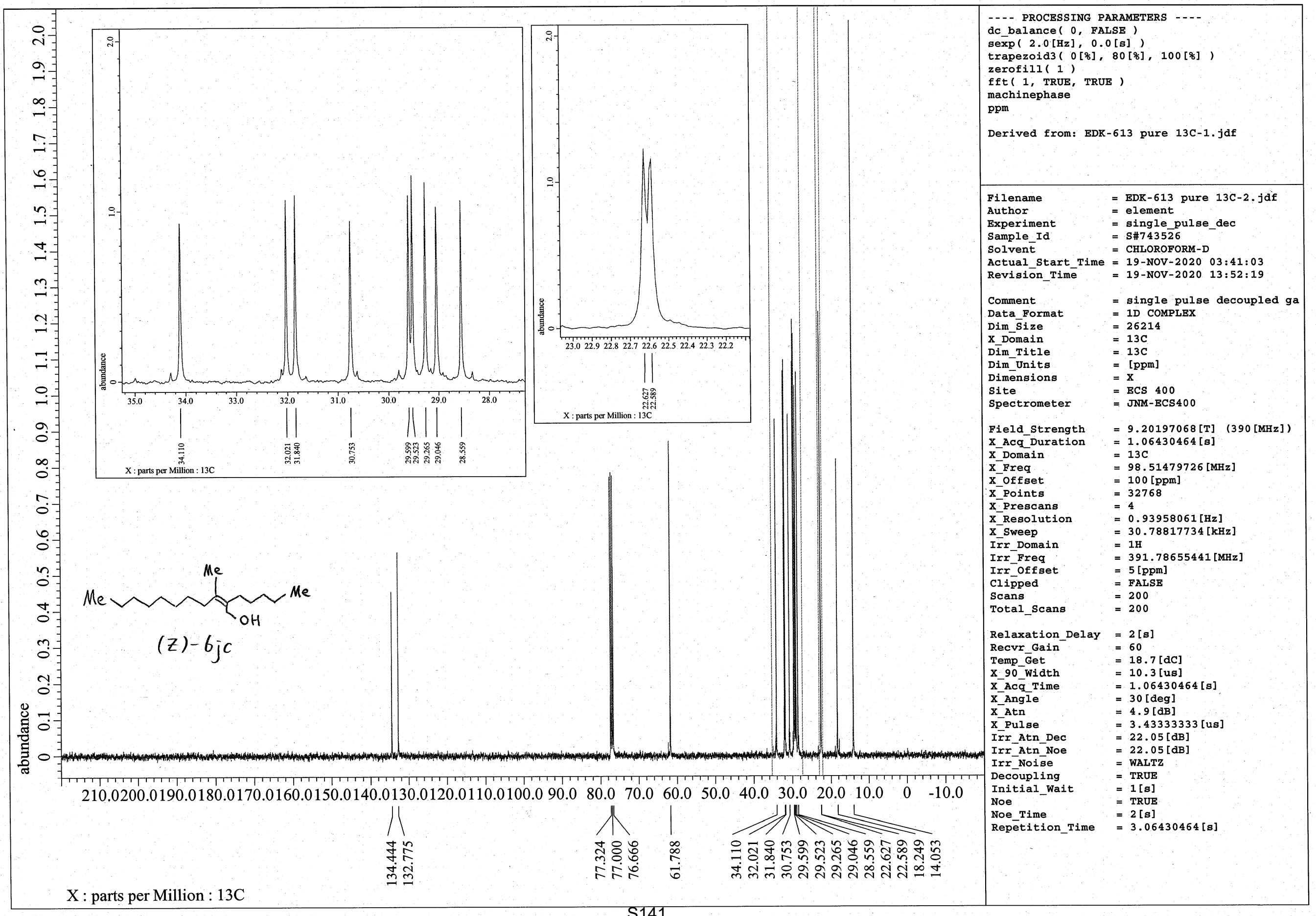




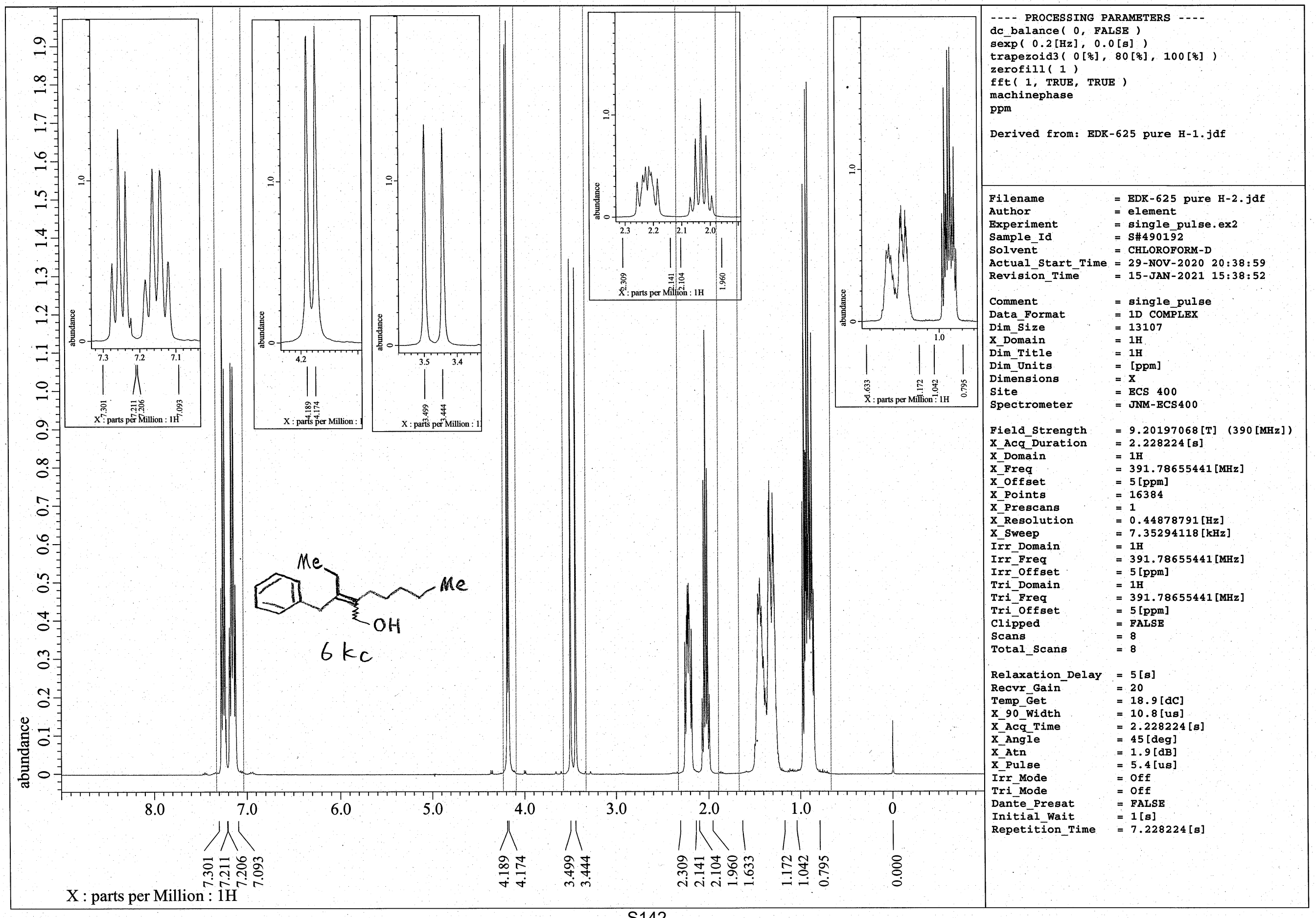




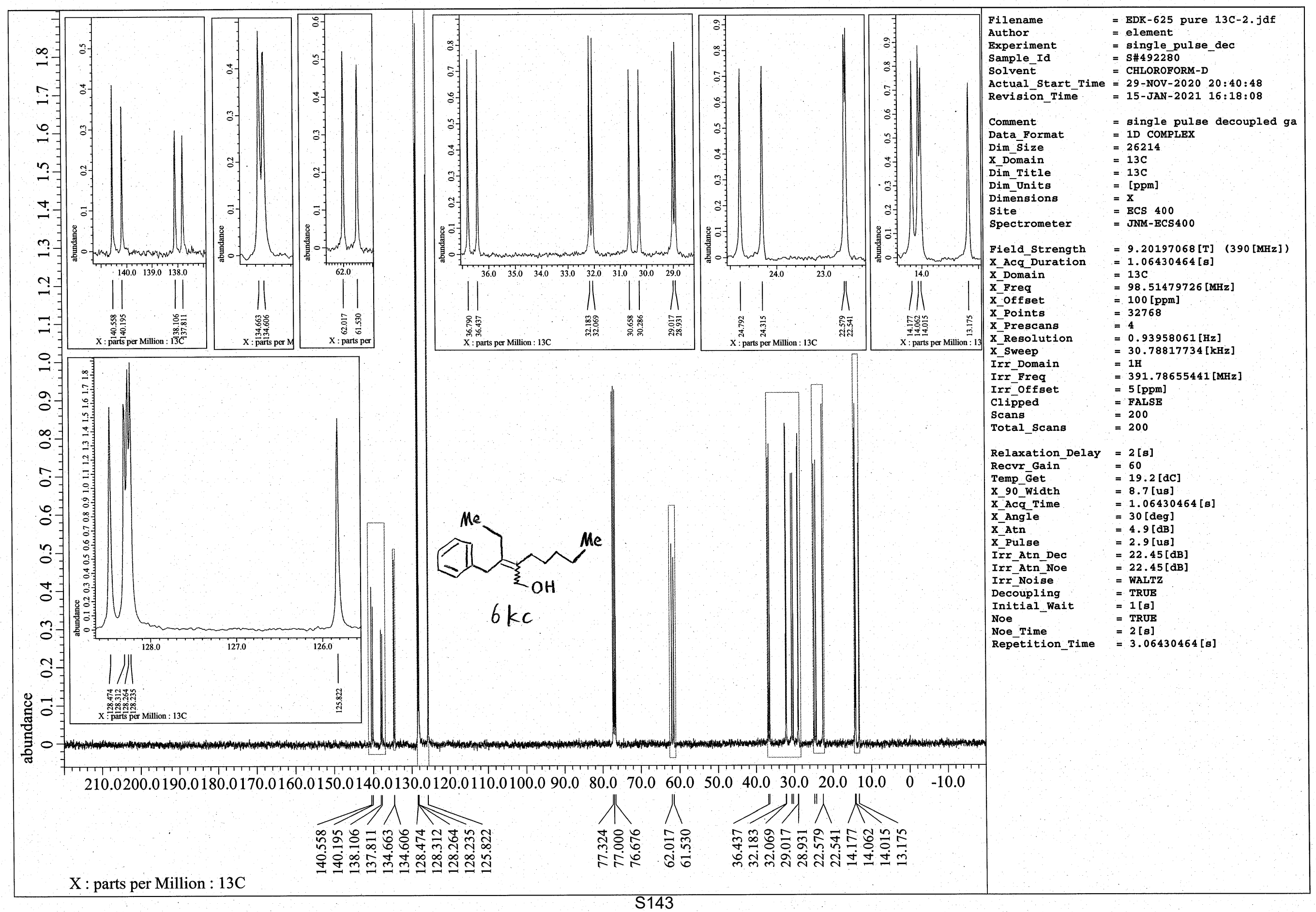




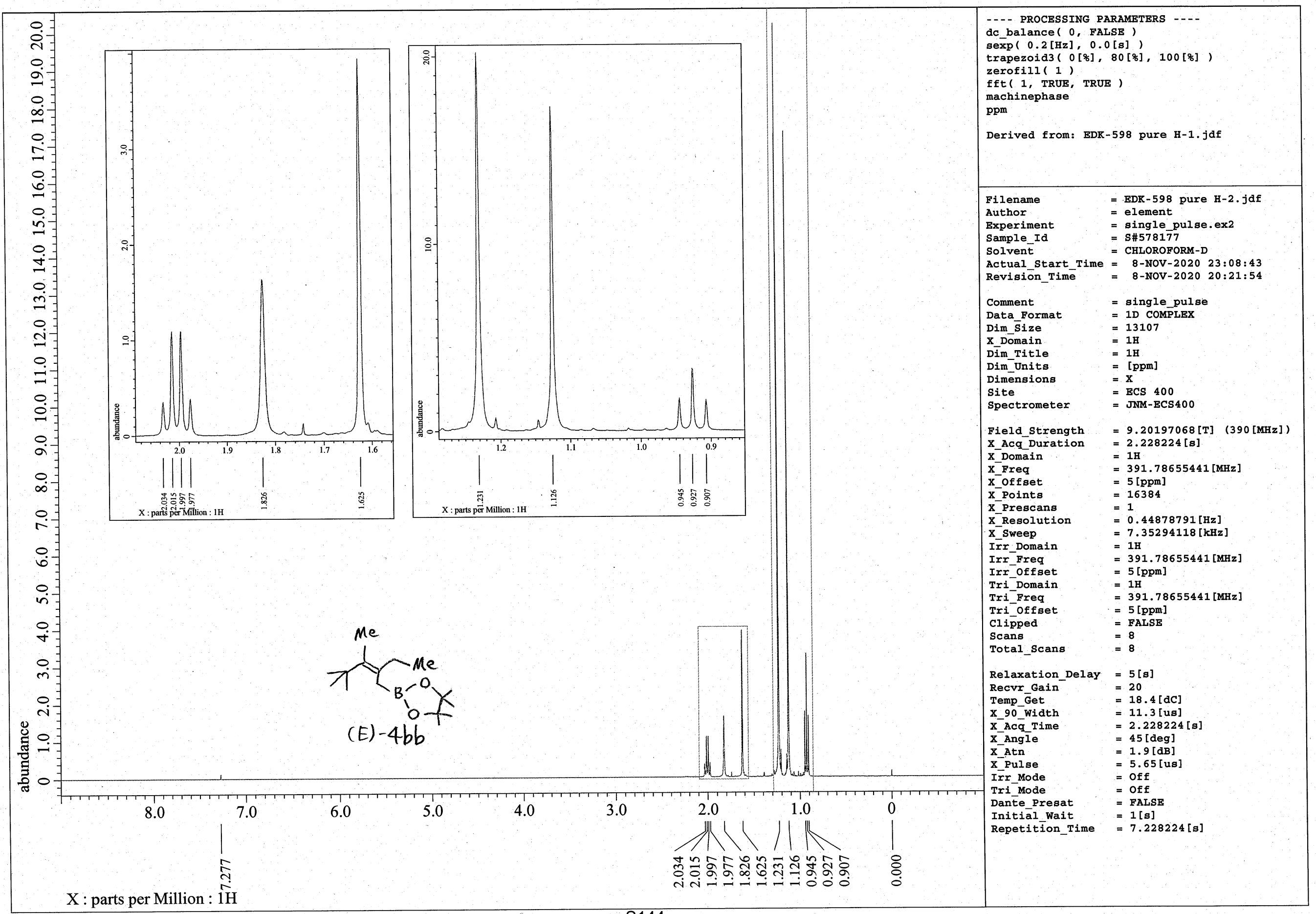




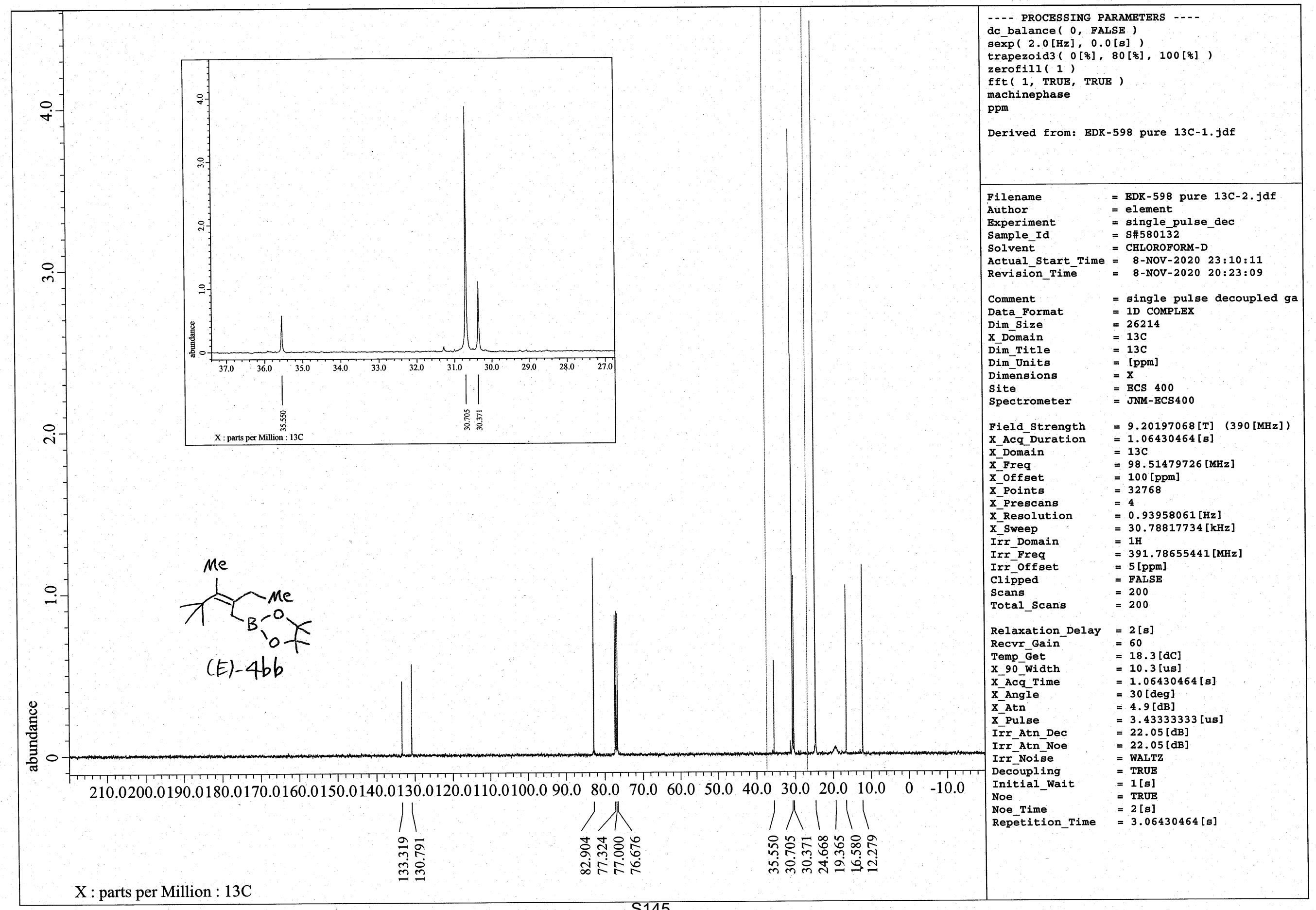




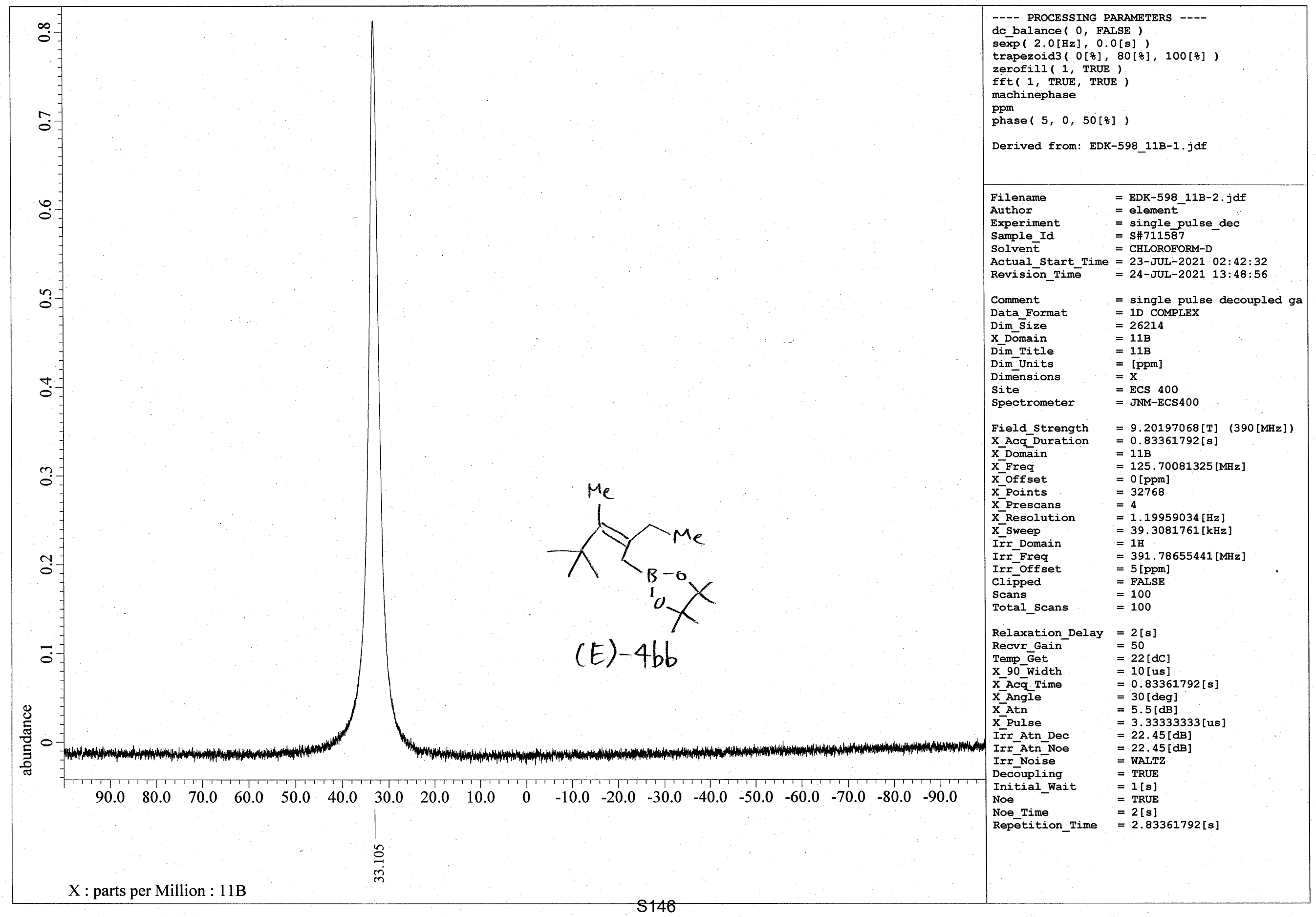




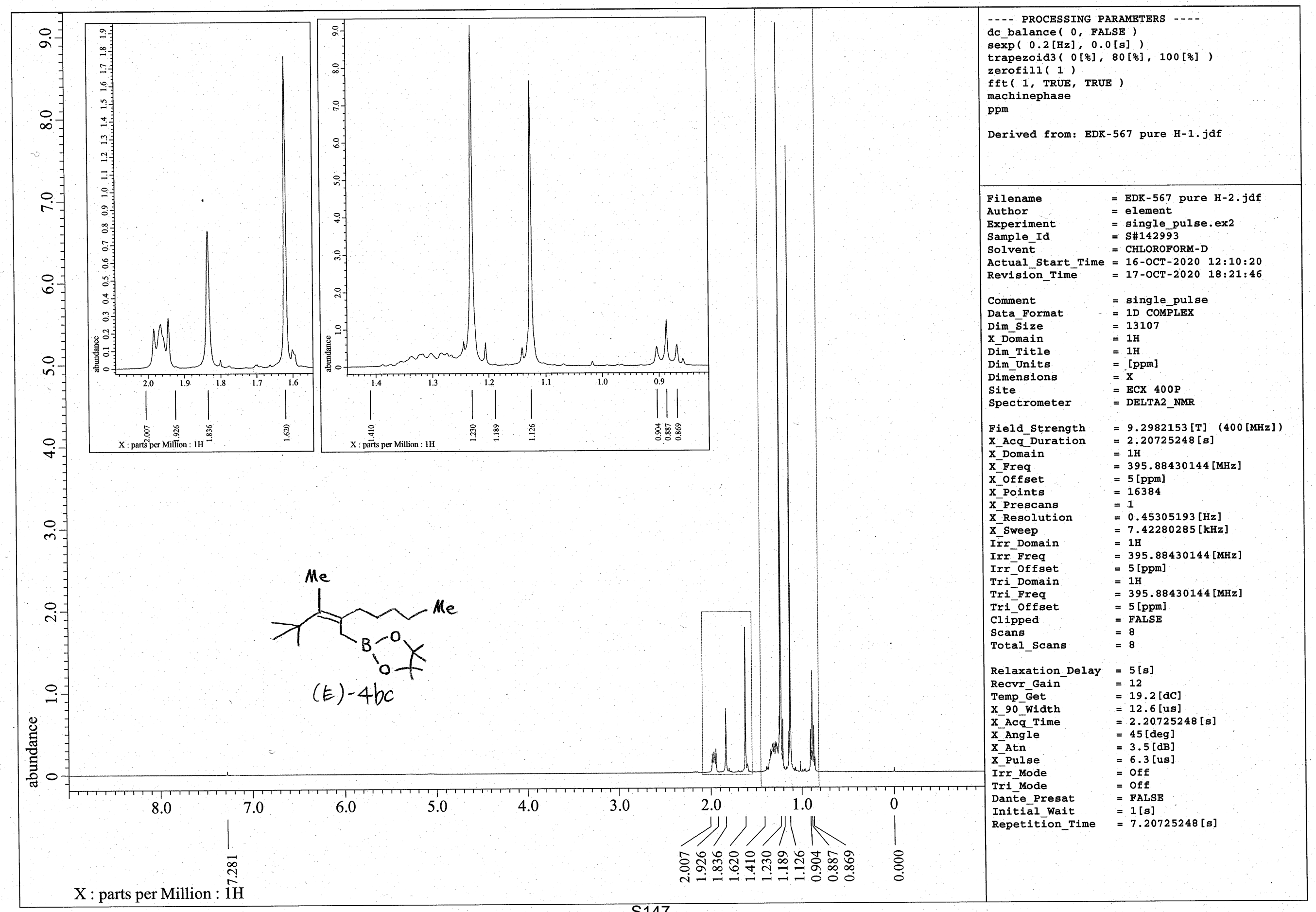




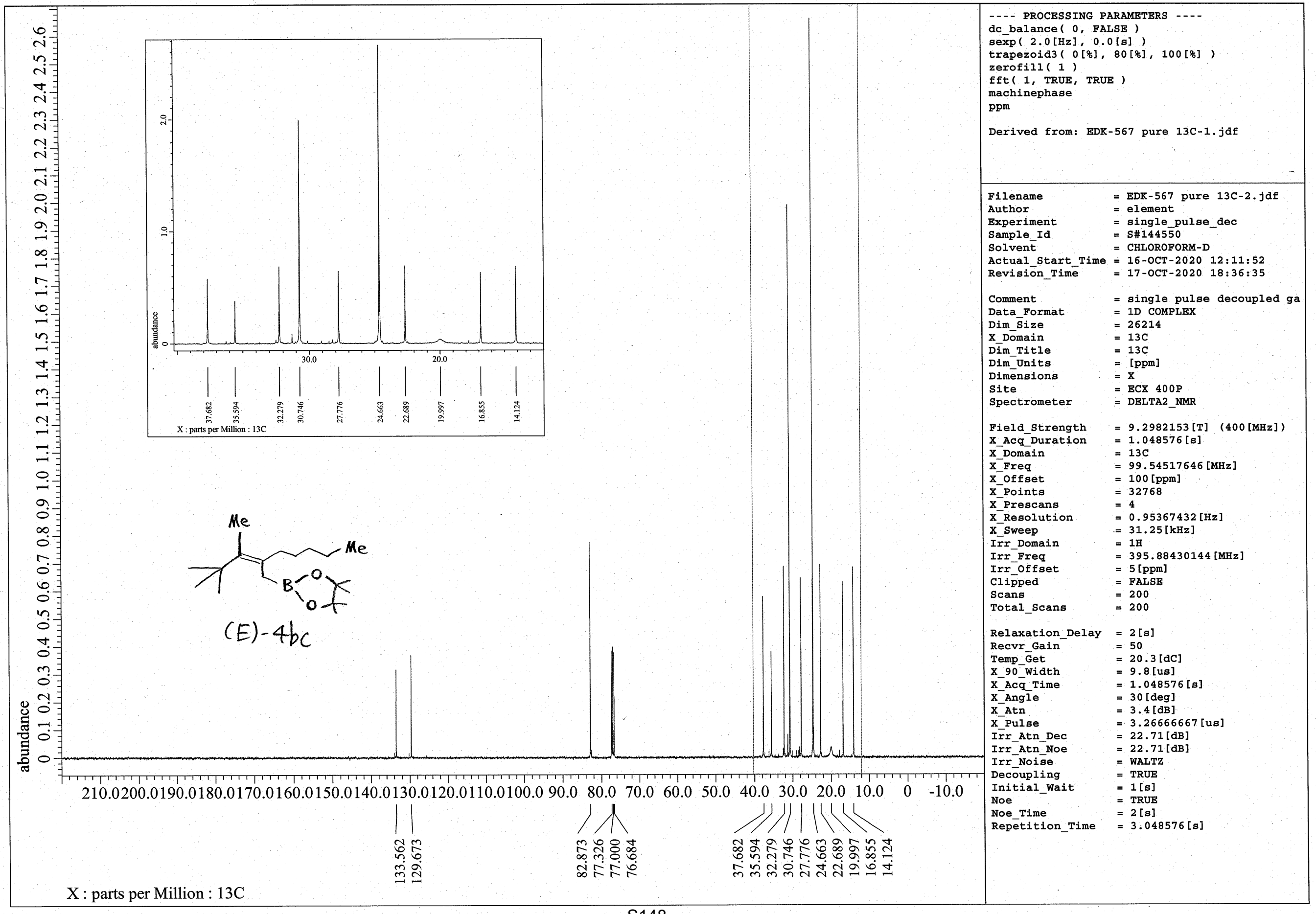




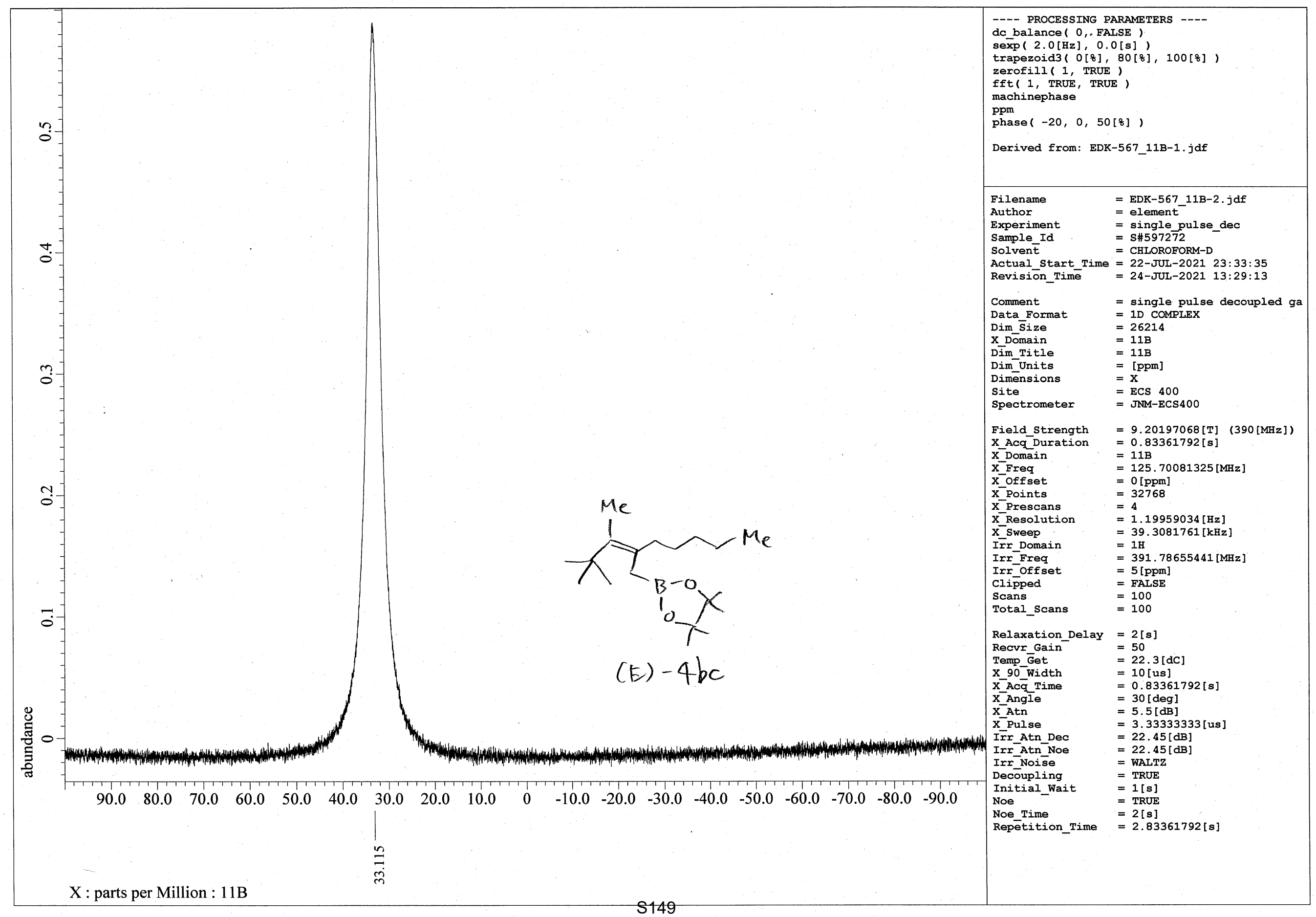




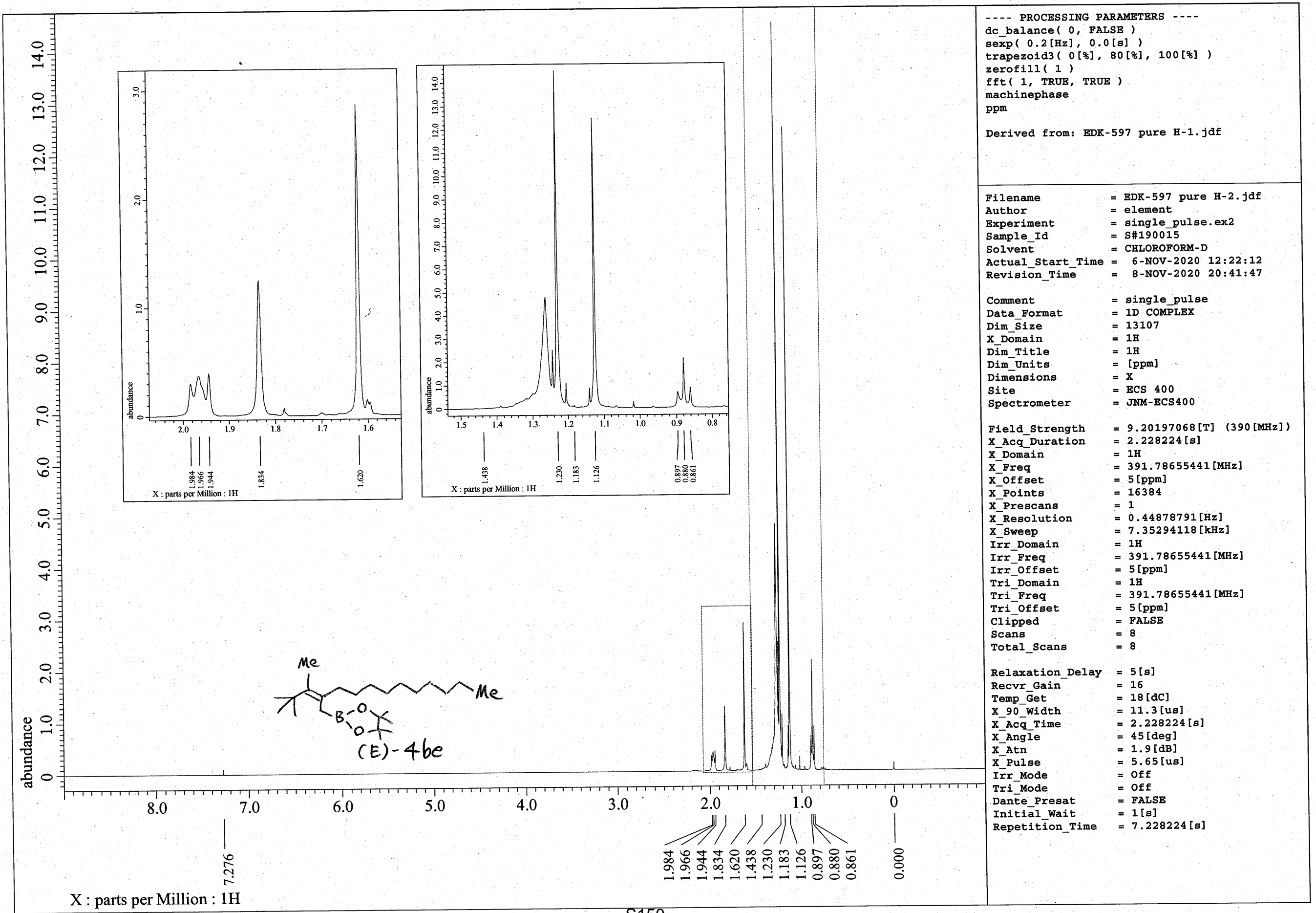




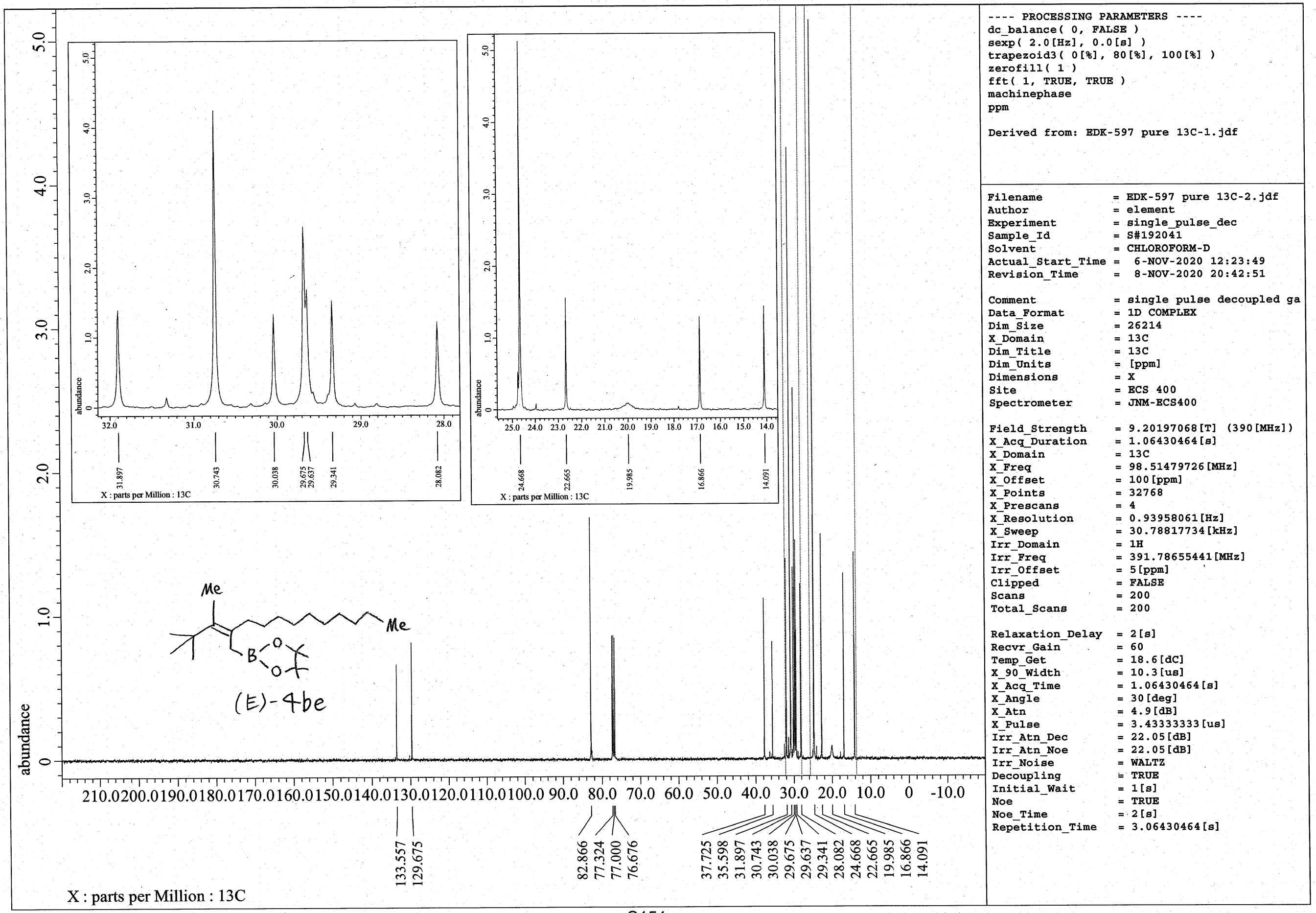




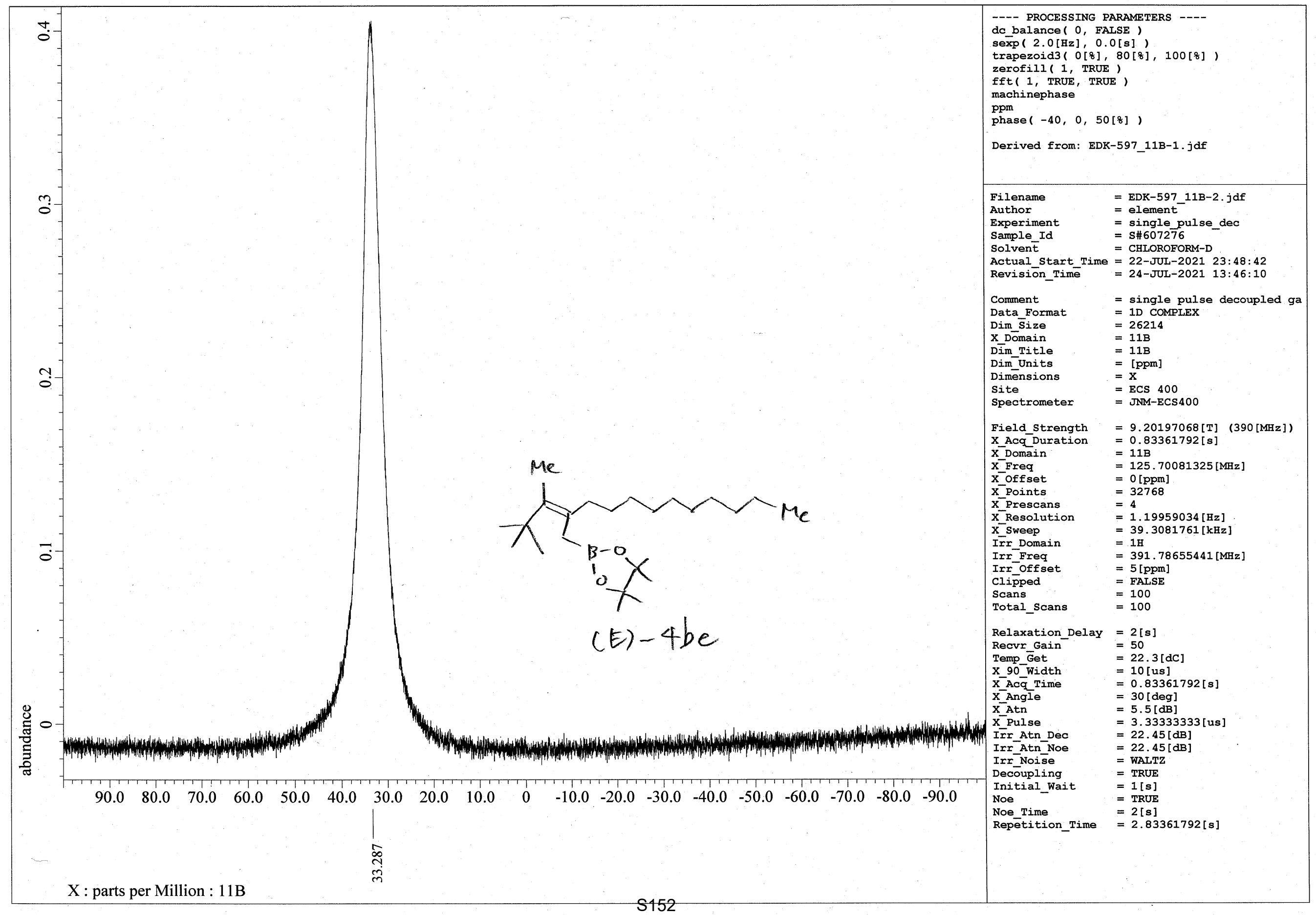




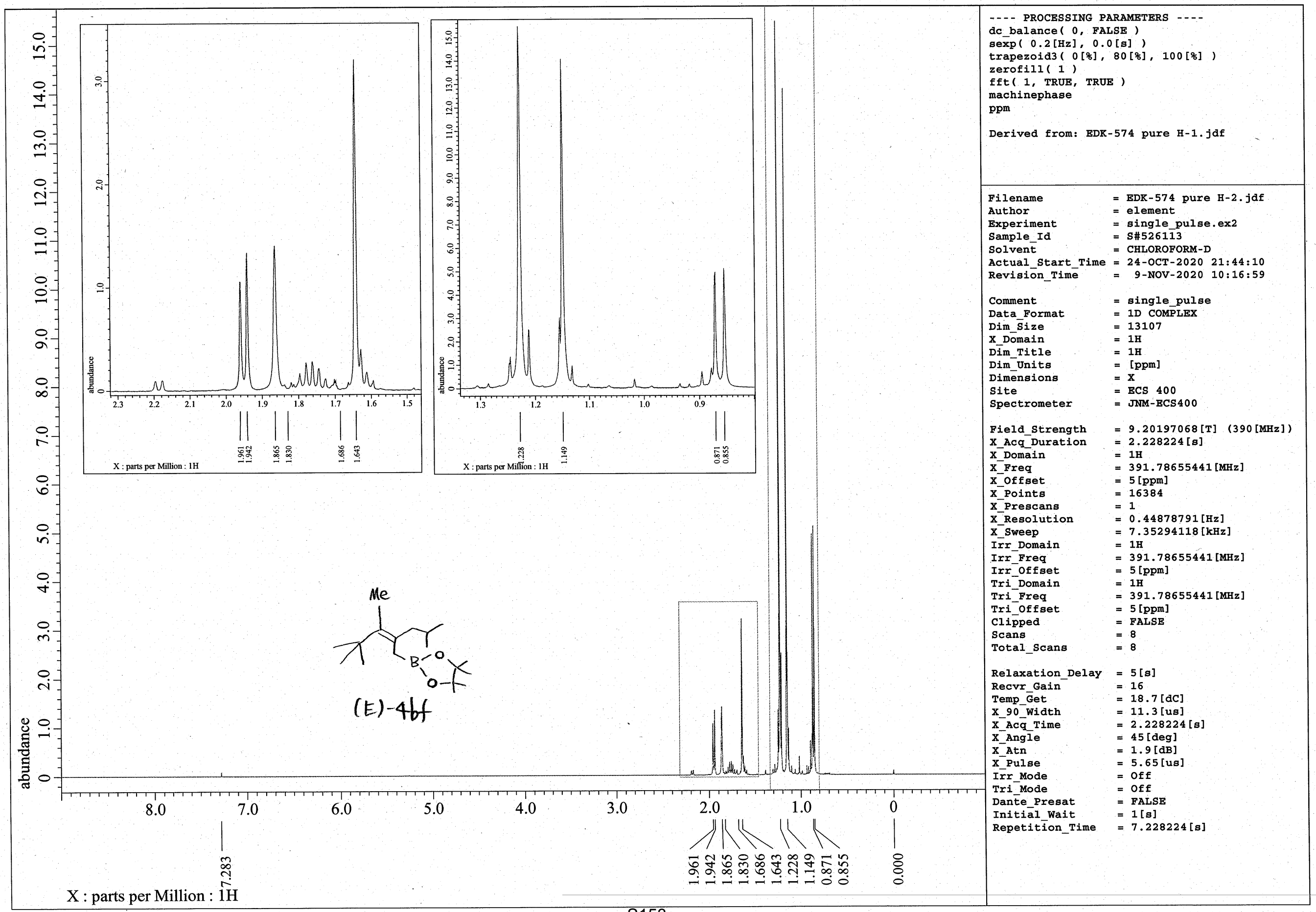




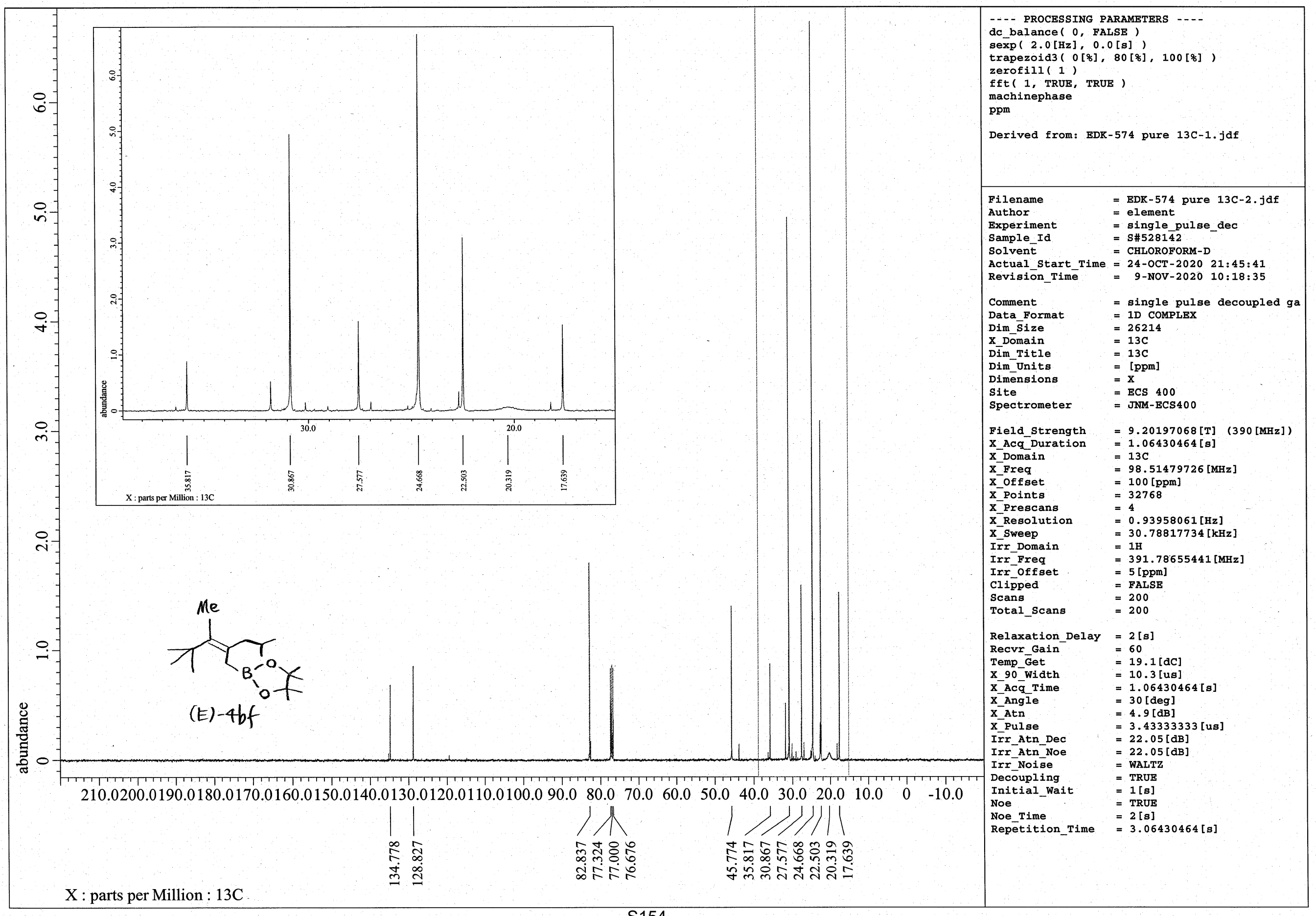




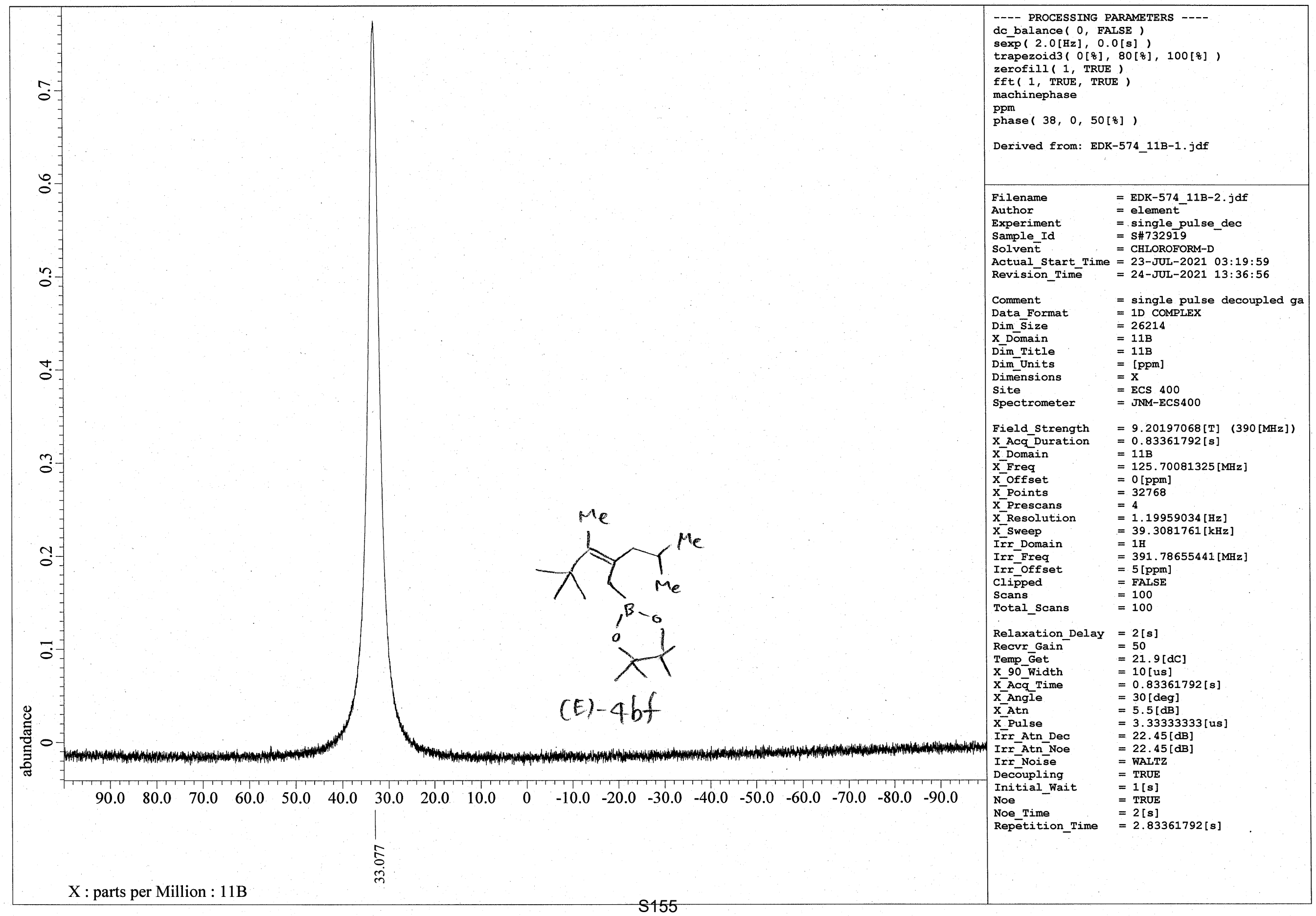




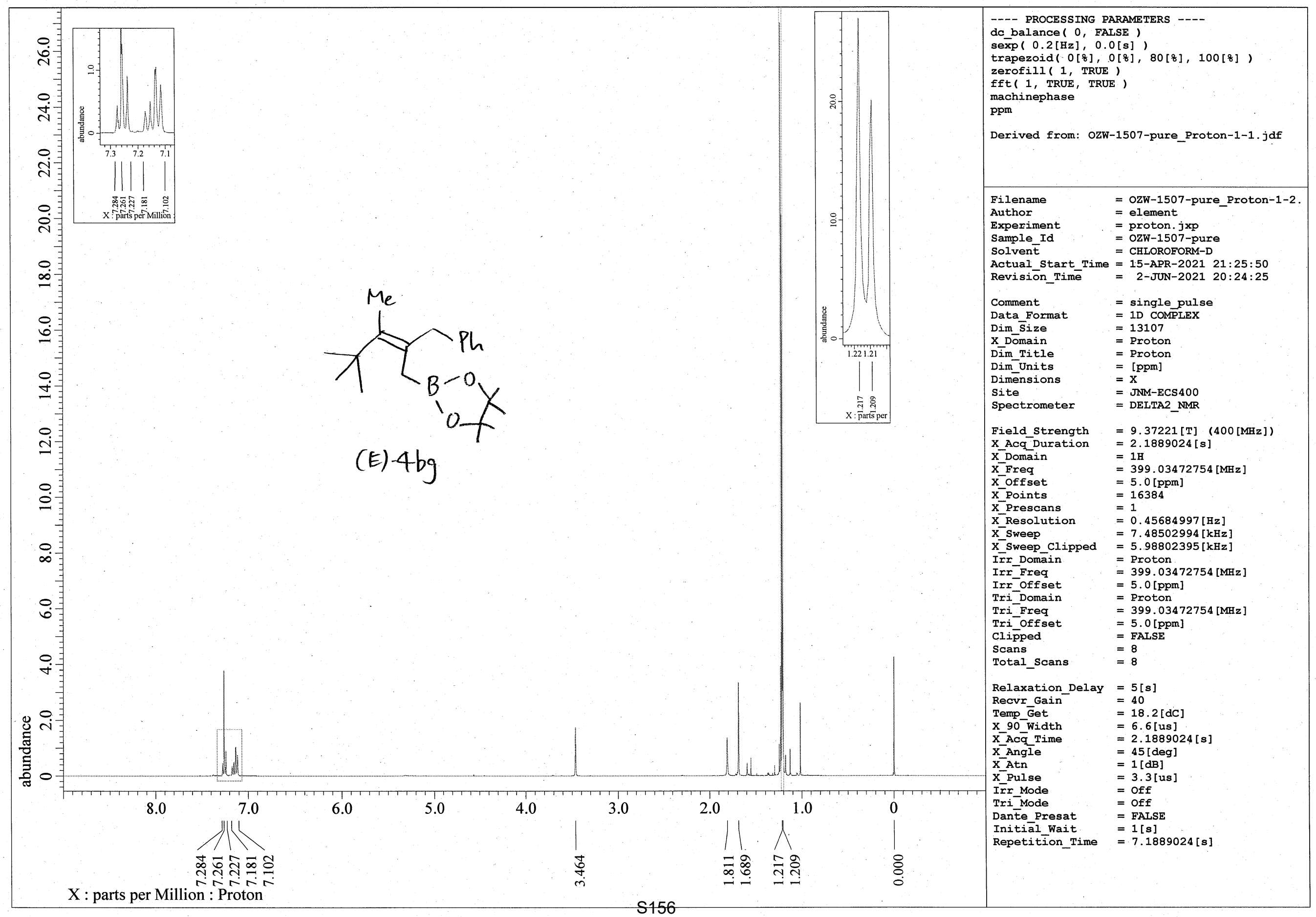




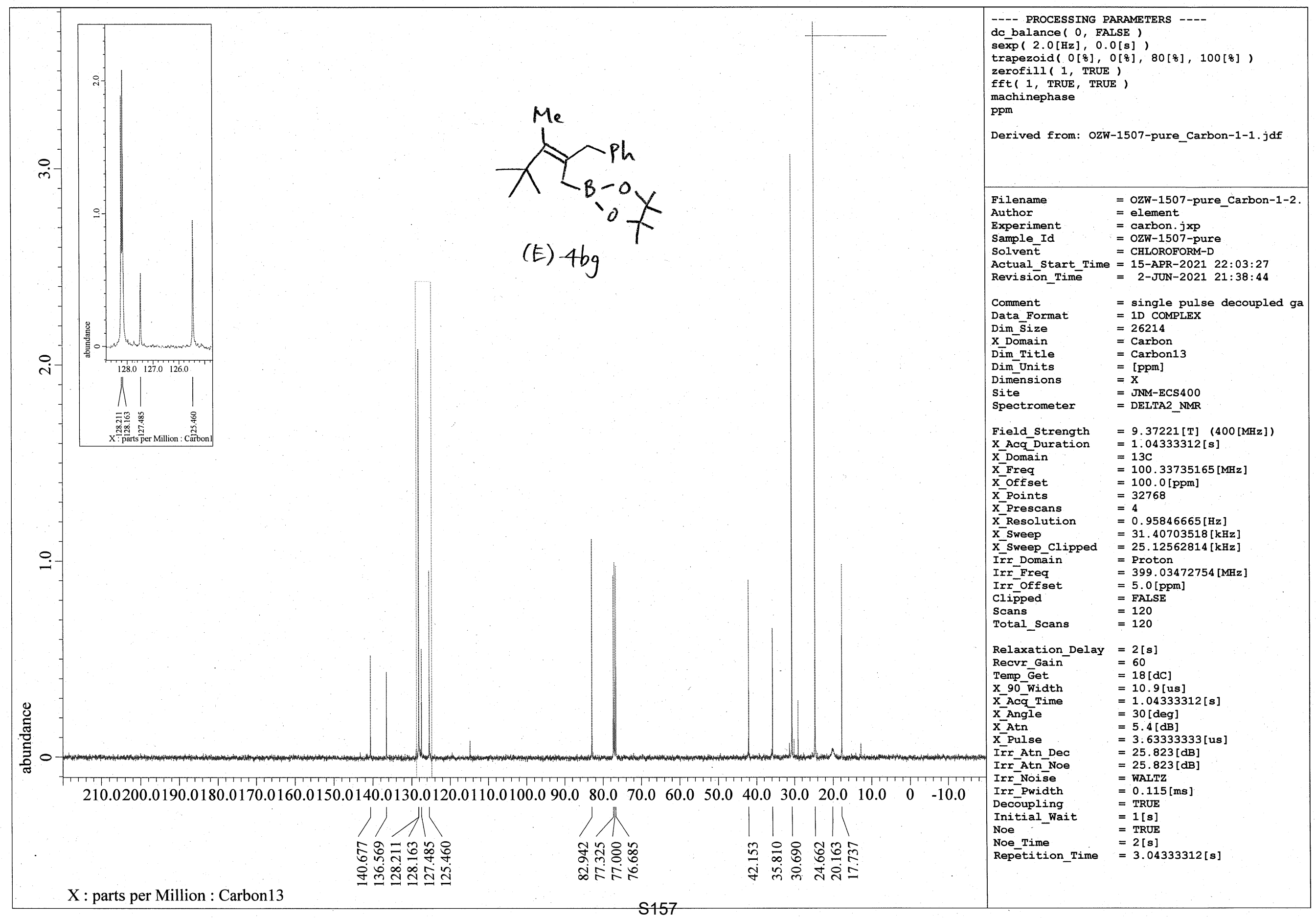




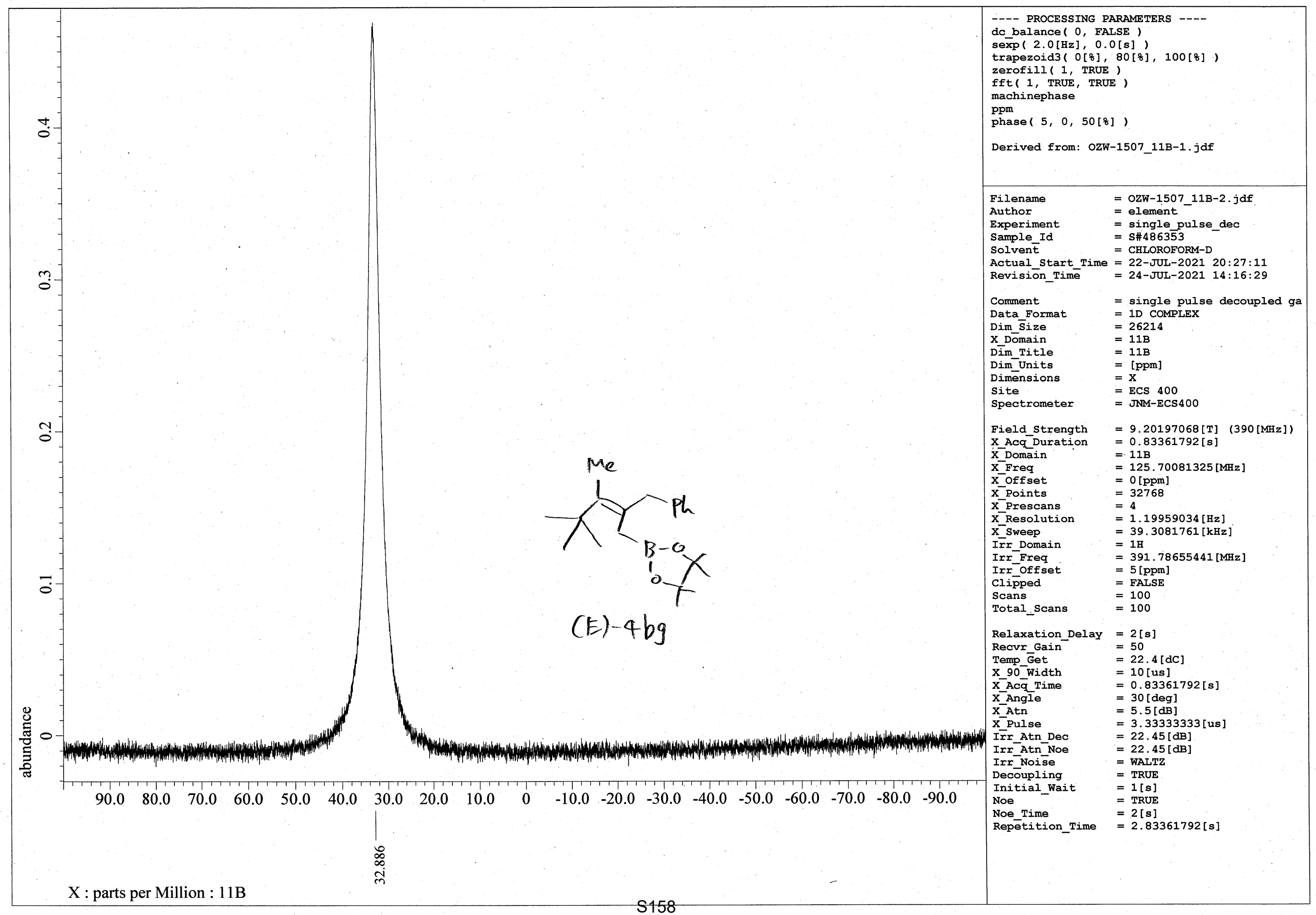




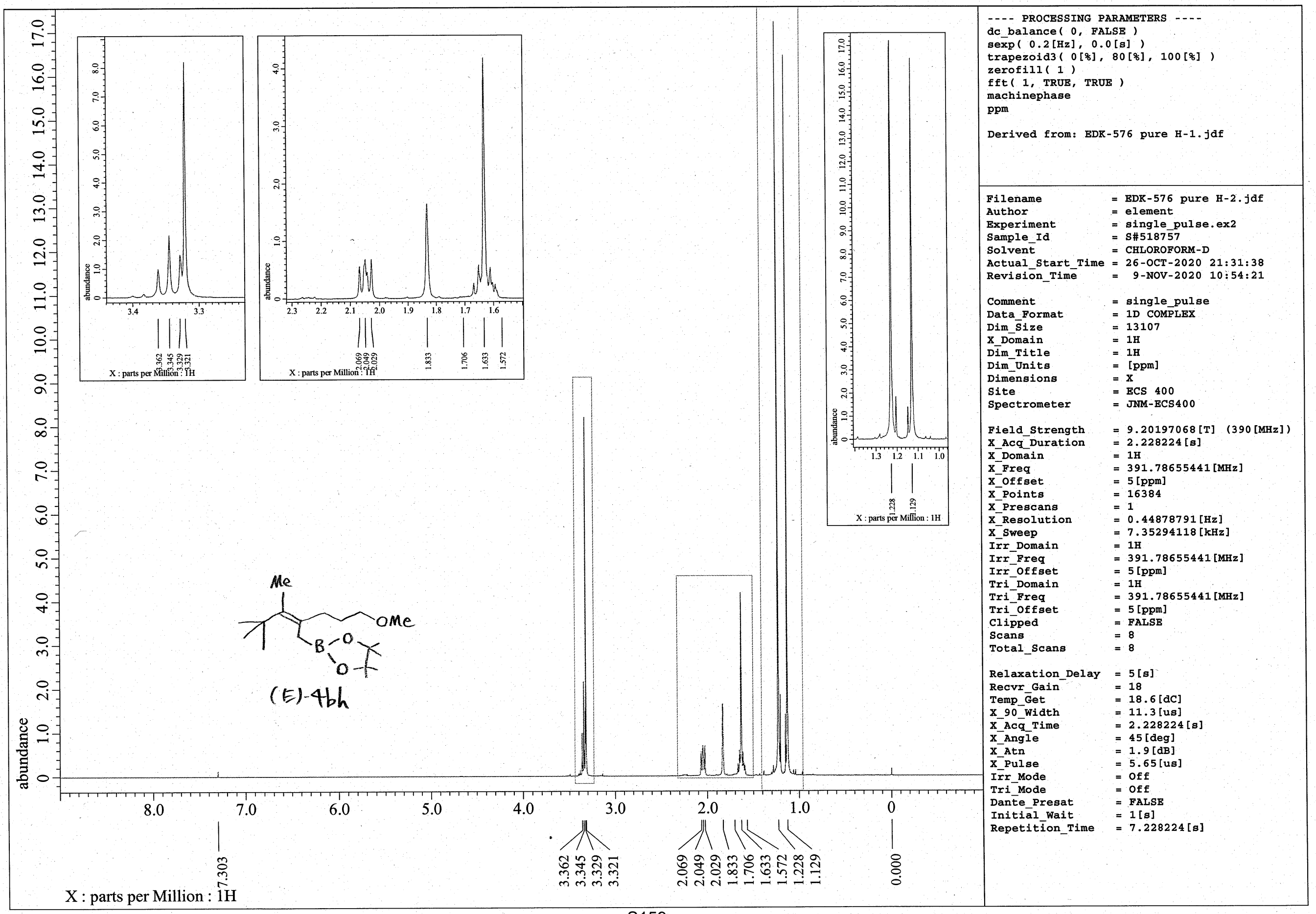




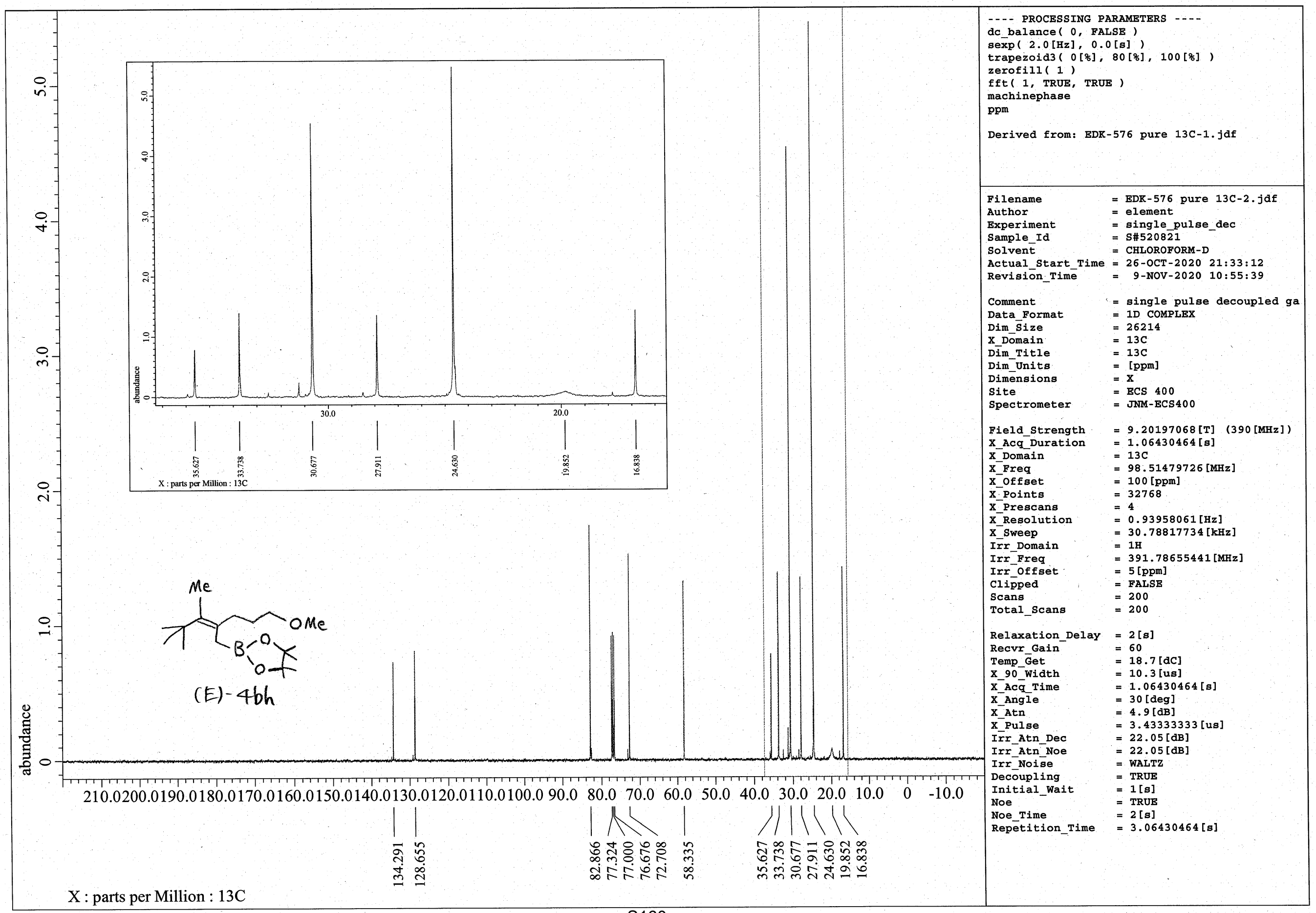




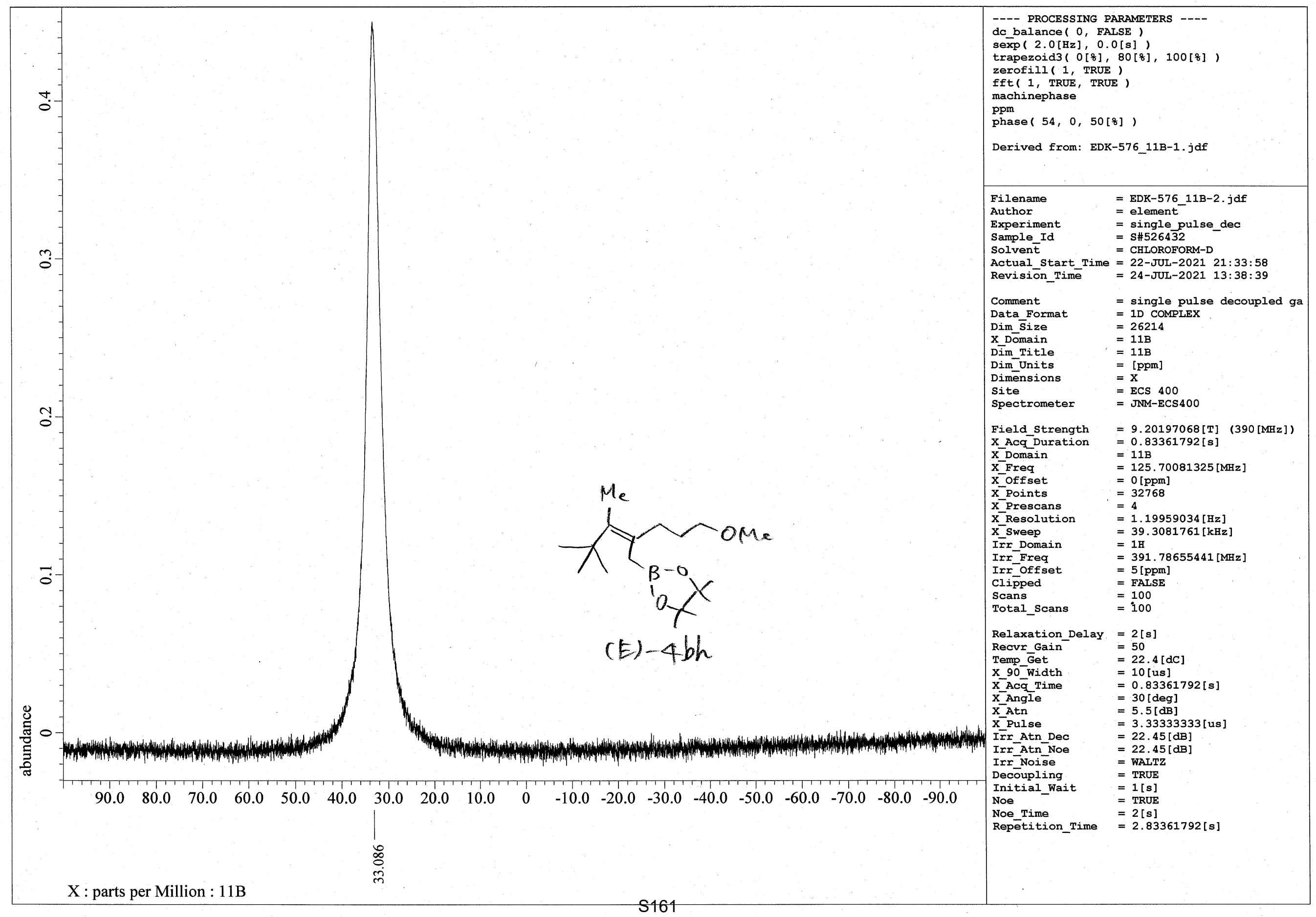




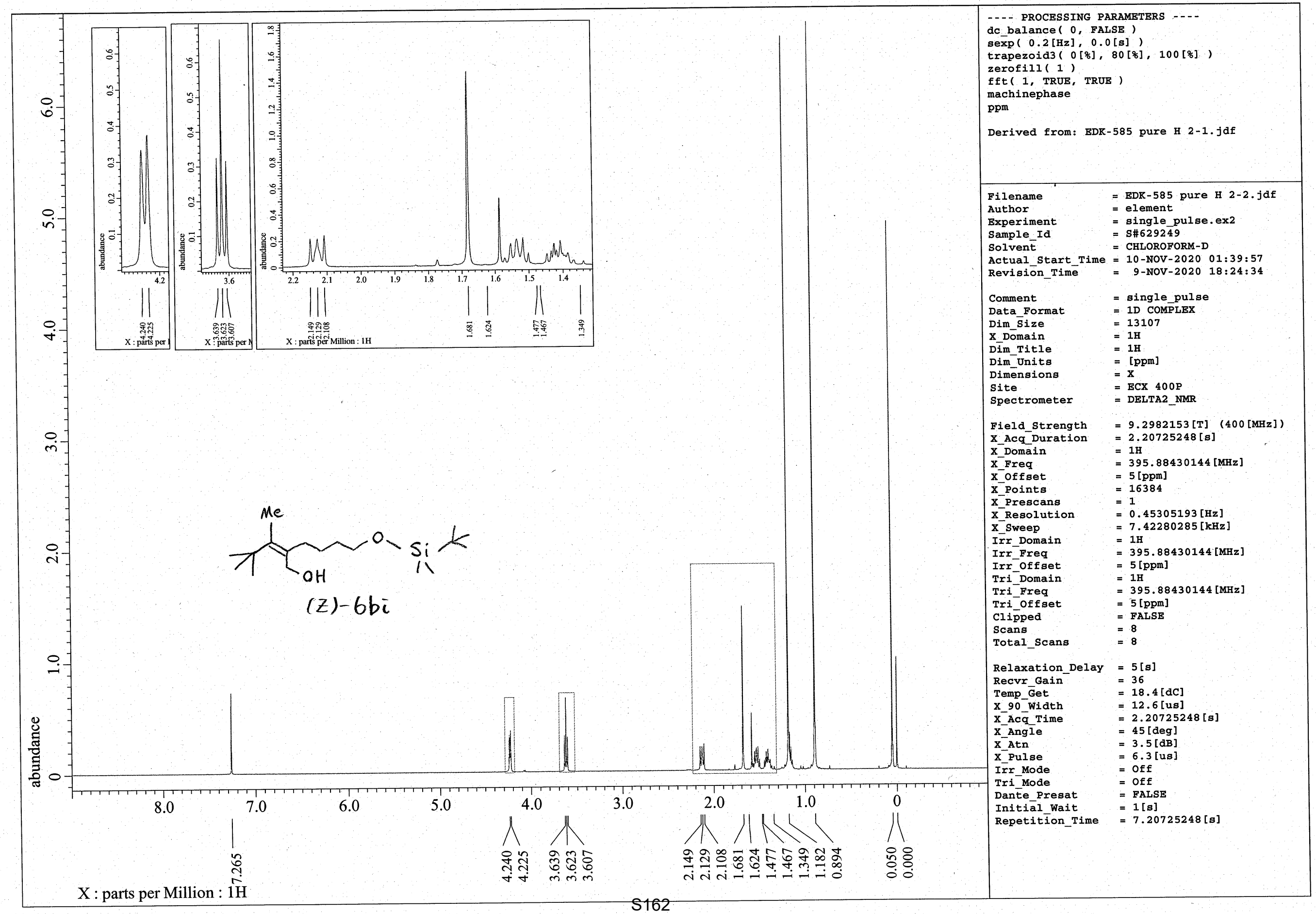




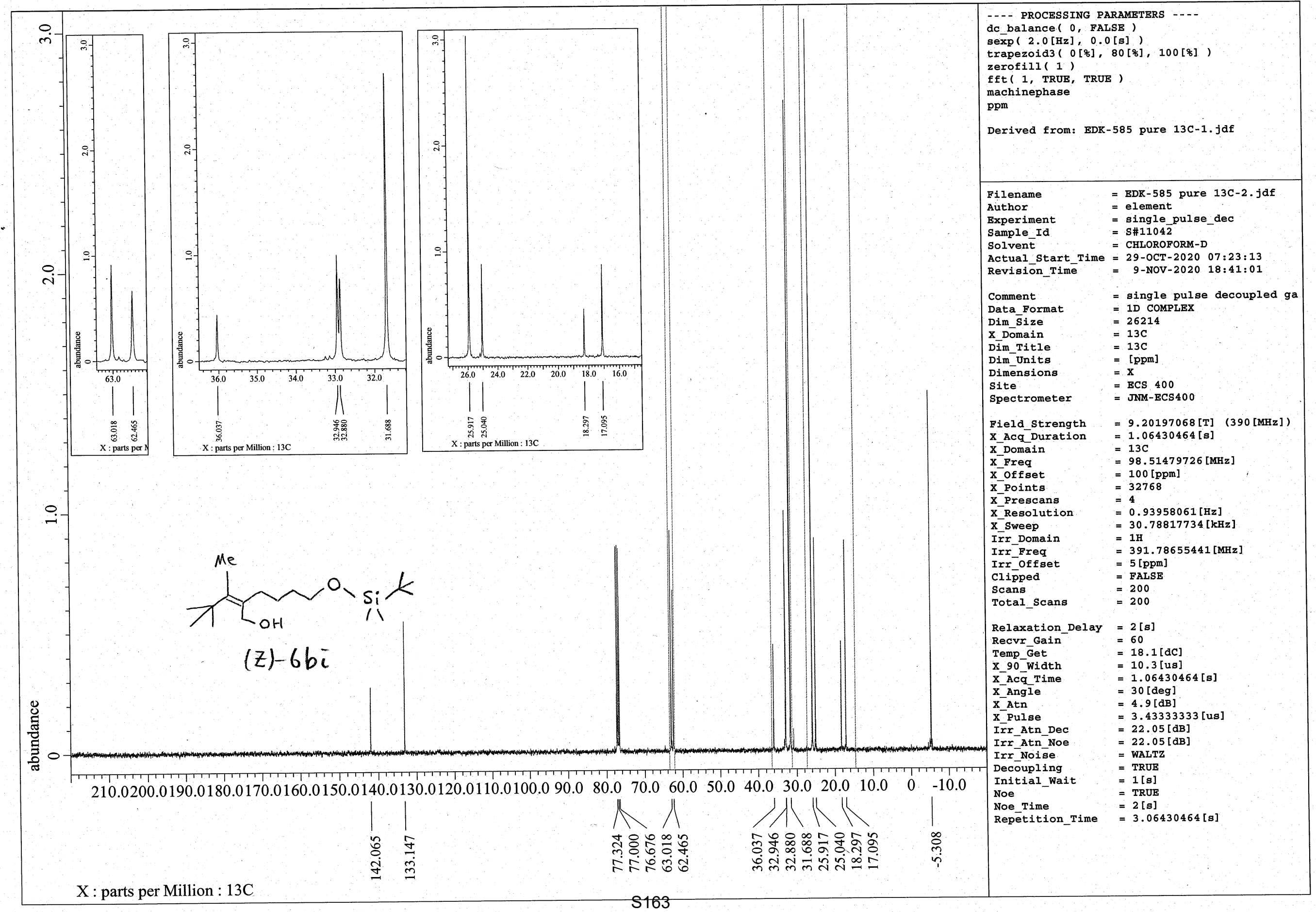




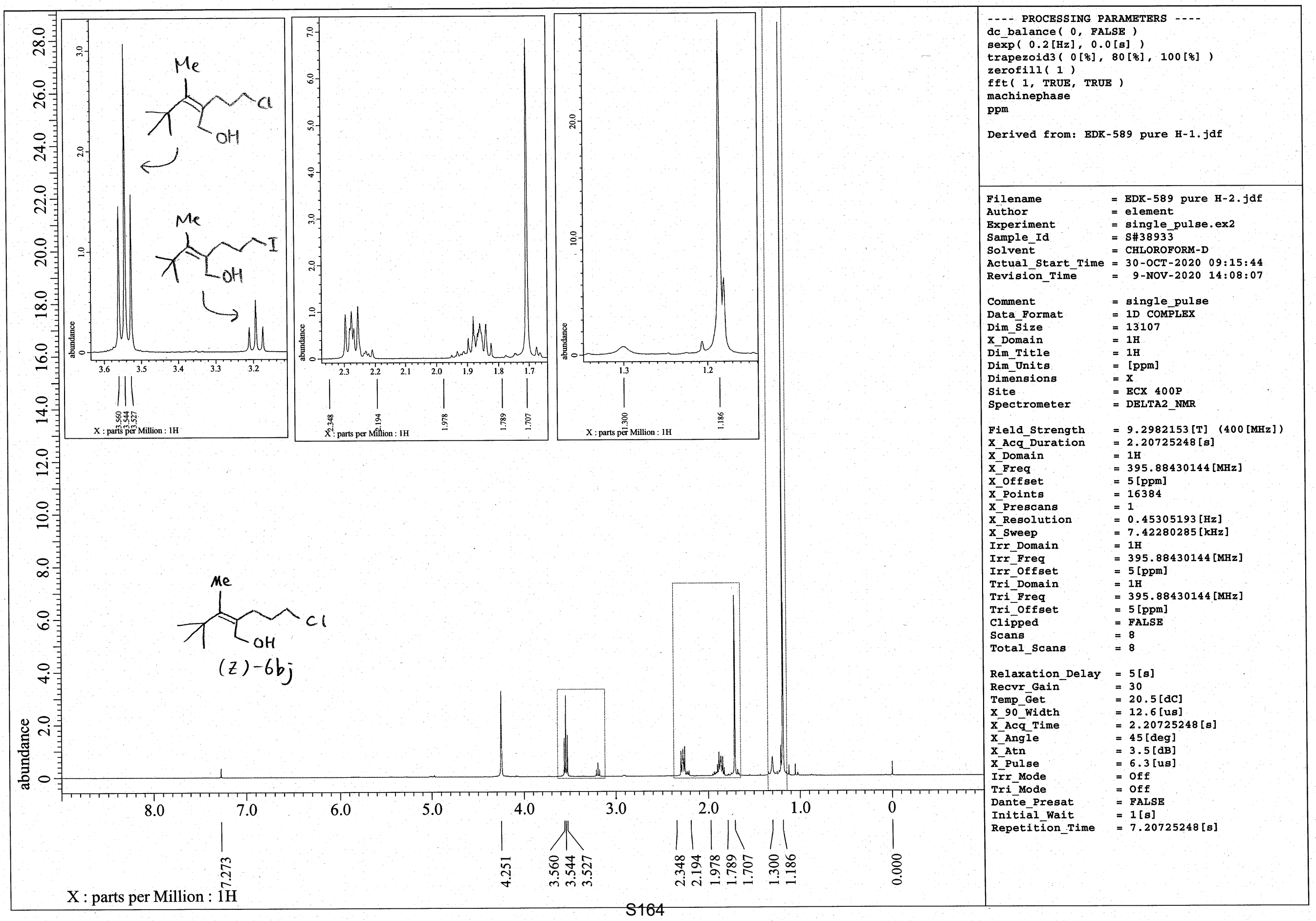




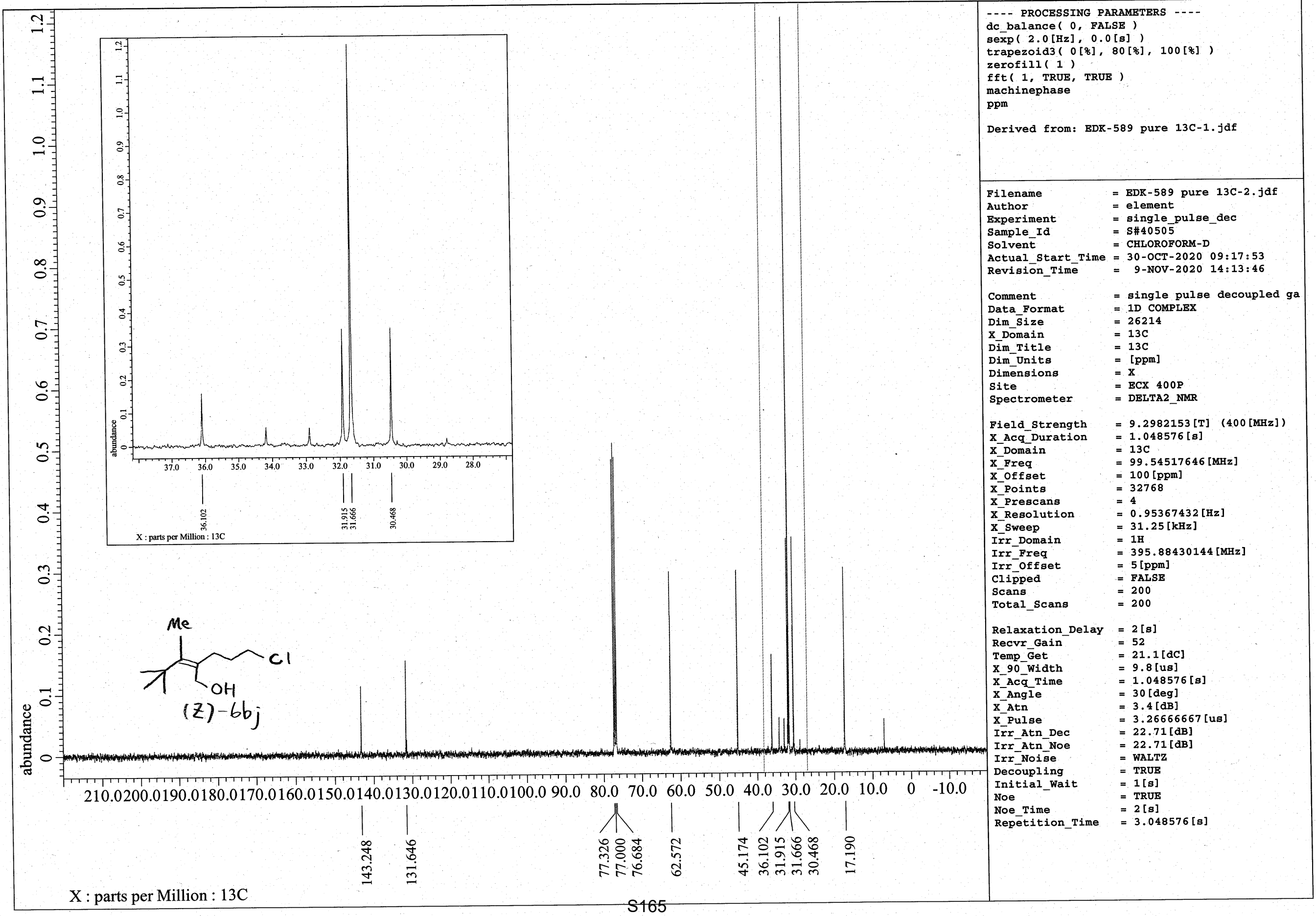




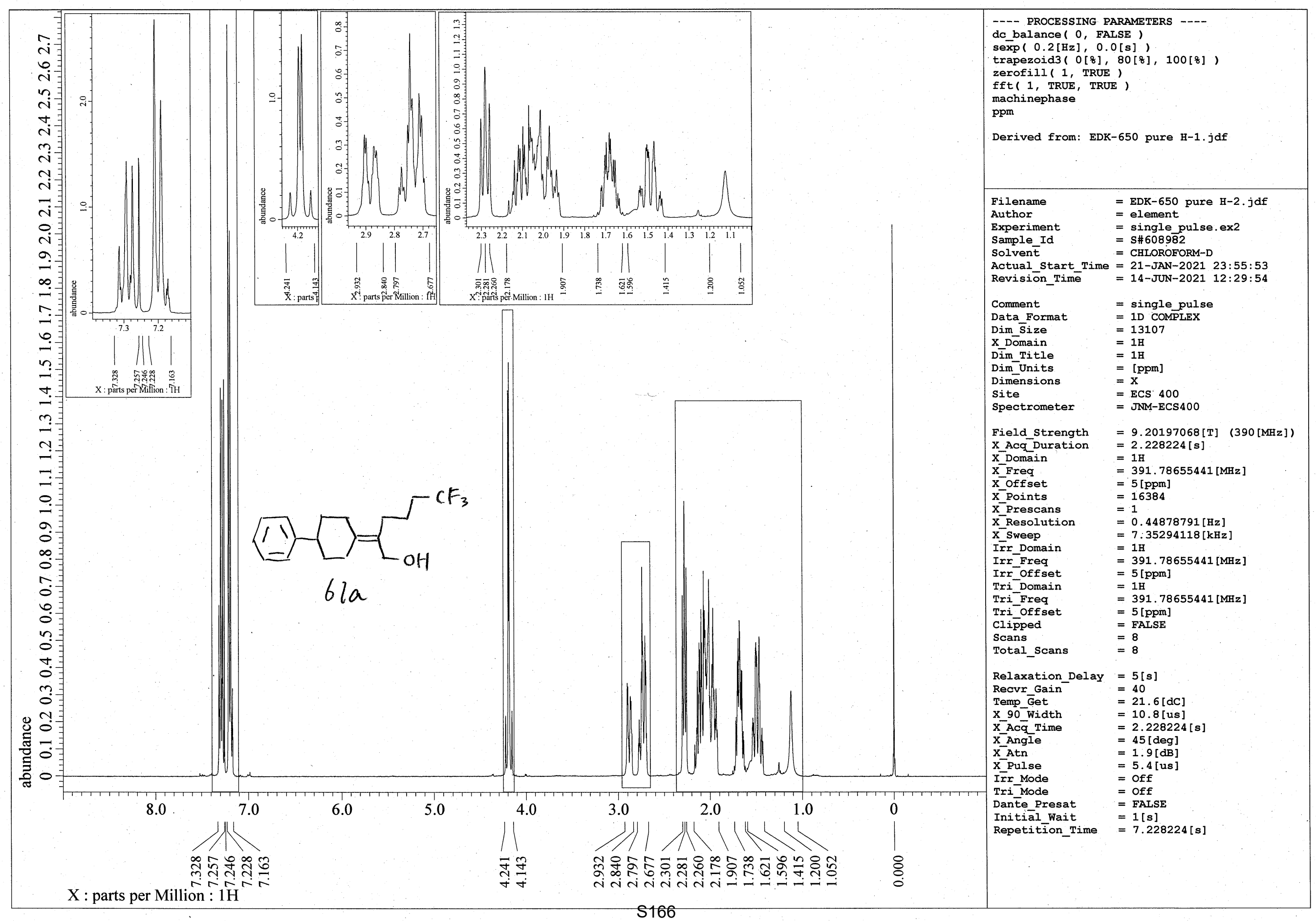




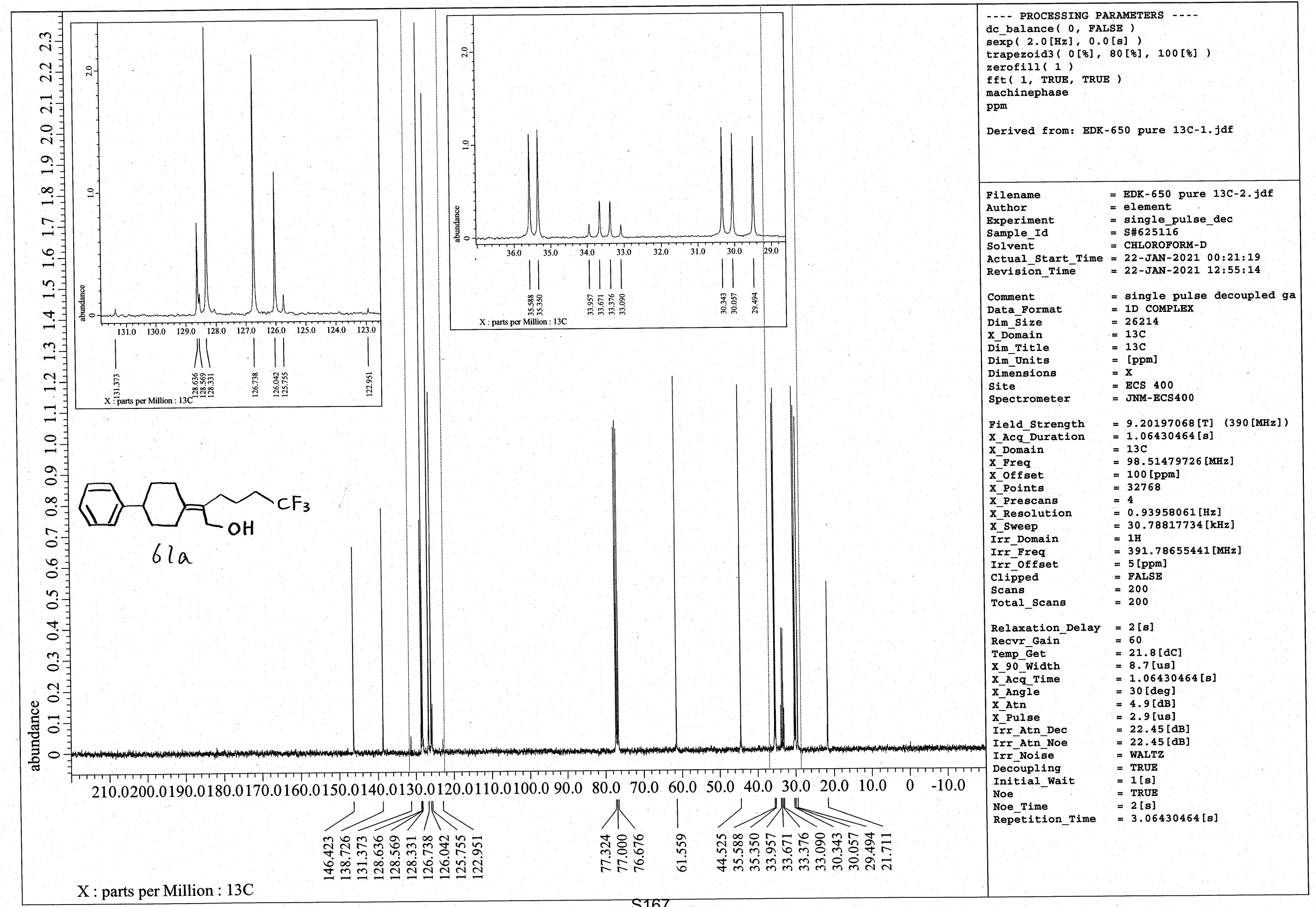




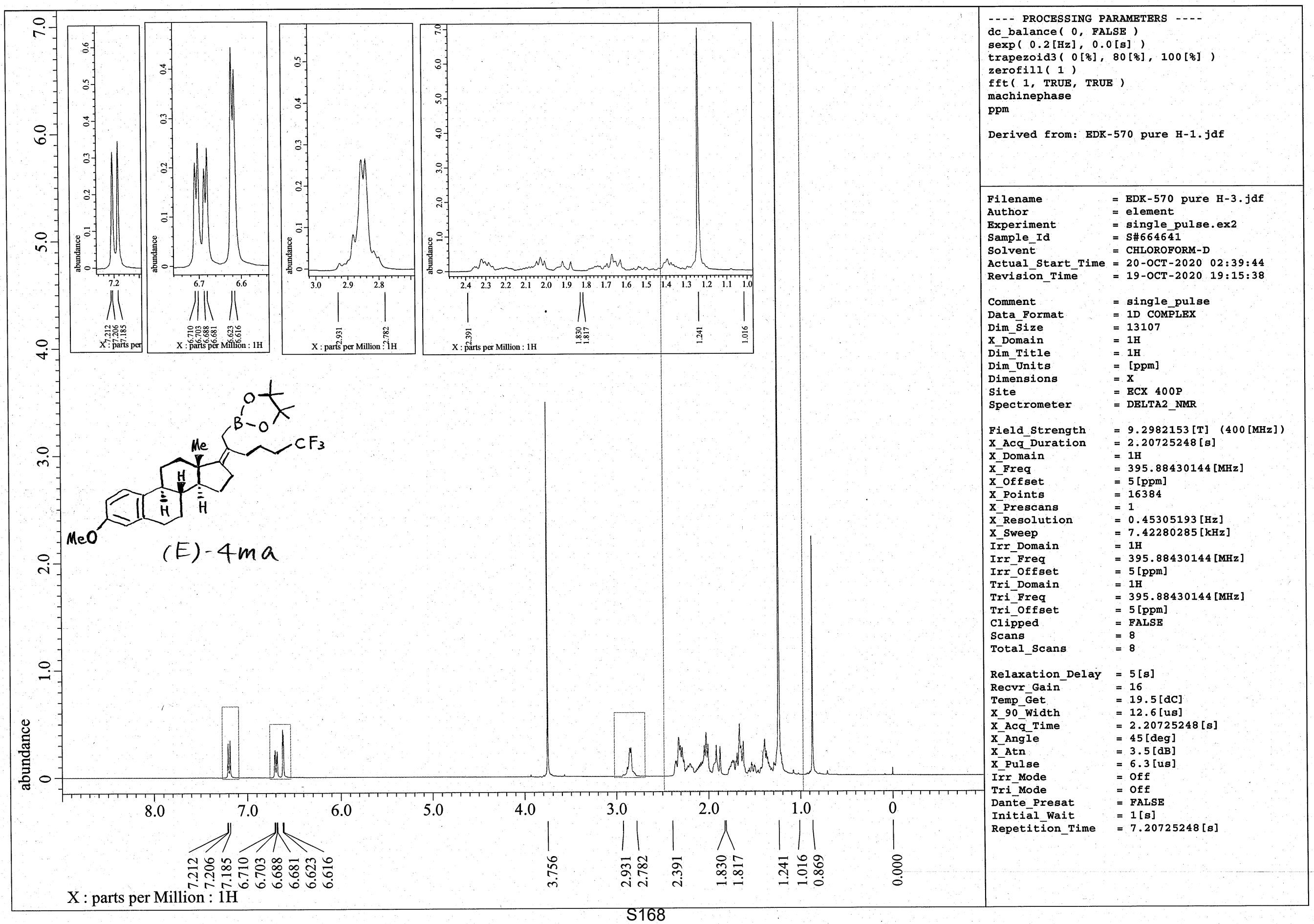




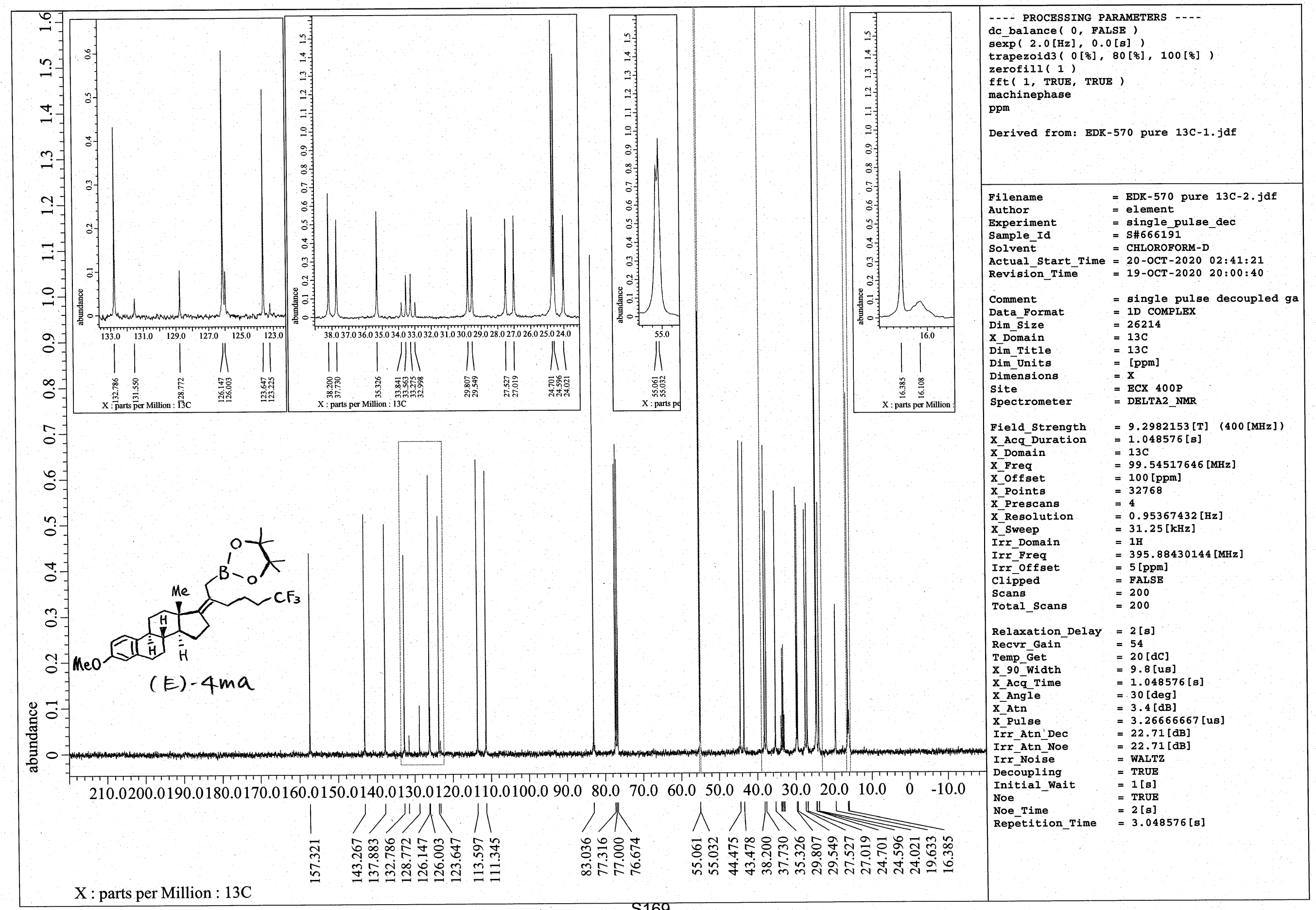




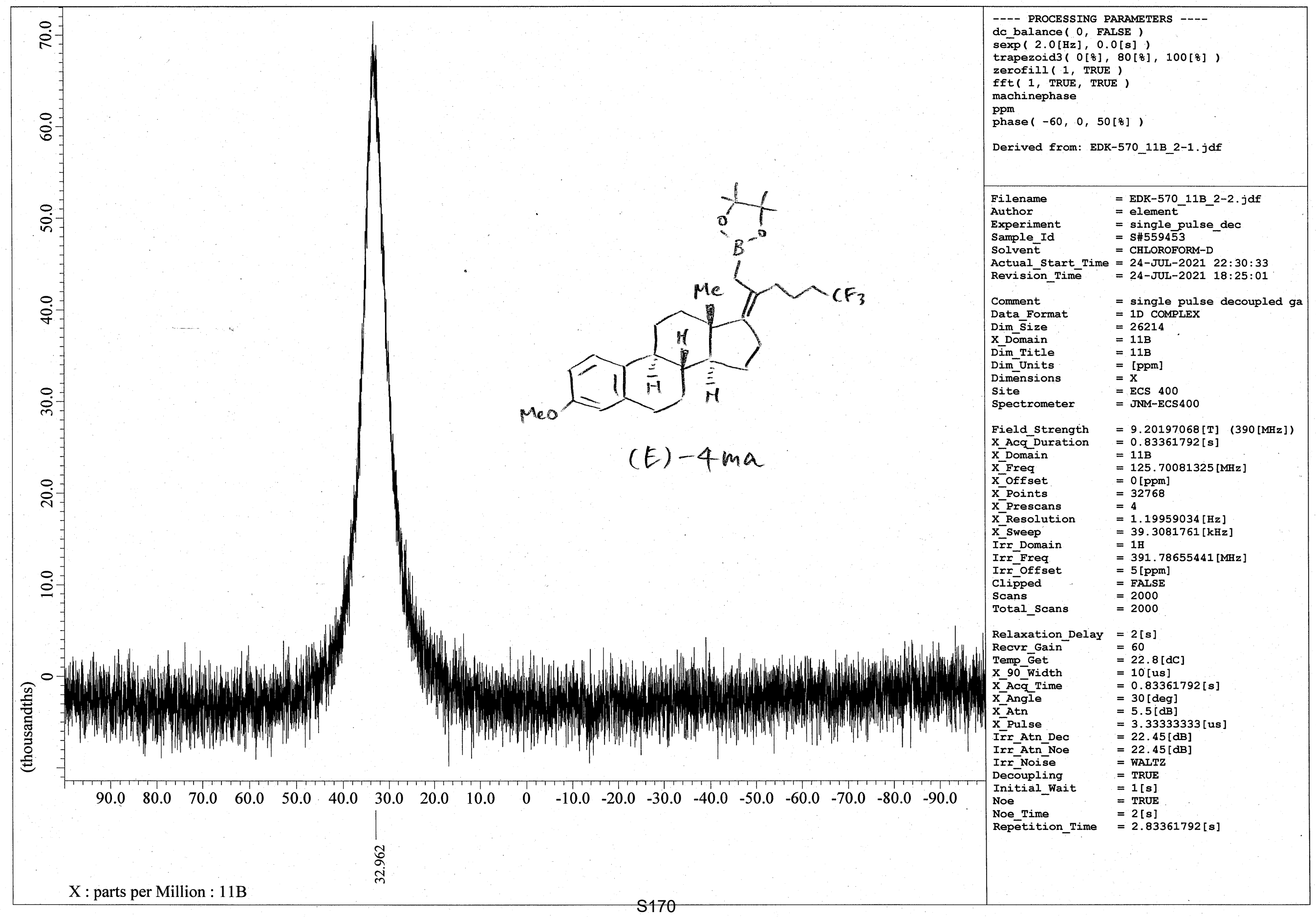




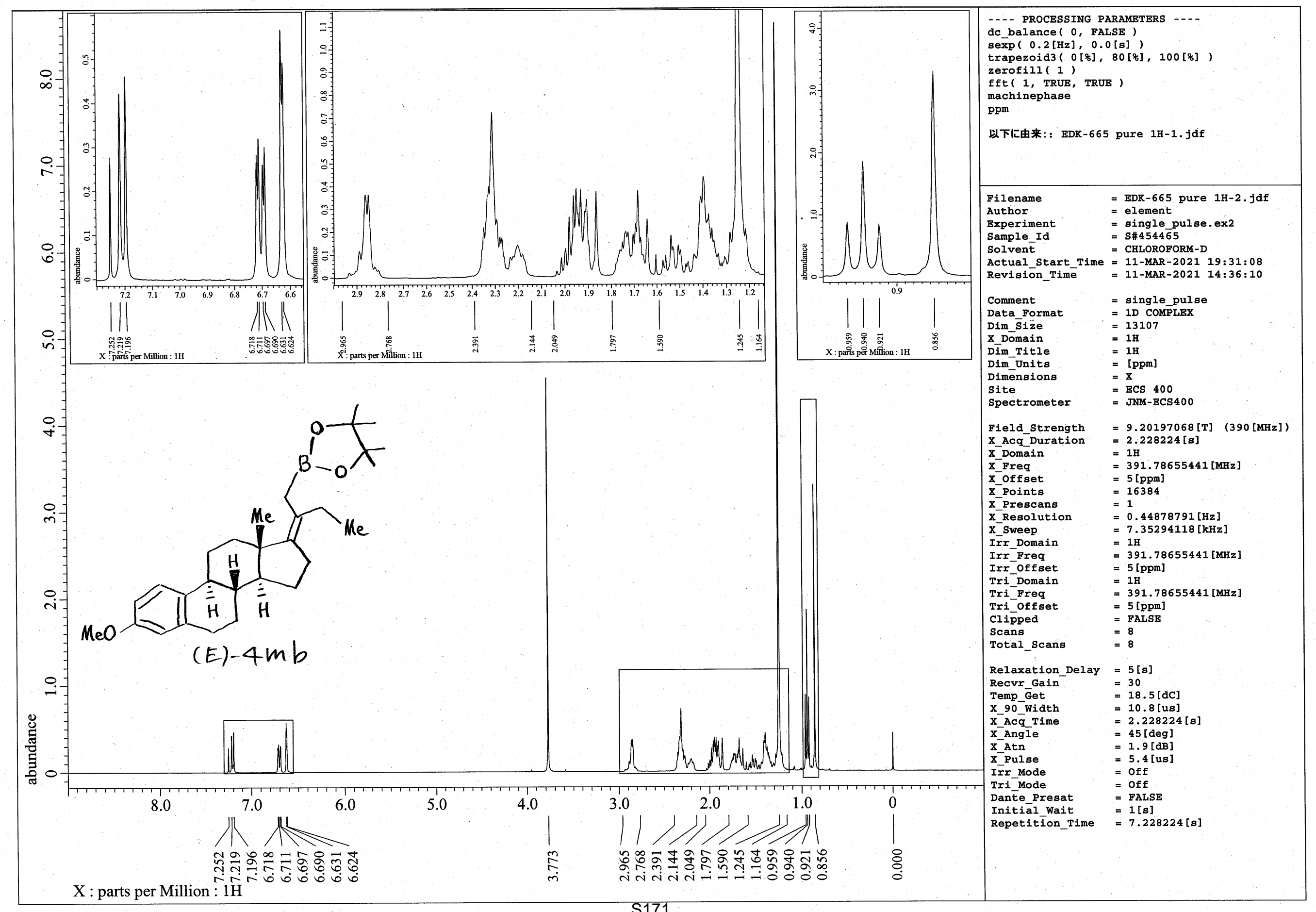




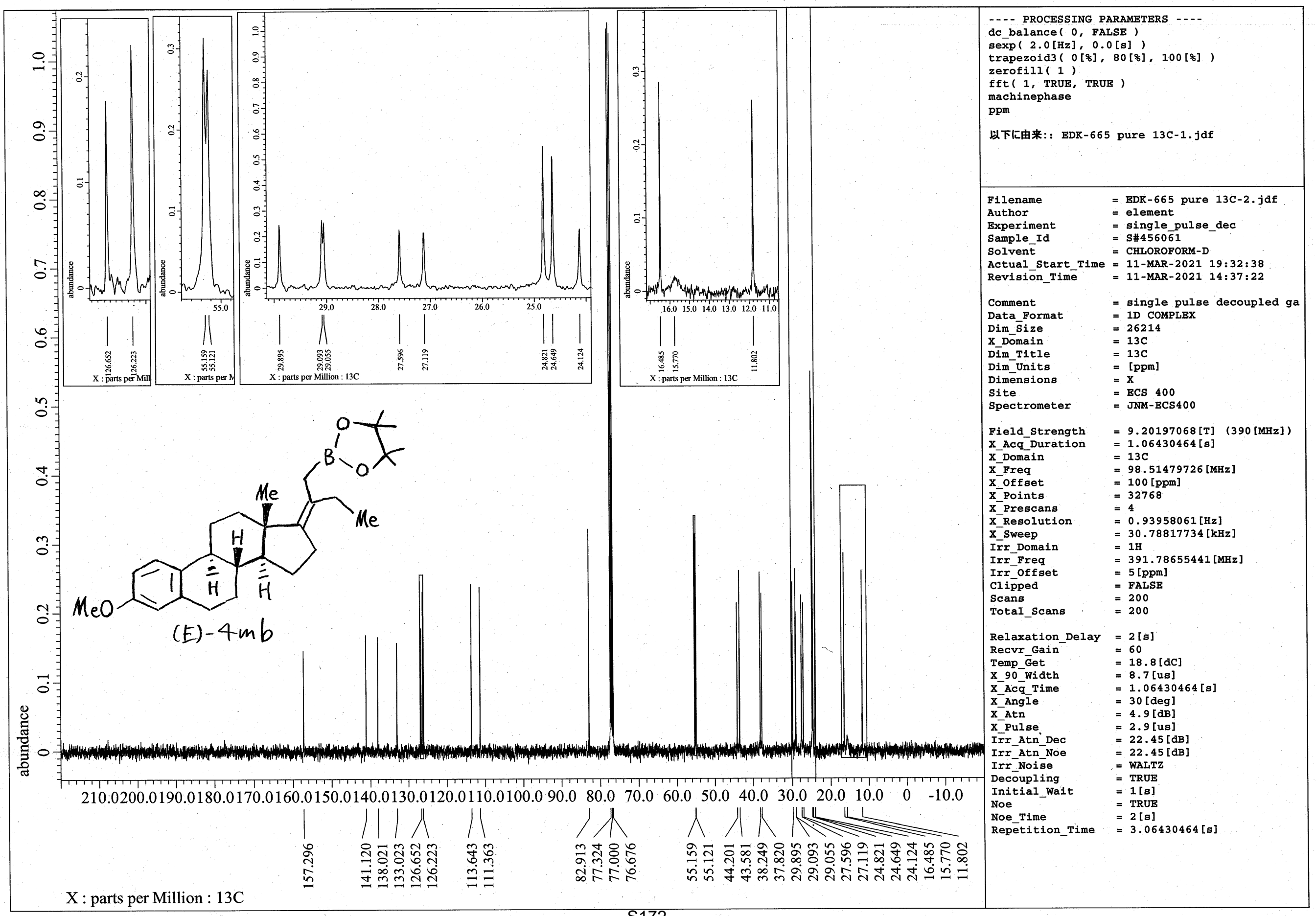




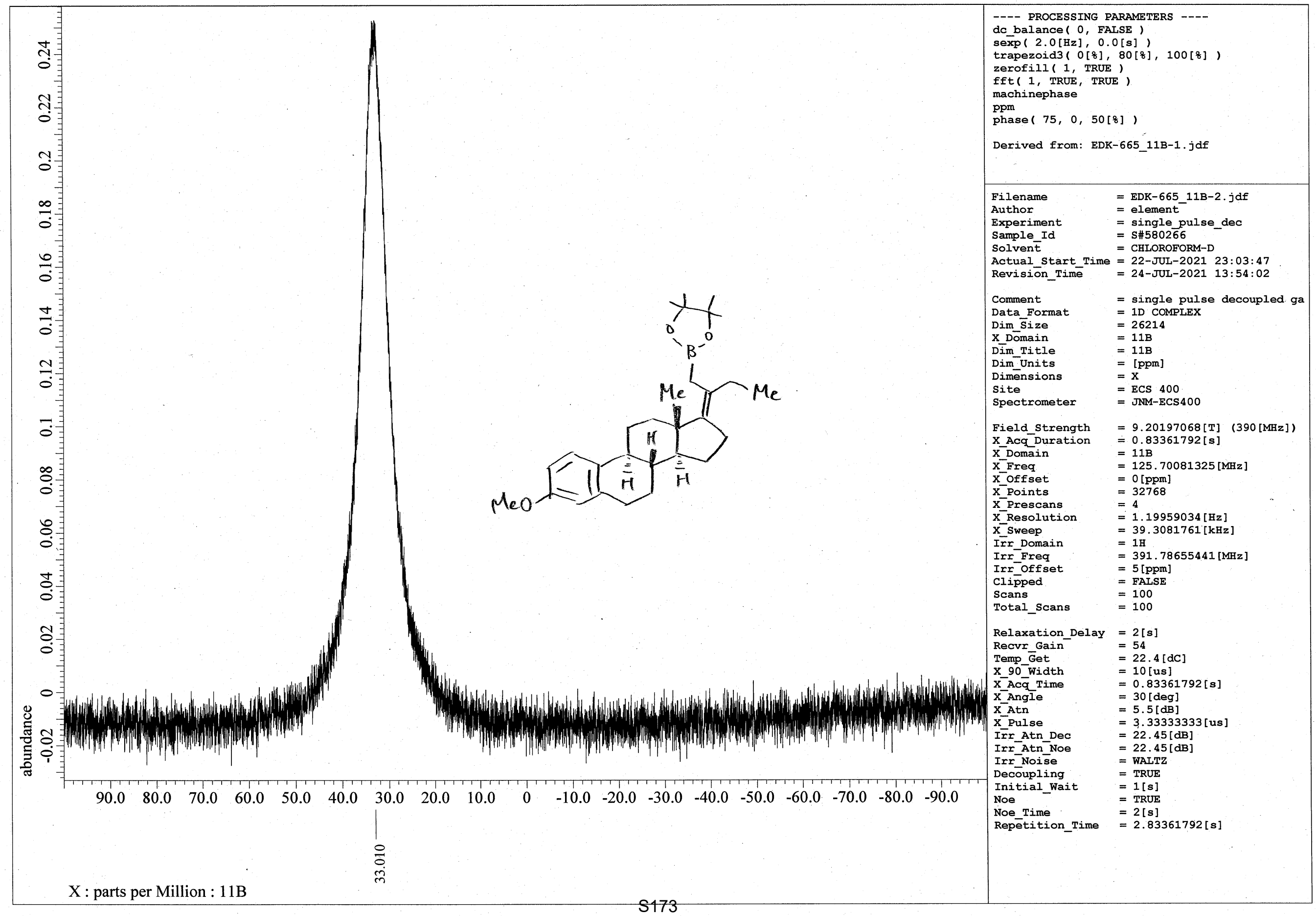




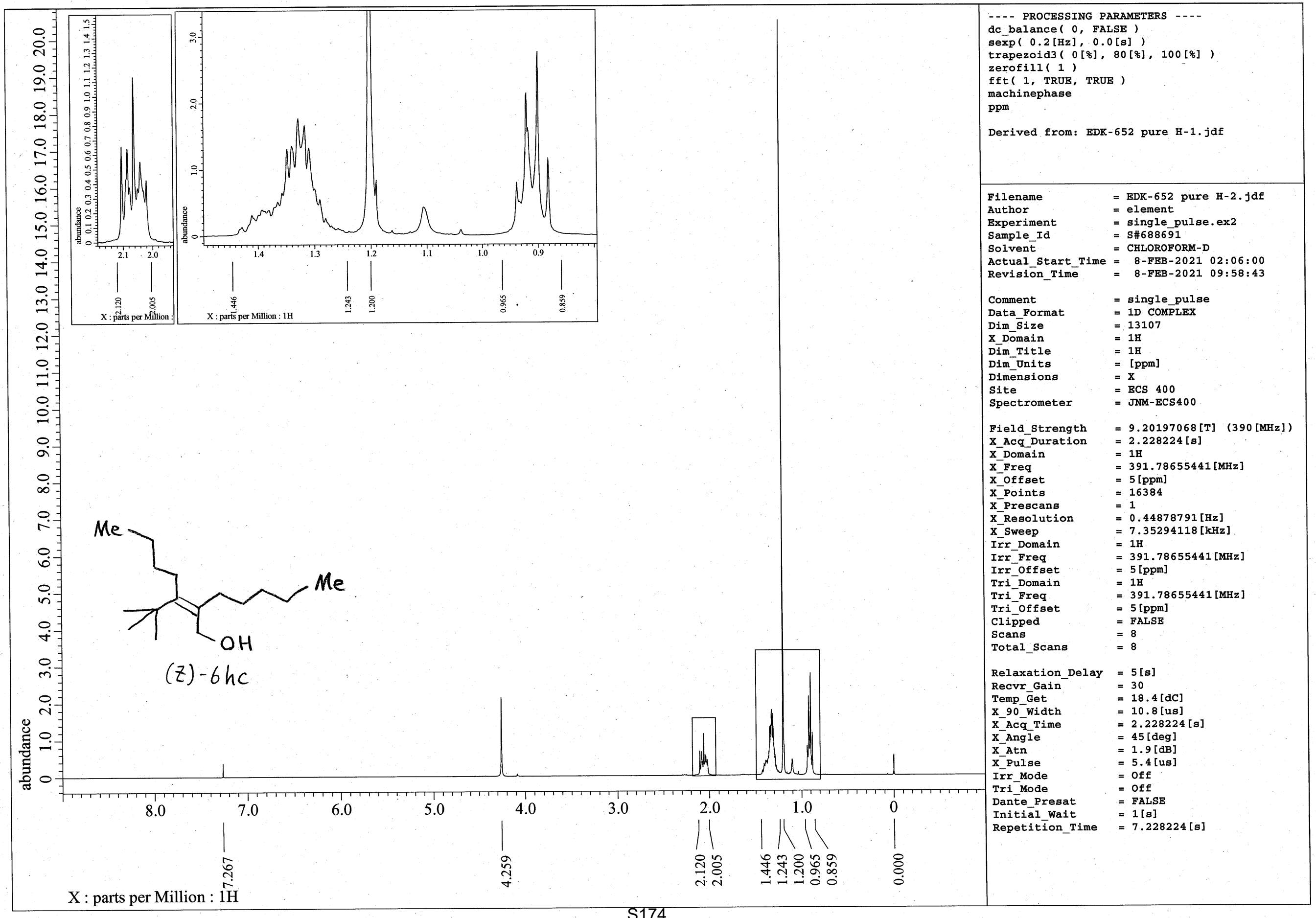




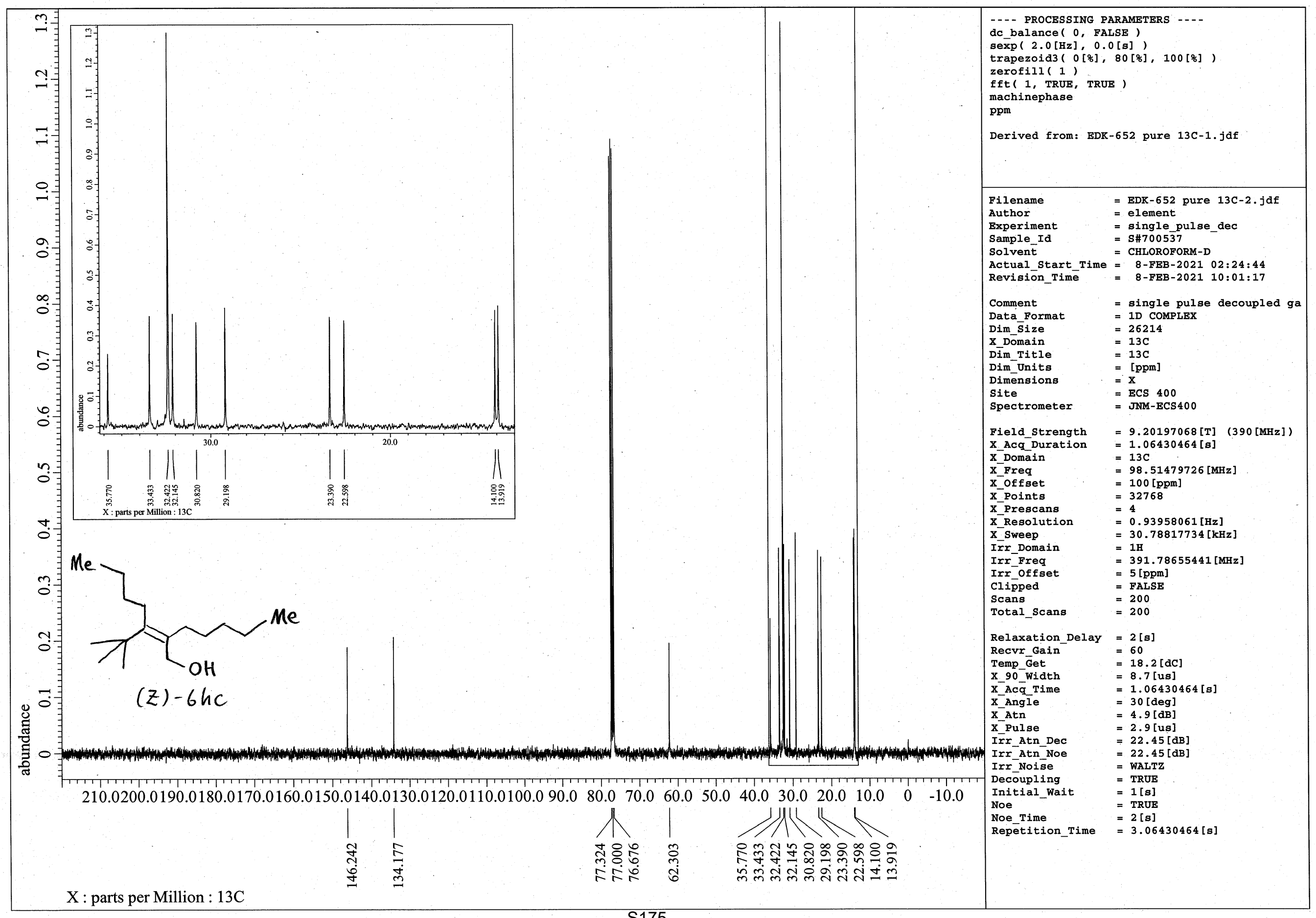




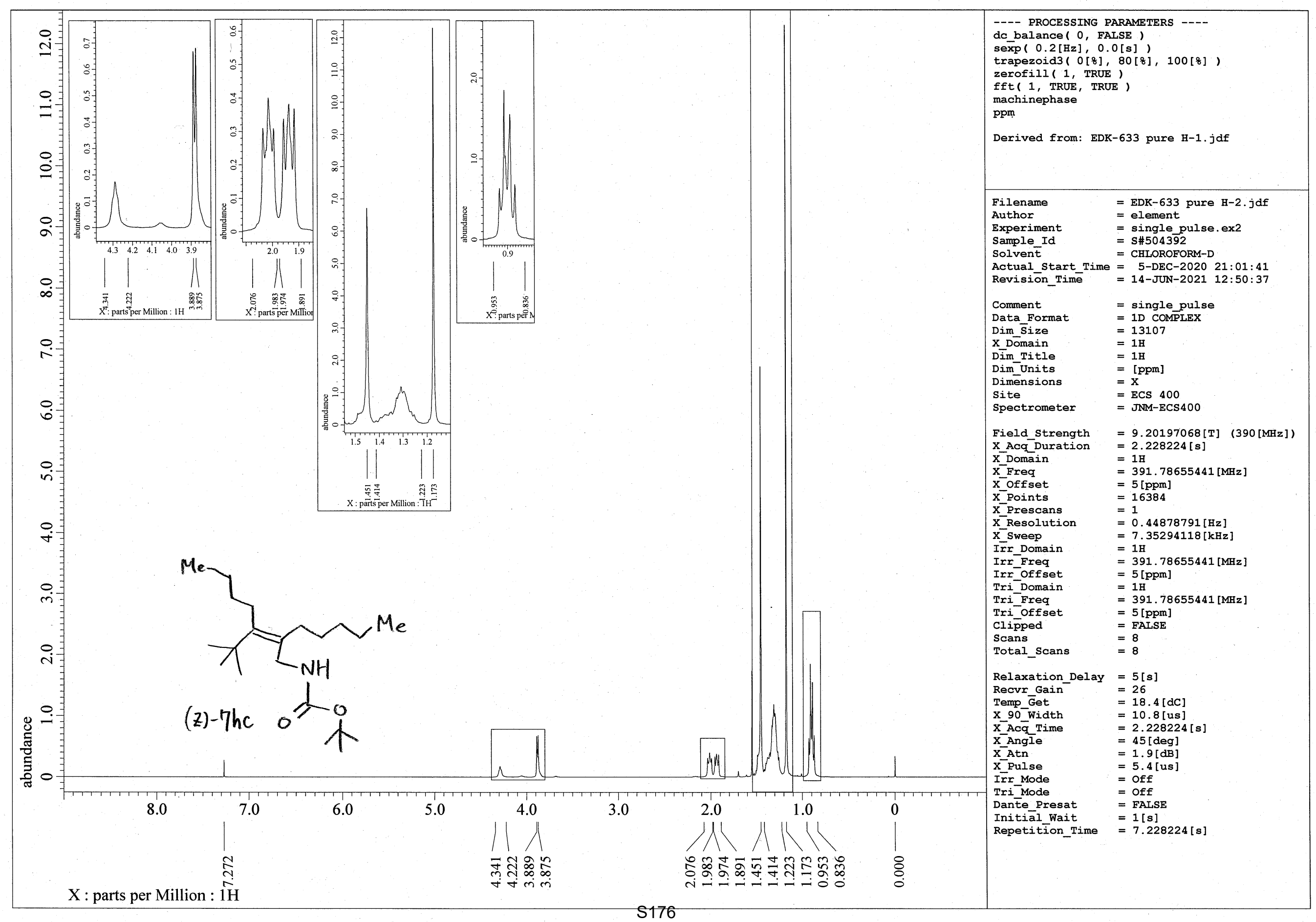




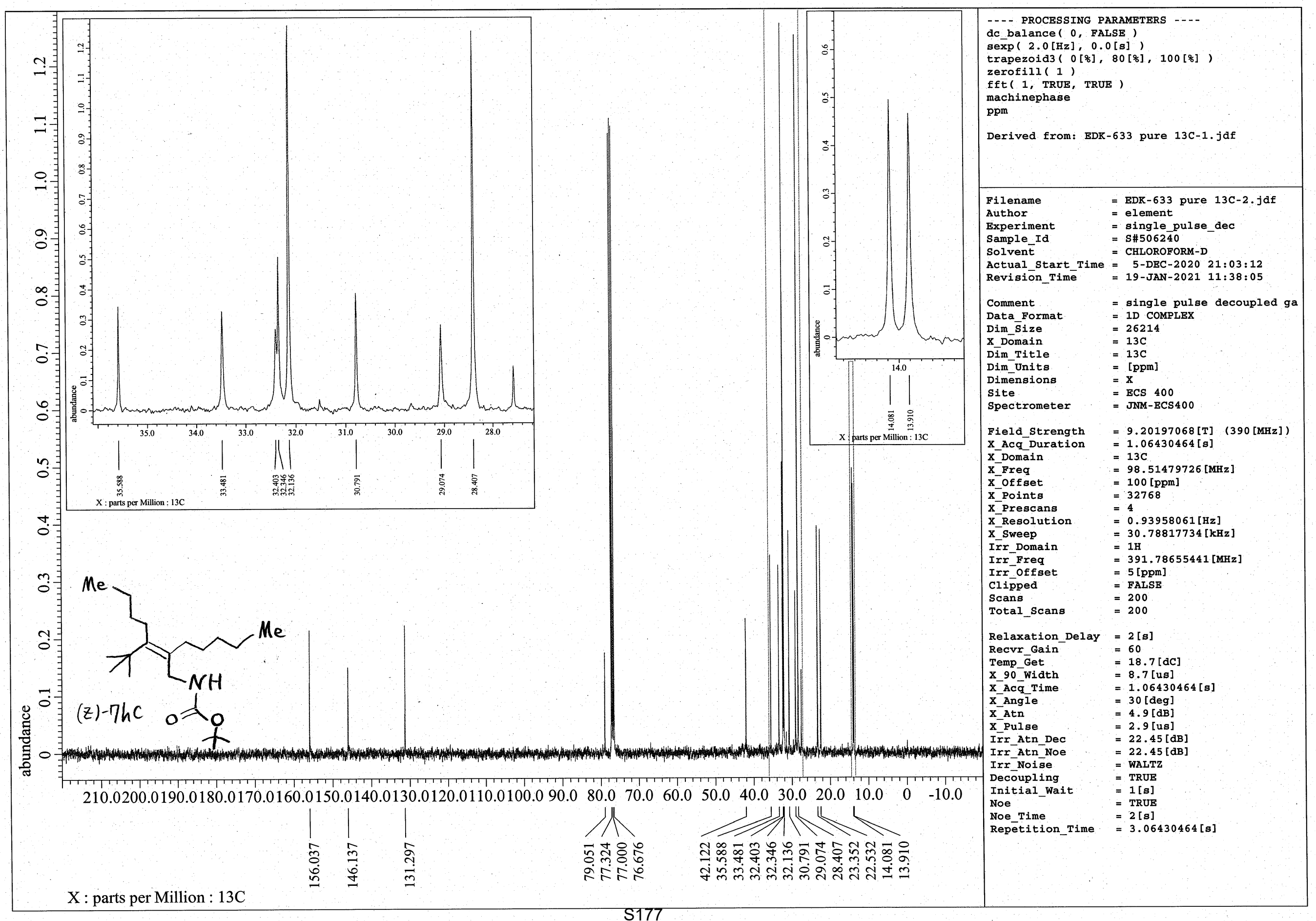




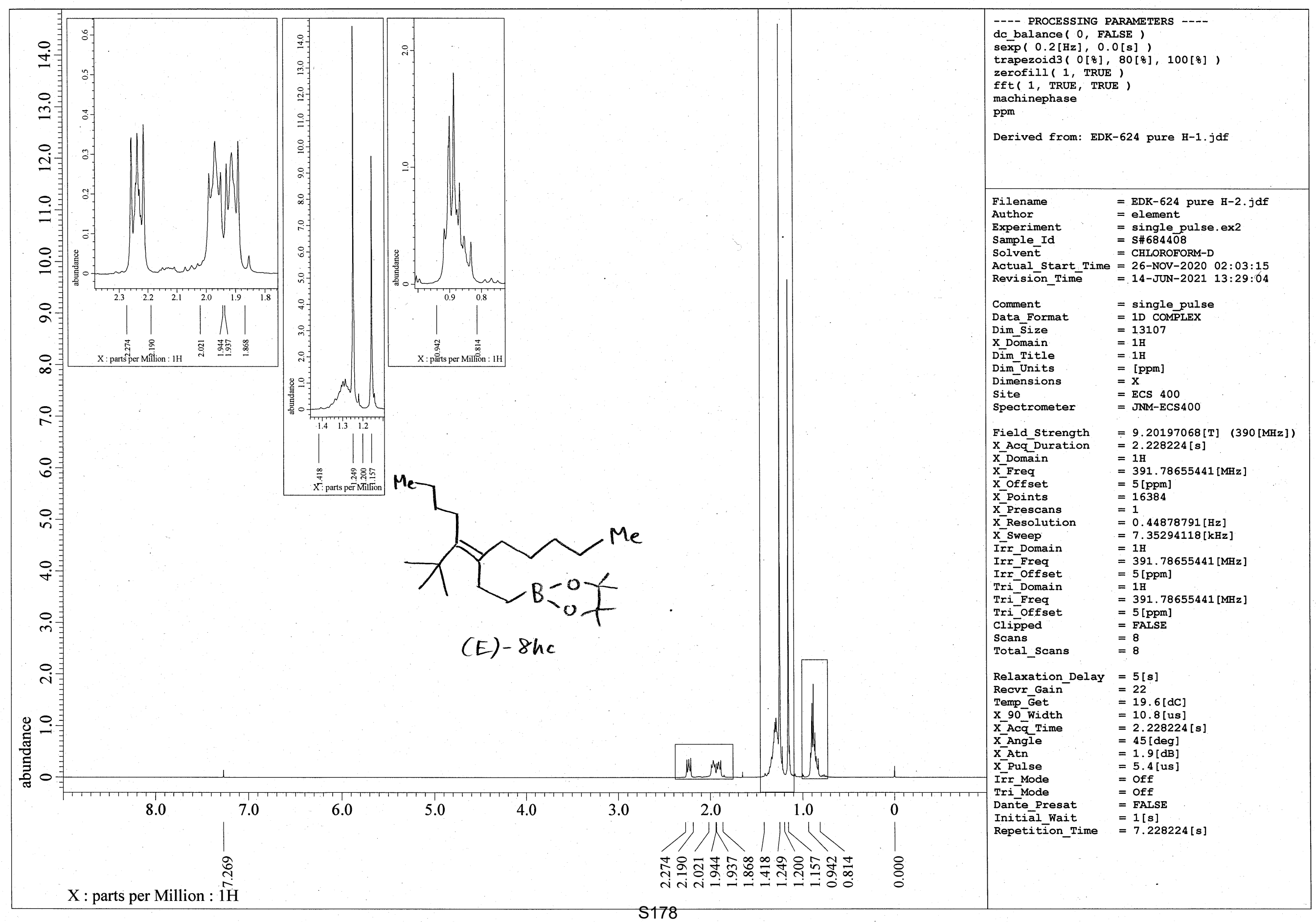




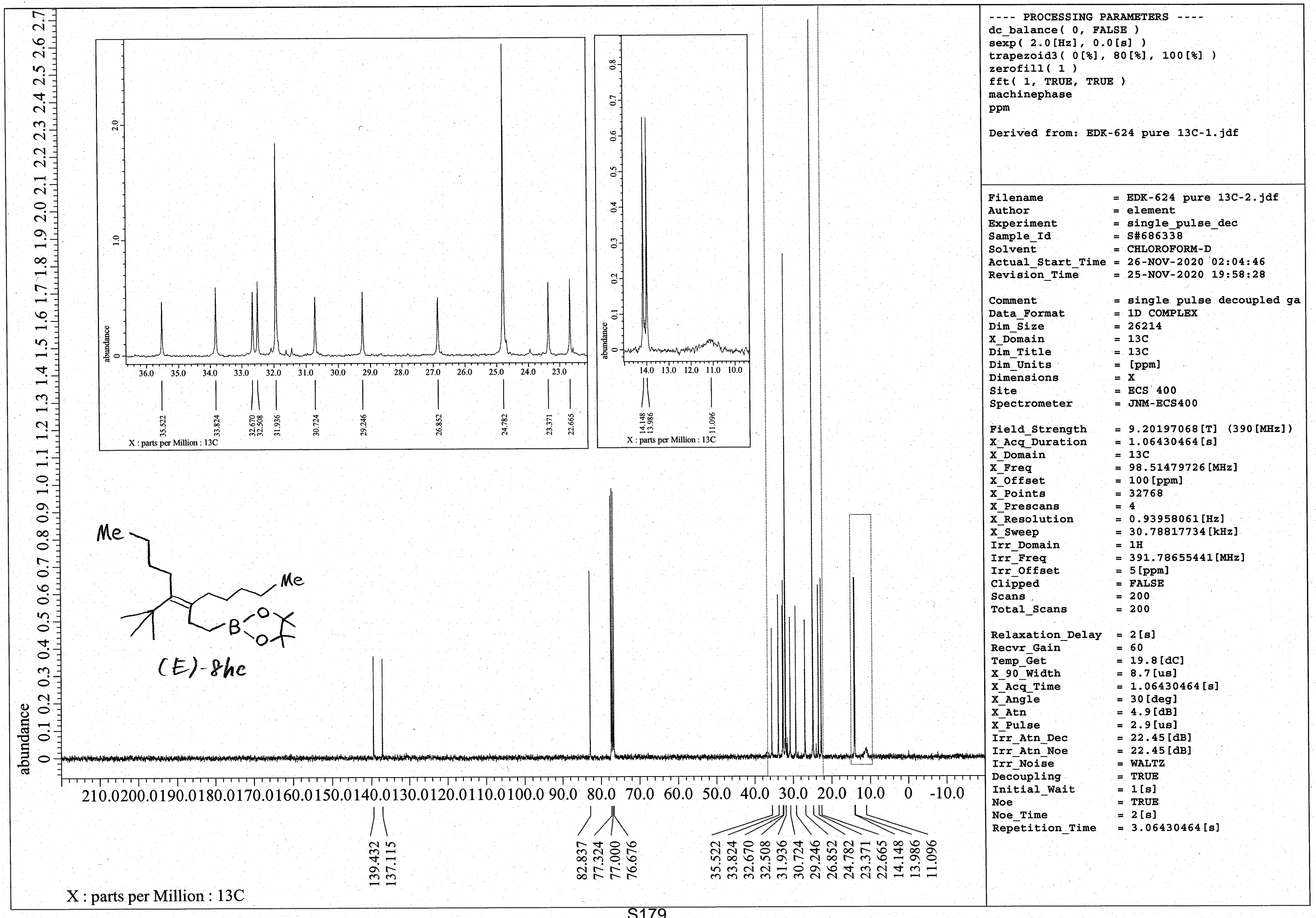




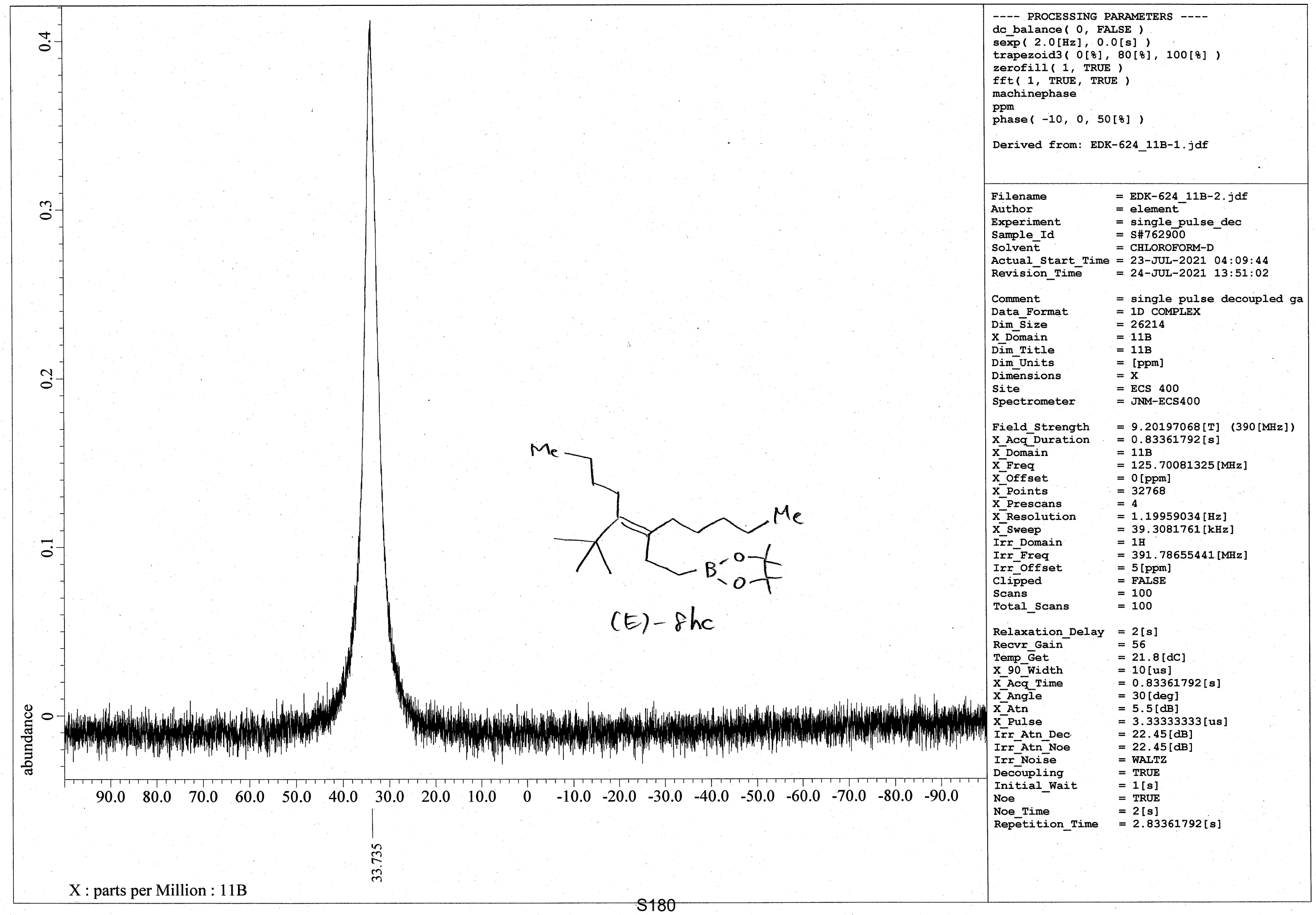




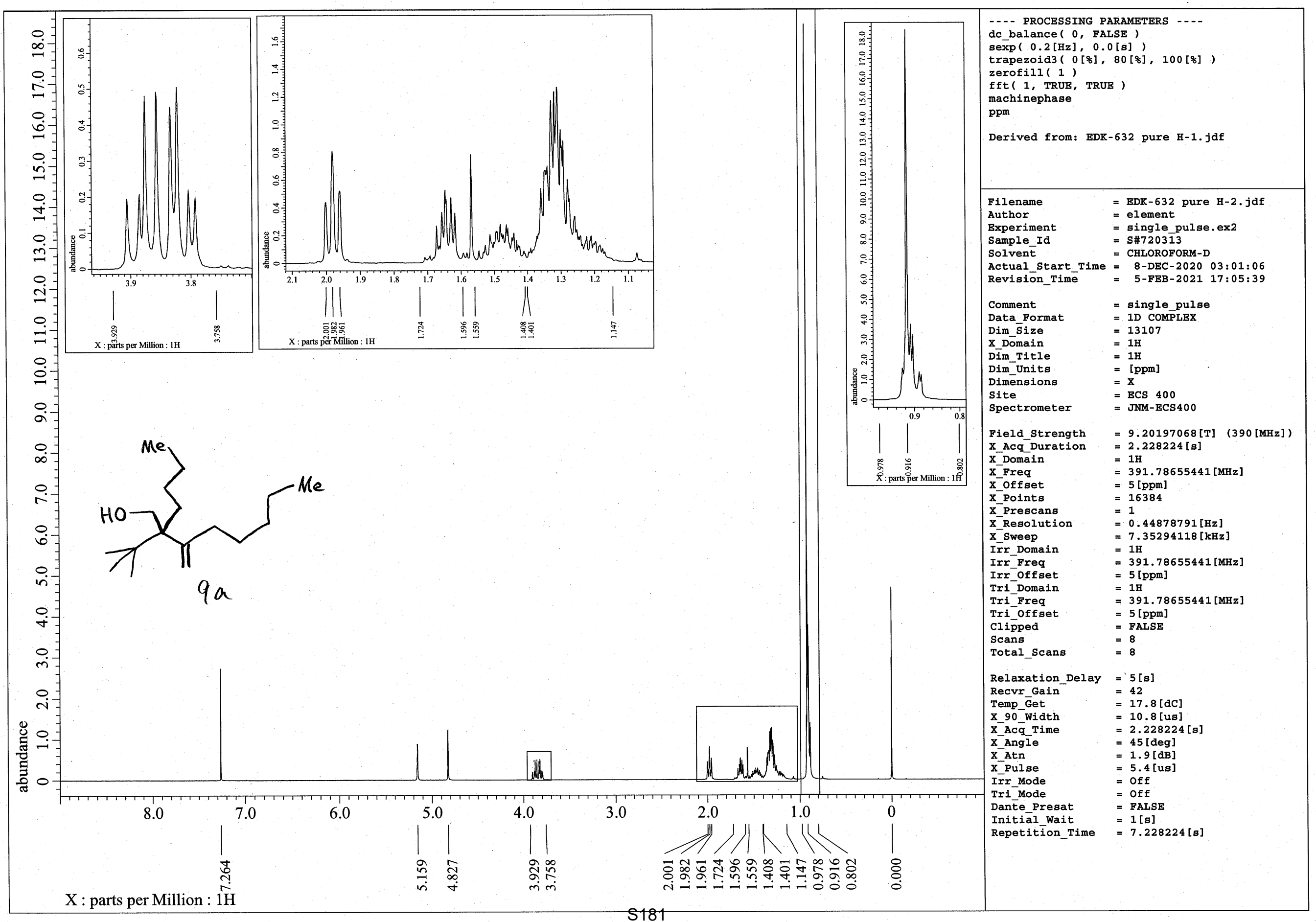




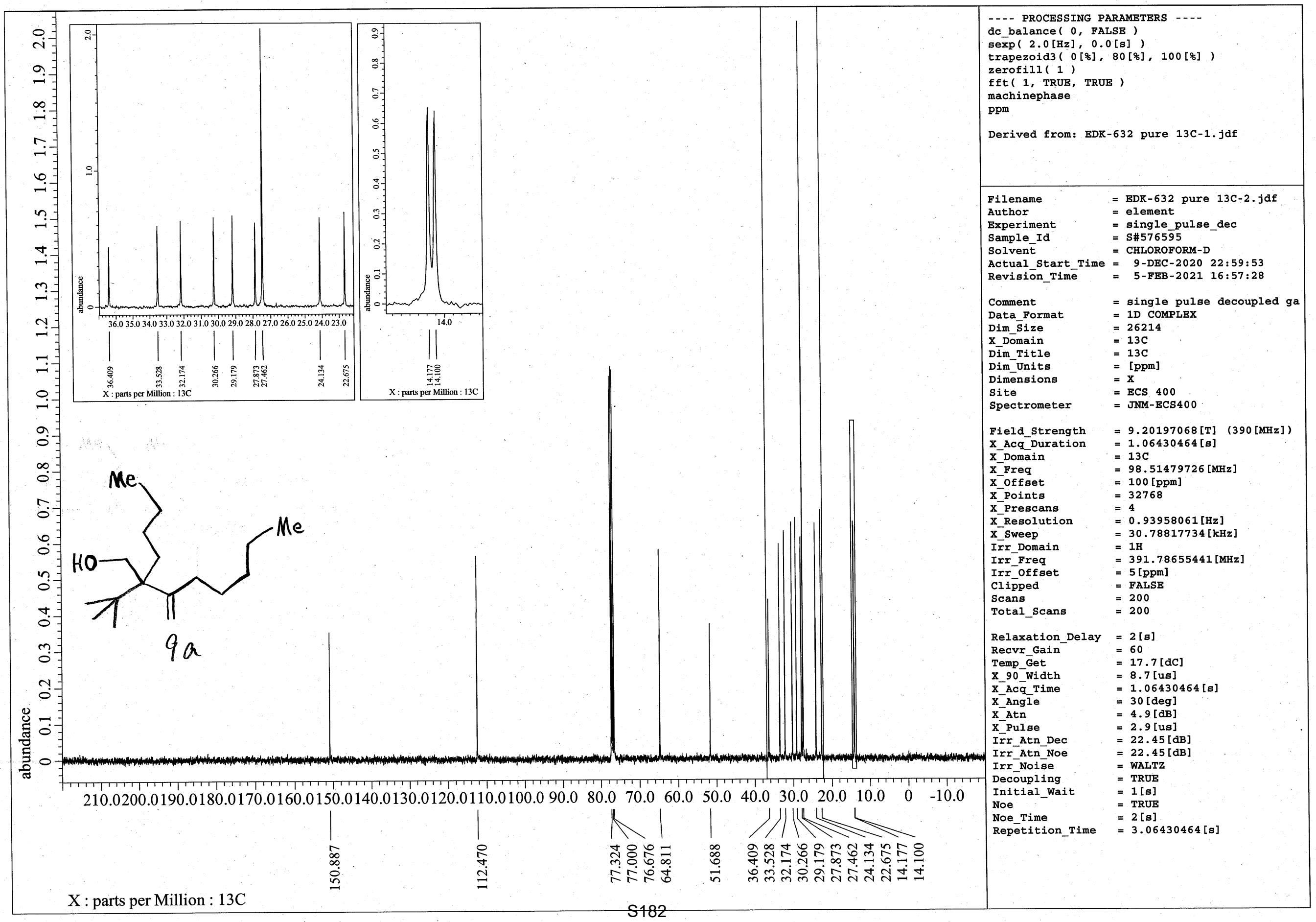




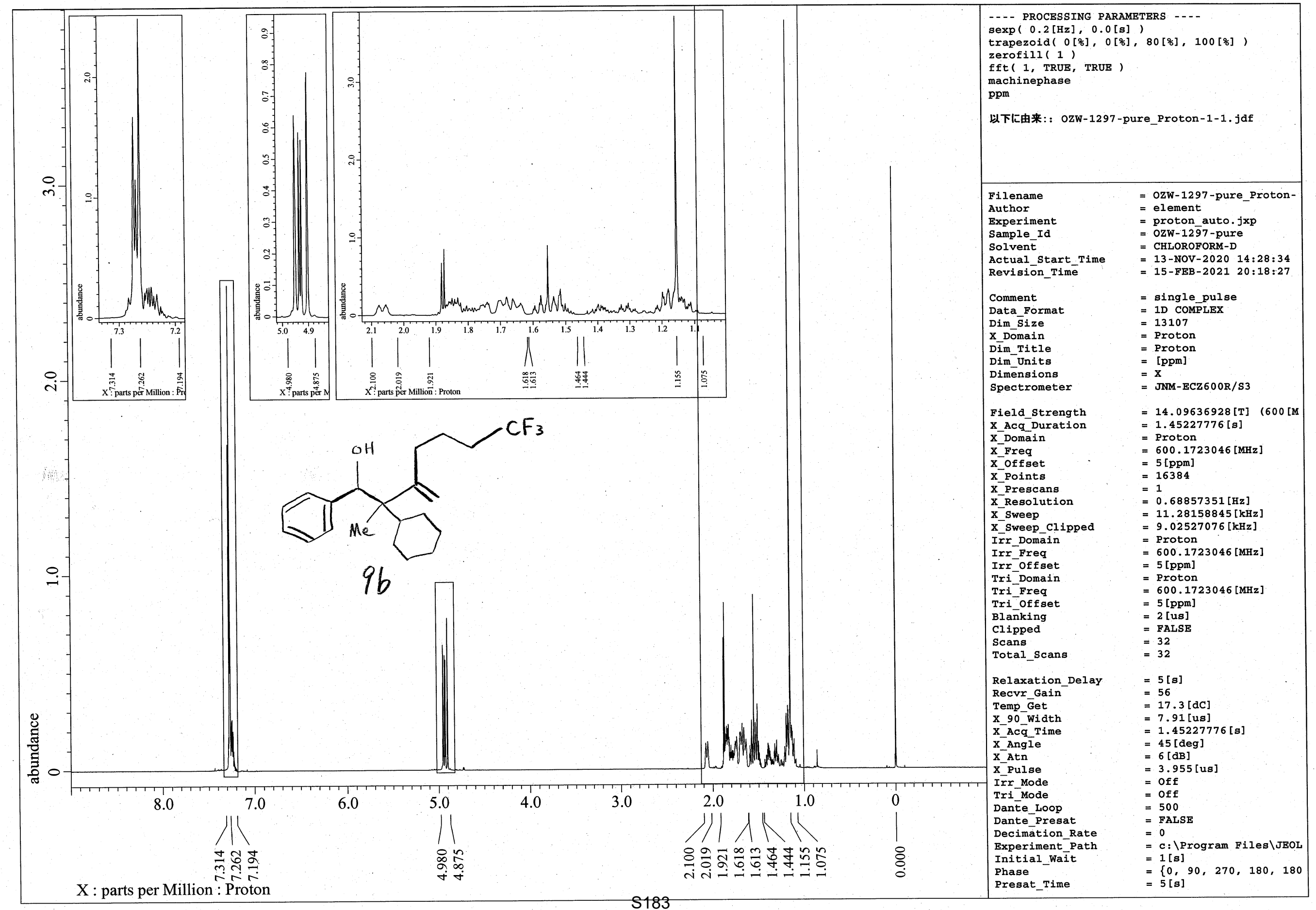




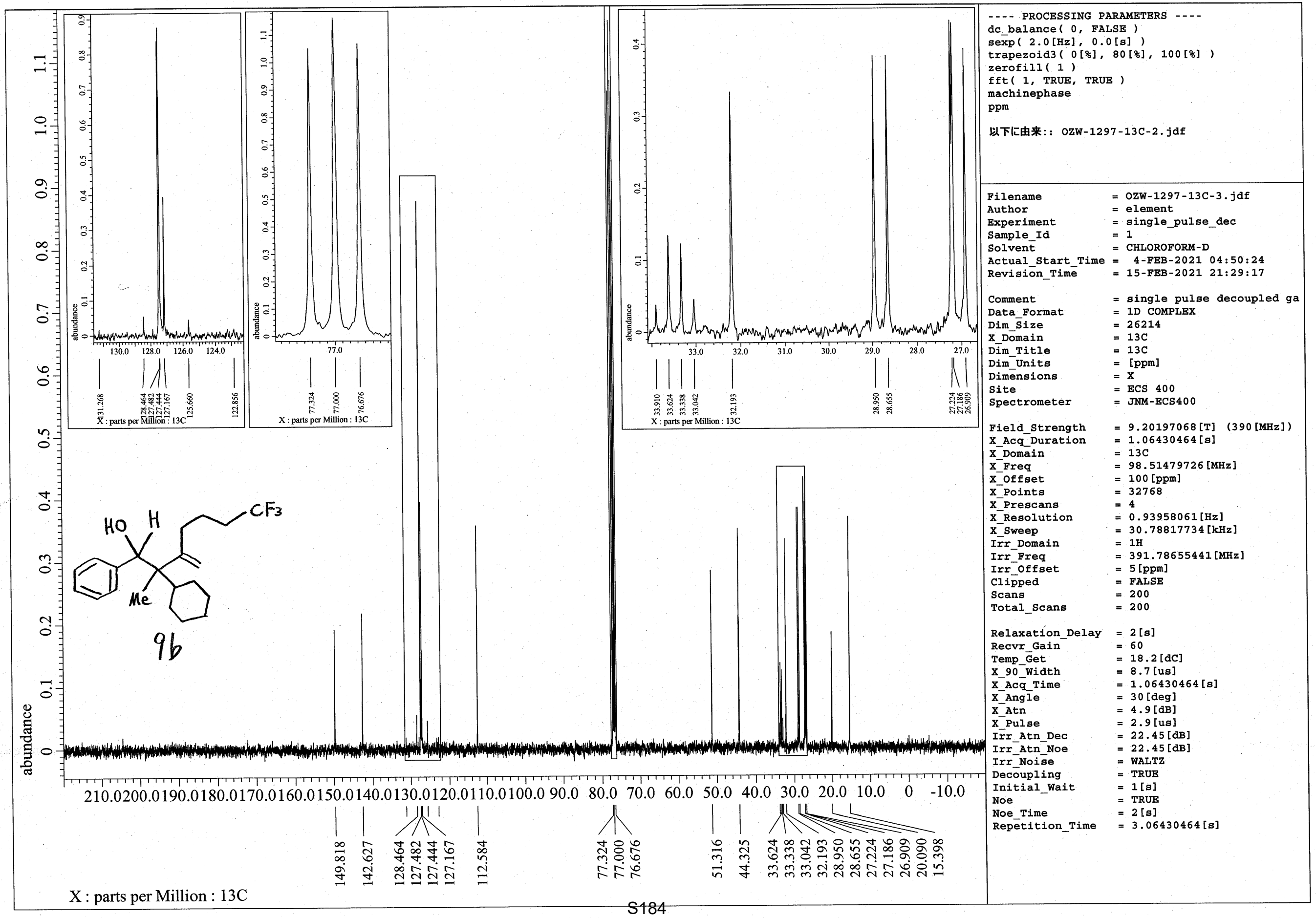




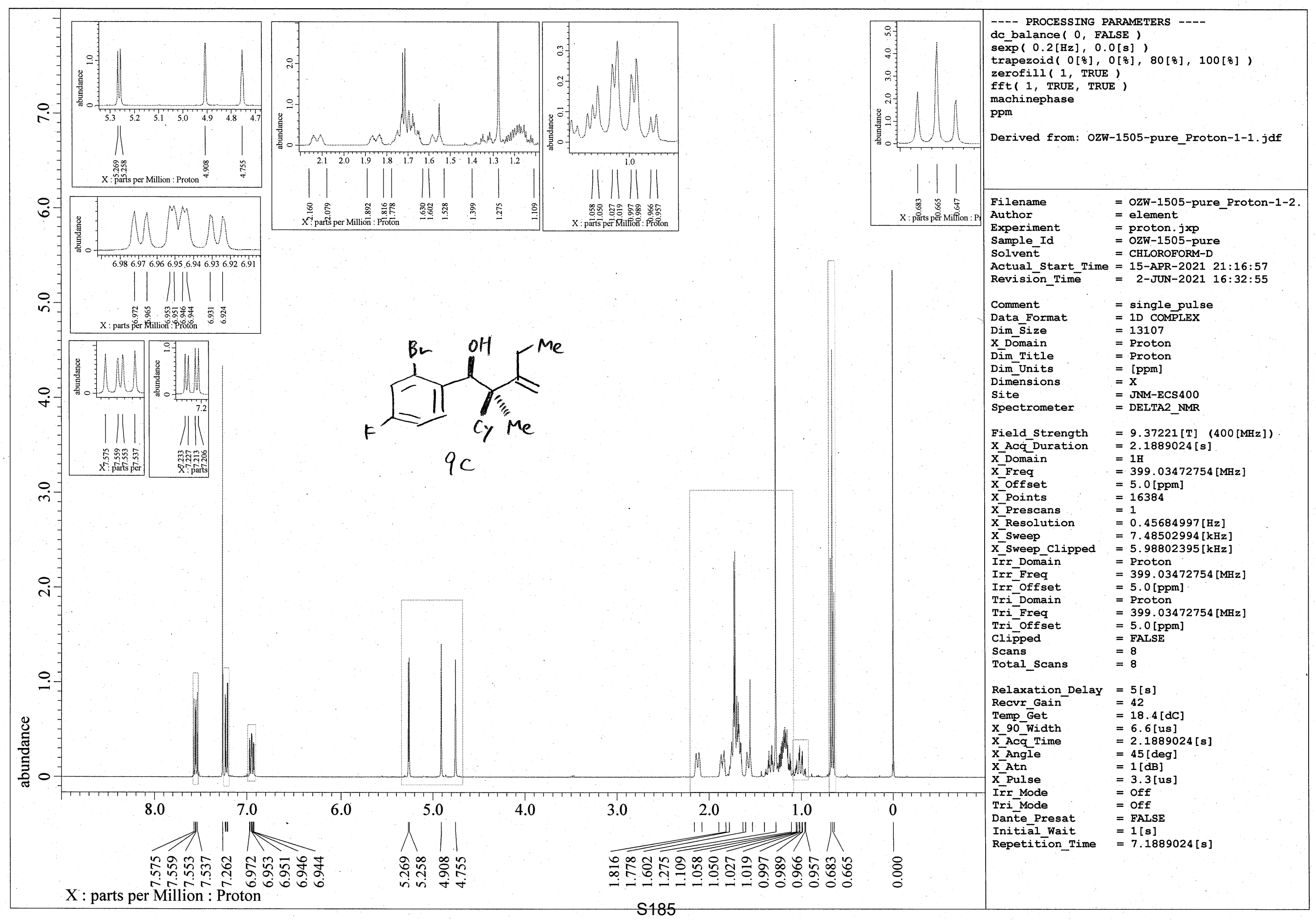




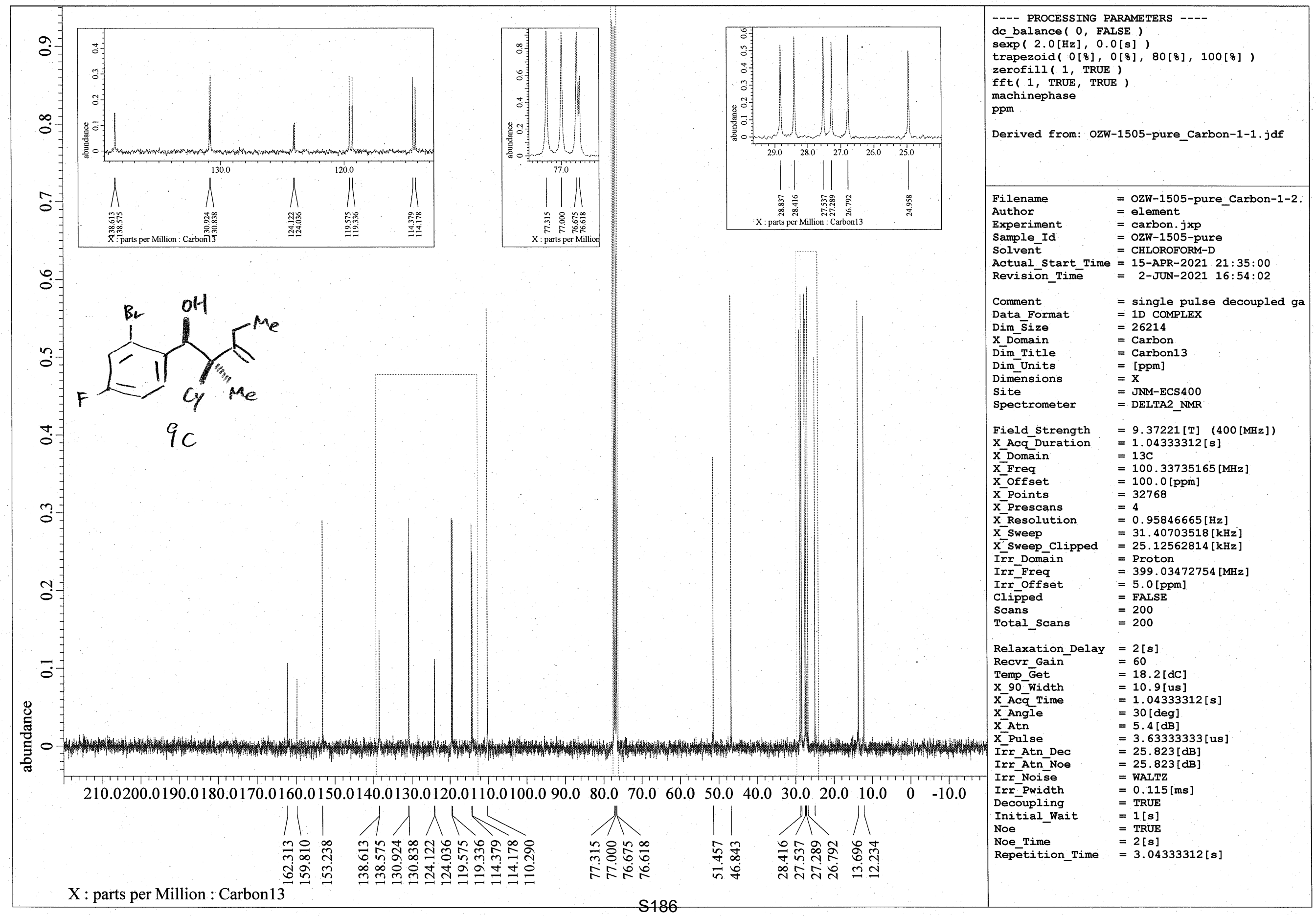




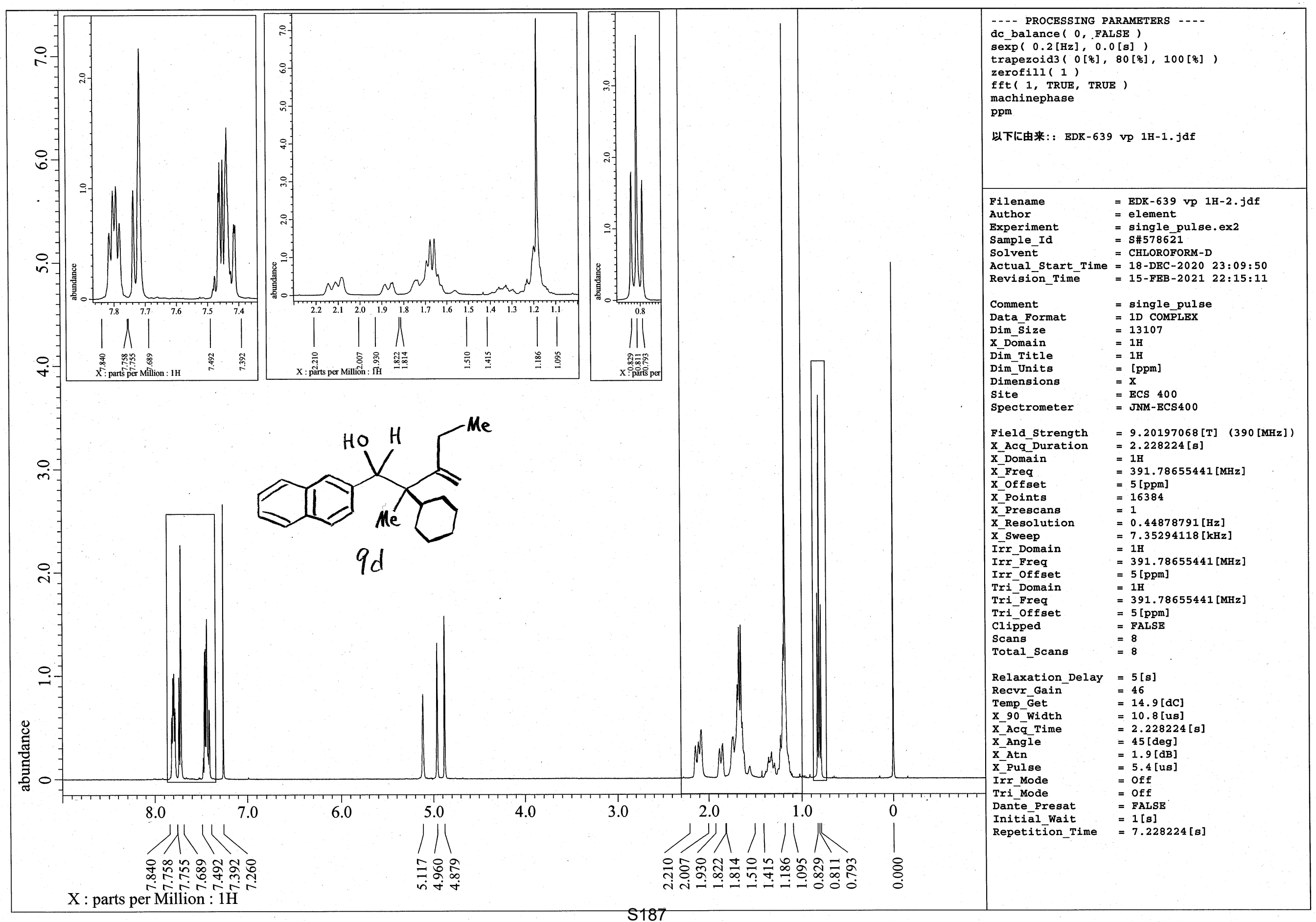




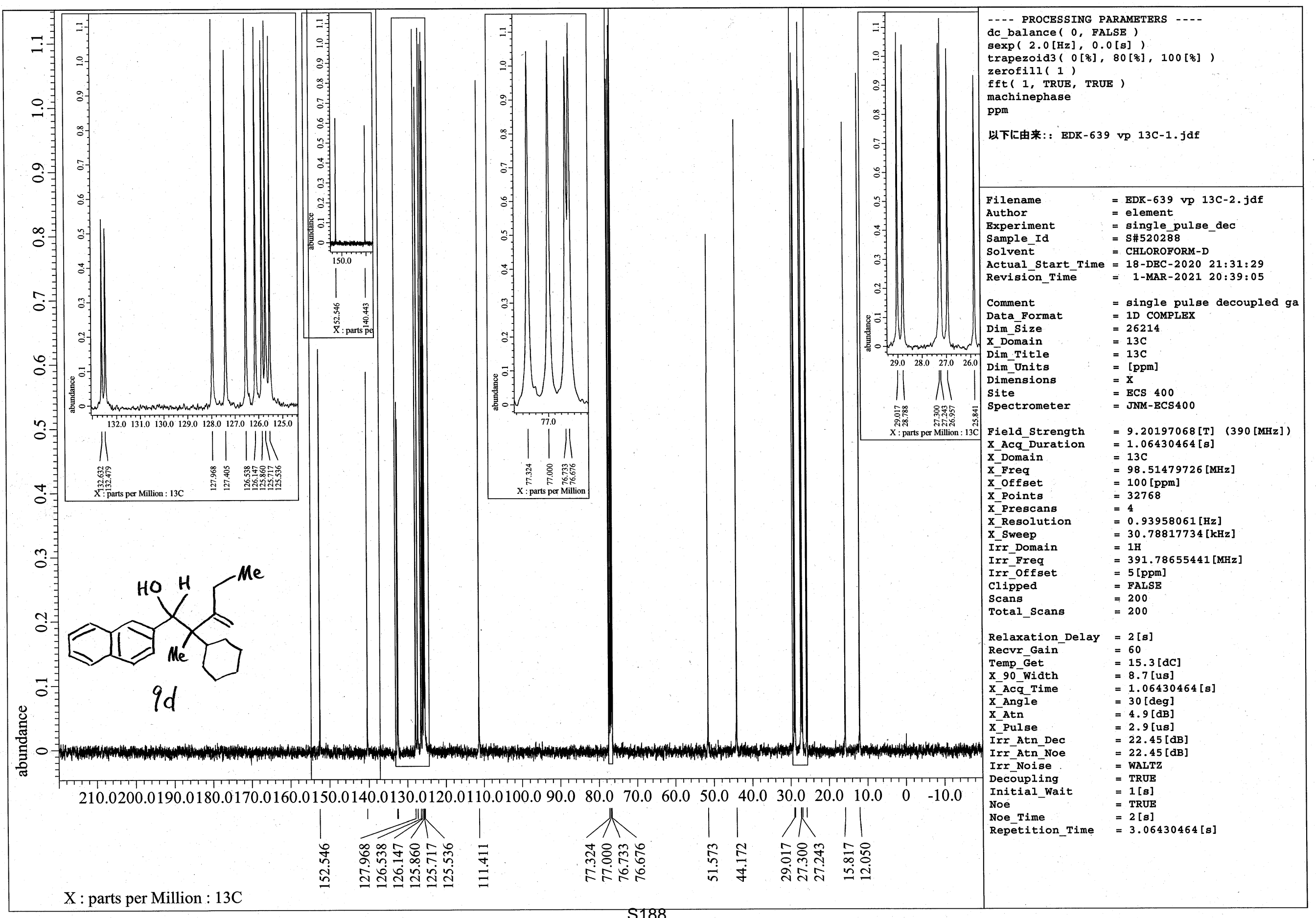




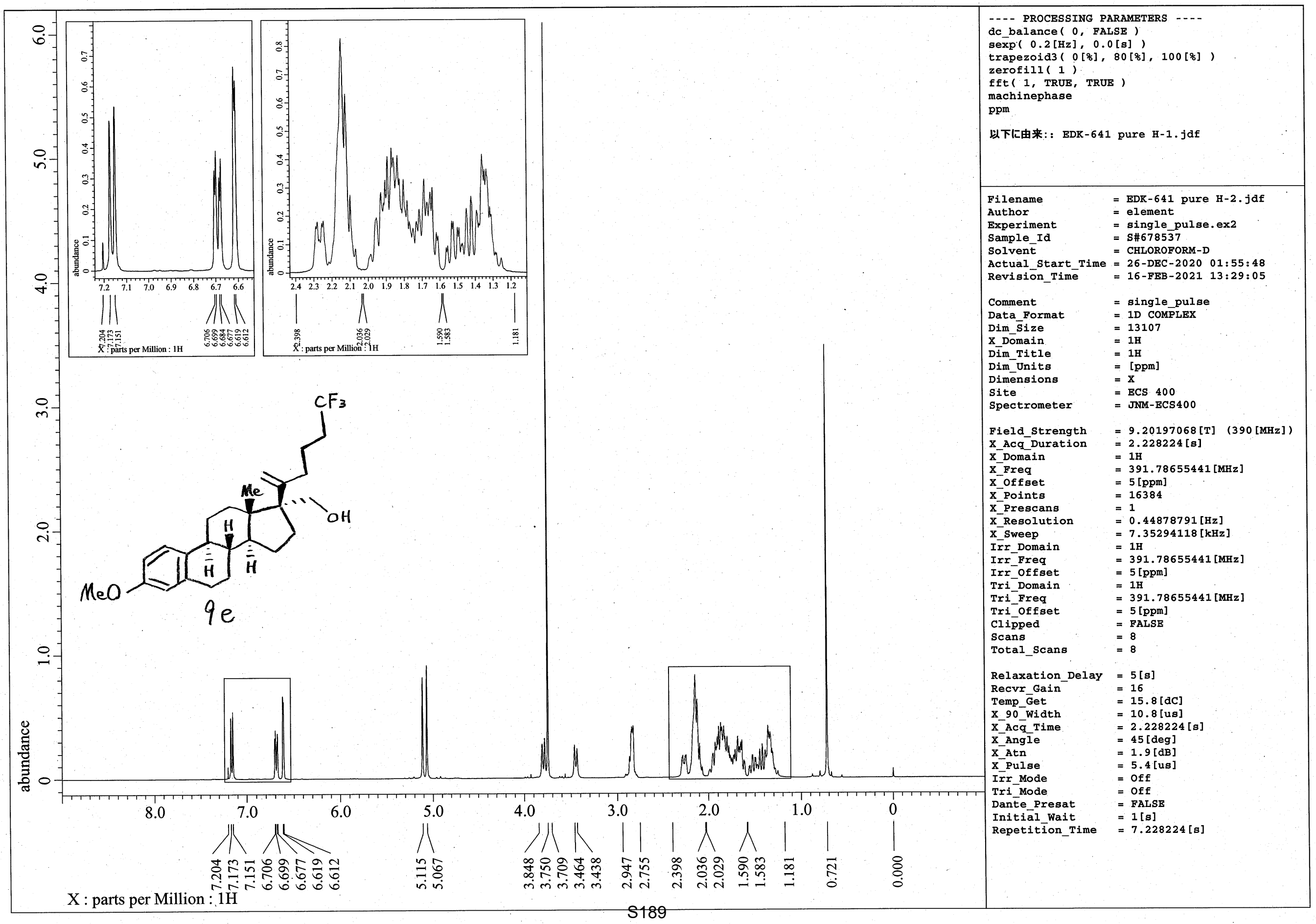




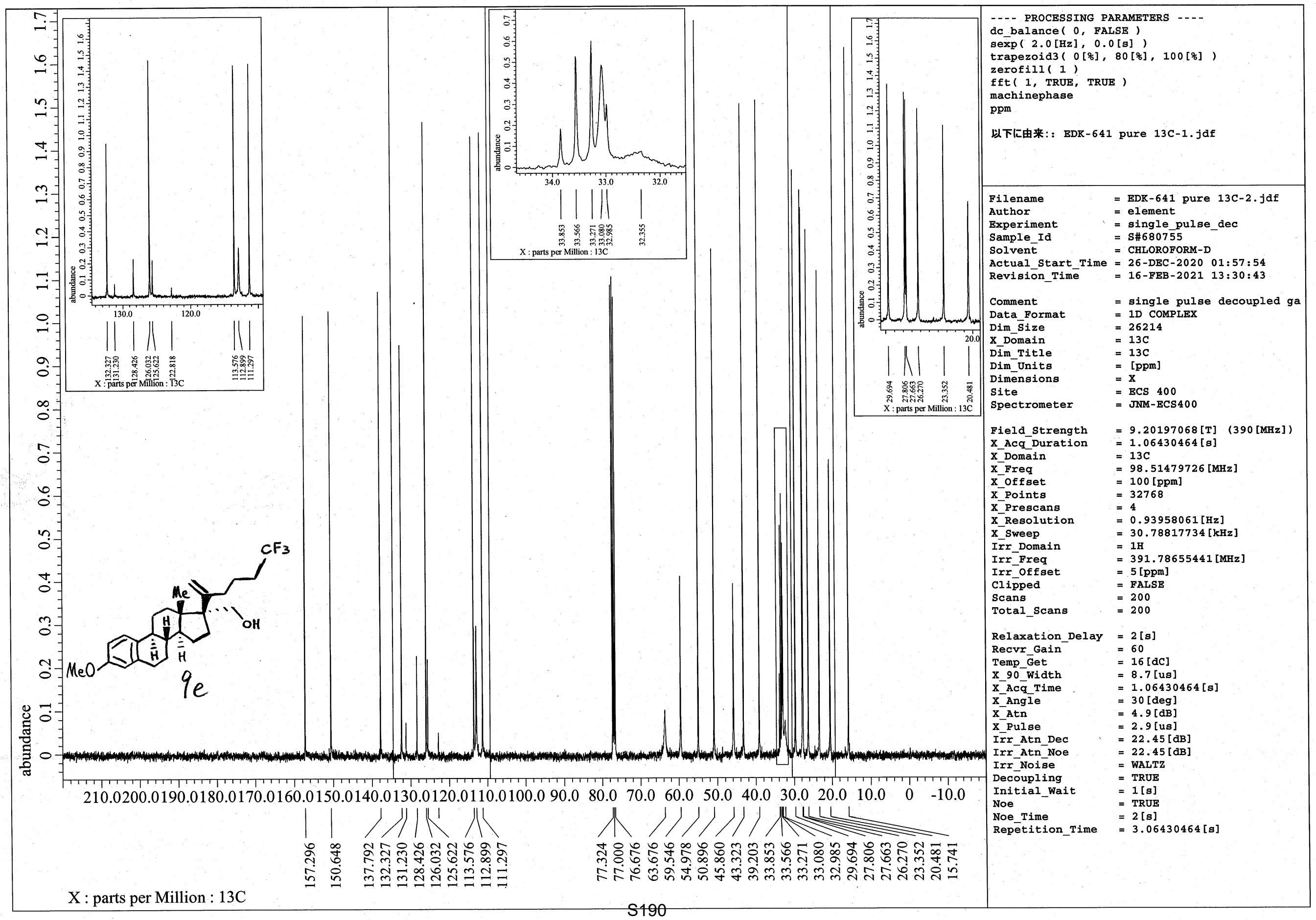

